\title{
The Role of CCR5 in vaccinia virus pathogenesis
}

by

\author{
Ramtin Rahbar
}

A thesis submitted in conformity with the requirements for the

degree of Doctor of Philosophy

Graduate Department of Immunology

University of Toronto

C Copyright by Ramtin Rahbar, 2009 


\title{
The Role of CCR5 in vaccinia virus pathogenesis
}

\author{
Degree of Doctor of Philosophy, 2009
}

\author{
Ramtin Rahbar
}

Graduate Department of Immunology

\section{University of Toronto}

Viral appropriation of chemokine receptors is an effective way to prevent a host immune response against the invading virus. Many viruses, including poxviruses, subvert the host immune response by encoding several chemokine receptor homologues, capable of binding to and thereby precluding chemokines from activating their cognate cell surface receptors. All poxviruses employ strategies to modulate chemokine activity, including virus-encoded chemokine-binding proteins, receptor homologues and ligand mimics. The potential for the involvement of certain chemokine receptors in poxviral infection was suggested in studies utilizing the rabbit poxvirus, myxoma. Specifically, CCR5 was implicated in mediating cell target susceptibility to infection. Our data suggest virus-CCR5 interactions may lead to the selective activation of distinct signaling pathways that are advantageous for the virus.

VACV, a member of the poxvirus family, produces two structurally distinct forms of virions, the intracellular mature virus (IMV) and the extracellular enveloped virus (EEV), for which the immediate events following cell entry are ill- 
defined. Using confocal microscopy, we provided evidence that IMV and EEV enter both permissive and non-permissive cells, and that introduction of CCR5 into non-permissive cells - mouse fibroblasts and human PM1 T cells - renders them permissive for VACV replication. We showed that virus activation of CCR5 leads to the selective activation of distinct signaling pathways that are advantageous for the virus. We demonstrated that VACV infection in permissive cells is inhibited by siRNA knockdown of cell surface CCR5 expression and by the CCR5 antagonist, TAK-779. The importance of tyrosine phosphorylation of CCR5 was suggested by the observation that introduction of a CCR5 mutant, in which all the intracellular tyrosines are replaced by phenylalanines, effectively reduces VACV infection in permissive cells. Moreover, tyrosine-339 was implicated in CCR5 as the critical residue for mediating viral infection, since cells expressing CCR5.Y339F do not support viral replication. The cascade of events that leads to permissive phenotype of these cells includes phosphorylation activation of multiple signaling effectors: Jak-2, IRS-2, ERK1/ 2 and Grb2. These data were supported by findings that viral replication in permissive CCR5 expressing cells is blocked by Herbimycin A, and the Jak2 inhibitor, tyrophostin AG490, but not pertussis toxin. Viewed altogether, a critical role of post-entry events, specifically intracellular tyrosine phosphorylation events, was established in determining permissiveness of cells to VACV replication. Furthermore, evidence was provided that introduction of CCR5 in primary human $\mathrm{T}$ cells renders them permissive to VACV replication. Since permissive infection of T cells might represent a mechanism for VACV dissemination throughout the lymphatic system, we hypothesized that the absence of CCR5 may be protective against VACV infection in vivo. 
To test this hypothesis, wild-type and CCR5 null mice were challenged with VACV by intranasal inoculation. In time course studies we identified aggressive viral replication in the lungs and spleens of $\mathrm{CCR}^{+/+}$mice, with no evidence of infection in the $\mathrm{CCR}^{-/-}$mice. Moreover, associated with VACV infection, we provided evidence for $\mathrm{CD}^{+}$and $\mathrm{CD}^{+} \mathrm{T}$ as well as $\mathrm{CD} 11 \mathrm{c}^{+}$and $\mathrm{F} 4 / 80^{+}$cell infiltration into the lungs of $\mathrm{CCR}^{+/+}$but not $\mathrm{CCR}^{-/-}$mice, and showed that CCR5-expressing $\mathrm{T}$ cells harbor replicating virus. We showed that this CCR5-dependence is VACV-specific, since CCR5${ }^{1-}$ mice were as susceptible to intranasal influenza (A/WSN/33) infection as CCR5 ${ }^{+/+}$ mice. In a final series of experiments we provided evidence that adoptive transfer of $\mathrm{CCR}^{+/+}$bone marrow into $\mathrm{CCR} 5^{-/}$mice restored VACV permissiveness, with evidence of lung and spleen infection. Taken together, our data showed a critical and novel role for CCR5 in VACV infection and dissemination in vivo.

Moreover, our confocal studies suggested a possible physical interaction between cellular proteins and the VACV in cytosole. Using mass spectrometry-based proteomics, glomulin was identified as a host cell protein that interacts with VACV. Knockdown of glomulin expression in human PM1.CCR5 T cells reduced VACV infection. We demonstrate that treatment of PM1.CCR5 T cells with a c-Met phosphorylation inhibitor led to a significant reduction in VACV infectivity. The data indicated that inhibition of cMet phosphorylation, reduces the cytosolic availability of activated glomulin, thus leading to a decrease in VACV infectivity. These data identify glomulin as a permissivity factor for VACV infection, and as a potential therapeutic target for VACV. 


\section{ACKNOWLEDGEMENTS}

This thesis arose from my research that was done in Dr. Eleanor Fish's research group. In that time, I have worked with a great number of people who have contributed in many ways to my research and thesis that I feel deserve special mention. It is a pleasure to convey my gratitude to them all in my humble acknowledgment. First and foremost I offer my sincerest gratitude to my supervisor Dr. Eleanor Fish, who has supported me throughout my thesis with her patience and knowledge while providing me the room to work in my own way. Above all and the most needed, she provided me encouragement in various ways. Her scientific intuition provided me with a constant oasis of ideas and passion towards research, which exceptionally inspired and enriched my growth as a student, researcher and scientist. I am indebted to her more than she knows.

I gratefully acknowledge my committee members Dr. Kevin Kain and Dr. Michele Anderson for their advice, and crucial contribution.

I was extraordinarily fortunate to work with many wonderful individuals over the years in the Fish lab, who not only taught me a lot, but have always been a constant source of equanimity and friendship. Beata, the role model for hard workers in the lab, I would like to thank you for being the one who taught me a lot. I am proud to say that I had the opportunity to work with an exceptionally experienced scientist like you. You are the true meaning of patience, kindness and friendship. Thank you Jianbing, Celeste, Sham and Milly for sharing your love and experiences with me. Thomas, you were/are like my brother. You gave me hope and courage when I felt most restless and worried. 
Your sport wit and charismatic personality have always provided me with clearer picture of what to expect from life. You were a good companion during some otherwise exhausting and disappointing experiments, and although you did once or twice explain the rules to baseball I must confess to have forgotten every word since. Thank you for everything and I am proud to say that I found a lifetime friend. Carole, thank you for your valuable advice in our scientific/non-scientific discussion and for reading my long manuscripts. I am grateful in every possible way and hope to keep up our collaboration in the future. I wish you, Kip and Kaycee a lifetime of joy and happiness. Danlin, my younger brother; thank you very much for all your positive attitude and your humble hand of help anytime I asked. I am sure one day you will get what you deserve of this world. You have to promise to keep your passion alive. Daniel, thank you for your invaluable scientific discussion, help, friendship and encouragement. Your trustworthy personality and honest mannerism truly make you the amazing friend that everyone wishes for. Jay, thank you for letting me steal your pipette tips. I wish you and your family a future full of joy and success. Erin, thank you for being such a good and experienced ear whenever and wherever. I learned a lot from you. Your smart and always-working way of thinking give you a unique personality I always looked to. Thank you for introducing Jason Mraz to me. Olivia, I wish you a very successful study period. Thank you for your charming wit that you brought to our group. Joanne, I wish you a very successful time in Montreal.

Thank you to the administrative assistants of the Department of Immunology, Rejeanne Puran, Lynne Omoto and Sherry Kuhn, for their help. 
I wish to express my appreciation to my family, especially to my parents, whose enormous love and support give me courage and strength to continue. Special thanks to Juliet for being such an amazing support, a sincere ear and a wonderful source of encouragement for me during all these years.

This work could have not been accomplished without the dedication of my mice that contributed greatly to my success.

I would also like to convey thanks to the Canadian Institute of Health Research (CIHR) for providing me financial support to perform this research. 


\section{TABLE OF CONTENTS}

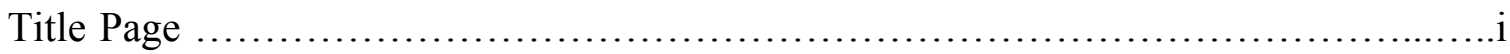

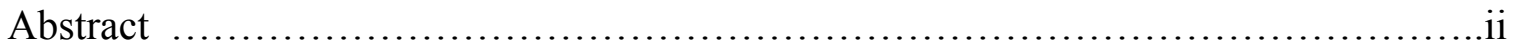

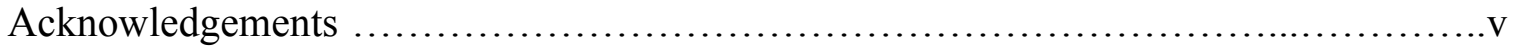

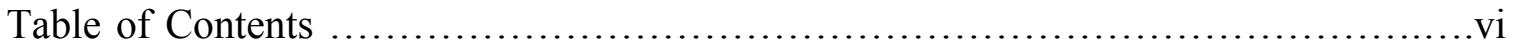

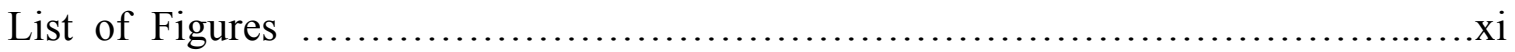

List of Tables ........................................................................iii

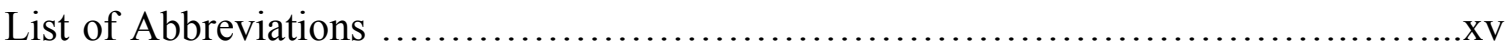

CHAPTER 1: Introduction .................................................

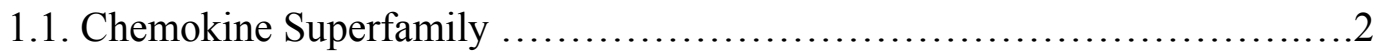

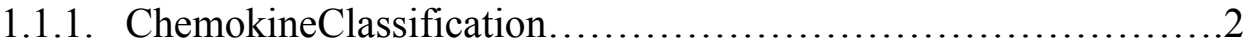

1.2. Chemokine Receptors ................................................

1.3. Chemokine-chemokine receptor binding and signal transduction .............14

1.3.1. Chemokine-glycosaminoglycan (GAG) binding .................17

1.3.2. Chemokine mediated signal transduction.......................19

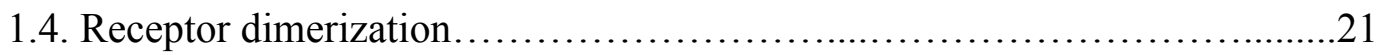

1.5. Receptor desensitization and endocytosis..............................22

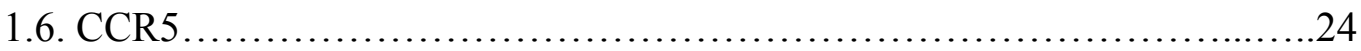

1.6.1. CCR5 mediated signalling ...................................... 31

1.6.2. CCR5: a potential therapeutic target............................. 36

1.7. Viral subversion of host chemokine/chemokine receptor system.............38

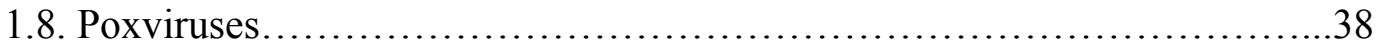




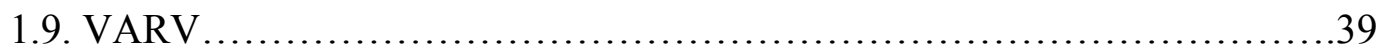

1.9.1. Smallpox Pathogenesis and Symptoms..........................40

1.9.2. Vaccination.................................................... 41

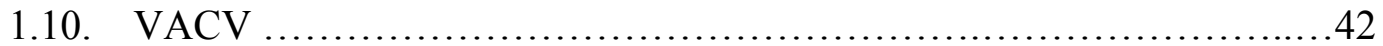

1.10.1. VACV Replication cycle.......................................43

1.10.2. VACV entry into the host cell................................50

1.10.3. WASp: A potential effector of vaccinia virus infection............57

1.10.4. VACV pathophysiology.......................................64

1.10.5. VACV-induced immunomodulation..............................66

1.10.5.1. Virokines and Viroceptors.......................66

1.10.5.1.1. Proteins that block the complement pathways. 68

1.10.5.1.2. Proteins that block antigen presentation to the MHC..........................................69

1.10.5.1.3. Proteins that block interferon signaling pathways...................................71 1.10.5.1.3.1 Intracellular inhibitors of IFN.....71

1.10.5.1.3.2. Extracellular inhibitors of IFN....72

1.10.5.1.4. A soluble IFN- $\gamma$ receptor......................73

1.10.5.1.5. Interferon- $\alpha / \beta$ binding proteins...............73

1.10.5.1.6. Proteins that inhibit IFN- $\gamma$ induction by binding to IL-18 .................................74

1.10.5.1.7. Soluble proteins that inhibit tumor necrosis factor (vTNFr) ............................75

1.10.5.1.8. Intra- and extra-cellular Inhibition of IL-1 ....77

1.10.5.1.9. Modulation of Chemokines....................77

1.10.5.1.10. Poxviral homologs of other host proteins..79

1.10.5.2. Poxviral inhibition of apoptosis...................79

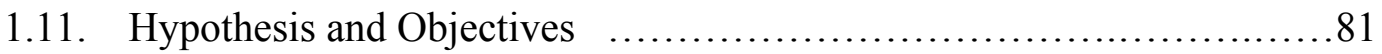

\section{CHAPTER 2: Vaccinia virus activation of CCR5 invokes tyrosine phosphorylation signaling events that support virus replication ............................... $81-137$}

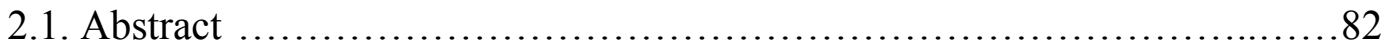

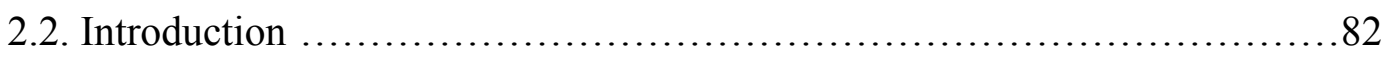

2.3. Results

2.3.1. Ectopic expression of CCR5 confers a permissive phenotype for

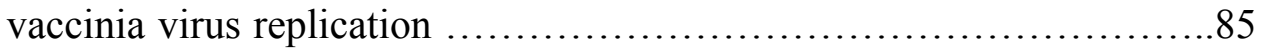


2.3.2. Absence of VACV late gene transcription in non-permissive cells. .98

2.3.3. A CCR5 antagonist inhibits vaccinia virus infection .98

2.3.4. Vaccinia virus induces tyrosine phosphorylation of cellular intermediates 101

2.3.5. Vaccini virus induces tyrosine phosphorylation of signalling effectors in permissive cells. 105

2.3.6. Vaccinia virus co-localizes with CCR5 in permissive cells. 110

2.4.Discussion

2.5.Materials and Methods

2.5.1. Cells and virus

2.5.2. Measurements of viral infectivity

2.5.3. Analysis of CCR5 surface expression by flow cytometry ...128

2.5.4. Cell lysis and immunoblotting ..............................128

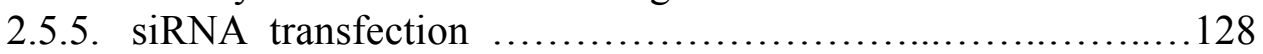

2.5.6. CCR5 mutagenesis ...............................129

2.5.7. Introduction of CCR5, CCR5.Y307F, CCR5.Y339F and CCR5.Y $\Delta 3 \mathrm{~F}$ into cells .................................130

2.5.8. Immunohistochemical analysis and confocal microscopy ......130

2.5.9. Reverse Transcription PCR (RT-PCR). 131

CHAPTER 3: A role for CCR5 in dissemination of vaccinia virus in vivo...138-192

3.1. Abstract

3.2. Introduction 140

\subsection{Results}

3.3.1. $\mathrm{CCR}^{-/-}$mice are less susceptible to systemic VACV infect. 142

3.3.2. VACV infection of $\mathrm{CCR} 5^{+/ t}$ but not $\mathrm{CCR} 5^{-/-}$mice leads to infiltration of $\mathrm{CD} 4+$ and $\mathrm{CD} 8+\mathrm{T}$ cells into the lungs

3.3.3. Expression of CCR5 influences the $\mathrm{T}$ cell population of secondary lymphoid organs 151

3.3.4. TAK-779 reduces VACV dissemination 152 
3.3.5. Synchronous effects of VACV infection on antigen presenting cells in $\mathrm{CCR}^{+/+}$and $\mathrm{CCR}^{-/-}$mice

3.3.6. CCR5 expression does not contribute to influenza A/WSN/33 infection.

3.3.7. Adoptive transfer of $\mathrm{CCR}^{+/+}$leukocytes into $\mathrm{CCR} 5^{-/}$mice restores susceptibility to VACV infection .................................164

3.4. Discussion 167

3.5. Materials and Methods

3.5.1 Animals ................................................... 172

3.5.2. Viruses ..................................................... 173

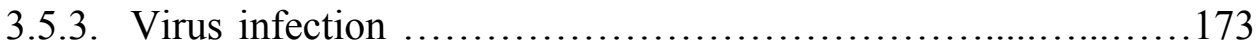

3.5.4. Tissue isolation and viral titration ............................174

3.5.5. Leukocyte isolation and Flow cytometry .......................174

3.5.6. Histology and Immunohistochemistry .......................175

3.5.7. Bone marrow transplantation..............................176

3.5.8. Administration of TAK-779, a CCR5 antagonist...............177

3.5.9. Statistical Analysis ........................................177

CHAPTER 4: Glomulin, a protein involved in VACV infection. $193-218$

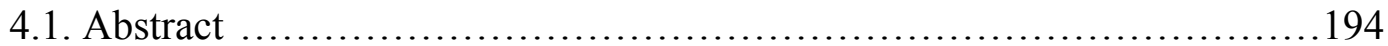

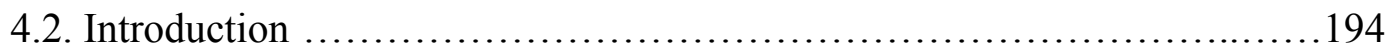

4.3. Results

4.3.1. A4L-EGFP-VACV interacts with multiple host proteins .........196

4.3.2. Knockdown of glomulin reduces VACV infection ..............197

4.3.3. Inhibition of cMET-mediated phosphorylation reduces VACV

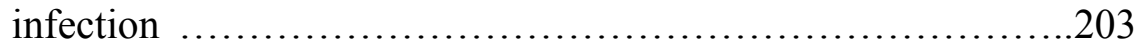

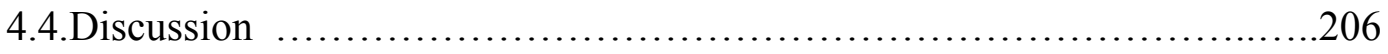

4.5. Materials and Methods

4.5.1. Cell lines and viruses ..........................................................209

4.5.2. In-solution protein digestion .............................................210

4.5.3. Multidimensional protein identification technology (MudPIT)

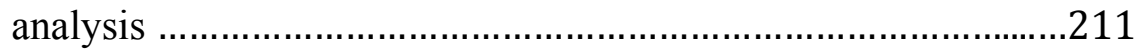

4.5.4. Protein identification, validation and grouping .........................212

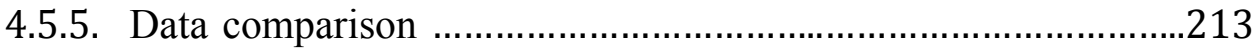

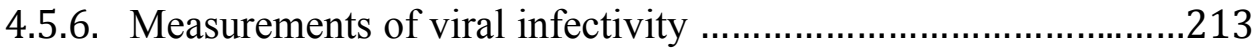

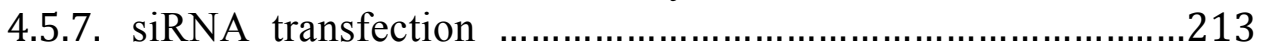

4.5.8. Reverse-transcription PCR ......................................214 


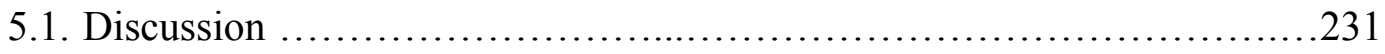

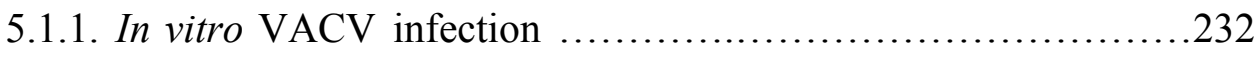

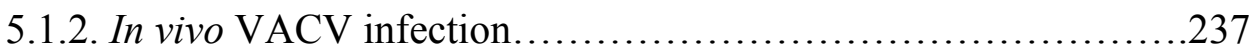

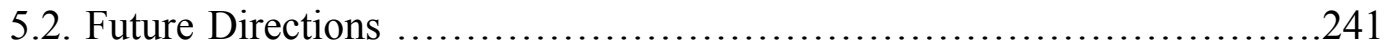

5.2.1. Characterization the role of WASp in VACV infection..........241

5.2.2. VACV regulation of host cell gene expression..................242

5.2.3. Examination of the antiviral effects of IFNs..................242

5.2.4. Characterization of specific VACV factors that bind to intracellular targets...................................................243

CHAPTER 6: Dissemination of Work Arising from this Thesis .................236

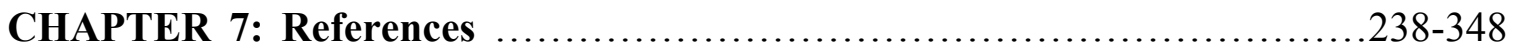




\section{LIST OF FIGURES}

\section{CHAPTER 1}

Figure 1.1. Chemokine receptors and their role in diseases ...................... 16

Figure 1.2. Schematic representation of CCR5 structure $\ldots \ldots \ldots \ldots \ldots \ldots \ldots \ldots . \ldots 30$

Figure 1.3. Schematic representation of CCL5-CCR5 signaling event.............35

Figure 1.4. Schematic overview of VACV life cycle .........................49

Figure 1.5. Schematic representation of IMV and EEV entry $\ldots \ldots \ldots \ldots \ldots \ldots \ldots 5$

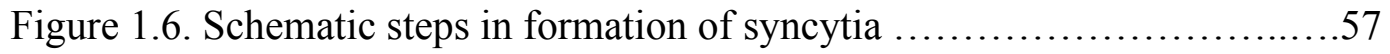

Figure 1.7. Schematic representation of VACV-CCR5 signaling events.........61

Figure 1.8. Schematic representation of WASp structure and signaling pathway.63

\section{CHAPTER 2}

Figure 2.1. Knockdown of CCR5 in NIH3T3.CD4.CCR5 cells reduces vaccinia v virus infectioin...............................................88

Figure 2.2. Introduction of CCR5 into NIH3T3.CD4.neo cells renders them perm permissive for vaccinia virus infection........................90

Figure 2.3. Introduction of CCR5 but not CCR5.Y339 into primary human T-cells renders them permissive for vaccinia virus infection..............94

Figure 2.4. Introduction of CCR5 but not CCR5.Y339 or CCR5.YA3F into PM1 $\mathrm{T}$-cell line renders them permissive for vaccinia virus infection ...96

Figure 2.5. Replication of VACV is restricted in PM1 cells expressing CCR5.Y339F but not CCR5 ..................................99

Figure 2.6. Absence of VACV late gene transcription fails in PM 1.CCR5Y339F cells 
Figure 2.7. Pharmacological inhibition of vaccinia virus infection in PM1.CCR5cells.

Figure 2.8. Vaccinia virus induces tyrosine phosphorylation of IRS-2, Grb2 and Erk $1 / 2$ in permissive but not in non-permissive cells

Figure 2.9. CCR5 and IMV vaccinia virus co-localize in permissive PM1.CCR5 T cells

Figure 2.10. CCR5 internalizes in response to vaccinia virus in PM1.CCR5 but not PM1.CCR5Y339F cells.

Supplementary Figure 2.1. Replication of EEV is blocked in the cells expressing

CCR5.Y339F but not CCR5.

Supplementary Figure 2.2. EEV replication is restricted in the cells expressing

CCR5.Y339F but not CCR5

\section{CHAPTER 3}

Figure 3.1. $\mathrm{CCR}^{-/-}$mice are less susceptible to VACV infection 146

Figure 3.2. Intranasal inoculation with VACV leads to an increase in BAL viral titer and influx of $\mathrm{CD}^{+}$and $\mathrm{CD}^{+} \mathrm{T}$ cells into the lungs of $\mathrm{CCR}^{+/+}$but not $\mathrm{CCR}^{-/-}$mice

Figure 3.2. VACV infection of $\mathrm{CCR}^{+/+}$but not $\mathrm{CCR}^{-/-}$mice influences the $\mathrm{T}$ cell responses in secondary lymphoid organs

Figure 3.4. TAK-779 reduces splenic VACV infection 156

Figure 3.5. VACV infection modulates CD11c+ dendritic cell and F4/80+ macrophage populations in the mLNs and spleens of $\mathrm{CCR}^{+/+}$but not $\mathrm{CCR}^{-/-}$mice

Figure 3.6. $\mathrm{CCR}^{-/-}$mice are permissive for influenza A/WSN/33 infection ...163

Figure 3.7. Adoptive transfer of $\mathrm{CCR}^{+/+}$leukocytes into $\mathrm{CCR} 5^{-/-}$mice restores permissiveness to VACV infection

Supplementary Figure 3.1. Intranasal infection with VACV WR or EGFP- VACV induces similar immune responses 
Supplementary Figure 3.2. Intranasal inoculation with VACV leads to an influx of $\mathrm{CD} 4+$ and $\mathrm{CD} 8+\mathrm{T}$ cells into the lungs of $\mathrm{CCR}^{+/ t}$ but not $\mathrm{CCR}^{-/-}$ mice 183

Supplementary Figure 3.3. Intranasal infection with VACV results in activation of $\mathrm{CD} 4+$ and $\mathrm{CD} 8+\mathrm{T}$ cells in $\mathrm{mLN}$ of $\mathrm{CCR}^{+/+}$but not $\mathrm{CCR} 5^{-/}$mice..186

Supplementary Figure 3.4. Intranasal infection with $10^{5}$ p.f.u. of VACV results in immunological response in $\mathrm{CCR}^{+/+}$and to a lesser extent in $\mathrm{CCR} 5^{-/-}$ mice 188

Supplementary Figure 3.5. $\mathrm{CCR}^{-/-}$mice are permissive for influenza A/WSN/33

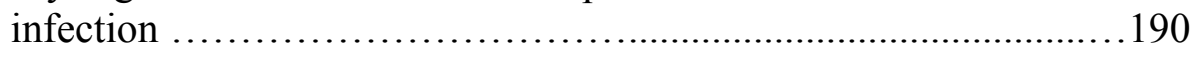

Supplementary Figure 3.6. $\mathrm{CCR}^{-/-}$mice are permissive for influenza A/WSN/33 infection ..............................................................192

\section{CHAPTER 4}

Figure 4.1. MudPIT identifies host proteins that physically associate with A4L-

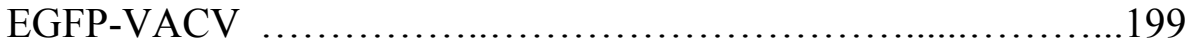

Figure 4.2. Knockdown of glomulin reduces VACV infection.................202

Figure 4.3. Inhibition of c-MET activation reduces VACV infection............205 


\section{LIST OF TABLES}

\section{CHAPTER 1}

Table 1.1. The chemokine superfamily, nomenclature and common names for

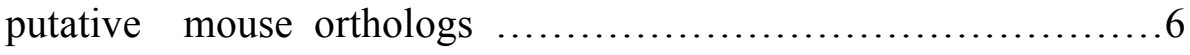

Table 1.2. Chemokine receptors and their ligands...........................11

\section{CHAPTER 4}

Table 4.1. Empirical evaluation of false recovery rate ......................200

Supplementary Table 4.1. Gene Ontology (GO) representing the biological processes of proteins in the CCR5 specific cluster that interact with VACV ...................................................216

Supplementary Table 4.2. GO representing the molecular function of proteins in

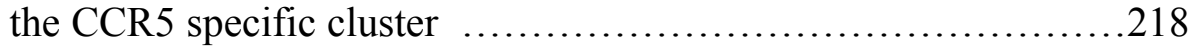




\section{LIST OF ABBREVIATIONS}

$\mathrm{Ab}$

ADP

AIDS

AICD

AOP-CCL5

APC

$\operatorname{Arp} 2 / 3$

Bcl-2

bp

BRET

CCL5

CCR5

CCX-CKR

$\operatorname{Cdc} 42$

CEV

c-Myc

CPN

CPXV

CRD

CS

C-terminus

CTL

CXCL

CXCR
Antibody

Adenosine diphosphate

Acquired immunodeficiency syndrome

Activation induced cell death

Aminooxypentane-CC chemokine ligand 5

Antigen presenting cell

Actin-related proteins $2 / 3$

B cell lymphoma-2

Base pair

Bioluminescence resonance energy transfer

CC chemokine ligand 5

$\mathrm{CC}$ chemokine receptor 5

ChemoCentryx chemokine receptor

Cell division cycle 42

Cell-associated enveloped virus

Cellular-myelocytomatosis virus oncogene

Vaccinia virus strain Copenhagen

Cowpox virus

Cystein-rich domains

Chondroitin sulphate

Carboxy-terminus

Cytotoxic T lymphocyte

CXC chemokine ligand

CXC chemokine receptor 
$\mathrm{CX}_{3} \mathrm{CL}$

$\mathrm{CX}_{3} \mathrm{CR}$

DAD

DAG

DARC

DC

DNA

DPG

DRY

DS

DTT

ECL

EDTA

EGTA

EGF

EEV

eIF

ELR

EM

ERK

F-actin

FACS

FAK

FasL

FCS

FITC
$\mathrm{CX}_{3} \mathrm{C}$ chemokine ligand

$\mathrm{CX}_{3} \mathrm{C}$ chemokine receptor

Defender against cell death

Diaceylglyerol

Duffy antigen receptor for chemokines

Dendritic cell

Deoxyribonucleic acid

Diphosphoglycerate

Aspartate-Arginine-Tyrosine

Dermatan sulphate

Dithiothreitol

Extra-cellular loop

Ethylenediamine tetra-acetic acid

Ethylene glycol-bis (2-aminoethylether)-N'N'N'N'-tetra-acetic acid

Epidermal growth factor

Extracellular enveloped virions

Eukaryotic translation initiation factor

Glutamate-Leucine-Arginine

Electron microscopy

Extracellular signal-related kinase

Filamentous actin

Flourescence activated cell sorter

Focal adhesion kinase

Fas antigen ligand

Fetal calf serum

Flourescein isothiocynate 
FKBP12

FLF

FRAP/mTOR

FRET

GABA

GAG

GAP

GAPDH

GDP

GEF

GFP

GM-CSF

gp120

GPCR

GRK

GTP

HA

HEK

HEV

HIV

HLA

HRP

HS

HSV

IMV

$\mathrm{IP}_{3}$
FK506-binding protein of $12 \mathrm{kDa}$

Fulminant liver failure

FKBP 12-rapamycin-associated protein/mammalian target of rapamycin

Fluorescence resonance energy transfer

$\gamma$-aminobutyric acid

Glycosaminoglycan

GTPase activating protein

Glyceraldehyde 3-phosphate dehydrogenase

Guanosine diphosphate

Guanidine nucleotide exchange factor

Green fluorescent protein

Granulocyte-macrophage colony-stimulating factor

Glycoprotein of $120 \mathrm{kDa}$

G-protein coupled receptor

G-protein receptor kinase

Guanosine triphosphate

Hyaluronic acid

Human embryonic kidney

High endothelial venule

Human immunodeficiency virus

Human leukocyte antigen

Horseradish peroxidase

Heparin sulphate

Herpes simplex virus

Intracellular mature virions

Inositol 1,4,5-phosphate 


\begin{tabular}{|c|c|}
\hline Jnk & c-Jun N-terminal kinase \\
\hline $\mathrm{kDa}$ & Kilodalton \\
\hline KS & Keratin sulphate \\
\hline KSHV & Karposi's sarcoma-associated herpes virus \\
\hline ICAM & Intracellular adhesion molecule \\
\hline IEV & Intracellular enveloped virus \\
\hline IFN & Interferon \\
\hline IL & Interleukin \\
\hline IP & Immunoprecipitation \\
\hline IRES & Internal ribosomal entry segment \\
\hline Jak & Janus kinase \\
\hline LFA & Lymphocyte function-associated antigen \\
\hline LPS & Lipopolysaccharide \\
\hline MAPK & Mitogen-activated protein kinase \\
\hline MCP & Macrophage chemo-attractant protein \\
\hline MEF & Murine embryonic fibroblast \\
\hline Met-CCL5 & Methionine-CC chemokine ligand 5 \\
\hline Met-tRNA & Methionine-transfer ribonucleic acid \\
\hline MHC & Major histocompatibility complex \\
\hline MIP & Macrophage inflammatory protein \\
\hline MLCK & Myosin light chain kinase \\
\hline$\mu \mathrm{M}$ & Micromolar \\
\hline MMP & Matrix metalloproteinase \\
\hline MPXV & Monkeypox virus \\
\hline mRNA & Messenger RNA \\
\hline mTOR & Mammalian target of rapamycin \\
\hline mTORC1 & Mammalian target of rapamycin complex 1 \\
\hline
\end{tabular}




\begin{tabular}{|c|c|}
\hline MVA & Vaccinia virus strain Ankara \\
\hline NYCBOH & New York City Board of Health \\
\hline $\mathrm{NF}-\kappa \mathrm{B}$ & Nuclear factor-kappa B \\
\hline NK & Natural killer \\
\hline NMR & Nuclear magnetic resonance \\
\hline $\mathrm{nM}$ & Nanomolar \\
\hline NP-40 & Nonidet-40 \\
\hline $\mathrm{N}$-terminus & Amino-terminus \\
\hline OD & Optical density \\
\hline OX-PHOS & Oxidative phosphorylation \\
\hline p38 & $38 \mathrm{kDa}$ stress-activated kinase \\
\hline PA & Phosphatidic acid \\
\hline PARP & Poly ADP ribose polymerase \\
\hline PBS & Phosphate buffered saline \\
\hline PCR & Polymerase chain reaction \\
\hline PDK & Phosphoinositide-dependent kinase \\
\hline $\mathrm{PGE}_{2}$ & Prostaglandin $\mathrm{E}_{2}$ \\
\hline $\mathrm{PH}$ & Pleckstrin homology \\
\hline PHA & Phytohaemagglutinin \\
\hline PHAS & Phosphorylated heat and acid soluble protein \\
\hline PI & Propidium iodide \\
\hline PI-3'K & Phosphatidylinositol 3-kinase \\
\hline PIKK & Phosphoinositide kinase-related kinase \\
\hline $\mathrm{PIP}_{3}$ & Phosphatidylinositol 3,4,5-phosphate \\
\hline PKB & Protein kinase B \\
\hline $\mathrm{PKC}$ & Protein kinase $\mathrm{C}$ \\
\hline PKR & Protein kinase $\mathrm{R}$ \\
\hline
\end{tabular}




\begin{tabular}{|c|c|}
\hline PLC $\beta$ & Phospholipase $\mathrm{C} \beta$ \\
\hline PLD & Phospholipase D \\
\hline PMA & Phorbol-12-miristate-13-acetate \\
\hline PMSF & Phenylmethylsulfonylflouride \\
\hline PRR & Pattern-recognition receptors \\
\hline PTEN & Phosphatase and tensin homolog deleted in chromosome ten \\
\hline $\mathrm{pTx}$ & Pertussis toxin \\
\hline RA & Rheumatoid arthritis \\
\hline Rac & Ras-related $\mathrm{C} 3$ botulinum toxin substrate \\
\hline RANTES & Regulated on activation normal $\mathrm{T}$ cell expressed and secreted \\
\hline Raptor & Regulatory associated protein of mTOR \\
\hline Rheb & Ras-homolog enriched in brain \\
\hline Rho & Ras homolog gene family \\
\hline Rictor & $\begin{array}{l}\text { Rapamycin-insensitive companion of mammalian target of } \\
\text { rapamycin }\end{array}$ \\
\hline RNA & Ribonucleic acid \\
\hline ROCK & Rho kinase \\
\hline ROS & Reactive oxygen species \\
\hline $\mathrm{rpS6}$ & Ribosomal protein S6 \\
\hline RT-PCR & Reverse transcription-polymerase chain reaction \\
\hline SDF & Stromal derived factor \\
\hline SDS & Sodium dodecyl sulphate \\
\hline SDS-PAGE & Sodium dodecyl sulphate-polyacrylamide gel electrophoresis \\
\hline SH2 & Src-homology 2 \\
\hline SHIP & Src-homology 2 domain-containing inositol phosphatase \\
\hline S6K & S6 kinase \\
\hline Stat & Signal transducer and activator of transcription \\
\hline
\end{tabular}




\begin{tabular}{ll} 
TAM & Tumor associated macrophages \\
TBS & Tris buffered saline \\
TCR & T cell receptor \\
Th & T helper \\
TIL & Tumor infiltrating T lymphocytes \\
TLR & Toll-like receptor \\
TM & Trans-membrane \\
TNF $\alpha$ & Tumor necrosis factor $\alpha$ \\
TNFR & Tumor necrosis factor receptor $\alpha$ \\
TOP & Tract of oligopyrimidines \\
TRAIL & TNF-related apoptosis-inducing ligand \\
TSC & Tuberous sclerosis complex \\
TXP & Threonine-X-Proline \\
UTR & Untranslated region \\
VACV & Vaccinia virus \\
VARV & Variola virus \\
VCAM & Vascular cell adhesion molecule \\
VCP & Vaccinia virus complement control protein \\
VEGF & Vascular endothelial growth factor \\
VLA & Very late antigen \\
WASp & Wiskott-Aldrich syndrome protein \\
WAVE/Scar & World health organization \\
WCR & Vaccinia virus strain Western Reserve \\
XCL & Xine receptor \\
XCR & Xetigand \\
\hline
\end{tabular}




\section{Chapter 1}

\section{Introduction}

\section{A portion of this chapter was published as:}

Galligan C.L., Murooka, T.T., Rahbar, R., Baig, E., Majchrzak-Kita, B., and Fish, E.N. (2006). Interferons and viruses: signalling for supremacy. Immunol Res 35, 27-40. 


\subsection{Chemokine Superfamily}

\subsubsection{Chemokines classification}

Chemokines, or chemotactic cytokines, represent a family of over 40 small proteins (70-120 amino acid residues, 8-17 kd molecular mass range) that, for the most part, are secreted into the environment and function by binding to $G$ protein-coupled receptors (GPCRs)(494). When initially identified close to 30 years ago, these molecules were associated with various human inflammatory diseases and it was thought that their expression might be integral to leukocyte recruitment to inflamed tissue. Within a relatively short period of time, early participants within the field determined that these proteins exhibit distinct and conserved structural features and exert potent chemotactic effects on defined lymphocyte subsets. X-ray crystallography and NMR spectroscopy revealed that these molecules share a tertiary structures framework, organized around a core domain with three antiparallel $\beta$-strands $\left(\beta_{(1)}, \beta_{(2)}\right.$ and $\left.\beta_{(3)}\right)$ overlaid by a C-terminal $\alpha$-helix $\left(\alpha_{(\mathrm{C})}\right)$. Upstream of the $\beta$-sheet is the $\mathrm{N}$-terminal region, which consists of a more or less disordered and extended region of 6-10 amino acid residues, followed by a long loop (N-loop), and a short 3.10 helix. Disulfide bonds involving in N-terminal and core domain cysteines (Cys) stabilize the overall structure $(21,130,144)$.

According to the relative position of Cys residues, chemokines have been classified in four families: CXC- $(\alpha$-chemokines), $\mathrm{CC}$ - ( $\beta$-chemokines, $\mathrm{C}$ - $(\gamma$-chemokine $)$ and CX3C- ( $\sigma$-chemokine)(467) (Table 1). The CC and CXC (X indicates any amino acid) are the 2 largest of these groups. The CXC family members have an intervening amino acid between the first two Cys and are all located on chromosome $4(393,460)$. They primarily stimulate neutrophils, although they are capable of stimulating other leukocyte 
subsets. The receptor-binding site of the CXC chemokines is located in the $\mathrm{N}$-terminal domain. These chemokines may be further classified as Glu-Leu-Arg ("ELR") and "nonELR", based on the presence or absence of this motif before the first Cys $(128,261)$. CC chemokines are all located on chromosome 17 and the first two cysteines are adjacent. CC chemokines stimulate monocytes, basophils, eosinophils, T-lymphocytes, and NK cells. Two highly related chemokines have been identified for the $\mathrm{C}$, and one (Fractalkine) for the CX3C groups. Fractalkine has three residues between the first two cysteins and the chemokines that lack the first conserved cysteine residue are referred to as either C-chemokines or XC-chemokines $(257,507,556)$.

Chemokines are important effectors in numerous biological processes ranging from maintaining the organizational integrity of secondary lymphoid tissue to participating in various aspects of both innate and adaptive immune responses following microbial infection. Accordingly, chemokines have been classified into inflammatory or homeostatic molecules based on their patterns of expression and associated functions $(405,445,446)$. Inflammatory chemokines are produced by activated cells and recruit leukocytes to inflamed tissues, whereas homeostatic chemokines are constitutively produced and involved in maintaining homeostatic leukocyte trafficking as well as the architecture of secondary lymphoid organs. Inflammatory mediators such as tumor necrosis factor (TNF), interferon- $\gamma($ IFN- $\gamma)$, microbial products, or trauma may trigger expression of inducible chemokines. These chemokines have roles in both innate and adaptive immunity in response to infection, tissue damage, and other physiological abnormalities. Their expression is transient, until resolution of the insult. Homeostatic chemokines coordinate the continuous basal level of cell migration needed for the proper 
function of the immune system $(99,446,560)$. They are expressed in bone marrow and lymphoid tissues and are involved in hematopoiesis, immune surveillance, adaptive immune responses and homing to particular anatomic compartments (446). However, this distinction should not be regarded as absolute, but rather as a rule with some exceptions, as some chemokines fall into both categories depending on the biological context. A precise regulation of leukocyte response to the inducible and constitutively expressed chemokine must be critical for maintaining homeostasis of the immune system. It is possible that factors which modify or interfere with leukocyte response to chemokines "confuse" leukocytes and affect immune responses, including inflammation, autoimmune diseases, and organ-transplant rejection.

To deal with the proliferation of synonyms in chemokines, A. Zlotnik and O. Yoshie developed a systematic chemokine nomenclature, based on protein structure and a previous terminology for chemokine gene loci, and proposed it at the Keystone Symposium on Chemokines and Chemokine Receptors, Keystone, CO, 1999. For example, CCL2 refers to a chemokine ligand of the $\underline{\mathrm{CC}}$ subfamily, number $\underline{2}$. This particular chemokine was previously called MCP-1, or monocyte chemoattractant protein 1. At present, the systematic names refer to human chemokines, in part because of uncertainties regarding the identity of mouse orthologs. Table 1-1 includes accession numbers and common names for putative mouse orthologs. In cases such as CCL6, CCL9, and CCL12, where a mouse chemokine lacks a known human ortholog, the standard name is reserved for the potential human counterpart, although it may not exist due to lineage-specific gene duplication. In many cases, the same common name applies to human and mouse counterparts(786). 
Table 1-1

The chemokine superfamily, nomenclature and common names for putative mouse orthologs 


\begin{tabular}{|c|c|c|c|c|}
\hline \multicolumn{5}{|c|}{ Official Name } \\
\hline & & & Common Synonyms & Other Names \\
\hline \multicolumn{5}{|l|}{$\begin{array}{l}\text { Protein } \\
\text { CXC }(\alpha)\end{array}$} \\
\hline \multicolumn{5}{|l|}{ Chemokines } \\
\hline CXCL1 & GRO1 & $4 \mathrm{q} 21$ & GRO; MGSA; (mouse) N51/KC; (mouse) MIP-2 & SCYB1; NAP-3; GRO1 oncogene \\
\hline CXCL2 & $G R O 2$ & $4 q 21$ & Gro; MIP-2 & SCYB2; GRO2 oncogene \\
\hline CXCL3 & GRO3 & $4 \mathrm{q} 21$ & Gro; MIP-2 & SCYB3; GRO3 oncogene \\
\hline CXCL4 & PF4 & $4 q 12-q 13$ & Platelet factor-4 & SCYB4 \\
\hline CXCL5 & SCYB5 & $4 q 13-q 21$ & ENA-78 & SCYB5 \\
\hline CXCL6 & SCYB6 & $4 q 21$ & GCP-2 & SCYB6 \\
\hline CXCL 7 & $P P B P$ & $4 q 12-q 13$ & PBPCTAP-III-TGNAP-2 & SCYB7; low-affinity platelet factor- 4 \\
\hline CXCL8 & $I L 8$ & $4 q 12-q 13$ & IL-8 & $\begin{array}{l}\text { SCYB8; MDNCF; NAP-1; LYNAP; NAF; } \\
\text { GCP-1 }\end{array}$ \\
\hline CXCL9 & MIG & $4 q 21$ & Mig & SCYB9; (mouse) CRG-10 \\
\hline CXCL10 & INP 10 & $4 q 21$ & IP-10; (mouse) CRG-2 & SCYB10 \\
\hline CXCL11 & N.A. & $4 \mathrm{q} 21.2$ & I-TAC; -R1, IP9, H174 & SCYB11 \\
\hline CXCL12 & SDF1 & $10 \mathrm{q} 11.2$ & SDF-1; SDF-1; PBSF & SCYB12; TPAR 1 ; TLSF \\
\hline CXCL13 & N.A. & $4 \mathrm{q} 21$ & BCA-1; BLC & SCYB13 \\
\hline CXCL14 & N.A. & $5 q 31$ & BRAK; bolekine & SCYB14 \\
\hline \multicolumn{5}{|l|}{$\operatorname{CC}(\beta)$} \\
\hline \multicolumn{5}{|l|}{ Chemokines } \\
\hline CCL1 & SCYA1 & 17 & I-309; (mouse) TCA-3 & (Mouse) SIS-f \\
\hline CCL2 & SCYA2 & $17 \mathrm{q} 11.2-\mathrm{q} 12$ & MCP-1; MCAF; (mouse) JE & $\mathrm{HC} 11$ \\
\hline CCL3 & $S C Y A 3$ & $17 q 11-q 21$ & MIP-1; MIP-1S; LD78 & GOS19-1; PAT 464.1; TY-5; SIS \\
\hline NA & SCYA3L1 & $17 \mathrm{q} 11-\mathrm{q} 21$ & LD78, MIP-1P & GOS19-2; PAT 464.2 \\
\hline CCL4 & SCYA4 & $17 \mathrm{q} 11-\mathrm{q} 21$ & MIP-1 & $\begin{array}{l}\text { ACT-2; PAT 744; H400; SIS-; LAG-1; } \\
\text { HC21; G-26; MAD-5 }\end{array}$ \\
\hline CCL5 & SCYA5 & $17 \mathrm{q} 11.2-\mathrm{q} 12$ & RANTES & SIS- \\
\hline CCL6 (reserved) & SCYA6 & & (Mouse) $\mathrm{C} 10$; (mouse) MRP-1 & \\
\hline CCL7 & SCYA7 & $17 \mathrm{q} 11.2-\mathrm{q} 12$ & MCP-3 & NC28; FIC; (mouse) MARC \\
\hline CCL8 & SCYA & $17 q 11.2$ & MCP-2 & $\mathrm{HC} 14$ \\
\hline CCL9 (reserved) & SCYA9 & & (Mouse) MRP-2; (mouse) MIP-1 & (Mouse) CCF18 \\
\hline CCL10 (reserved) & SCYA10 & & & \\
\hline CCL11 & SCYA11 & $17 \mathrm{q} 21.1-\mathrm{q} 21.2$ & Eotaxin & \\
\hline CCL12 (reserved) & SCYA12 & & (Mouse) MCP-5 & \\
\hline CCL13 & SCYA13 & $17 \mathrm{q} 11.2$ & MCP-4 & Ck10; NCC-1 \\
\hline CCL14 & $S \subset Y A 14$ & $17 \mathrm{q} 11.2$ & $\begin{array}{l}\text { CC-1; HCC-1; NCC-2; CCCK-1/CCCK-3; Ck1; } \\
\text { MCIF }\end{array}$ & \\
\hline CCL15 & $S \subset Y A 15$ & $17 \mathrm{q} 11.2$ & $\begin{array}{l}\text { HCC-2; leukotactin-1 (Lkn-1); MIP-5; CC-2; } \\
\text { NCC-3; MIP-1 }\end{array}$ & \\
\hline CCL16 & $S C Y A 16$ & $17 q 11.2$ & $\begin{array}{l}\text { HCC-4; LEC; NCC-4; LMC; monotactin-1 (Mtn- } \\
\text { 1); LCC-1; ILINCK }\end{array}$ & \\
\hline CCL17 & SCYA17 & $16 \mathrm{q} 13$ & TARC & STCP-1 \\
\hline CCL18 & SCYA18 & $17 \mathrm{q} 11.2$ & DC-CK-1; PARC; MIP-4; AMAC-1; ck7 & \\
\hline CCL19 & SCYA19 & $9 \mathrm{p} 13$ & MIP-3; ELC; exodus-3; ck11 & \\
\hline CCL20 & SCYA20 & $2 q 33-q 37$ & MIP-3; LARC; exodus-1; (mouse) ST38 & \\
\hline CCL21 & SCYA21 & $9 \mathrm{p} 13$ & 6Ckine; SLC; exodus-2; TCA4; ck9 & \\
\hline CCL22 & SCYA22 & $16 \mathrm{q} 13$ & MDC; (mouse) dc/-ck; (mouse) abcd-1 & STCP-1 \\
\hline CCL23 & SCYA23 & $17 \mathrm{q} 11.2$ & MPIF-1; MIP-3; ck8-1 & \\
\hline CCL24 & SCYA24 & $7 \mathrm{q} 11.23$ & MPIF-2; eotaxin-2; ck6 & \\
\hline CCL25 & SCYA25 & & TECK, ck15 & \\
\hline CCL26 & SCYA26 & $7 \mathrm{q} 11.23$ & Eotaxin-3; MIP-4 & \\
\hline CCL27 & SCYA27 & $9 \mathrm{p} 13$ & ESkine; CTACK; ILC (mouse) ALP; skinkine & \\
\hline \multicolumn{5}{|l|}{$C(\gamma)$ Chemokines } \\
\hline XCL1 & $S C Y C I$ & $1 \mathrm{q} 23$ & Lymphotactin ; SCM-1; ATAC & \\
\hline XCL2 & $S C Y C 2$ & $1 \mathrm{q} 23-\mathrm{q} 25$ & Lymphotactin; SCM-1; ATAC & \\
\hline \multirow{2}{*}{\multicolumn{5}{|c|}{$\begin{array}{l}\mathrm{CX}_{3} \mathrm{C}(\delta) \\
\text { Chemokine }\end{array}$}} \\
\hline & & & & \\
\hline $\mathrm{CX}_{3} \mathrm{CL} 1$ & SCYD 1 & $16 \mathrm{q} 13$ & Fractalkine; (mouse) neurotactin & $\mathrm{CX}_{3} \mathrm{C}$ ligand \\
\hline
\end{tabular}




\subsection{Chemokine receptors}

Members of a family of 7-transmembrane-spanning, G-protein-coupled receptors mediate the specific effects of chemokines in their target cells $(405,564)$. Chemokine receptors belong to the superfamily of G-protein-coupled receptors that include receptors for hormones, neurotransmitters, paracrine substances, inflammatory mediators, certain proteinases, taste and odorant molecules, photons and calcium ions (735). To date 20 human chemokine receptors (plus three atypical chemokine receptors) have been identified (Table 1-2), which share many common structural features. They are $\sim 40 \mathrm{kd}$ proteins and are comprised of $\sim 350$ (339 to 373) amino acids. The short, disordered and acidic N-terminus followed by three extracellular hydrophilic loops (ECLs) is exposed outside the cell, whereas the C-terminus and three intracellular loops (ICLs) face the cytoplasm. The intracellular C-terminus containing serine and threonine residues are important for receptor desensitization and have phosphorylation sites that are important for receptor regulation. The first two extracellular loops of chemokine receptors are linked together by disulfide bonds between two conserved Cys residues. In addition, the presence of a disulfide bond between the second and third ECL is a unique characteristic of the GPCR superfamily (495). The N-terminus end of the chemokine receptor binds to chemokine(s) and is important for ligand specificity. All chemokine receptors contain a conserved DRYLA(I)A motif in their second ICL. The ability of chemokine receptors to signal upon ligand binding is due, at least in part, to the presence of DRY motif $(49,129$, 197). Subsequent to their activation, chemokine receptors promotes an array of cellular events associated with cell/leukocyte movement, including integrin activation, cytoskeletal reorganization, degranulation, and the generation of oxygen radicals and 
bioactive lipids $(42,44)$.

Chemokine receptors are classified following the chemokine convention as CCR, CXCR, XCR (CR), and $\mathrm{CX}_{3} \mathrm{CR}$ based on the chemokine(s) they bind (281) (Table 1.2). In the new system of nomenclature of chemokine receptors, subfamily $\mathrm{R}$ and an identifying number refer to the receptors; thus, one of the receptors of CCLL2 is CCR22. Although there are over 40 chemokines, there are considerably fewer receptors. Among the 7 receptors that selectively bind to certain CXC chemokines, are chemokine receptors CXCR1 to CXCR7, whereas the CC receptor family consists of 10 receptors, CCR1 to CCR10 $(279,576)$. For fractalkine, $\mathrm{CX}_{3} \mathrm{CR} 1$ is the cognate receptor, and for lymphotactin, the receptor is XCR1. An alternative high-affinity receptor exists for CCL19, CCL21 and CCL25, called CCR11, CCX-CKR or CCRL1 $(235,327,607,608$, 683). CCX-CKR cannot couple to typical chemokine receptor signaling pathways or mediate chemotaxis, and its function remains unclear (683). These properties place CCXCKR in a subfamily of "atypical chemokine receptors" with D6 and Duffy antigen receptor for chemokines (DARC): promiscuous pro-inflammatory chemokine receptors that also appear to be "silent". These receptors bind to chemokines and remove them from the inflammatory milieu $(134,406)$. DARC has been shown to bind to the members of both CC and CXC chemokine subclasses (668). Interestingly DARC binds to angiogenic $\left(\mathrm{ELR}^{+}\right) \mathrm{CXC}$ chemokines but not to angiostatic (ELR') CXC chemokines. Other than DARC, however, ELR ${ }^{+}$CXC chemokines bind only to CXC chemokine receptor 1 (CXCR1) or CXCR2, both of which are expressed on vascular endothelial cells (463, 586). D6 chemokine receptor binds to at least 13 chemokines (CCL2, CCL3, CCL3L1, CCL4, CCL4L1, CCL5, CCL7, CCL8, CCL11, CCL13, CCL14, CCL17 and CCL22) 
with high affinity (Table 1-2) $(477,478)$ and might dampen inflammation in vivo $(239)$. 
Table 1-2

Chemokine receptors and their ligands 


\section{Systematic name}

CXC chemokines

CXCL1

$\mathrm{CXCL2}$

CXCL3

CXCL4

CXCL5

CXCL6

CXCL7

CXCL8

CXCL9

CXCL10

CXCL11

CXCL12

CXCL13

CXCL14

CXCL15

CXCL16

C chemokines

XCL1

XCL2

$\mathrm{CX}_{3} \mathrm{C}$ chemokines $\mathrm{CX}_{3} \mathrm{CL} 1$

CC chemokines

CCL1

CCL2

CCL3

CCL3L1

CCL4

CCL5

CCL6

CCL7

CCL8

CCL9/CCL10

CCL11

CCL12

$\mathrm{CCL} 13$

CCL14

CCL15

CCL16

CCL17

CCL18

CCL19

CCL20

CCL21

CCL22

CCL23

CCL24

CCL25

CCL26

CCL27

CCL28
Original ligand name

GROa

GRO $\beta$

GRO $\gamma$

PF4

ENA-78

GCP-2

NAP-2

IL-8

Mig

IP-10

I-TAC

SDF- $1 \alpha / \beta$

BCA-1

BRAK

Unknown

-

Lymphotactin/SCM-1 $\alpha$ SCM-1 $1 \beta$

Fractalkine

I-309

MCP-1

MIP-1 $\alpha$

LD78 $\beta$

MIP- $1 \beta$

RANTES

Unknown

MCP3

MCP-2

Unknown

Eotaxin

Unknown

MCP-4

HCC-1

HCC-2/Lkn-1/MIP-18

HCC-4/LEC/LCC-1

TARC

DC-CK1

MIP-3 $\beta / E L C$

MIP-3 $\alpha /$ LARC

6Ckine/SLC

MDC

MPIF-1/CKb8

Eotaxin-2

TECK

Eotaxin-3

CTACK

MEC
Receptors

CXCR2, CXCR
CXCR2
CXCR2
Unknown
CXCR2
CXCR1, CXCR2
CXCR2
CXCR1, CXCR2
CXCR3
CXCR3
CXCR3
CXCR4
CXCR5
Unknown
Unknown
CXCR6

XCR1

XCR1

$\mathrm{CX}_{3} \mathrm{CR} 1$

CCR8

CCR2

CCR1, CCR5

CCR1, CCR5

CCR5

CCR1, CCR3, CCR5

Unknown

CCR1, CCR2, CCR3

CCR3, CCR5

CCR1

CCR3

CCR2

CCR2, CCR3

CCR1, CCR5

CCR1, CCR

CCR1, CCR2

CCR4

Unknown

CCR7

CCR6

CCR7

CCR4

CCR1

CCR3

CCR9

CCR3

CCR10

CCR3/CCR10 
In contrast to the cytokine receptor system, different chemokines are capable of binding to a single receptor (e.g. CXCL9, CXCL10, and CXCL11 bind to CXCR3) and a single chemokine may be to multiple receptors within, and sometimes outside, the respective subfamily that it specifically binds (e.g. CCL5 may bind to CCR1, CCR3, and CCR5)(383, 386). Notably, many cell types express multiple chemokine receptors and produce different chemokines, so that if one ligand or receptor is defective, an alternate set of chemokines and their receptors accomplishes the biological function. For example, eight different CXC chemokines can bind and activate CXCR2. However, if CXCR2 is defective or absent, neutrophils can still be recruited through CXCL8 (IL-8) or GCP-2 activation of CXCR1. Evidence suggests that in some cases ligand redundancy does not mean duplicity of functions and different chemokines interacting with the same receptor may activate different sets of signaling pathways, leading to distinct cellular responses (163). In vitro, studies comparing the activities of the two CCR7 ligands, CCL19 and CCL21, revealed differences in their affinity, their ability to desensitize the receptor (56), and the signaling pathways they induce (334). These results indicate that the roles of the two ligands are not merely redundant. Similar findings have recently been reported for the two ligands of human CCR4 and those of mouse CXCR2 (674). The results suggest that different ligands for the same receptor act in different compartments; one ligand may act at the endothelial surface to promote vascular recognition, and the other chemokine would engage the receptor within the tissue microenvironment to guide cellular localization. As expected, the ligand acting in the vascular compartment always exhibits a low capacity to desensitize the receptor, to allow cells to respond to the second stimulus $(56,408,674)$. Moreover, some chemokines act as agonists for some receptors and as 
antagonists for others $(149,487,488,573)$.

On the basis on their function, chemokine receptors can be classified in an "inflammatory or inducible" group, which is activated under pathological conditions, and a "constitutive or housekeeping" group, taking care of re-circulation and maturation of lymphocytes in the unchallenged host, yet also capable of another function (Figure 11)(42). For example, CCR6 is constitutively expressed in immature dendritic cells (DCs) and in T cells, but it is downregulated as DCs mature. However, it is upregulated in skin lesions. CCR8 is constitutively expressed in the thymus but is upregulated during T cell activation and is a marker of Th2 responsive cells. In addition, CCR7 is upregulated during the antigen recognition process, a requisite for immune surveillance, because it is upregulated as DCs mature. Accordingly, chemokine receptor classification into 'specific' or 'shared' is defined by whether they bind a single or many ligands, respectively.

But despite their crucial roles in guiding the recruitment and positioning of leukocytes in the immune system tissues, chemokines and their receptors are associated with numbers of pathologies, which is a hallmark of many autoimmune (e.g., psoriasis, rheumatoid arthritis, and multiple sclerosis), chronic inflammatory (e.g. asthma and chronic obstructive pulmonary disease), and vascular diseases, transplant rejection and cancer (Figure 1-1)(43, 50-52, 120, 216, 228, 236, 346, 348, 526, 712).

In addition, several chemokine receptors can function as viral receptors and coreceptors during the course of infection. CCR5 is likely the most physiologically important co-receptor, having a major role in viral transmission and pathogenesis (276, $390,654,778$ ). Human immunodeficiency virus (HIV) uses CCR5 or CXCR4, as coreceptors to enter target cells. 


\subsection{Chemokine-chemokine receptor binding and signal transduction}

Mutational, structural and functional studies of CCR5 have revealed that the Nterminus residues located before the first Cys and the N-loop residues between the second Cys and the 3.10 helix are involved in chemokine binding. The generation of functional receptor chimeras have allowed for the identification of motifs determining ligandbinding selectivity in chemokine receptors. Specifically, the extracellular regions of chemokine receptors are the major determinants of ligand binding and that the Nterminus dictates this selectivity (389). Furthermore, La Rosa et al showed that the exchange of the extracellular N-terminus domains between CXCR1 and CXCR2 switched the ligand binding selectivity (362). Ligand binding and receptor activation rely on a two-step mechanism, by which affinity and binding specificity are determined by residues in the $\mathrm{N}$-loop region, which bind to the receptor $\mathrm{N}$-terminus, while receptor activation and signaling are triggered by the chemokine $\mathrm{N}$-terminus which interacts with the extracellular loops 2 or 3 of the receptor (525). 
Figure 1-1

Chemokine receptors and their role in diseases 
Figure 1.1

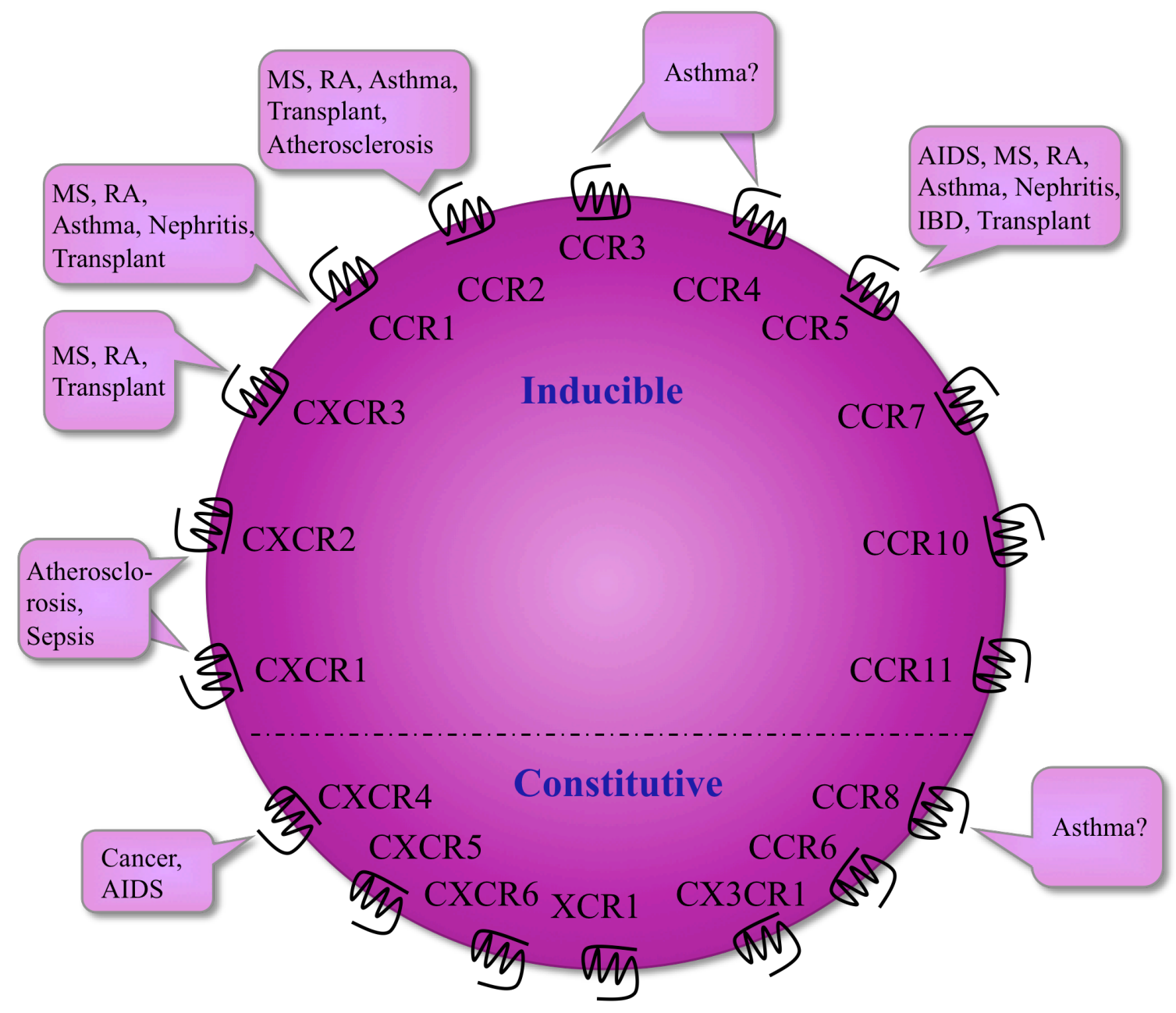




\subsubsection{Chemokine-glycosaminoglycan (GAG) binding}

In addition to chemokine-chemokine receptor interactions, chemokines bind via electrostatic interactions with negatively charged soluble glycosaminoglycans (GAGs) (721), as well as GAGs immobilized on cell surfaces and the extracellular matrix (578). GAGs are linear heterogeneous sulfated polysaccharide chains that are covalently attached to core proteins, forming the proteoglycan family, and are ubiquitously present on all animal cell surfaces and in the extracellular matrix (68). GAGs can be classified into four major families: the hyaluronic acid, the chondroitin/dermatan sulfate, the heparan sulfate/heparin, and the keratan family. Subtle variations in stereochemistry, length, and patterns of sulfation differ between and within GAG families (332).

The heparin-binding properties of many chemokines have been demonstrated; this includes CXCL8 (IL-8) (350, 537, 648, 738), CXCL4 (platelet factor-4) (419), CXCL10 (interferon- $\gamma$ inducible protein-10) (392), CCL4 (MIP-1 $\beta)(221,672)$, CCL3 (MIP-1 $\alpha$ ) (338), and CCL5 (RANTES) (221). The binding sites of chemokines for GAGs and specific chemokine receptors are distinct, such that specificity in these interactions could influence the apparent redundancy (255). The $X \mathrm{BB} X \mathrm{~B} X$ and $X \mathrm{BBB} X X \mathrm{~B} X$ motifs, where B is a basic amino acid and $X$ could be any amino acid, are common GAG-binding motifs for several chemokines (527). Although chemokine-GAG interactions do not seem to activate cell signaling, they appear to be critical for the presentation of chemokines to their cognate receptors (19). This interaction occurs both in vitro and in vivo, and is required for chemokine function in vivo $(255,313,529)$. Chemokines exhibit moderate to high affinity for GAGs, mediated by GAG sulphated, anionic sequences and chemokine basic amino acid domain interactions $(18,113)$. The affinity for different GAG families 
as well as their tendency to aggregate will increase the local concentration of a specific chemokine, thereby inhibiting the diffusion of chemokines away from their sites of production, a characteristic that is necessary for the chemotactic migration of cells towards chemokines $(437,577,750)$. GAG expression varies for different cell types and is also modified during inflammation. These GAG fluctuations will influence the local concentration of a particular chemokine and might control the migration of specific populations of cells and thereby determine which leukocyte subsets enter which tissues $(349,504)$. Moreover, for some chemokines, e.g. CCL5, oligomerization seems to be required for proper activation and function, and GAGs expressed on the endothelial cell surface are thought to contribute $(529,776)$. There is evidence that soluble heparin reduces inflammation by inhibiting the interaction between chemokines and GAGs and subsequently disrupting chemokine aggregation (507). Therefore, determining the GAGbinding profiles of chemokines would be a critical step in developmening novel GAG mimetics with anti-inflammatory activity. These molecules would act by blocking the formation of chemokine gradients on cell surfaces.

CCL5 is a chemoattractant of monocytes, eosinophils, and activated CD4 T cells (786), and binds to CCR1, CCR3, and CCR5. CCL5, like other chemokines, also binds to GAGs at its major GAG binding site, the $\mathrm{BB} X \mathrm{~B}$ motif, where $\mathrm{B}$ represents either of the basic amino acids arginine or lysine, in the $40 \mathrm{~S}$ loop $(410,465,528)$. Although CCL5 exhibits selectivity in GAG binding, with the highest affinity (nanomolar range) for heparin $(410,528)$, the affinity interaction of CCL5 with GAGs is diminished by the addition of chondroitin sulfate, and dermatan sulfate as well $(349,465)$. 


\subsubsection{Chemokine mediated signal transduction}

As mentioned, chemokine receptors are part of a superfamily of G-proteincoupled receptors that include receptors for hormones, neurotransmitters, paracrine factors, inflammatory mediators, certain proteinases, taste and odorant molecules, and even photons and calcium ions. Ultimately, the G-protein-coupling specificity of each receptor determines the nature of its downstream signaling targets (474). G-proteins exist as a heterotrimer and are composed of three distinct subunits: $\mathrm{G} \alpha$, which carries the binding site for the nucleotide, G $\beta$ and G $\gamma$. In the inactive state, G $\alpha$ has GDP in its binding site. Upon activation, a tertiary structure change takes place in the receptor and this triggers an allosteric change in the G $\alpha$ subunit causing GDP to leave and be replaced by GTP. GTP activates G $\alpha$ causing it to dissociate from $\mathrm{G} \beta \mathrm{G} \gamma(252)$, or rearrangement of the heterotrimers (96). Activated $\mathrm{G} \alpha$ in turn activates other effector molecules, including adenylyl cyclase, which is involved in signal transduction $(101,576)$. Furthermore, this activated G $\alpha$ converts its GTP to GDP due to its GTPase activity, which in turn is coupled with the return of the $G \beta$ and $G \gamma$ subunits, restoring the $G$ protein to its inactive state. Different subtypes of $\mathrm{G} \alpha$ include: $\mathrm{G} \alpha_{\mathbf{s}}$ (s for stimulator) which stimulates adenylyl cyclase, $\mathrm{G} \alpha_{\mathrm{q}}$, activating phospholipase $\mathrm{C}$ (PLC), which in turn generates the second messengers inositol-1,4,5-trisphosphate (IP3) and diacylglycerol (DAG), Ga $\alpha_{\mathrm{i}}$ (i for inhibitory) inhibiting adenylyl cyclase, lowering the level of cAMP in the cell and Ga $\alpha_{\mathbf{t}}(\mathbf{t}$ for transducin), the molecule responsible for generating a signal in the rods of the retina in response to light. G $\alpha_{t}$ also triggers the breakdown of cyclic GMP (cGMP) $(101,252$, 474).

Chemokine binding to the G-protein coupled receptors (GPCR), such as CCR2 
and CCR5, also promotes the engagement of G-protein- independent, tyrosine phosphorylation signaling effectors $(70,343,560,758)$. This may result in the association and activation of the Janus tyrosine kinases (JAKs) $(429,756)$ and subsequent recruitment of the JAK-STAT (Signal Transducers and Activators of Transcription) signaling pathway $(756,757)$. Latent monomeric STATs are resident transcription factors in the cytoplasm pre-activation. The seven mammalian STATs contain a conserved tyrosine residue near the C-terminus that is phosphorylated by JAKs (757). In mammals, the JAK family includes four members: JAK1, JAK2, JAK 3 and Tyk2. For most cytokine receptors, ligand binding to the receptor triggers the oligomerization of the receptor, allowing the two receptor-associated JAKs to come into close proximity, which is necessary for their trans-phosphorylation. Once the JAKS are activated kinases, they in turn phosphorylate tyrosine residues in the intracellular domains of the receptor subunits, which then serve as recruitment sites for the STATs. STATs contain a phosphotyrosinebinding Src homology 2 (SH2) domain. Once recruited to the phosphorylated tyrosine residues on the receptor, the JAKs phosphorylate the STATs, which will then dissociate from the receptor, form dimers and translocate to the nucleus via the nucleoprotein interactor 1 (NPI-1) and Ran nuclear import pathway, and then bind to specific regulatory sequences to activate or repress transcription of target genes $(2,262,330$, 486). Phosphorylation-activation of some STAT family members has been reported in T cells post CCL5/ CCL3 stimulation (756). In the PM1 human T cell line CCL5 leads to phosphorylation of JAK2 and JAK3 (757). This phosphorylation is pertussis toxin (PTX) insensitive, indicating that CCL5-CCR5 mediated tyrosine phosphorylation events are not G proteins-dependant (757). 


\subsection{Receptor dimerization}

GPCRs were initially considered to be functionally active as monomers (218). Although the biological significance of GPCR oligomerization is poorly characterized, a mounting body of biochemical and biophysical evidence using a variety of techniques, including immunoprecipitation and fluorescence or bioluminescence resonance energy transfer (BRET) and Fluorescence Resonance Energy Transfer (FRET), indicates that constitutive or ligand-promoted oligomeric complexes, frequently dimers, are essential for signaling $(26,104,263,321,585,779)$. GPCRs can form both homo- and heterodimers, but whether these dimers are stable complexes once formed in the endoplasmic reticulum $(\mathrm{ER})(199,304,498,675)$ or are the targets of ligand-mediated regulation at the cell surface $(27,141,557,561,762)$, remains enigmatic and in some cases even controversial, which indicates the complexity of GPCR activation and signaling in the cell. The regions of GPCRs thought to be involved in oligomerization interfaces include extracellular domains, the transmembrane helices (TMs) and/or the C-terminal domain $(439,440)$. Some GPCRs, such as the metabotropic glutamate receptor-1 (mGluR 1$)$, have a large extracellular region ( $\sim 600$ amino acids in $\mathrm{mGluR}_{1}$ case) that binds to the ligand and contributes to dimer stabilization and signal transmission (686). For other GPCRs such as opioid receptors (OPRM), ligand-induced signal transduction requires interaction between TMs: TM1 and TM2 helices in homodimerization of OPRM (198). For the $\gamma$ aminobutyric acid $(B)\left(G_{A B A}\right)$ receptor, heterodimerization between $G A B A_{B} R 1$ and $\mathrm{GABA}_{\mathrm{B}} \mathrm{R} 2$ occurs through an interaction between their intracellular C-terminus tails, possibly through a conserved coiled-coil domain (743). Chemokine receptor dimerization and its functional implications have described for four different chemokine receptors: 
CCR2, CCR5, CXCR2, and CXCR4 $(39,67,304,430,651,715)$. They are all capable of forming homo-dimers, but hetero-dimers have also been reported between CCR2 and CCR5 (431) and more recently between CCR2 and CXCR4 (646). CCR5-CCR2b heterodimerization increases the sensitivity and dynamic range of the chemokine response (430) and activates hetero-trimeric G-protein $\mathrm{Gq} / 11$, instead of Gi, which is activated by CCR5 or CCR2 alone (430). Furthermore, observations regarding the binding properties of $\mathrm{CCR} 5 / \mathrm{CCR} 2 \mathrm{~b}$ hetero-dimers suggest that hetero-dimers are able to bind to a single chemokine (180). Comparative and phylogenetic analyses documented evidence for gene conversion between the adjacent CCR 2 and CCR 5 genes in Felidae (the biological family of the cats) and in three independent mammalian species; Primates, Cetartiodactyla, and Rodentia. There are high levels of sequence similarity between the two paralogous genes within each order. Interestingly, the gene conversion was restricted to the TM domains of CCR2 and CCR5 (709). This is intriguing since bioinformatic analysis of the chemokine receptor family predicts that TM1, TM2 and TM4 are the principal domains involved in homo-dimer formation (164). For CCR5, isoleucine 52 (Ile52) in TM1 and valine 150 (Val150) have been reported to be critical residues in the interaction between CCR5 molecules (269). It is not surprising, therefore, that the binding site for TAK-779, a small-molecule inhibitor of CCR5, has been mapped to a cavity between TM1, TM2, TM3 and TM7 (172). CXCR4 (517) or CCR5 (663) can also form hetero-dimers with other members of the GPCR family, including the $\delta$-opioid receptor (DOR). Formation of the CXCR4/DOR or CCR5/DOR heterodimers prevents each of them from signaling (198). 


\subsection{Receptor desensitization and endocytosis}

Agonist activation of a GPCR not only initiates the receptor-mediated signal transduction cascade, but also triggers the activation of mechanisms that lead to receptor desensitization and internalization. The mechanisms underlying GPCR internalization have been characterized largely as a result of extensive studies with the prototypical GPCR, the $\beta_{2}$-adrenoceptor $(108,196,407)$. GPCRs internalize via two major mechanisms post ligand binding: a clathrin-coated pit-dependent mechanism, which involves binding of arrestin to the receptor and subsequent receptor internalization (195, $373,374,780)$, and a clathrin-coated pit-independent route, which involves caveolae $(312,479,480,491,516)$. The clathrin-coated pit-dependent pathway is the bestcharacterized mechanism for receptor entry, degradation and recycling (497). Agonist stimulation of GPCRs mediates the activation and subsequent phosphorylation of intracellular residues of GPCRs by GPCR protein kinases (GRKs). This leads to the binding of $\beta$-arrestin to the phosphorylated receptor, which in turn initiates the internalization process by binding to clathrin heavy chain (495). The interaction of ßarrestin with the phosphorylated GPCR desensitizes agonist-mediated signal transduction by preventing further coupling to its cognate $\mathrm{G}$ protein, therefore terminating further signaling from the receptor via $\mathrm{G}$ proteins $(195,518)$. Furthermore, the receptor- $\beta$ arrestin complex is sequestered in clathrin-coated pits, which then become clathrin-coated vesicles via interaction with dynamin (728). Rab5- and rab7-dependent vesicle fusion processes are involved in trafficking of the vesicles from early to late endosomes to lysosomes $(352,534,713,717)$. Once internalized, receptors accumulate in peri-nuclear recycling endosomes and are recycled back to the cell surface in their dephosphorylated 
form (459). Receptor number on the cell surface is a balance between internalization and replacement -recycling and new synthesis.

\subsection{CCR5}

CCR5 is predominantly expressed on macrophages, activated/memory T lymphocytes, DCs, natural killer cells (NKs) and central nervous system (CNS) (neurons, astrocytes, and microglia cells) cells. It is highly expressed in the spleen and thymus, to a lesser extent in the small intestine, and low-level expression is observed in ovaries and lung $(77,211,260,331,541,554,588)$. CCR5 is a functional receptor for a number of pro-inflammatory CC-chemokines, including CCL3 (MIP-1 $\alpha$ ), CCL4 (MIP-1 $\beta)$, CCL5 (RANTES), CCL-8 (MCP-2), CCL11 (eotaxin), CCL13 (MCP-4) and CCL3L1 (LD78ß) $(72,232,493,582,588)$.

The role for CCR5 as a co-receptor for HIV-1 entry identified this chemokine receptor as a potential therapeutic target for HIV. HIV-1 entry is initiated by the interaction between gp120 (the viral envelope glycoprotein), the host cell factor CD4, and one of the co-receptors (377). CCR5 is perhaps the most extensively studied member of the chemokine receptors because of its role as a co-receptor during HIV-1 infection. Most commonly transmitted strains of HIV are strains that bind to CCR5, so called nonsyncytium-inducing (NSI), commonly referred to as R5 strains $(20,159,171)$. Interestingly, in HIV infected individuals, R5 viruses, also called Macrophage- (M-) tropic, are often the dominant strain isolated during the early asymptomatic stages of viral infection $(137,159,547,565,604,785)$, suggesting that these viruses may have a selective advantage during transmission or the acute phase of disease. Utilizing combining protein structure modeling and molecular dynamics simulations, interactions 
such as hydrogen bonds, salt bridges and Van der Waals contacts between CCR5 and gp120 has been described in complex with CD4. It was proposed that CCR5 interacts with gp120 primarily through its negatively charged N-terminus region and the positively charged bridging sheet region of gp120. Furthermore, interactions between ECL2 of CCR5 and the base of the V3 loop regions of gp120 might lead to conformational changes in the gp120 structure and result in HIV entry into the target cell $(380,391,458$, $471,571,631,769)$. The strains of HIV that utilize CXCR4, the other co-receptor for HIV, for entry, are syncytium inducing (SI) or so called X4 strains (188, 244, 310, 344). In contrast to R5, X4 strains, which are also called T cell- (T-) tropic isolates, are typically viruses that are found late in infection and are associated with rapid $\mathrm{CD}^{+} \mathrm{T}$ cell loss $(139,159,309,310)$. It remains unclear whether the emergence and disease progression of $\mathrm{X} 4$ viruses is a consequence of immunodeficiency. In addition, it has been suggested that utilizing CXCR4 as co-receptor alone is not responsible for disease progression, since almost half of the individuals with AIDS continue to harbor predominantly R5 strains $(137,565,604)$. It is believed that R5 strains are transmitted more frequently compared to X4 strains. For instance, the deletion of a 32-bp gene segment in the CCR5 coding region results in a nonfunctional receptor, thus preventing HIV R5 entry. Individuals homozygous for CCR5 32 exhibit resistance to HIV-1 infection $(138,379,509)$, and in HIV infected individuals that are CCR5 32 the spread of infection is also reduced (161). Notably, individuals with higher levels of circulating CCR5-binding chemokines are reported to have reduced ectopic expression of CCR5, probably because of chemokine binding and possible receptor internalization. These individuals are also less susceptible to HIV-1 infection and seem to have a degree of 
immunity against HIV-1 infection (131). To date, a number of small-molecule CCR5 antagonists such as Maraviroc or anti-CCR5 monoclonal antibodies (mAbs) have been developed that demonstrate potent antiviral effects in cell culture and in clinical trials, $(358,403,428,599,685,704,736,760)$.

The human CCR5 gene has been mapped on chromosome 3p21, in a cluster with several other chemokine receptor genes (CCR1, CCR2, CCR3, CCR4, CCR8, CCR9, XCR1, CX3CR1) (467), and encodes a protein of 352-amino acids (Figure 1-2), $40 \mathrm{kd}$ and with a potential N-linked glycosylation site (379). Similar to other 7TM GPCRs, biochemical analysis reveals seven stretches of hydrophobic amino acids, corresponding to seven membrane-spanning $\alpha$-helices, an extracellular N-terminus, an intracellular Cterminus and three extracellular loops: ECL1, ECL2, and ECL3 (781) for CCR5. Amino acid sequence analysis of CCR5 reveals that it belongs to the class A of GPCRs with high homology to the prototypical member of this family, rhodopsin (493). The presence of several tyrosine residues at positions $3,10,14$, and 15 adjacent to a number of acidic amino acids in the $\mathrm{N}$-terminus of CCR5 provides a site for post-translational modification by sulfation $(95,187,574)$. Sulfation of these tyrosines, particularly at positions 3 and 10 , has been shown to facilitate gp120 binding and HIV entry (187). In addition to tyrosine sulfation, CCR5 undergoes an O-linked glycosylation on serine (Ser-) residue-6 (187). There is another N-glycosylation site on the ECL3 of CCR5, a potential site for posttranslational modification, but no evidence for this has been reported. At its C-terminus CCR5 contains three cys residues, sites for thioesterification by palmitate (palmitoylation). This post-translational modification might lead to structural changes in the cytoplasmic tail of CCR5 and promote the formation of a membrane anchor and a 
fourth cytoplasmic loop that could modulate biological activities such as membrane targeting, signaling, endocytosis, and recycling (441). 
Figure 1.2

Schematic representation of CCR5 structure 
Figure 1.2

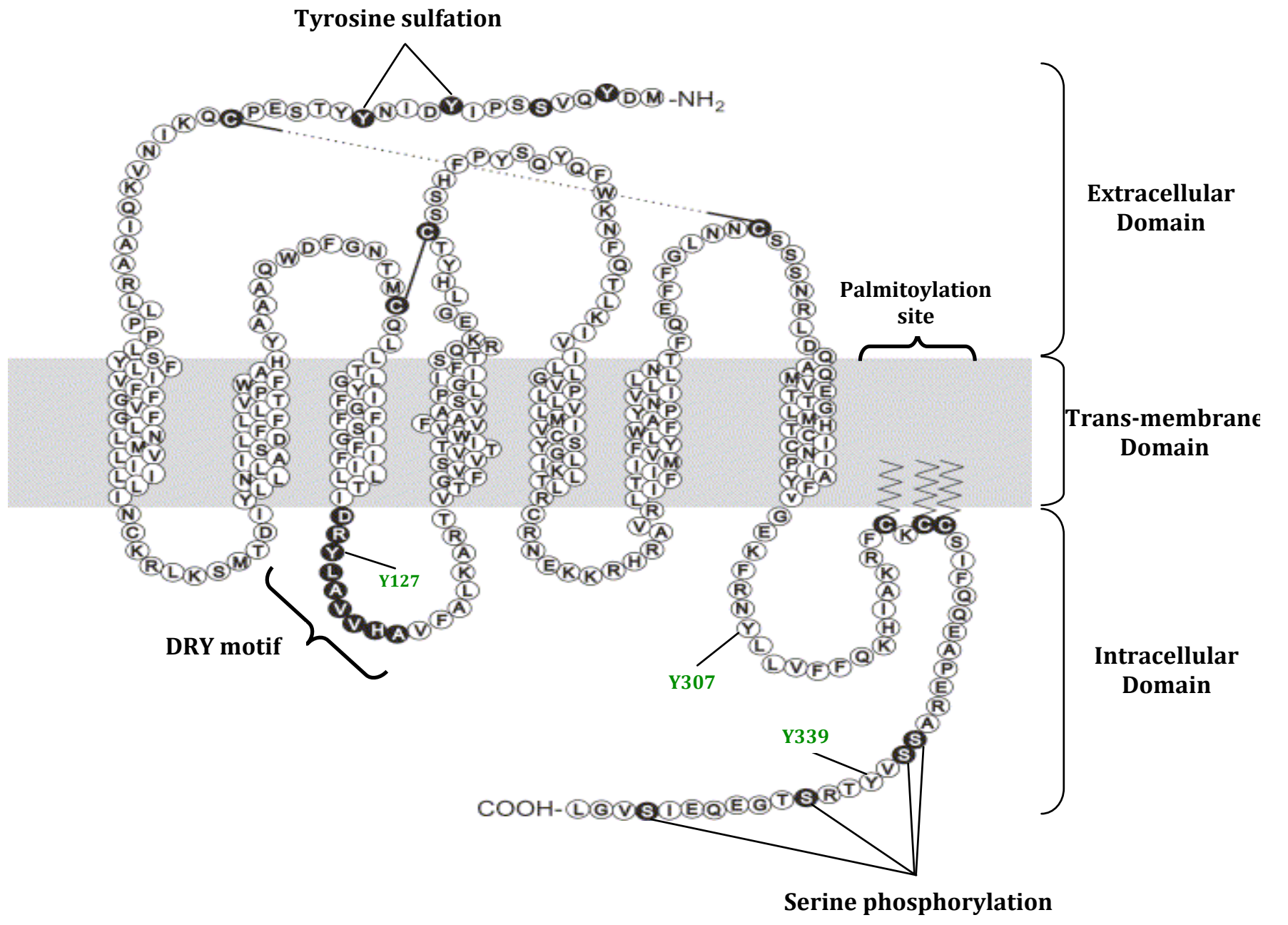

Adapted from M. Oppermann Cellular Signaling 16 (2004) 
Similar to other chemokine receptors, CCR5 possess a unique disulfide bridge between the two conserved cys in its second and third ECLs. Furthermore, two additional cys residues are thought to form another disulfide bond between the N-terminus and ECL3 (Figure 1.2). These disulfide bonds seem to provide the structural integrity necessary for ligand binding and receptor activation, since alanine (ala) or ser substitutions of any of the cys residues resulted in reduced ectopic expression of CCR5 and thereby diminished HIV-1 co-receptor activity and loss of chemokine binding (71, 110). Due to the lack of a crystal structure of CCR5, a precise characterization of the functional domains of CCR5 has been problematic; therefore the development of structure-based CCR5 antagonists for therapeutic clinical indications has been somewhat impeded. However, using three-dimensional (3D) in silico modeling, some insights into the potential interactions between CCR5 and its agonists and antagonists have been obtained. Notably, utilizing 3D quantitative structure-activity relationship (3D-QSAR) methods, Song et al. provided information on molecular features of CCR5-antagonist behavior (647).

Based on amino acid analysis, a two-step mechanism model has been proposed for chemokine binding and activation of CCR5. The primary interaction occurs between the chemokine core and the exposed N-terminal domain of CCR5, then the ligand specific step is the association of the chemokine with region close to the ECL2 of CCR5 (589). Then the free $\mathrm{N}$-terminus domain of the chemokine mediates receptor activation through its interaction with the TM helix bundle $(70,237,495)$. 


\subsubsection{CCR5 mediated signaling}

Receptor dimerization, internalization, recycling, and/or degradation regulate the signaling activity of CCR5 $(431,560)$. Whether CCR5 forms constitutive dimers or the dimerization is a result of agonist modulation, remains to be addressed. Chemokine binding to CCR5 activates both G-protein-dependent signaling as well as PTx-resistant protein tyrosine kinase-dependent pathways $(40,282)$. For CCR5, agonist-inducedinternalization into early endosomes may be mediated via clathrin-dependent endocytosis (399, 628, 629). Using mouse embryonic fibroblasts (MEFs) derived from $\beta$-arrestin knockout mice, evidence was provided that $ß$-arrestin is critical for CCR5 internalization (200), however several publications have suggested that CCR5 may also be internalized through clathrin-independent mechanisms $(457,711)$. Notably, while substitution of ala residues for the three ser residues located at the C-terminus of CCR5 did not affect the binding efficiency of $\beta$-arrestin, CCR5 internalization was impaired and there was a significant deficit in receptor phosphorylation (295). Apparently, caveolae-dependent endocytosis of CCR5 may occur $(457,711)$. There is evidence that palmitoylated Cys residues located at the $\mathrm{C}$-terminus of CCR5 may mediate internalization of the receptor via a clathrin-independent mechanism, into vesicles containing caveolae (711), in contrast to the previous findings that CCR5 palmitoylation is crucial for $\beta$-arrestinmediated receptor internalization (343). During clathrin-dependent internalization, agonist binding mediates clustering of ectopic CCR5 into clathrin-coated regions of the plasma membrane containing the adaptor protein complex 2 (AP2). CCR5 molecules are then internalized through clathrin-coated pits and vesicles into early endosomes together with transferrin, a marker for the clathrin-trigered endocytic pathway. It has been shown 
that induced recruitment of $\beta$-arrestin to the phosphorylated receptor not only desensitizes the receptor from secondary signaling events but also leads to the activation of $\beta$-arrestinmediated signaling such as the mitogen-activated protein kinase (MAPK) cascade (37).

A consequence of CCR5-mediated G protein-coupled signaling, is adenylyl cyclase-PLC $\beta$ activation the subsequent rapid influx of intracellular calcium, and IP3 and DAG formation $(33,783)$. Subsequent activation of PKC induces the rapid phosphorylation of CCR5 on the four C-terminal ser residues by $\mathrm{G}$ protein-coupled receptor kinases (GRK) $(33,343,496,714)$. Experiments with PLC $\beta$-deficient mice revealed that PLC $\beta-2$ and -3 have an important role in CCR5-induced superoxide generation and PKC/MAPK activation (Figure 1.3)(371). In contrast to CCR5-activation of PLC 3 that mediates a rapid calcium influx and activates protein kinase C, CCR5mediated activation of lipid kinase phosphoinositide-3'OH-kinase isoforms, such as phosphatidylinositol-3-kinase (PI3K) $(655,659)$, activates AKT and extracellular signalregulated kinases 1/2 (ERK1/2)(Figure 1.3) (387). PI3K class IB isoform (PI3K $\gamma$ ) and Rho family GTPases have important roles in chemokine-induced leukocytes recruitment. The best-characterized members of the Rho family of GTPases are Rac, Cdc42, and RhoA. Their main role is to couple extracellular signals to actin cytoskeletal rearrangement, in chemokine-mediated cellular events (105). RhoA regulates the formation of focal adhesions and can activate various downstream effectors, including NFK-B, PLC, Rho kinase and MEK (MAP kinase or ERK kinase) kinase, which in turn results in the activation of ERK1/2, p38 MAPK, and c-Jun amino-terminal kinase/stressactivated protein kinase (JNK/SAPK) (210). While PLC $\beta$ deficiency does not affect cell migration, PI3K $\gamma$ deficient mice and those treated with specific PI3K $\gamma$ inhibitors showed 
significantly reduced PI3 production by leukocytes, after stimulation with various chemokines $(274,371)$. Notably, Hirsch et. al. observed a significant reduction in chemokine-mediated neutrophil, monocyte and macrophages recruitment in $\mathrm{PI} 3 \mathrm{~K} \gamma$ deficient mice (274). As mentioned, GPCRs are able to activate kinases independent of G protein activation $(429,431,715)$. Activation of the JAK/STAT pathway has been described in T cells after CCL5 stimulation of CCR5 (756). Notably, in PM1 human T cells CCL5 activation of CCR5 leads to PTx-insensitive phosphorylation of JAK2 and JAK3 (757) and subsequent STAT activation. 
Figure 1.3 Schematic representation of CCL5-CCR5 signaling events 
Figure 1.3

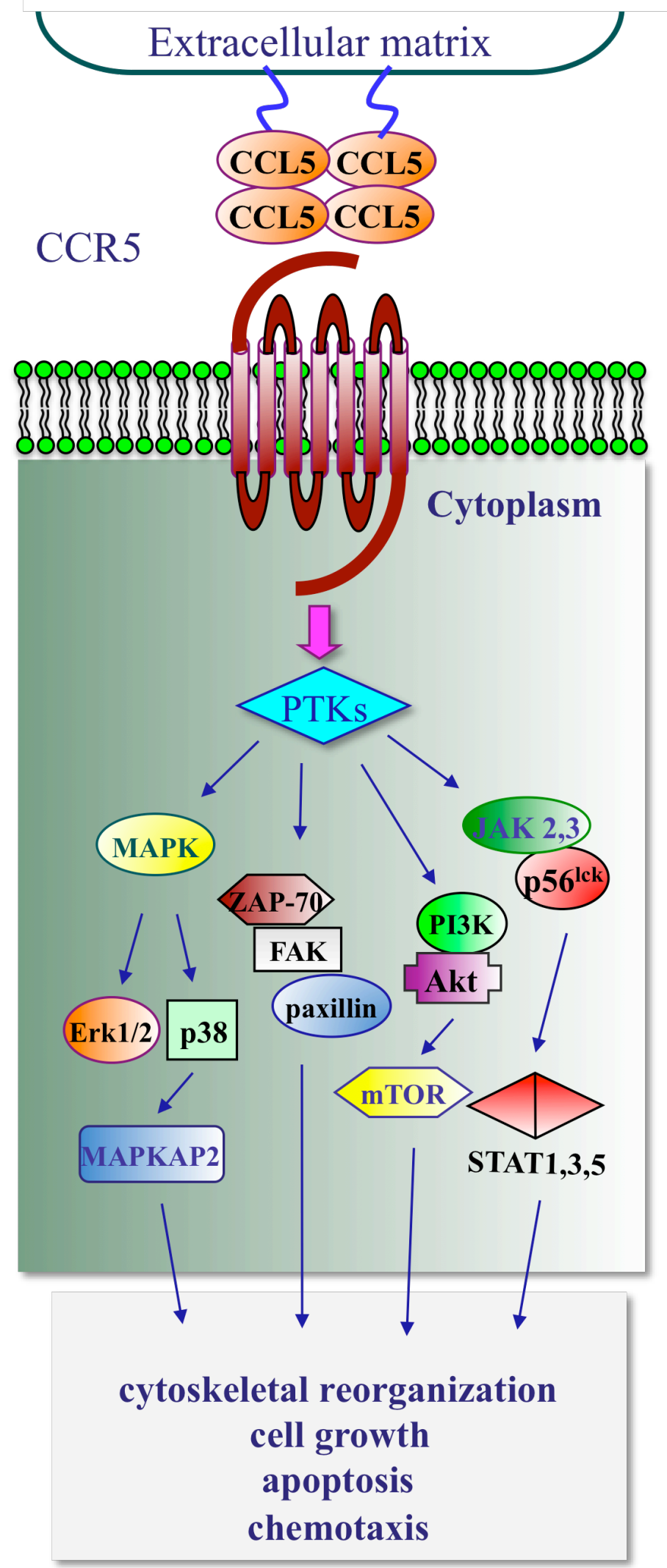




\subsubsection{CCR5: a potential therapeutic target}

Although the major roles for chemokines and their receptors are to orchestrate trafficking and to recruit and activate specific leukocyte subpopulations, the significance of their involvement in the progression and pathophysiology of inflammation in chronic inflammatory disorders, autoimmune diseases and solid organ transplant rejection is becoming increasingly apparent $(47,223-226,292)$. Rheumatoid arthritis (RA) is a chronic inflammatory disease associated with bone and cartilage destruction, that causes progressive loss of mobility (364). In the synovial tissue (ST) of affected rheumatoid joints there are abundant macrophages and T lymphocytes expressing CCR 5 and CXCR3 (333, 470, 716). CCL3, CCL4 and CCL5 are present at high levels in the synovial fluid (SF) of affected RA joints $(508,662,716)$. In vitro experiments have shown that inhibiting CCL3 and CCL5 in RA SF using neutralizing antibodies will inhibit chemotaxis of lymphocytes $(8,333,555,716)$. In vivo experiments using chemokineencoding DNA vaccines enhanced the breakdown of tolerance to CCL5 and CCL3 and was effective in treating ongoing adjuvant arthritis (774). A polyclonal antibody directed against CCL5 improve disease symptoms in a rat adjuvant arthritis model, through a reduction in leukocyte migration into inflamed joints (57). Utilizing TAK-779, Yang et. $a l$. provided evidence for a reduction in both the incidence and the severity of collageninduced arthritis (CIA) in DBA/1 mice, mediated by an inhibition of chemokine regulated leukocyte migration to affected joints (770).

Multiple sclerosis (MS) is a chronic inflammatory disease of the CNS. The pathogenesis of MS is not well understood, but involves aberrant humoral and cellmediated immune responses. A working hypothesis of pathogenesis is that $\mathrm{T}$ cell 
activation occurs in response to macrophage/MHC class II presentation of "myelin mimicking" antigen. Subsequently, activated T cells, which express vascular adhesion molecules, infiltrate into the CNS. There they release pro-inflammatory cytokines, resulting in local CNS microglia functioning as antigen-presenting cells presenting selfmyelin and other myelin-associated proteins to T cells. This results in the further activation of $\mathrm{T}$ cells, promoting an autoimmune inflammatory cascade $(206,761) . \mathrm{T}$ lymphocytes expressing CCR5 and CXCR3 have been reported to be present in active lesions in MS $(47,226)$. Notably, there is evidence that in CCR5 $\Delta 32$ individuals, the progression (prolonged relapse-free intervals, less prominent abnormalities), but not incidence, of MS is reduced (614). In addition, antagonists for CCR1, CCR2, CCR5 and CXCR3 have been considered as therapeutic agents for MS (476). BX-471 (Berlex Biosciences) is one such compound that reversibly binds to CCR1 and competes for CCR1 binding with CCL3, CCL5 and CCL7 (476). UK-427857 (Pfizer) is a small receptor antagonist for CCR5 about to enter clinical trials (669). TAK-779 has also been suggested to have potential beneficial therapeutic effects in MS patients, however poor oral absorption and rapid elimination has limited its usage $(212,461)$.

Increased levels of CCL3, CCL4 and CCL5 have been reported in the inflamed CNS of patients with MS, and in an animal model, experimental autoimmune encephalomyelitis $(\mathrm{EAE})(29,598,693)$. It has been shown that treatment with antiCCL3 antibody not only prevents the development of acute EAE, but also limits the severity of disease (318). Anti- CCL2 antibody administration also reduces the number of relapses in chronic-relapsing EAE $(318,323)$.

Met-RANTES, a CCL5 (RANTES) variant that inhibits CCR5, is effective in 
preventing collagen-induced arthritis in mice, delaying disease onset and ameliorating clinical signs in the DBA/1 mouse model (520). In autoimmune glomerulonephritis, MetRANTES reduced mononuclear cell infiltration(382). Alzheimer's disease (CCR5 has been detected on microglial cells in brain lesions) (46), fungal asthma (CCR5 involved in the development and maintenance of chronic fungal asthma) (603) and acute and chronic rejection in human transplantation (503) are other examples of diseases where inhibition of CCR5 may have therapeutic benefits.

\subsection{Viral subversion of host chemokine/chemokine receptor system}

Although originally considered as pro-inflammatory mediators, it is now widely accepted that chemokines and their receptors are involved in the development of an effective host immune response to viral infection. Not surprisingly, therefore, viruses have evolved to subvert this host-chemokine response. Large DNA viruses such as herpesviruses and poxviruses evade a host immune response through molecular mimicry, encoding of cytokines and their receptors and homologues of chemokines or chemokine receptors $(17,241,636,649)$. Poxviruses are uniquely specialized in subverting the hostchemokine response, encoding a unique set of secreted proteins that bind chemokines with high affinity and block their activity by preventing chemokine activation of cellular receptors (17).

\subsection{Poxviruses}

Poxviruses are large double stranded DNA viruses that are members of the Poxviridae family. 14 poxviruses infect humans. Among them, variola 
(smallpox)(VARV), vaccinia (VACV), cowpox (CPXV), monkeypox (MPXV), buffalopox, cantagalo and aracatuba belong to the Orthopoxvirus genus, orf, paravaccinia, bovine popular stormatitis, deerpox and sealpox belong to the Parapoxvirus genus, molluscum contagiosum belongs to the Molluscipox genus and tanapox to the Yatapoxvirus genus $(127,151,367,368)$. While most human poxvirus infections fail to establish a human chain of transmission, all poxviruses could be transmitted from animals to humans or from humans to animals, with the exception of smallpox and molluscum contagiosum (92-94). Although smallpox, which was eradicated worldwide during the 1970s WHO campaign, is the most virulent human poxvirus pathogen (190), MPXV, orf virus and molluscipox virus are the most common human poxvirus infections. Domestic cats are most frequently responsible for transmission of CPXV to humans, however CPXV may be acquired from cows, sheep or rodents $(605,755)$. Concern regarding the use of VARV as an agent of bioterrorism has led to an increased interest in research studies related to orthopoxviruses, to understand the molecular and cellular bases of pathogenesis for the development of antiviral agents.

\subsection{VARV}

Variola, the causative agent of smallpox, is probably the most well known member of the poxvirus family. Smallpox, Variola or Variola vera, has its roots in the Latin varius, meaning spotted, or varus, meaning pimple. Smallpox was so called to distinguish it from the great pox, syphilis (575). The first evidence of smallpox was found in Egyptian mummies of the $18^{\text {th }}$ and $20^{\text {th }}$ Dynasty (1580-1085 BC). In Asia, historical evidence for a smallpox-like disease dates to 1122 B.C. in China and 1500 B.C. in India 
$(166,186,194)$. In the $4^{\text {th }}$ century A.D. in China, and in the $7^{\text {th }}$ century A.D. in India and the Mediterranean, VARV became endemic and ultimately spread to Europe in the $8^{\text {th }}$ century and to southwestern Asia in the $10^{\text {th }}$ century (194). The use of smallpox as a biological weapon during the French-Indian wars decimated the Native American population significantly in the $15^{\text {th }}$ and $16^{\text {th }}$ centuries. In 1519 , Spanish forces used smallpox against the Aztecs, almost completely annihilating them, causing the population to fall from 25 million in 1519 to three million 50 years later.

Throughout history smallpox outbreaks caused considerable mortality. In 1848, the English historian Thomas Macaulay wrote that the disease "was always present, filling the churchyard with corpses, tormenting with constant fears all whom it had not yet stricken ... making the eyes and cheeks of a betrothed maiden objects of horror to the lover" (396). In the 20th century, smallpox continued to be a major worldwide pathogen, responsible for a half million deaths per year in Europe, making it the most important cause of morbidity and mortality in human history: "the most terrible of the ministers of death" (194). Although declared eradicated in 1980 by the World Health Organization (WHO), the fear of possible outbreaks of wild type/genetically altered smallpox, or the potential for biological weapon still exists. The existence of smallpox stockpiles in the U.S. and Russia, generated for bio-weapons research during the cold war, raises the possibility of the virus falling into the hands of terrorists.

\subsubsection{Smallpox Pathogenesis and Symptoms}

Smallpox is a highly contagious disease and infection always results in symptomatic disease. Inoculation with VARV usually occurs through the oropharyngeal 
or respiratory mucosa, however in some cases virus has been shown to enter through the skin, and rarely, through the conjunctiva or placenta (194). Lesions in the throat and mouth then release virus into the saliva, and coughing, in turn, spreads virus in the air for subsequent transmission. VARV may be transmitted by saliva. Upon inhalation, the virus migrates rapidly to regional lymph nodes and the blood. The first round of viremia occurs on the $3^{\text {rd }}$ or $4^{\text {th }}$ day post-infection, with further dissemination of the virus to viscera such as spleen, bone marrow, and other lymph nodes. By the 8th day post-infection, the virus has undergone an exponential replication, when the secondary viremia occurs, followed by onset of fever and toxemia. VARV localizes in blood vessels in the skin (dermis) and the oropharyngeal mucosa. This leads to the appearance of a rash (enanthem and exanthem). By days 12-14 flu-like symptoms such as high fever, headache, and backache appear. Further rashes in the mouth and throat, and on the face, arms, trunk, and legs usually come next. The rashes then turn into deeply imbedded vesicular lesion pustules, which gradually become desiccated, leading to crusting or scabbing of the lesions. Infected individuals usually die due to overwhelming toxemia(85). Those who recover from the disease may be left with serious pathological conditions, including widespread skin scars, hearing and/or sight loss and in some cases, other organ damage $(267,673)$.

\subsubsection{Vaccination}

Variolation, a process adopted by practitioners in India, Africa and the Ottoman Empire in the early 1700 s, was the deliberate infection with smallpox, whereby dried VARV scabs were introduced into the nose of an individual to induce a mild form of the disease. Upon recovery, individuals were immune from smallpox. Variolation reduced 
the mortality rate significantly from $30 \%$ to $1 \%$ (747). In 1770 , Edward Jenner noted that milkmaids who had been exposed to CPXV did not develop VARV symptoms upon exposure, except for a few pox around the site of variolation. This observation led Jenner to 'vaccinate' (vacca meaning cow) a boy with CPXV, then variolate him with smallpox. Similar to what Jenner had observed with the milkmaid, the boy did not develop any smallpox lesions. Jenner's method of vaccination was widely used in Europe and America over the ensuing years, largely because vaccination had fewer side effects than variolation. 'Dryvax' was a freeze-dried calf lymph produced by American Home Products in the 1800s as a smallpox vaccine (1). During the 19th century, VACV gradually replaced CPXV virus for vaccination purposes. In 1959 , the $12^{\text {th }}$ World Health Assembly of the WHO passed the first resolution for global eradication of smallpox. But it was not until January 1, 1967, when the necessary resources were dedicated to the project that the WHO eradication program began. The WHO declared the global eradication of smallpox in December 1979 (194).

\subsection{VACV}

VACV is the most intensively studied prototypic member of the poxvirus family, due to its role as the vaccine agent used during the WHO campaign for eradication of smallpox. In addition, it has also been widely used as an expression vector for foreign genes and as a live recombinant vaccine for infectious diseases and cancer $(351,400$, $501,566)$. The specific origin of VACV is unknown. There are currently five suggested origins: 1) from VARV by passage in cows 2) from VARV by passage in humans via variolation 3) from hybridization between CPXV and VARV 4) from CPXV by passage 
in laboratory cultures 5) from CPXV by serial passage in animal skin $(64,191)$.

Different strains of VACV have been generated in different cities throughout Europe, Asia and America; e.g. Ankara (MVA) or Copenhagen (CPN) strains.

Like other members of the poxvirus family, VACV is a large double stranded DNA virus measuring some $360 \mathrm{~nm}$ in the largest dimension. Electron microscopy (EM) analysis reveals a brick shape for VACV virions (unlike the general rounded or helical morphology of many viruses), where a DNA core is associated with protein-containing lateral bodies and surrounded by either one or two viral membranes (453).

\subsubsection{VACV Replication cycle}

Like all poxviruses, VACV has an autonomous transcription system and replicates its DNA in the host cell cytoplasm. Therefore, the viral genome encodes many of the enzymes needed for the expression of early and intermediate viral genes and the construction of new viral progeny. Notably, the $192 \mathrm{~kb}$ DNA genome encodes approximately 200 proteins involved in diverse aspects of the viral life cycle (450). Early proteins are involved in viral DNA synthesis, the generation of nucleotide precursors, the enhancement of host cell growth and viral escape from the host immune response. In addition, virally-encoded proteins modify the host transcriptional and/or translational machinery to the advantage of the virus. These early genes are further divided into immediate and delayed early sub-classes (36). After DNA replication the intermediate and late genes are expressed. Intermediate genes mostly encode regulatory proteins that induce the transcription of late genes. The late genes encode components of the virion capsid as well as the factors needed to initiate the next round of replication (448). VACV 
particles are assembled within 6-8 h post-infection (p.i.) and mature in perinuclear cytoplasmic regions, so called "viral factories" in infected cells $(177,689)$. The first visible structures during the replication cycle in these viral factories are crescent-shaped lipids and proteins, which grow to form a structure that surrounds the virus core: the immature virion (IV). During the assembly and morphogenesis processes a number of cellular protein-modification pathways are utilized by the virus for its own protein maturation. This includes phosphorylation (570), acylation (201), myristylation (202), proteolytic processing (708) and ADP- ribosylation (122).

VACV produces two related but antigenically distinct infectious particles, namely intracellular mature virions (IMV) and extracellular enveloped virions (EEV)(30, 297, 679). While IMV constitute the majority of the infectious virions and remain inside the infected cell until cell lysis $(30,297)$, EEV virions are spontaneously released from infected cells. Unlike the EEV envelope, very little is known about the structure of IMV membranes. EM analysis suggested that the initial steps of VACV morphogenesis include the de novo formation of a single viral membrane $(278,656,657)$, however further immuno-EM study documented formation of a double viral cisternal membrane from the intermediate compartment between the ER and the Golgi $(242,643)$. Although the process of IMV membrane formation is complex and not well understood, it is certain that the basic configuration of IMV is simplified of a brick-shaped structure with an internal biconcave core, which is ornamented with spike-like structures wrapped into an outer membrane(s) $(145,299)$. Core proteins include the full complement of proteins necessary for activating early gene expression, structural proteins required for the assembly of the virion core, and virally encoded kinases and phosphatases (135). To date, 
$75 \mathrm{VACV}$-encoded, proteolytically processed proteins have been reported in the core of IMV, of which nine have been shown to play a role in VACV cell entry $(124,135)$. These include the gene products of A10L (p4a), A3L (p4b), L4R (p25K), F17R (p11), and A4L (p39) $(311,384,395,587)$. The membrane(s) surrounding the core contains three highly abundant proteins: A14L (16kDa), A17L (21kDa), and A13L (8kDa), three less abundant proteins: D8L/43 (32kDa), H3L/ $4(35 \mathrm{kDa})$, and a 27-kDa myristoylated protein, as well as a peripheral membrane protein, A27L $(14 \mathrm{kDa})^{*}(145,299,384)$.

The EEV contains an additional lipid membrane with at least 7 VACV-encoded polypeptides that are absent from IMV; six have been shown to be crucial for efficient envelope virus production $(637,638,705,706)$. The distinct biological and immunological properties of IMV and EEV arise from the different surface proteins that are adapted to their different roles in pathogenesis. Although EEV constitute only a small proportion of the viral progeny, they are responsible for the spread of infection in cell cultures. Moreover, it has been suggested that EEV have a role in VACV dissemination in infected mice, avoiding host antibody and complement, by virtue of being 'wrapped' in a host-derived membrane (511). During morphogenesis, a proportion of IMV are transported on microtubules within the cell and are surrounded by two intracellular membranes derived from the trans-Golgi network $(273,275,297,600)$ or elements of the endocytic pathway (endosomal cisternae) $(679,701,729)$, to form the intracellular enveloped virus (IEV). The mechanism for IEV formation is poorly understood, but at

\footnotetext{
* The identified membrane and core proteins of VACV virion are denoted by their corresponding open reading frames (ORF). The conventional designation of VACV ORF consists of a Hind III DNA fragment (A-O), followed by the number of the ORF in that fragment (numbered left to right), and by the direction of the ORF (L or R).
} 
least nine viral proteins are associated with the wrapping membranes: A27 $(559,729)$, B5 (183, 751), and (751), or F13 (73), A33 (566), A34(175), A36 (505), A56 (625), F12 (782), K2 $(89,169,720)$ and E2 (169). Among these proteins, F13, which is located on the cytoplasmic side of the wrapping membranes is palmitoylated on Cys 185 and 186, has a phospholipase-like function (it contains a highly conserved HKD motif, distinguishing for the PL superfamily (254), and mediates the formation of post-Golgi vesicles $(41,245,273,294,522)$. Mutations to the HKD motif or infection in the presence of butanol-1, a phospholipase inhibitor, have been shown to reduce IEV and EEV production (294). IEV particles are then transported to the host cell plasma membrane on microtubules (nocodazole reversibly inhibits the transportation), where they fuse with the cell membrane to form the cell-associated enveloped virus $(\mathrm{CEV})(112,214$, 277, 551, 732). Two VACV proteins, F12 and A36, have been implicated in IEV transport. Studies showed deletion of $F 12 L$ restricts CEV fusion at the host cell membrane after the formation of IEV particles $(277,701,782)$. In addition, deletion of $A 36$, affects IEV transport dramatically $(272,455,731,732)$. Of note, Herrero-Martinez et. al. showed that while the deletion of $A 36 R$ did not affect CEV formation profoundly, the deletion of $F 12 L$ had a significant effect on the CEV arrangement process (272). Fusion of IEV virions with the plasma membrane induces the polymerization of actin on the cytosolic side, forming so called "actin tails" to propel the CEV particle away from the cell either into adjacent cells or into the extracellular space $(277,702)$. During this process, A36 become phosphorylated by src kinases to induce virion dissociation from kinesin-1 and to trigger signaling cascades that in turn mediates actin tail formation beneath the $\operatorname{CEV}(205,475,702,753,754)$. Absence of $A 36 R$ or infection in the presence 
of Abl-family tyrosine kinase inhibitors have been shown to arrest initiation of actin tail formation, block CEV release (therefore smaller plaque size in vitro), reduce viral dissemination in vivo and protect mice against a lethal dose of infection $(417,505,548$, 592, 593). In addition to A36R, which encodes a 45-50 kDa protein, $\mathrm{p} 45-50$ (505), at least five other VACV genes are known to encode proteins involved in EEV membrane structure: F13L, encodes a $37 \mathrm{kDa}$ protein, p37(275); A34R, encodes a triplet of glycoproteins, gp22-24(175); A56R encodes the virus hemagglutinin (HA) gp86 (515, 625); A33R, encodes a 23-28 kDa glycoprotein, gp23-28(566); and B5R, encodes a 42 kDa glycoprotein, gp42(182, 300). CEV released from the host cell membrane by exocytosis or by direct IMV budding from the host plasma membrane (688) is termed EEV, which mediates long-range spread of VACV (15). The balance between the released $\mathrm{EEV}$ and the retained $\mathrm{CEV}$ virions on the host cell membrane could be affected by the strain of VACV $(510,512)$. Notably, a mutation in $A 34$ increases EEV release up to 50 times for the IHD-J strain of VACV compared to Western Reserve (WR) $(75,427)$. In addition, mutations in $B 5$ and $A 33$ increase EEV release $(271,319,388,417)$. The VACV replication cycle is summarized in Figure 1.4. 
Figure 1.4 Schematic overview of VACV life cycle.

(1) Post VACV entry to the host cell (see Figure 1.5), the virus loses its outer membrane(s) and releases its core into the cytoplasm (2) The core is transported on microtubules (3) Transcription of the early mRNAs leads to the core un-coating and DNA replication (4) Within the viral factory, immature virions (IV) are processed to form IMV (5) Some IMVs are transported to early endosomes and the trans-Golgi network for subsequent wrapping (6) IMV virions are wrapped to form IEVs which are then transported to the plasma membrane on microtubules (7) The outer IEV membrane fuses with the cell membrane, exposing CEV to cell surface. Actin tail formation occurs beneath the $\mathrm{CEV}$, promoting its release as an EEV. 


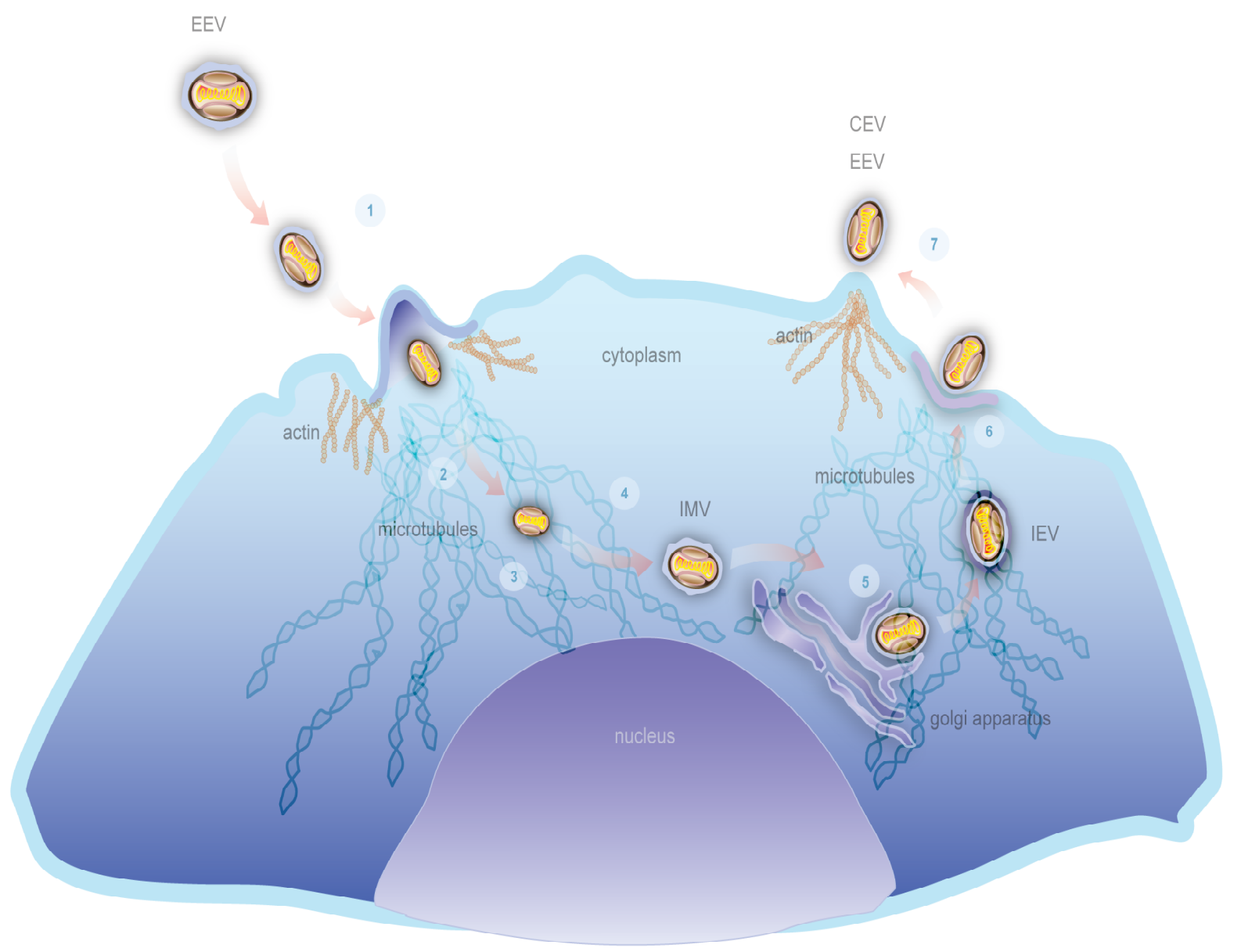




\subsubsection{VACV entry into the host cell}

Although the most critical steps in establishment of a viral infection is the ability of the virus to identify, bind and to penetrate into the host cell, these mechanisms remain largely undefined for poxviruses. The fact that VACV has a wide host range and is able to infect many different cells suggests that the cell surface receptors for the virus, if there are any, are ubiquitously expressed and highly conserved across species (125). Studies have suggested a role for lipid rafts, since cell membrane cholesterol depletion inhibits IMV entry (126). For the many viruses, receptor-mediated viral binding is crucial for efficient delivery of the viral genome into the cell. This may be mediated either by fusion of the viral membrane with the plasma membrane, or by fusion with the endosome membrane after endocytosis $(178,268)$. Viruses co-opt a wide range of receptors from different families of proteins, carbohydrates, or lipids, often in complex cell surface matrix structures (601). For instance, HIV binding to CD4 on T cells, and subsequent interactions with CCR5 or CXCR4 leads to membrane fusion and viral entry $(401,422$, 653). Poliovirus binds to an immunoglobulin-like protein, PVR, on the cell surface, leading to a rapid un-coating and delivery of viral particles into the cell $(230,317,432$, 531-533). Vesicular stomatitis virus (VSV) binds to $\alpha 2$-macroglobulin $(409,660)$, and the influenza virus hemagglutinin binds to the terminal carbohydrate side chain sialic acids on cells, the virus entering cells by endocytosis (749). Herpes simplex virus (HSV) binds to heparan sulfate side chains of GAGs and undergoes membrane fusion $(53,246$, 442).

There is much controversy surrounding VACV binding and entry into the host cell. Vanderplasschen \& Smith, have suggested that IMV and EEV bind to different cellular 
receptors (707) and enter cells by different mechanisms (705). However, other studies have indicated that a conserved viral fusion complex is necessary for the penetration of both viruses $(452,490,617,618,681,682)$. There is also evidence suggesting that IMVs bind to the plasma membrane and initiate its fusion to deliver viral cores into the host cell $(34,117,170,385,705)$ at neutral $\mathrm{pH}(117,170,308)$. IMV entry through fusion mechanisms is based on the double membrane hypothesis, since the remaining lipid bilayer would still be required to surround the core. (296). By contrast, a recent study provided evidence that IMV induces cell-surface blebbing, and that infection is dependent on the presence of exposed phosphatidylserine in the viral membrane, characteristic of apoptosis, to aid the macropinocytosis of virions into the cell (433). In addition, IMV entry requires actin mobilization and cell signaling that involves Rac, MEK, extracellular signal-regulated kinase, protein kinase $\mathrm{A}$, and protein kinase $\mathrm{C}$ activation (385). The study of EEV entry is somewhat restricted, due in part to the fragility of the EEV membrane and also the low abundance of EEV produced by most VACV strains. Two studies indicated that EEV enter host cells by endocytosis, and acidmediated disruption of the EEV membrane within intracellular vesicles $(296,705)$. Inhibition of the EEV entry by fluoride and cytochalasin B supports this mechanism (150, 514). Furthermore, acidic pH mediates VACV-induced syncytia formation $(170,231)$ and accelerates virus entry. During the syncytia formation (1) EEV viral particles accumulate at the cell membrane, (2) the EEV outer membrane is perturbed to form the IMV particle, (3) the IMV membrane binds to the plasma membrane leading to the insertion of the viral "entry complex" and (4) the viral "entry complex" fuses with the plasma membrane of an adjacent cell to form a syncytium (Figure 1.6)(385, 644). Cell-to-cell transmission of 
IMV by cell fusion is also mediated by syncytia formation (74). Viewed altogether, VACV entry can occur either at the plasma membrane or through a low-pH endosomal pathway, or by both mechanisms (Figure 1.5). 
Figure 1.5 Schematic representation of IMV and EEV entry.

The diagram illustrates the proposed models for IMV and EEV entry 
Figure 1.5
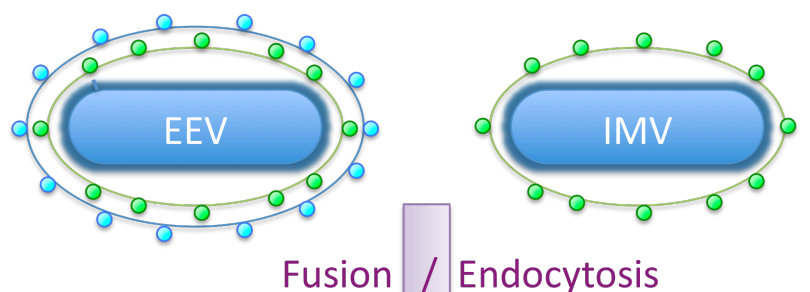

Fusion / Endocytosis

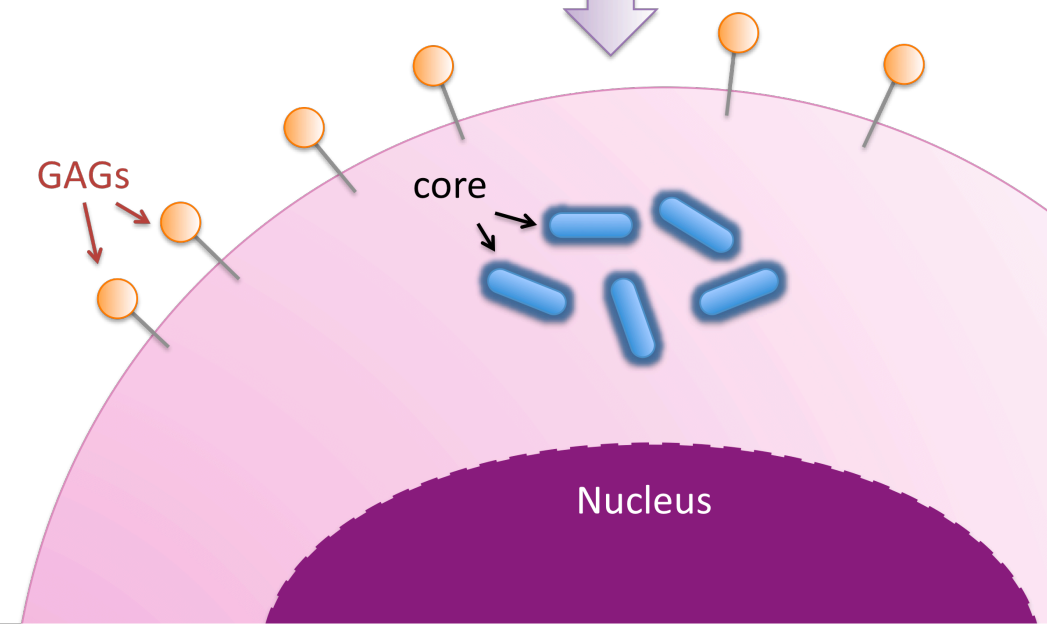


Figure 1.6 Schematic steps in formation of syncytia.

For visual simplicity, cell-cell fusion is represented as being induced by a single virion, while many virions are needed to form the actual syncytia. 
Figure 1.6
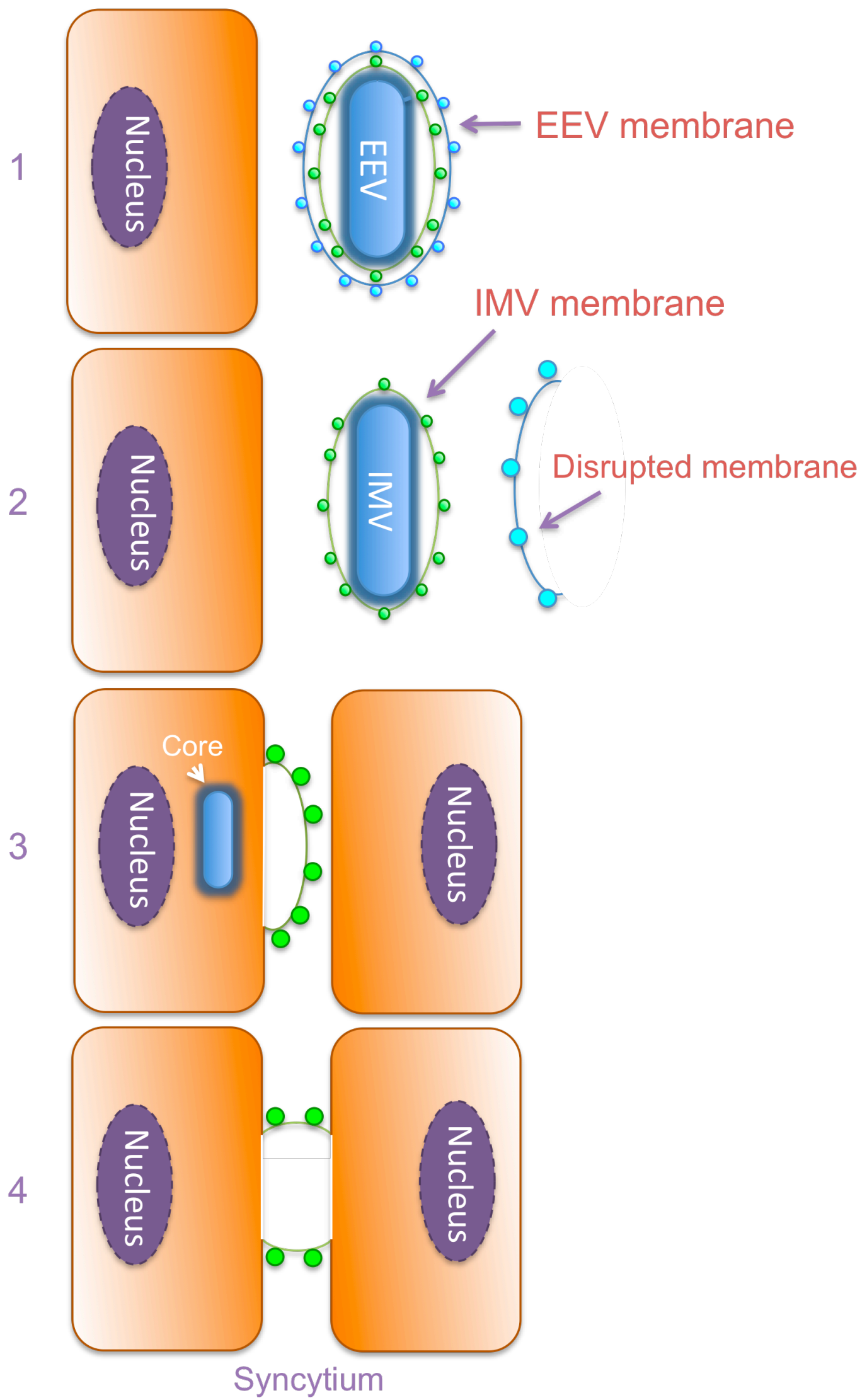
Among the 75 structural proteins that were characterized by proteomic analyses in $\operatorname{IMV}(124,135)$, more than 20 are membrane proteins, many of which are involved in viral morphogenesis (135). While at least 11 of these 20 membrane proteins have been identified as crucial for viral entry $(69,87,481,618)$, the others interact with the GAGs or laminins on the cell surface $(123,125,372)$. The membrane proteins A16L, A21L, A28L, H2R, and L5R all have a transmembrane domain, they are rich in cys residues, are required for cell-cell fusion, and have been shown to have critical roles in VACV entry into mammalian cells $(490,617,619,681,682)$. L1R is another IMV membrane protein, which suggested having a role in IMV entry, since a mAb recognizing L1R blocks IMV entry $(298,752)$. H3L and A27L are other membrane proteins that bind to heparan sulfate, D8L binds to chondroitin sulfate, whereas p4c interacts with laminins $(123,289$, 290, 372). However, at least individually, these proteins do not seem to be critical for viral entry, since the inhibitory effect of heparin on VACV entry has been shown to be incomplete and cell-type-dependent (111). The functions of six novel IMV membrane proteins G3L, C6L, E6R, A6L, A15L, J5L and A31R are still unknown (124, 618).

\subsubsection{WASp: A potential effector of vaccinia virus infection}

The ability of VACV to induce actin reorganization has led to the enumeration of the signaling cascade associated with actin polymerization, which is dependent on tyrosine phosphorylation events, the tyrosine kinase adaptor protein, Nck, and the Wiskott-Aldrich syndrome protein (WASp)(Figure 1.7) $(204,205)$. Notably, using gene expression microarrays to examine the gene signatures that accompany infection with

Western Reserve (WR) or modified Ankara (MAV) VACV strains, a set of human genes 
were identified, among which WASp is induced selectively during the course of VACV infection (248-250). Five members of the WASp family have been characterized: WASp, N-WASp, and three isoforms of WAVE (WASp family verprolin-homologous protein, also called Scar)(109). Members of the Rho family guanosine triphosphatase (GTPase) (Rac, Rho, and Cdc42) have also been implicated in actin cytoskeleton rearrangements (Figure 1-8A)(483, 549, 550). Activation of a tyrosine kinase-coupled receptor, e.g. the TCR, mediates the recruitment of WASp, SH3-containing proteins, Cdc42-GTP, and the Arp2/3 complex to the activation site. WASp is recruited via its proline-rich region in a Cdc42-independent fashion following receptor activation. Subsequent to interactions with $\mathrm{PIP}_{2}$ and active GTP-bound Cdc42, WASp is released from an auto-inhibitory state created by an interaction between the C-terminus VCA (Verproline homology, Cofilin homology, Acidic) domain and residues overlapping the GTPase-binding domain (GBD), following which the VCA binds to G-actin and activates actin nucleation through the Arp2/3 (a complex of seven evolutionarily conserved proteins (Figure 1-8A, B)(398, 462, 740). Cdc42-GTP interacts with the GBD of WASp/N-WASp, while $\mathrm{PIP}_{2}$ binds to a basic region located N-terminally to the $\operatorname{GBD}$ domain $(35,335,667)$. Additional structural domains in WASp/N-WASp interact with other members of the actin machinery. The N-terminal EVH1 homology domain binds to polyproline sequences in WIP (WASp Interacting Protein) (538). In addition, a central proline-rich domain (PRD) interacts with profilin and SH3 adaptor proteins, such as Grb2 and Nck, which in turn increases N-WASp-Arp2/3 complex-mediated actin assembly (Figure 1-8B)(107, 438, $552,563)$. Although IMV are capable of inducing the formation of actin tails that are similar to those seen in Listeria and Shigella infections, VACV is the only virus known to 
use actin-based motility to facilitate entry, intracellular translocation, egress and spreading between cells $(146,204)$. Notably, unlike Listeria and Shigella, a phosphotyrosine signal is required at the site of VACV actin-tail assembly. Furthermore, tyrosine phosphprylation of a host protein is required for the nucleation of new actin filaments by VACV and there is evidence that this phosphoprotein might be associated with cellular membranes that can nucleate actin filaments. VACV mediates actin assembly by the A36R protein $(205,567,579,753)$.

Frischknecht et. al. showed that phosphorylation of tyrosine 112 of A36R by Srcfamily kinases is necessary for the actin-based motility of VACV (205). Phosphorylated A36R, in turn interacts with the adaptor protein Nck, which leads to the recruitment of NWASp to the site of actin assembly $(205,420,438)$. In WASP knockout (KO) mice, Guerra et. al provided evidence that intranasal infection with VACV caused less weight loss and mortality compared to infection of wild-type (WT) mice. Dissemination of VACV into the ovaries was also reduced in the KO mice (248). In addition, VACV not only uses the microtubule cytoskeleton for its mobility but also increases microtubule dynamics through F11L, which binds to RhoA and blocks its ability to signal through the formin homology protein, mDia1 $(31,32,700)$. VACV infection has a potential role, therefore, in altering the cell cortex by blocking RhoA-mDia signaling, thereby targeting microtubules. This is likely to be a myosin-driven event, in the cell cortex prior to VACV egress (404). Viewed altogether, the data indicated that VACV co-opts actin polymerization signaling pathways for its efficient life cycle and subsequent spread of progeny. 


\section{Figure 1.7. Schematic representation of VACV-CCR5 signaling events}

Activation of the CCR5 receptor by virus leads to multiple tyrosine phosphorylation events culminating in viral replication and progeny. 
Figure 1.7

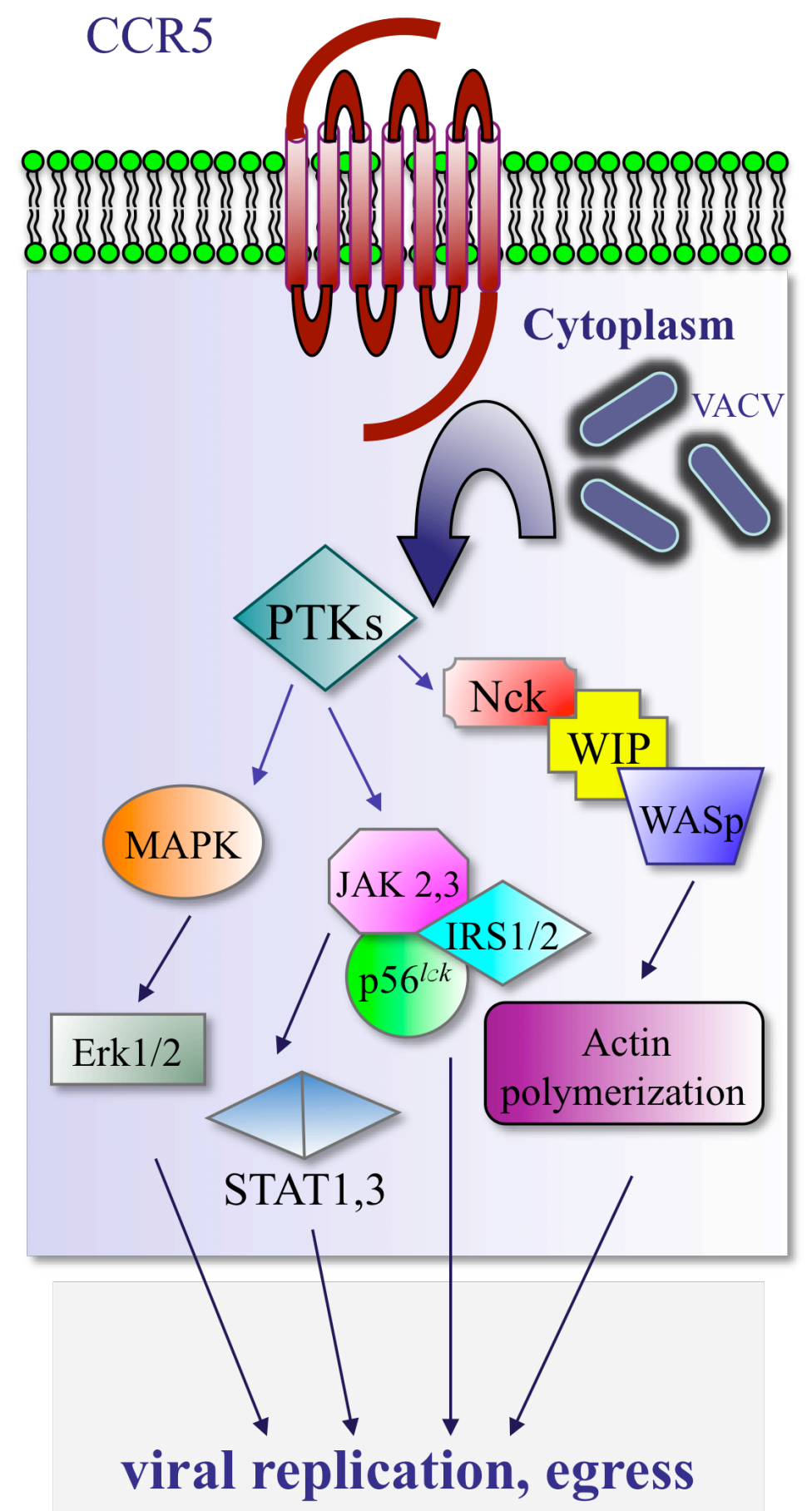


Figure 1.8. Schematic representation of WASp structure and signaling pathway A) Upon receptor activation, the auto-inhibited conformation of WASp is released by binding of Cdc42-GTP and PIP2 to GBD domain, allowing Arp2/3 complex activation. Binding of specific SH3 domain-containing adaptor proteins such as Nck and Grb2 can further stimulate actin polymerization. B) Domain structure of WASP. All WASP family members exhibit a very similar organization in their carboxyl terminus half with a proline-rich region (PRO) followed by a WH2 (also called V for verprolin-homology) domain, a central (C) region and an acidic (A) domain. 


\section{Figure 1.8}

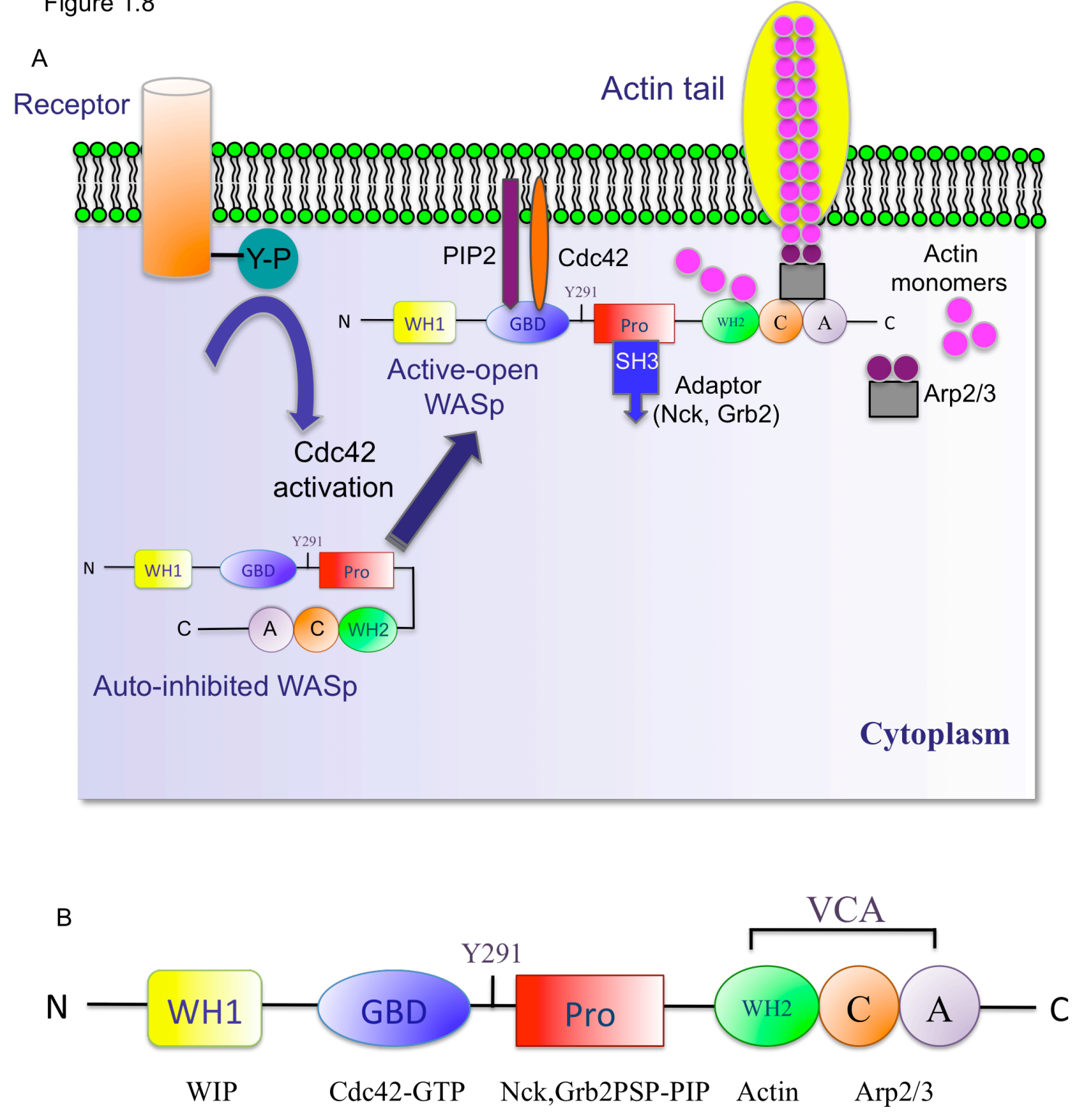




\subsubsection{VACV pathophysiology}

Smallpox vaccination is by either intradermal scarification or injection of VACV. After vaccination, VACV multiplies in the basal level of the epidermis, causing a local cellular reaction. In the next 4-5 days a circumscribed, solid elevation of skin appears as a result of viral replication. The fluid-filled lesion becomes pustular within 7-10 days and reaches a maximum size of $2-4 \mathrm{~cm}$ - the so called Jennerian pustule. This usually coincides with axillary lymphadenopathy and mild fever. Two to three weeks post vaccination, the pustule dries from the center, the scab sheds and forms a scar that usually remains throughout life as evidence of vaccination. Re-vaccination confers similar, yet accelerated symptoms $(85,93,238,258,280)$. No systemic viremia has been reported during administration of VACV in immunocompetent individuals for most strains of VACV (258), except for the NYCBOH ( New York City Board of Health) strain that has been reported to cause limited viremia in a small percentage of individuals during the pustule formation $(76,194)$. The host immune response to VACV infection is biphasic, with innate effectors - DCs, macrophages (119), natural killer (NK) cells $(91,100,519)$ - activated early, and antigen-specific T and B cell responses, later $(22,143,156,253)$. VACV activates innate immunity via both TLR-dependent and -independent pathways, which lead to the secretion of pro-inflammatory cytokines (mediated by TLR2 /MyD88) and interferon- $\beta$ (IFN- $\beta$ )(through a TLR-independent pathway) in vitro and in vivo (784). While the efficient induction of innate and adaptive immunity is dependent on TLR2/MyD88 signaling, TLR-independent production of IFN- $\beta$ has been shown to be critical for both innate and adaptive immunity (784). B-cell responses to VACV infection are evident based on the presence of VACV-specific circulating antibodies after 
vaccination. Children with agammaglobulinemia, with few exceptions (342), are fully protected from smallpox post vaccination, suggesting a more important role for $\mathrm{T}$ cell responses $(485,546)$. Furthermore, children exhibiting T- cell defects associated with thymic lymphopenia or Wiscott-Aldrich syndrome (WAS), develop major complications like giant cell pneumonia and encephalitis post VACV infection $(140,233,375)$. While a $\mathrm{CD} 8+\mathrm{T}$ cell response is essential for immunity and seems to clear primary VACV, $\mathrm{CD} 4^{+}$ $\mathrm{T}$ cells are thought to be involved in long-lasting protection against $\operatorname{VACV}(65,179)$. Notably, in $\mathrm{CD}^{-/-}$mice or in major histocompatibility complex class II-deficient mice, VACV clearance is delayed, $\mathrm{CD} 8^{+} \mathrm{T}$ cells exhibit proliferative and maturation defects and the mice fail to develop an antibody response to $\operatorname{VACV}(764,767)$.

Progressive vaccinia (also known as "vaccinia necrosum","vaccinia gangrenosa" and "disseminated vaccinia") is one of the rare yet most serious complications that occurs in infants, in older adults with defective cell-mediated immunity, and in individuals with compromised cell-mediated immunity or those receiving immunosuppressive medications (patients with AIDS, organ transplant recipients, individuals on cancer chemotherapy, on chronic corticosteroids, those with hematologic malignancies, and combined immunodeficiency disorders, or with hypogammaglobulinemia $(85,360,361$, $473,546)$. Progressive vaccinia is primarily characterized by the persistent enlargement of the vaccination pustule, followed by continuous replication of the virus leading to subsequent severe, necrotizing infection. In some cases, bacterial superinfection leading to death occurs after vaccination. CNS complications have also been reported in progressive vaccinia: encephalitis in children aged 6 months to 2 years, and demyelination, a less severe CNS reaction, in adults $(258,359,613)$. It is now widely 
believed that progressive vaccinia is the result of defective cellular immune functions rather than an inability to produce anti-VACV antibodies (207). Given the serious sequelae of progressive vaccinia, VACV should not be administered to children younger than 3 years, to patients with eczema or CNS disorders, or to individuals who are immunologically compromised.

\subsubsection{VACV-induced immunomodulation}

While it is indeed important to consider the entire life cycle of pathogens, it is their ability to neutralize the host immune responses as well as their successful attempt for long-term survival that defines them as pathogens. The battle between the pathogen and the host, not surprisingly, has provided a unique opportunity for both to evolve towards a mutual evolutionary change. Simply put, this "change of a biological object triggered by the change of a related object" is called co-evolutionary process (771). In host-pathogen case, co-evolution includes the evolution of a host species and its parasites. Viruses are not an exception and display a highly dynamic process to deal with the host defense system. In fact the footprints of the co-evolutionary process through exchanging of the functional molecules is evident from the presence of viral genomes, virus-like elements or sequence identities (i.e. between some plant and animal RNA viruses) in the genomes of differentiated organisms as well as shared functional motifs between cells and viruses, which suggest viruses as potential active agents of horizontal gene transfer $(54,98,658)$. The possible horizontal gene transfer gives the advantage to the viruses to acquire specific functions to target subsets of cells; this includes the ability gained by viruses to overcome the host defense system. Viruses utilize two strategies to overcome host defense system: interaction and evasion. Interaction includes expression of a number of 
genes, which their products contribute to evade the detection and destruction mechanism by the host immune response. Evasion which is mainly represented by RNA viruses utilizes genetic variation to escape the selective pressures intended to limit viral replication (54). The molecular basis of this adaptive strategy is the high rate of occurrence of mutations during RNA genome replication Some small DNA viruses, such as the papillomaviruses, and even some RNA viruses, such as hepatitis $\mathrm{C}$ virus, utilize both interaction and evasion strategies to survive $(301,302)$. Large complex DNA viruses, such as the poxviruses, express a number of genes, whose products have immunomodulatory specificity and are involved in evading the host immune response. These products include homologues of cytokines, chemokines and their receptors, and those involved in modulation of signal transduction, inhibition of apoptosis, downregulation of major histocompatibility complex (MHC) proteins, inhibition of cytotoxic T lymphocytes (CTLs) and/or natural killer (NK) cells activity $(9-11,13,737)$.

The linear double-stranded DNA genome of poxviruses is closed at both ends by single-stranded hairpin loops. Genes that are located in the central region of the genome are mostly conserved among the poxviruses and are likely to be involved in common functions such as replication or virion assembly, whereas those genes that lie at the ends of the genome are more variable among species and are generally involved in host range restriction or immune subversion (451). The inverted terminal repeat (ITR) is close to both ends, ranges in length from 58 bp in VARV (622) to 12,400 bp in Shope fibroma virus (746), and encodes from zero, in VARV $(102,415,622,624)$, to 12 ORFs in myxoma virus (102). Within each of the ITRs of the VACV genome the epidermal growth factor-like protein (vEGF) is encoded, which has been shown to mitogenically 
stimulate neighboring quiescent cells $(316,611,639,691)$. Adjacent to the ITRs at both ends are regions encoding the host range and immunomodulatory proteins. The immunomodulatory proteins encoded by poxviruses are:

\subsubsection{Virokines and Viroceptors;}

Poxviruses encode virokines and viroreceptors in their genomes to mimic hostderived molecules. Virokines are virus-encoded secreted proteins that mimic cytokines, complement regulators, or other humoral inhibitors. Viroceptors, are either secreted or localized to the surface of infected cells and are protein mimetics of cytokine/chemokine receptors, capable of binding and sequestering cytokines and/or chemokines.

\subsection{Proteins that block the complement pathways}

VACV complement control protein (VCP) is a protein with amino acid similarity to the family of complement control proteins, has four tandem copies of a short consensus repeat (SCR) and is a major virokine secreted from cells infected with VACV $(340,341) . \mathrm{C} 3 \mathrm{~L}$, a $35-\mathrm{kDa}$ secretory protein, is one such VCP that binds to complement factors $\mathrm{C} 3 \mathrm{~b}$ and $\mathrm{C} 4 \mathrm{~b}$ and thereby prevents the activation of complement by either the classical or alternative pathway $(339,341)$. VCP, therefore, is as anti-inflammatory agent that potentially could have therapeutic indication for preventing xeno-rejection by inhibiting complement and preventing cytotoxic cell-mediated death $(7,23)$. VCP is capable of binding to and interacting with GAGs $(468,640)$ through its heparin-binding domains within SCR 1 and $4(264,328,572,640)$. Binding to GAGs may not only enable VCP to localize to the cell surface and facilitate its uptake by mast cells and endothelial cells, but also might inhibit chemokine-mediated migration of leukocytes (148). VACV 
also encodes another protein, the gene product of B5R (229), which is a 42-kDa protein with four SCRs, is located on the outer envelope of the virus $(182,300,411,671)$ and exhibits amino acid similarity to complement control proteins. A role for this protein in complement inactivation (584) is required for IEV formation, actin polymerization, viral egress and the development of normal plaque size $(183,417,418,558,592,730,732$, 751). In other studies, IMV but not EEV, have been shown to be sensitive to complement inhibition: EEV harvested from rat cells expressing CD55 and CD59 (human regulators of complement) were more resistant to human complement compared to EEV collected from control rat cells (706). Since EEV's outer membrane technically engulfs the components of the host membrane proteins, these data might suggest that poxviruses may also avoid the complement system by preserving components of the host plasma membrane, such as regulators of complement on the EEV outer membrane.

\subsection{Proteins that block antigen presentation to the MHC}

Recognition of viral antigens by cytolytic T lymphocytes (CTL) is a critical step in an immune response to many viral infections. This occurs through the interaction between the T cell receptor (TCR) of a cytotoxic cell and a viral peptide presented on MHC-1 molecule on an antigen presenting cell (APC)(500). Although there are studies indicating that poxvirus-infected APCs fail to present certain class II-restricted epitopes $(168,203,444)$, it is the decreased expression of class I MHC molecules that correlates with the extent of systemic spread and viral replication during the course of infection. Poxviruses, including VACV $(90,142,523,680)$, have been shown to interfere with 
ectopic presentation of MHC-1 by targeting each step of its trafficking as well as by blocking its association with peptides (424). In fact, inhibition of TAP- (transporter associated with antigen processing-) dependent peptide translocation from the cytoplasm to the ER (366), arresting combined MHC-I molecule in the ER (5) or mediating MHC-I translocation to the cytosol and subsequent degradation by proteasomes (745), preventing the export of assembled molecules to the cell surface (5) as well as inhibiting proinflammatory cytokines (will be discussed later) such as TNF and IFN that regulate MHC expression, are among the inhibitory mechanisms that poxviruses utilize to interfere with MHC-1-dependant viral clearance. Although this inhibition seems to be selective for certain epitopes and can be overcome by enhancing Ag degradation, as well as alterations in Ag processing (680) it certainly has major effect on reducing the efficiency of the CMI (cell-mediated immunity) response. The extent of poxvirus-induced class I MHC downregulation varies among poxviruses. For instance, while infection with myxoma virus results in severe CMI suppression and a distinct MHC-1 downregulation (>90\%) (79), VACV does not impose comparable systemic immunosuppression and causes only moderate depletion of ectopic MHC-1 (611). Downregulation of MHC-1 not only protects the infected cells from CTL-mediated cytolysis, but also reduces the MHC-1dependent inhibitory signals required to for killing by NK cells. Interestingly, genes encoding MHC-1 homologs have been identified in a few poxviruses such as swinepox virus, which might suggest a mechanism for blocking the increased susceptibility to NK cells (4). Downregulation of ectopic presentation of CD4, required for MHC-II-mediated antigen-induced $\mathrm{T}$ cell activation, has also been shown post IMV infection (59). Early viral protein production seems to be involved in mediating the degradation of CD4 by 
lysosomes, which in turn targets the uncoupling of CD4-mediated signaling events at its intracellular association with p561ck, a member of the src family of protein tyrosine kinases (59).

\subsection{Proteins that block interferon signaling pathways}

IFNs-a/b are potent antiviral agents, also associated with cell-mediated Thl immune responses $(209,590,615)$. The production of IFNs can be induced by a variety of stimuli, such as virus infection or exposure to mitogens or dsRNA. Following their transcriptional activation, production and secretion from the cell, IFNs-a/b bind to specific cell surface receptors and induce signal transduction via JAK kinase activation and subsequent phosphorylation of specific members of STATs. Phosphorylatedactivated STATs form dimers that translocate to the nucleus and activate transcription of IFN-responsive genes (209). Poxviruses have anti-IFN strategies to disrupt components of IFN-dependent antiviral responses. These include inhibition of IFN induction, receptor mimicry that sequesters IFNs away from their cognate cell surface receptors, phosphatases that block tSTAT-mediated signaling (VACV H1L gene encodes a phosphatase that prevents IFN-induced activation of STAT-1(469), inhibition of IFNinduced gene transcription and subsequent blockade of protein mediators of the antiviral response, such as protein kinase PKR and 2'-5' oligoadenylates synthetase (OAS)(320, $472,590,611,615,733)$.

\subsection{Intracellular inhibitors of IFN}

Poxviruses are also capable of targeting intracellular signaling components of the IFN response, including PKR and 2',5'-OAS. Poxviral dsRNA produced during infection 
activates these enzymes, which leads to inhibition of translation and synthesis of viral

proteins in infected cells, and the subsequent induction of apoptosis $(55,219,306,590)$. VACV encodes two proteins to target the action of PKR and/or 2'-5' OAS within the cell. The first is a $25 \mathrm{kDa}$ protein product of gene $\mathrm{E} 3 \mathrm{~L}$, which, by virtue of its ability to bind dsRNA prevents PKR and 2'-5' OAS activation (118). This results in diminished phosphorylation of eIF2 $\alpha$ (eukaryotic initiation factor $2 \alpha$ ) (621), IRF-3, and IRF-7 (634). In addition, E3L has a role in reducing adenosine deaminase editing activity (381) as well as in the inhibition of IFN $\beta$ gene upregulation, mediated by blocking IRF-3 activation (765). The second protein is a $10.8 \mathrm{kDa}$ protein product of gene $\mathrm{K} 3 \mathrm{~L}$, with amino acid similarity to eIF2a, which functions as a pseudo-substrate for activated $\operatorname{PKR}(157,777)$. Deletion of either E3L or K3L genes renders VACV more sensitive to IFN, although the proteins function in different ways and loss of $\mathrm{K} 3 \mathrm{~L}$ has been shown to confer a host range phenotype $(62,63,84)$. Orthologs of E3L and K3L have also been reported for other members of the poxvirus family such as myxoma virus, Yaba-like disease virus, VARV, Shope fibroma virus, swinepox virus, and orf virus (58, 322, 425, 426, 746).

\subsection{Extracellular inhibitors of IFN}

In addition to encoding proteins that target effector molecules associated with an IFN response, poxviruses encode proteins that inhibit the action of IFN at the extracellular level. Specifically, soluble IFN receptors for either type I (IFNs-a/b) or type II (IFN-g) IFNs. Notably, by binding to circulating IFNs these soluble receptors prevent IFN binding to cell surface IFN receptors, thereby preventing an IFN antiviral response. 


\subsection{A soluble IFN- $\gamma$ receptor}

The first soluble IFN receptor identified, M-T7, was in myxoma virus and was shown to interact with IFN- $\gamma$ (699). All poxviruses encode soluble IFN- $\gamma$ receptors, however their specificity for IFN- $\gamma$ varies among the different species $(16,456,699)$. Unlike the mammalian receptors for IFN- $\gamma$ that are species-specific, the soluble IFN- $\gamma$ receptors encoded by most orthopoxviruses bind to different IFN- $\gamma$ s across a wide range of species (456). Although M-T7 has been shown to have a stable trimer conformation in solution (354), the soluble IFN- $\gamma$ receptors in VACV, camelpox virus, and CPXV exist naturally as homodimers (15). The IFN $\gamma$ receptor in VACV (vIFN- $\gamma \mathrm{R})$ is encoded by gene $\mathrm{B} 8 \mathrm{R}$ and is a $35 \mathrm{kDa}$ glycoprotein with amino acid similarity to the extracellular domain of the type II IFN receptor. Alcami et. al. showed that expression of this gene in insect cells infected with a recombinant baculovirus resulted in secretion of the protein that formed a complex with IFN- $\gamma$, inhibited IFN- $\gamma$ binding to the cells and blocked the antiviral activity of IFN- $\gamma(16)$. While there are conflicting data regarding the effects of deletion of the B8R gene on VACV infection in mice, in terms of virulence, in rabbits the absence of B8R resulted in a milder VACV infection $(652,666)$. Notably, the B8R gene product does not bind mouse IFN- $\gamma$ with high affinity, but does bind rabbit IFN- $\gamma$ $(16,456)$.

\subsection{Interferon- $\alpha / \beta$ binding proteins}

In addition to $\mathrm{B} 8 \mathrm{R}$, orthopoxviruses encode an $\mathrm{IFN}-\alpha / \beta$ receptor to evade the antiviral effects of type I IFNs. The best-characterized inhibitor of IFN $\alpha / \beta$ in poxviruses is the product of the B18R gene in the VACV strain Western Reserve (WR) and the 
B19R gene product in the Copenhagen strain $(132,665)$. Despite limited amino acid identity to the cellular IFN- $\alpha / \beta$ receptor, the B18R protein, with its three immunoglobulin- (Ig-) like domains, interacts with IFN- $\alpha / \beta$ and IFN- $\omega$ with high affinity $(376,665,703)$. Similar to vIFN- $\gamma$ R, B18R binds IFNs from different species, albeit with varying affinities $(16,456,665)$. The VACV B18R protein is expressed as two molecular species of 52 and 60 to $65 \mathrm{kDa}$, the latter a secreted glycoprotein with a signal peptide at the N-terminus, no hydrophobic transmembrane domain at the $\mathrm{C}$ - terminus and with limited sequence similarity to the $\alpha$ subunits (IFNAR1) of mouse, human, and bovine type I IFN receptors $(14,132,635)$. Deletion of the B18R gene attenuates VACV virulence for both intranasal and intracranial infection in mice, indicating the importance of the B18R as well as type I IFNs in viral pathogenesis $(132,665)$. In addition to the soluble $\mathrm{B} 18 \mathrm{R}$, antiserum raised against VACV proteins recognized a protein on the surface of virus-infected cells at early time points p.i. $(694,696)$. This protein is a membrane-bound B18R (695) that might be anchored in the plasma membrane through its N-terminus hydrophobic sequence (443). Interestingly, the soluble VACV B18R protein is capable of attaching to IFN that is bound to its cell surface receptor (132), but the role of membrane-bound B18R is unknown.

\subsection{Proteins that inhibit IFN- $\gamma$ induction by binding to IL-18}

IL-18 enhances T and NK cell maturation, cytokine production and cytotoxicities $(154,436,492,773)$ and increases FasL expression on NK cells and consequently FasFasL-mediated apoptosis $(155,687)$. In addition, IL-18 together with IL-2, induces IL-13 production by $\mathrm{T}$ and NK cells in mice and, in the presence of TCR activation, promotes 
Th1-mediated immune responses and induces T cell IL-4, IL-10, IL-13, IFN- $\gamma$

production $(283,284)$. Poxviruses secrete a soluble IL-18 binding protein (bp) product of the C12L gene, vIL-18bp, with sequence similarities to the immunoglobulin domain of the human IL-18bp, a natural antagonist that blocks IL-18 from binding to its receptor (6, $82,484)$. vIL-18bp inhibits signaling mediated by activation of the IL-18R (82), and block the activity of caspase -1 . The latter effect would, in turn, inhibit the production of IL-1ß and IL-18 from pro-IL-1ß and pro-IL-18, respectively, through a caspase-1dependent cleavage activity $(326)$. VACV, ectromelia virus, $\operatorname{CPXV~}(78,642)$ and molluscum contagiosum virus (MC54L)(766) posses functional vIL-18bps and a similar virus-encoded protein is also predicted in other poxviruses such as VARV(413), monkeypox virus (623), Yaba-like disease virus (365) and swinepox virus (4). A VACV in which the IL-18bp gene C12L is targeted, exhibits attenuated virulence (664), and deletion of the corresponding gene from ectromelia virus caused a local elevation of NK cell function following intraperitoneal infection of mice (78).

\subsection{Soluble proteins that inhibit tumor necrosis factor (vTNFr)}

Tumor necrosis factor (TNF) is a multifunctional pro-inflammatory cytokine secreted predominantly by monocytes/macrophages and activated $\mathrm{T}$ cells, also a critical effector in apoptosis. Poxviruses target TNF by secreting a soluble TNFR, vTNFRs, which has sequence similarity to the extracellular domain (with no C-terminus transmembrane domain) of the TNF cell receptor counterpart, and forms functional oligomers that bind TNF prior to cellular TNFR engagement $(60,147,768)$. The bestcharacterized poxviral vTNFRs are the T2-like vTNFRs of leporipoxviruses (632) and the cytokine response modifier (Crm)-like TNFRs encoded by orthopoxviruses (147). 
In myxoma virus, the T2-like genes encode early, glycosylated proteins that interact with rabbit TNF with similar affinity to that of the rabbit cell surface TNFR $(632,698)$. Myxoma T2 (M-T2) inhibits the apoptosis of virus-infected lymphocytes $(397,602)$. The first two N-terminal cys-rich domains (CRD) seem to be involved in the anti-apoptotic properties of M-T2, while the ability of M-T2 to inhibit TNF has been attributed to the first three CRDs (609).

A family of Crm-like vTNFRs, including CrmB, CrmC, CrmD (147), CrmE (595), and newly recognized fifth member from CPXV (with sequence similarities to CD30)(502), has been characterized in various orthopoxviruses. CrmA is a distinct intracellular serpin-like modulator and acts as an inhibitor of the pro-inflammatory IL-1 $\beta$ converting enzyme (caspase-1) (542). In ectromelia virus, for which no functional CrmE genes have been identified (641), a related soluble CD30 homolog seems to block IFN and TNF production and inhibit inflammatory responses mediated by Th1, but not Th2 T cells (596). Depending on the viral strain, the activity and distribution of vTNFR-like proteins differs among orthopoxviruses. For instance, while most CPXV strains lack $\mathrm{CrmD}$, the gene is usually found in orthopoxviruses that are deficient for $\mathrm{CrmB}$ and CrmC $(12,147)$. In CPXV and VACV strain USSR, functional CrmEs have been characterized that are capable of binding to TNF from several species, but are only able to inhibit human TNF-mediated cytolysis $(543,595)$. Notably, while a few VACV strains have been shown to encode non-functional TNFR homologs, such as A53R (CrmC) and B28R (CrmB) (285), Lister, USSR, and Evans strains of VACV encode both soluble and cell-associated TNF-binding activities as a functional A53R (CrmC) gene product in the Lister and USSR strains and a CrmE-like protein in the USSR strain $(12,543)$. In addition 
to directly targeting TNF, some poxviruses utilize an alternative strategy to abolish TNFmediated intracellular signaling. The molluscum contagiosum FLICE inhibitory protein (vFLIP) product of the MC159L gene (220) and the CPV016 gene product in CPXV (489), block the activation of NF- $\mathrm{B}$, a crucial signaling effector in the TNF cascade.

\subsection{Intra- and extra-cellular Inhibition of IL-1}

Poxviruses also encode proteins that interrupt intracellular signaling mediated by IL-1 receptor activation. The VACV A46R and A52R proteins contain Toll-like/IL-1 receptor domains that are capable of disrupting IL-1 signaling and inhibit IL-1-induced NFא-B activation (82). In addition, VACV A52R functions as a dominant-negative, mimetic/truncated version of MyD88, an intermediary in IL-1, TLR4, and IL-18 signaling (82). Notably, VACV B15R and CPXV B14R encode secreted proteins that bind to IL-1 $\beta$, but not IL- $1 \alpha$ or the host IL-1 receptor antagonist $(14,641,650)$, thereby blocking IL-1 receptor mediated signal transduction. A B15R null VACV exhibited decreased virulence in mice infected via the intracranial route $(650)$, however increased virulence was observed when the mice were challenged with this virus intranasally (14). This may suggest that different viral proteins might be involved in VACV virulence during different routes of infection. In addition, E191, an IL-1R homolog encoded by ectromelia virus, binds to IL-1 $\beta$ and therefore prevent signaling downstream of the IL-1 receptor (641).

\subsection{Modulation of Chemokines}

In other studies, Alcami et. al. have identified a $35 \mathrm{kDa}$ soluble VACV protein present in some, but not all, VACV strains (17). This protein, vCKBP, interacts with 
CCL5. (17). There is accumulating evidence that poxviruses employ a variety of methods to modulate chemokine activity. These include chemokine binding proteins (CBP), homologs of chemokine receptors, and chemokine ligand mimics $(353,466,612)$. CBPs are classified in two sub-classes: low affinity (type I) and high affinity (type II) vCBPs. The myxoma virus IFN- $\gamma$ receptor homolog, M-T7, is the only member of the type IvCBPs, which binds to IFN $\gamma$ and a wide range of $\mathrm{CXC}, \mathrm{CC}$, and $\mathrm{C}$-chemokines, with no species specificity (354), and its interaction with GAGs disrupts the chemokine gradients $(356,612)$. Type II vCBPs (also termed CBP-IIs or vCCIs) lack amino acid identity with all known mammalian proteins (612) and specifically bind to CC-chemokines (but not C, CXC, or $\left.\mathrm{CX}_{3} \mathrm{C}\right)$ with high affinity and inhibit their binding to $\operatorname{GPCRs}(17,240,633$, 641). VACV CBP-II is capable of interacting with most, but not all, CC-chemokines, suggesting a level of selectivity (97). Of note, VACV 35-kDa CBP-II interacts with the GPCR binding site of CCL2 $(68,98,604)$ and not the heparin-binding domain (412). The myxoma virus CBP-II M-T1 protein interacts with GAGs and CC-chemokines, thereby mediating inhibition of chemokine activity at the site of infection (610). Chemokine ligand mimics have been identified for only two poxviruses: molluscum contagiosum virus and fowlpox virus $(3,616)$. The molluscum contagiosum virus chemokine, MC148R, is a secreted protein homolog to IL-11 receptor -locus chemokine (303), that was originally shown to inhibit leukocyte responses to a number of CC and CXC chemokines (153), and later shown to be a selective antagonist against human CCR8 (394), also capable of preventing allograft rejection in transgenic mice (162).

\subsection{Poxviral homologs of other host proteins}

Except for molluscum contagiosum and swinepox viruses most poxviruses also 
express a family of growth factor homologs and therefore can manipulate the signaling machinery connected to growth factor receptors to affect cell cycle regulation, proliferation and/or angiogenesis (208). An epidermal growth factor (EGF) homolog and a vascular endothelial growth factor (VEGF) homolog are two examples of viral proteins with an impact on virulence and virus dissemination. Semaphorin family homologs have also been characterized in Poxviridae $(133,336)$. VACV A39R is a $50-55-\mathrm{kDa}$ protein with a significant identity to cellular semaphorins $(133,213)$. Notably, semaphorin family homologs that resemble the extracellular region of SemaA7A have been identified not only in 8 of 15 VACV strains (213), but also in ectromelia virus and CPXV $(133,213)$.

\subsubsection{Poxviral inhibition of apoptosis}

Poxviruses block apoptosis by expressing specific proteins at early stages of virus replication $(521,580,626)$. These proteins may either be secreted to neutralize extracellular factors, e.g. TNF decoy proteins, or can function as either caspase activation inhibitors or suicide caspase substrates $(521,626,627)$. To inhibit apoptosis Molluscum contagiosum virus encodes MC159L and MC160L proteins, related to cellular death effector domain (DED)-containing proteins that bind to both caspase-8 and the Fasassociated death domain adaptor molecules $(627,678)$. It has been suggested that they simultaneously block the recruitment of pro-caspase- 8 to death receptor complexes at the plasma membrane and block the apoptotic cascade (626). Poxviruses also express intracellular serpins with anti-apoptotic characteristics. The CPXV serpin, CrmA, is an anti-apoptotic protein that functions to target both intrinsic and extrinsic death pathways (542). It potently blocks caspase-8- and caspase-1-dependent apoptosis pathways in vitro and in vivo $(345,581)$, without interfering with caspase-9-dependent death, at least in 
vivo (583). The finding that CrmA can block Fas-induced apoptosis (677) and inhibit the serine protease granzyme $\mathrm{B}$, suggests that $\mathrm{CrmA}$ is capable of protecting cells from CTL/NK cell-induced perforin-dependent apoptosis (530). Moreover, CrmA is the prototypical poxvirus caspase suicide substrate, blocking the activity of caspase- 8 and caspase-10 and inhibiting death pathways caused by cell injury or stimuli such as growth factor deprivation, hypoxia, detachment from the extracellular matrix, or TNF and Fas ligation $(80,251,676)$. Not as effective as CrmA, some of the SPI-2 family of poxvirus serpins have also been shown to inhibit apoptosis. Although the VACV SPI-2 protein product of B13R gene protects cells from Fas- and TNF-dependent death (167), targeted disruption of SPI-2 has limited effects on virulence (326). By contrast, MV SERP-2 blocks both caspase-1 and granzyme B in infected lymphoid cells to inhibit cell death, and its targeted disruption affects viral virulence significantly $(434,692)$. 


\subsection{Hypothesis and Objectives}

Hypothesis: VACV-inducible intracellular signaling events mediated by CCR5 activation have immuno-pathogenic consequences in vitro and in vivo.

\section{Objectives:}

Chapter 2: Examine the contribution of CCR5 mediated tyrosine phosphorylation signaling to cellular permissivity to VACV infection.

Chapter 3: Examine the role of CCR5 in dissemination of VACV in an intranasal mouse model of infection.

Chapter 4: Evaluate the role of glomulin in cMet-mediated VACV infection in vitro. 


\title{
Chapter 2
}

\section{Vaccinia virus activation of CCR5 invokes tyrosine phosphorylation signaling events that support virus replication}

\author{
Chapter 2 was published as \\ Rahbar R., Murooka T., Hinek A., Galligan C., Sassano A., Yu C., Srivastava K., \\ Platanias L. and Fish E.N. Vaccinia virus activation of CCR5 invokes tyrosine \\ phosphorylation signaling events that support virus replication.
}

J Virol. 2006 Jul;80(14):7245-59.

R.R. performed all experiments (except for Fig $1 \mathrm{bII}$ and 1bIII), analyzed the data and drafted the manuscript. 


\subsection{ABSTRACT}

Vaccinia virus, a poxvirus, produces structurally distinct forms of virions for which the immediate events following cell entry are ill-defined. We provide evidence that intracellular mature virions (IMV) enter both permissive and non-permissive T cell lines, and that introduction of CCR5 into non-permissive cells renders them permissive for vaccinia replication. Notably, cells expressing CCR5 in which tyrosine 339 in the intracellular region is substituted with phenylalanine, no longer support virus replication or virus-inducible activation of specific host cell signaling effectors: IRS-1, Grb2, Erk1/2. We show that following IMV entry into the cell, the intact but not the tyrosine deficient CCR5 is rapidly internalized and co-localizes with virus. This co-localization precedes virus-inducible signaling and replication.

\subsection{INTRODUCTION}

Vaccinia virus (VACV) is the most intensively studied member of the poxvirus family, related to its role as the vaccine agent responsible for the eradication of smallpox. Like other poxviruses, vaccinia is a large DNA virus that has an autonomous transcription system and replicates its DNA in the host cell cytoplasm, largely independent of the nucleus, although evidence suggests that the virus utilizes host cell proteins for transcription of intermediate and late genes $(88,136,447)$. VACV produces several distinct forms of viral particles that include the intracellular mature virus (IMV), the intracellular enveloped virus (IEV), the cell-associated enveloped virus (CEV) and the extracellular enveloped virus (EEV)(81, 514, 638). The vaccinia replication cycle takes place entirely in the host cell cytoplasm and 
comprises a sequence of discrete steps that begin with the entry of the viral particles and culminate with morphogenesis, release and egress. Although the exact mode of VACV binding and entry into cells is unknown and is complicated by the existence of low-affinity cell surface interactions with glycosaminoglycans (GAGs) that are celltype specific $(111,372,385,705)$, evidence for conserved membrane proteins, A28 and $\mathrm{H} 2$, determining poxvirus cell penetration does suggest a mechanism for cell entry, at least in cell lines (620). Until recently, no cell surface molecules on primary cells or cell lines have been demonstrated to be essential for poxvirus infection. However, new evidence was provided for the inducible expression of a receptor on primary $\mathrm{T}$ cells determining VACV binding and entry (7). Notably, activated T cells, but not resting $\mathrm{T}$ cells, express this uncharacterized virus receptor.

Originally viewed as inflammatory mediators, it is becoming increasingly apparent that chemokines interact with their cognate serpentine G-protein-coupled receptors to activate many diverse developmental and immunological operations, including viral clearance. Not surprisingly, therefore, viruses have evolved immune evasion strategies that subvert the chemokine system. HIV and SIV use CCR5 and CXCR4 as coreceptors to mediate their entry (630), while RSV employs the fractalkine receptor $\left(\mathrm{CX}_{3} \mathrm{CR} 1\right)$ to initiate infection (684). Poxviruses employ strategies to modulate chemokine activity, including virus-encoded chemokine-binding proteins, receptor homologs and ligand mimics $(353,466,612)$. Interestingly, the potential for the involvement of certain chemokine receptors in poxviral infection was suggested in studies utilizing the rabbit poxvirus, myxoma. Specifically, CCR5 was implicated in mediating cell target susceptibility to infection in BGMK cells (355), later shown to 
correlate with intracellular signaling(314). Myxoma virus infection of mouse fibroblasts elicits downstream signaling events that are pertussis toxin-insensitive and that may utilize CCR5, starting with tyrosine phosphorylation of the receptor itself and including activation of the Jak-Stat pathway, as well as the recruitment of p56 ${ }^{\text {lck }}$, and the IRS proteins (416). Certainly, there is considerable evidence for poxvirus activation of signaling effectors required to support viral replication $(24,160,314$, 597). The mechanism(s) whereby poxviruses activate these signaling effectors is unknown, and the notion that intracellular virus or viral proteins may activate transmembrane receptors to initiate these signaling events, prompted the studies described herein. Accordingly, we undertook to investigate whether modulating CCR5 expression and activation influences the permissive phenotype in the context of infecting vaccinia IMV particles. 


\subsection{RESULTS}

\subsubsection{Ectopic expression of CCR5 confers a permissive phenotype for vaccinia virus replication}

We have previously shown that NIH 3T3.CD4.CCR5 cells are permissive for myxoma virus infection (416). Evidence has been provided that the serine-threonine kinase, PAK-1, plays an important role in myxoma virus infection of $3 \mathrm{~T} 3$ cells, specifically distinguishing permissive NIH3T3.CD4.CCR5 from non-permissive NIH3T3.CD4 neo cells (314). In a first series of experiments we infected NIH3T3.CD4.CCR5 with a different poxvirus, VACV that expresses the E.coli ßgalactosidase gene under the control of a late viral promoter, and identified viral infection (Figure 1). To investigate the contribution of cell surface expression of CCR5 to viral infectivity, we performed studies in which the expression of CCR5 in NIH3T3.CD4.CCR5 cells was blocked, using CCR5-specific siRNA duplexes. Transfection of CCR5-specific RNA duplexes, but not scrambled siRNA, in NIH3T3.CD4.CCR5 cells resulted in significant downregulation of cell surface expressed CCR5 at 48hrs (Figure 2.1a). Notably, VACV infection of cells in which CCR5 expression was knocked down showed a dramatic decrease in viral infection (Figure 2.1 b, c). In parallel, we introduced CCR5 into the NIH3T3.CD4.neo cells by transfection, to investigate the contribution of CCR5 expression to their permissive phenotype. NIH3T3.CD4.neo cells are not permissive for VACV infection. Whereas transfection of vector alone had no effect, cell surface expression of CCR5 in the NIH3T3.CD4.neo cells rendered them permissive for VACV infection (Figure 2.2). 


\section{Figure 2.1}

\section{Knockdown of CCR5 in NIH3T3.CD4.CCR5 cells reduces vaccinia virus infection}

(a) NIH3T3.CD4.CCR5 cells were left untreated, or transfected with either CCR5specific siRNA or control, scrambled siRNA. At 24 (grey solid line) and 48 (dotted line) hrs post-transfection, cells surface CCR5 expression was determined by flow cytometric analysis using anti-CCR5 specific antibodies. Filled (negative) cytograms correspond to IgG reagents alone.

(b) NIH3T3.CD4.CCR5 cells were either left untreated (i) or infected with VACV at multiplicities of infection (m.o.i.) of 1 (ii, iv) or 10 (iii). 24 (i,ii,iii) or 48 (iv) hrs post infection, the monolayers were fixed and stained with X-gal to determine lacZ expression. (c) NIH3T3.CD4.CCR5 were transfected with either CCR5-specific siRNA (v) or control, scrambled siRNA (vi). $48 \mathrm{hrs}$ post transfection, monolayer cultures were infected with VACV at a m.o.i of 10. 24 hrs post infection, the monolayers were fixed and stained with X-gal to determine lacZ expression.

(d) NIH3T3.CD4.CCR5 infected cells from panel B were also analyzed for ßgalactosidase activity of cell lysates by means of the colorimetric assay (see Materials and Methods).

- NIH3T3.CD4.CCR5 cells, VACV infected cells; $\square$ NIH3T3.CD4.CCR5 cells transfected with control, scrambled siRNA, then VACV infected; $\square$ NIH3T3.CD4.CCR5 cells transfected with CCR5-specific siRNA, VACV infected; $\mathbf{W}$ uninfected NIH3T3.CD4.CCR5 cells. The mean +/- S.E. values of quadruplicate assays, representative of 2 independent experiments are shown. 
Figure 1

a
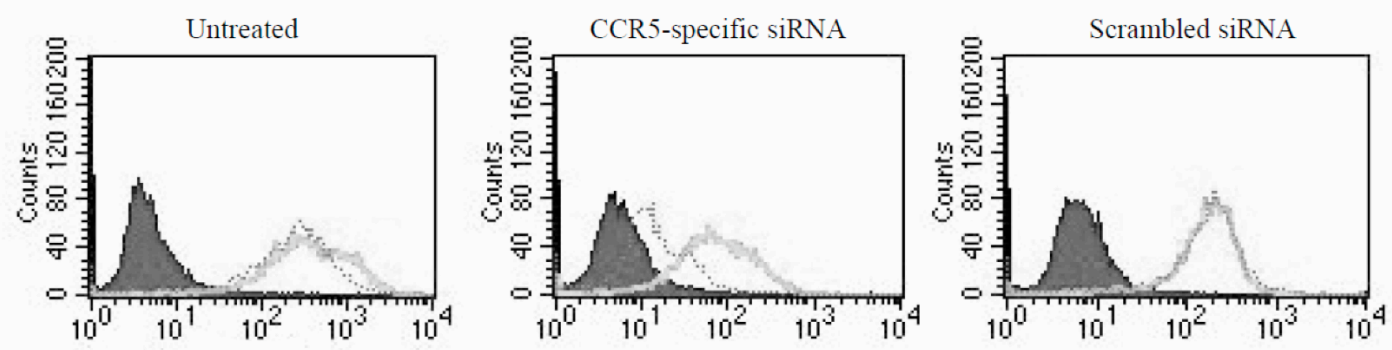

Fluorescence Intensity

b

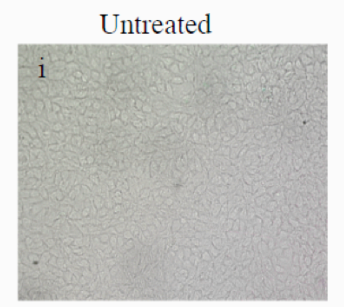

VACV m.o.i 1 (24 hrs.)
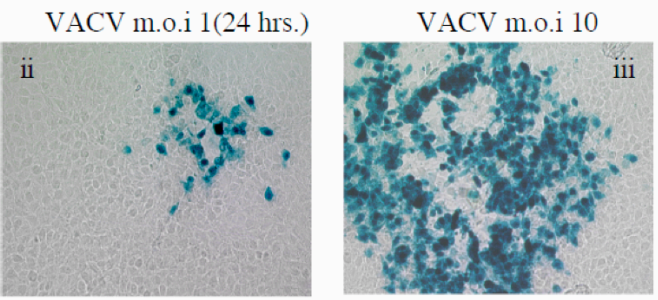

VACV m.o.i 1 (48 hrs.)

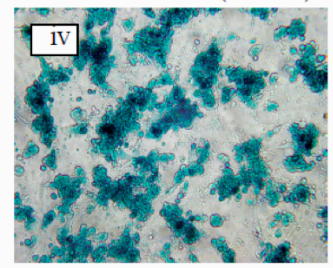

c

CCR5-specific siRNA + VACV m.o.i 10

scrambled siRNA

+ VACV m.o.i 10
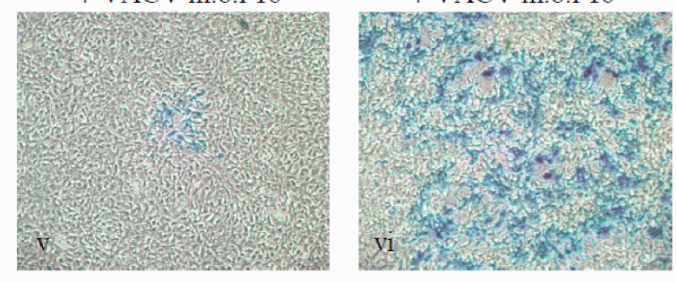

d

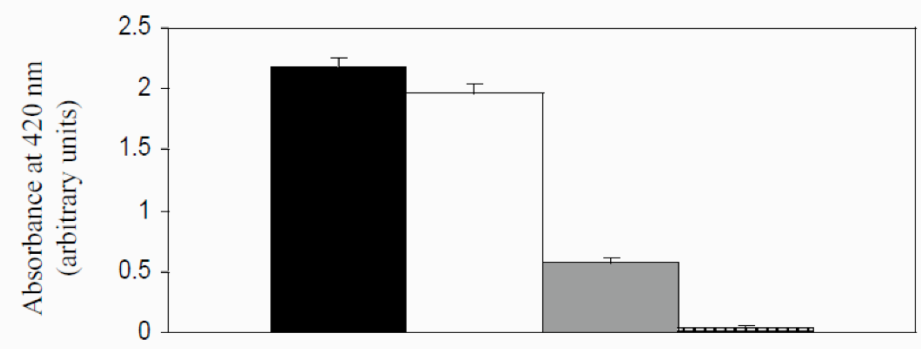




\section{Figure 2.2}

\section{Introduction of CCR5 into NIH3T3.CD4.neo cells renders them permissive for vaccinia virus infection}

CCR5 cDNA, CCR5Y307F, CCR5Y339F cDNA, or vector alone was introduced by transfection into NIH3T3.CD4.neo cells and cell surface CCR5 or mutant CCR5 expression determined by flow cytometry using anti-CCR5 specific antibodies that do not distinguish intact or mutant CCR5s (grey solid line). Filled (negative) cytograms correspond to IgG reagents alone. (a). Cells were infected with VACV at an m.o.i of 10. Cells were either fixed and X-gal staining performed (b), or LacZ activity was measured $16 \mathrm{hr}$ post infection using the $\beta$-galactosidase colorimetric assay (c). The mean +/- S.E. values of quadruplicate assays are shown, representative of 2 independent experiments. 
a
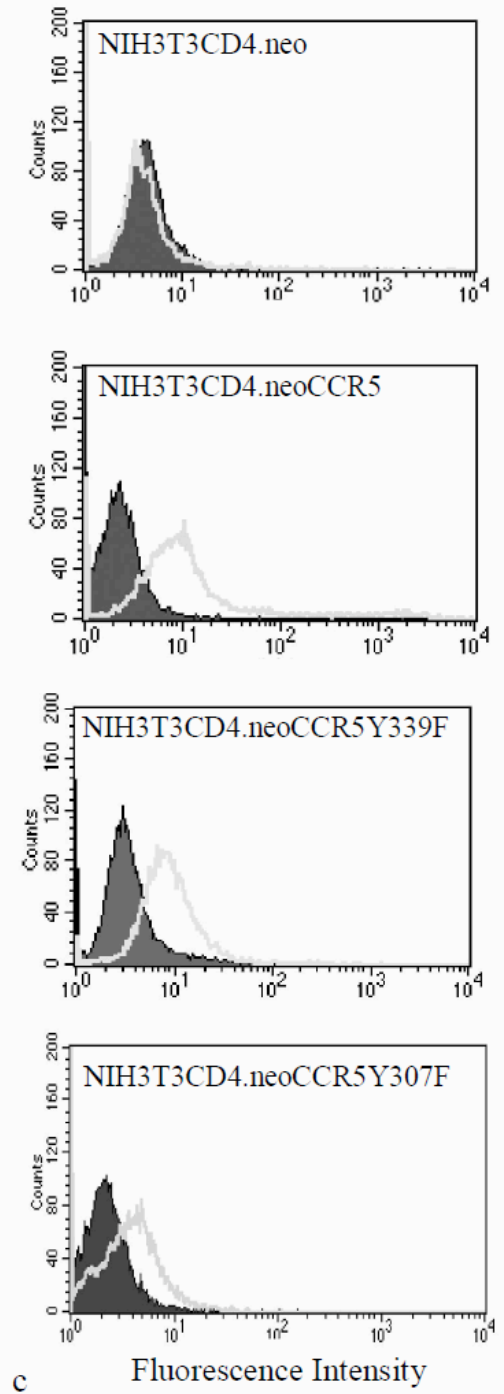

Figure 2
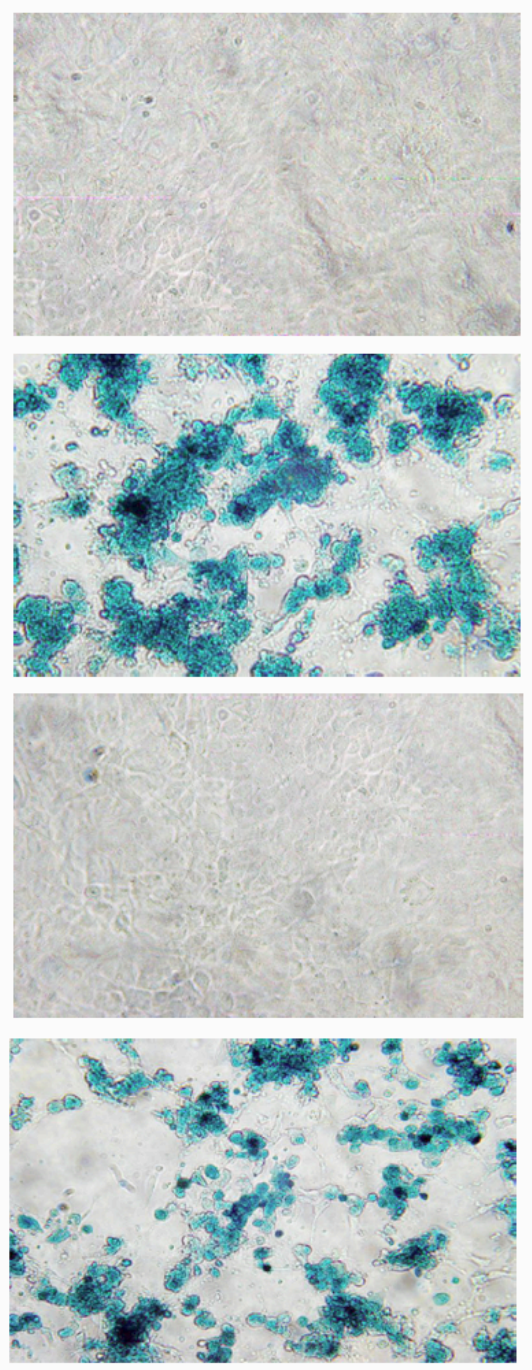
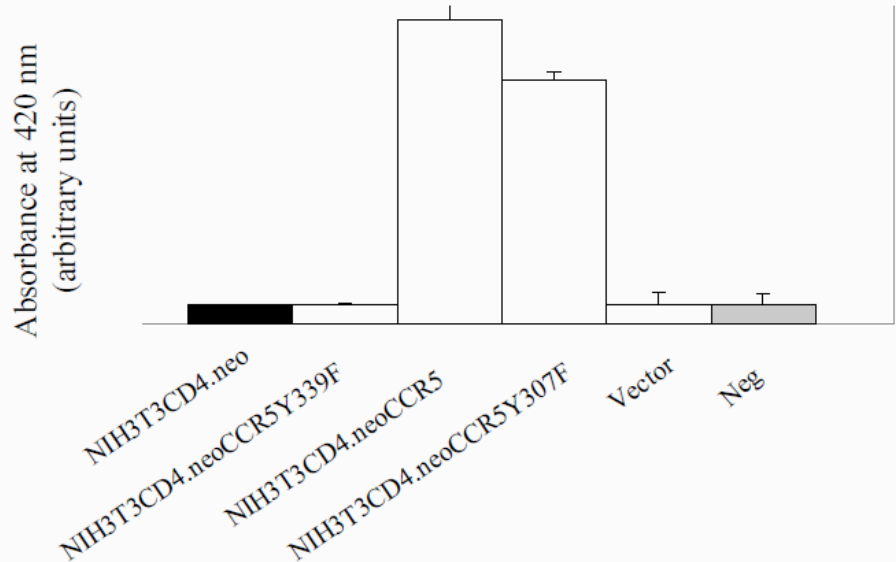
In an earlier study we provided evidence for myxoma virus-inducible CCR5 tyrosine phosphorylation and activation of tyrosine kinase signal transduction associated with a permissive phenotype (416). Tyr (Y)-127 lies in the second intracellular loop of the receptor in the DRY motif, highly conserved among CC chemokine receptors and implicated in mediating chemokine receptor signal transduction. For CCR2, the homologous tyrosine residue of the DRY motif (Y139) is the primary target for Jak2 mediated phosphorylation, and in the context of the CCR2b/CCL2 interaction, Jak 2 is required for G protein coupling. Mutating Y139 of CCR2b to a phenylalanine (F) results in an inactive receptor (CCR2bY139F), unable to recruit Jak2 and incapable of G protein activation (560). This mutant receptor confers a loss of function dominant-negative phenotype, and dimerizes to form non-functional complexes with CCR $2 b$ partners containing the functional tyrosine (562). In the context of CCR5, mutation of the DRY motif was shown to result in a non-functional receptor with a somewhat reduced surface expression, incapable of $\mathrm{G} \alpha$ subunit binding and signaling $(295,710)$. The other two intracellular tyrosine residues of CCR5, Y307 and Y339, reside in the C-terminal tail of the receptor. While Y307 is conserved among CC chemokine receptors, Y339 is unique to CCR5 and CCR4.

In the context of VACV infection, NIH3T3.CD4.neo cells expressing CCR5 in which all 3 intracellular tyrosine residues $(127,309,339)$ are substituted with phenylalanine (CCR5.YD3F), or CCR5 in which tyrosine 339 is replaced by phenylalanine (CCR5.Y339F), are non-permissive for viral infection, whereas cells expressing the CCR5.Y307F are permissive (Figure 2.2). 
Viewed altogether, these data suggest that in cells for which CCR5 will confer a permissive phenotype for infection, tyrosine 339 is critical.

Different leukocyte cell populations are variably permissive for VACV infection, T lymphocytes exhibiting poor infectivity (591). Accordingly, we examined the effect of ectopic CCR5 expression on VACV replication in primary T cells derived from human peripheral blood lymphocytes and in a human T cell line, PM1. In contrast to nonpermissive naïve T cells and parental PM1 cells, which both lack CCR5 expression; ectopic expression of intact CCR5 confers permissiveness for VACV infection, whereas ectopic expression of CCR5Y $33 \mathrm{~F}$ or CCR5Y339F fails to affect permissiveness (Figures $2.3,2.4)$.

To demonstrate the restricted replication of VACV in cells expressing CCR5Y339F, we performed a one-step growth curve, specifically transferring lysates form cells inoculated with VACV to BSC-1 cells for plaque evaluation. The data in Figure 5 indicate that VACV replication is restricted in cells expressing CCR5Y339F but not CCR5, consistent with the infection data (Figure 2.4). 


\section{Figure 2.3}

\section{Introduction of CCR5 but not CCR5.Y339 into primary human T-cells renders}

them permissive for vaccinia virus infection

cDNA for intact CCR5, CCR5Y339F, or vector alone was introduced by electroporation into primary human T-cells and (a) cell surface CCR5 or mutant CCR5 expression determined by flow cytometry: grey solid line represents cells surface CCR5/CCR5Y339F expression while filled (negative) cytograms correspond to IgG reagents alone. (b) Cells were infected with VACV at an m.o.i. of 10, then after 16h the cells were lysed, centrifuged at $1500 \mathrm{rpm}$ for 5 mins and the supernatants transferred onto BGMK cell monolayers. 16h later, the monolayers were fixed and X-gal staining was performed. (c) Cells were infected with VACV at the m.o.i indicated. After 16h cells were lysed and LacZ activity was measured using the B-galactosidase colorimetric assay: Primary T cells; $\quad$ T cells expressing intact CCR5; $\quad$ Ш $\quad$ T cells expressing CCR5Y339F; $\square$ T cells transfected with pEF.BOS vector alone. The data are representative of 3 independent experiments. 
Figure 3 b

a
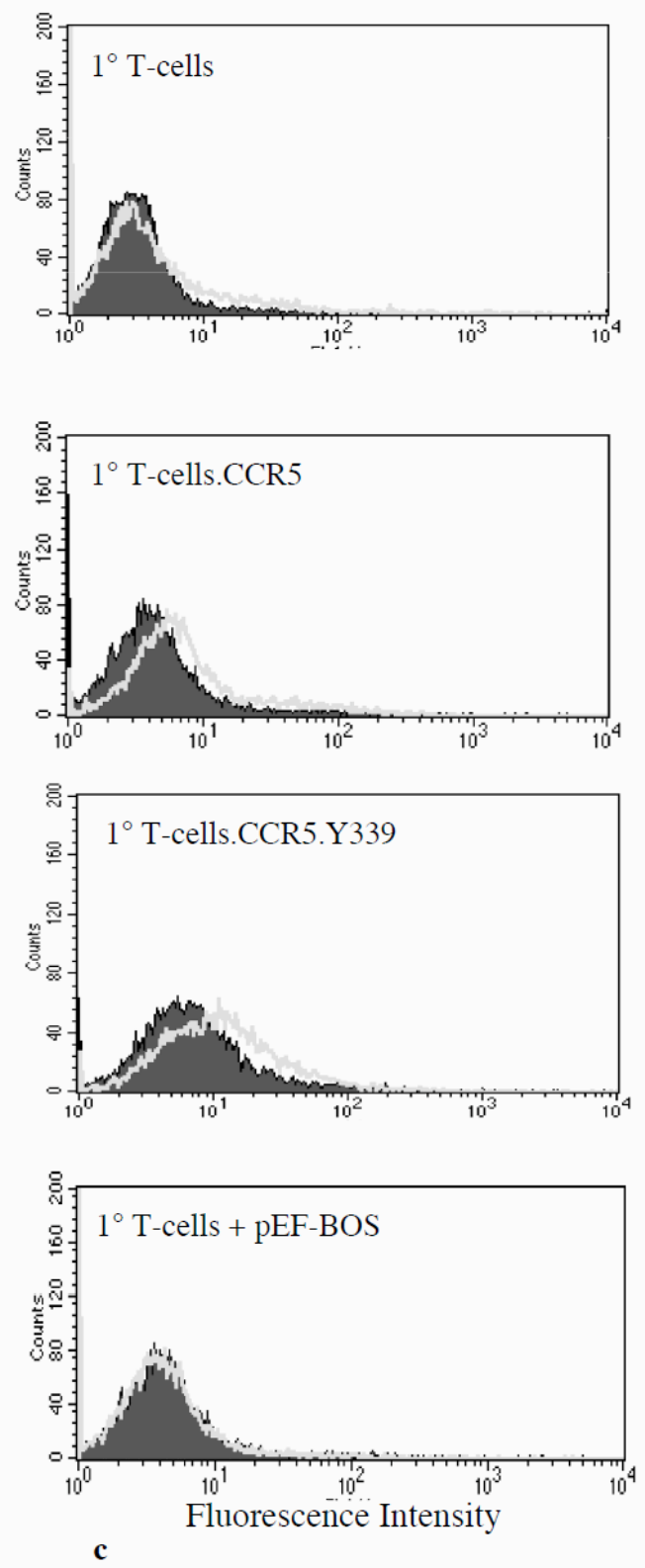
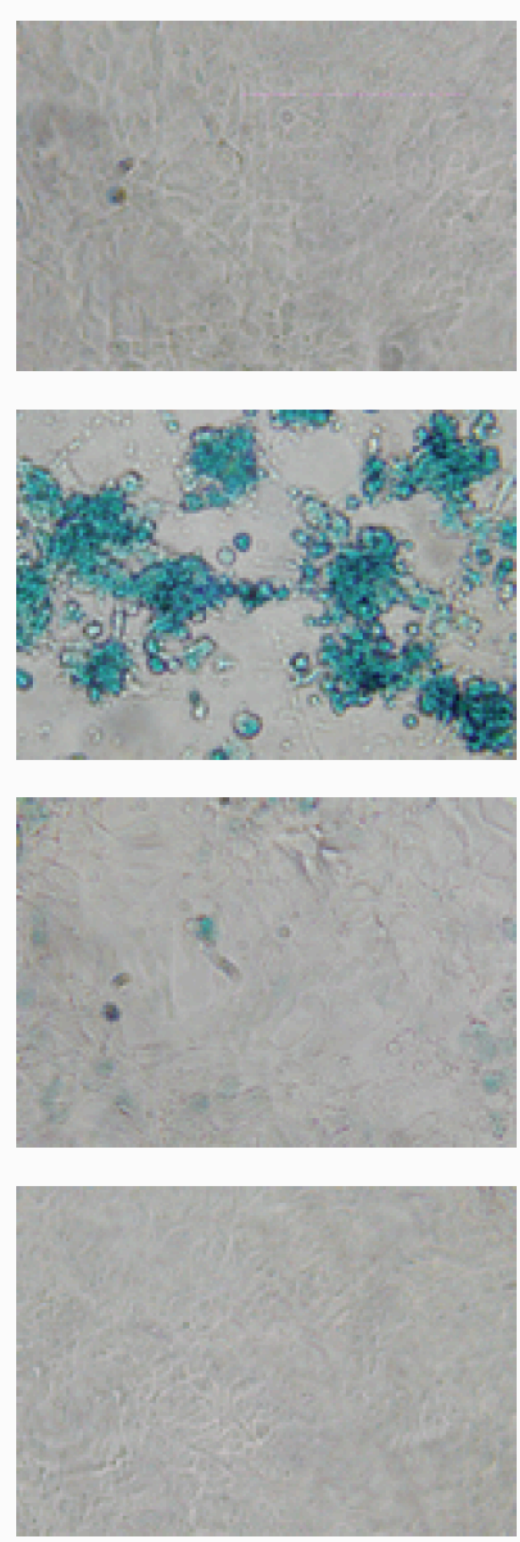

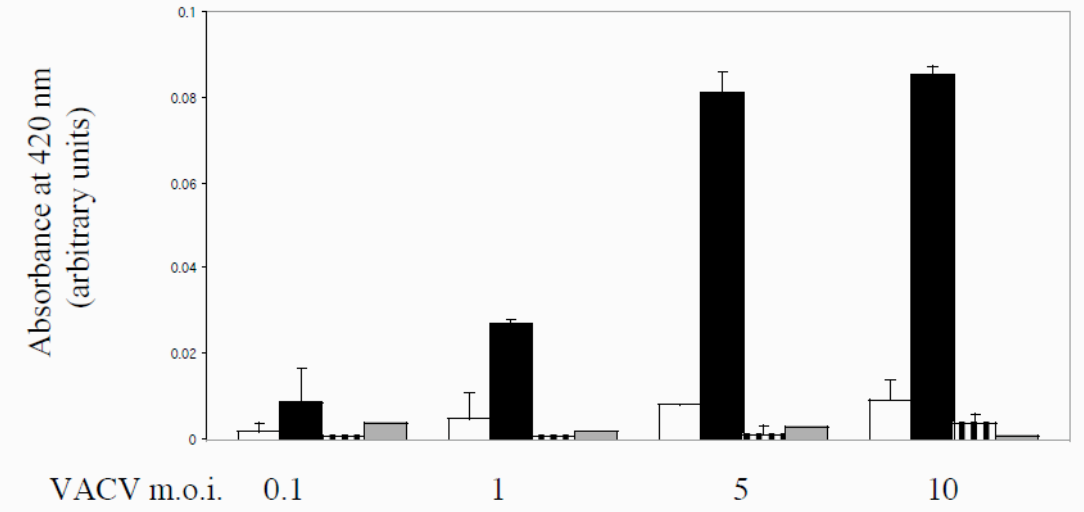




\section{Figure 2.4}

\section{Introduction of CCR5 but not CCR5.Y339 or CCR5.Y $\triangle 3 F$ into PM1 T-cell line renders them permissive for vaccinia virus infection}

cDNA for intact CCR5, CCR5Y339F, CCR5.YA3F or vector alone was introduced by retroviral transduction into PM1 human T-cell line and (a) cell surface CCR5 or mutant CCR5 expression determined by flow cytometry: grey solid line represents cells surface CCR5/CCR5Y339F/CCR5.Y 3 F expression, while filled (negative) cytograms correspond to IgG reagents alone. (b) Cells were infected with VACV at an m.o.i. of 10, then after $16 \mathrm{~h}$ the cells were lysed and LacZ activity was measured using the $\beta$ galactosidase colorimetric assay: The mean $+/-$ S.E. values of quadruplicate assays are shown, representative of 2 independent experiments. 
Figure 4

a
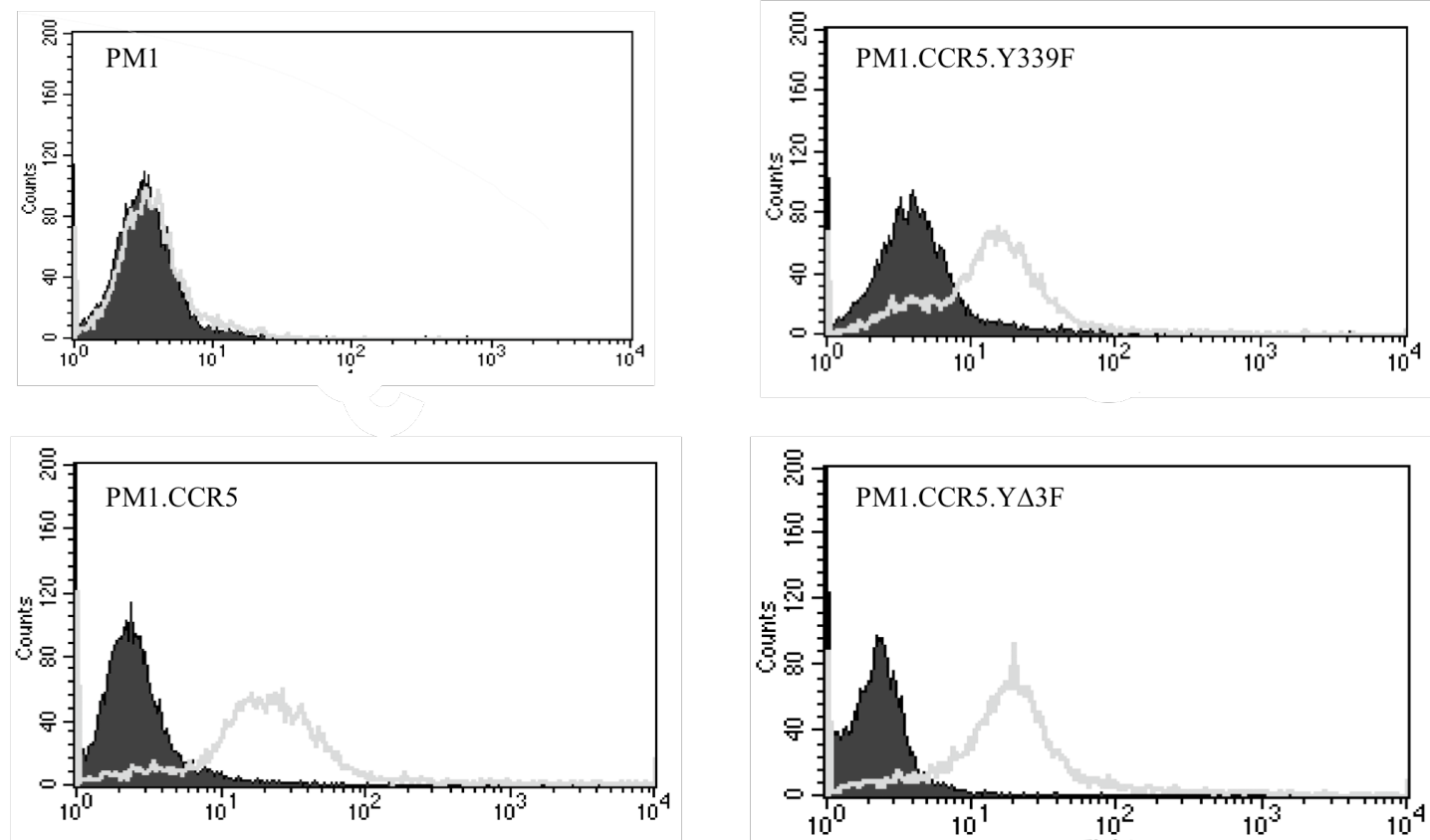

Fluorescence Intensity

b

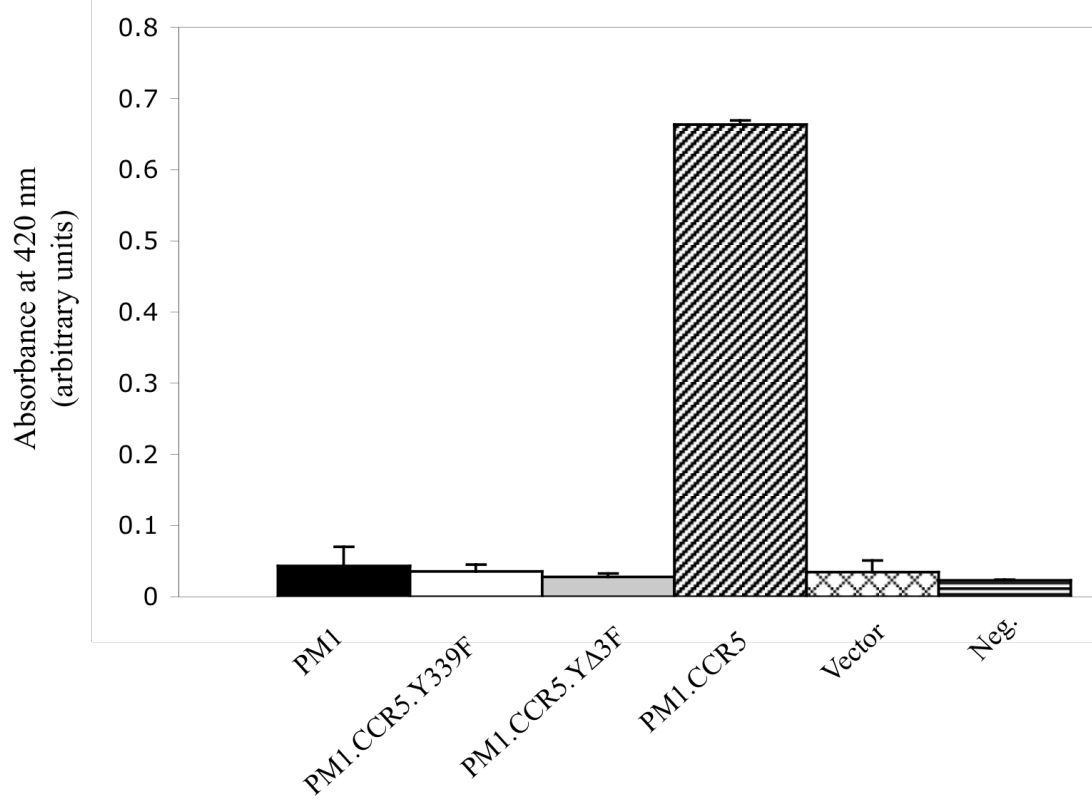




\section{Figure 2.5}

\section{Replication of VACV is restricted in PM1 cells expressing CCR5.Y339F but not CCR5}

PM1.CCR5 or PM1.CCR5.Y339F cells were infected at m.o.i. of 10 with VACV. At the indicated times, cells were harvested and lysed by three successive freeze-thaw cycles. Viral titers were determined by plaque assay on BS-C-1 cells: $\square$ PM1 .CCR5.Y339F; PM1.CCR5. Values are the mean +/- S.E. The data are PM1.CCR5. Values are the mean $+/$ - S.E. The data are representative of 2 independent experiments. ${ }^{*} \mathrm{p}<0.05$ 
Figure 5

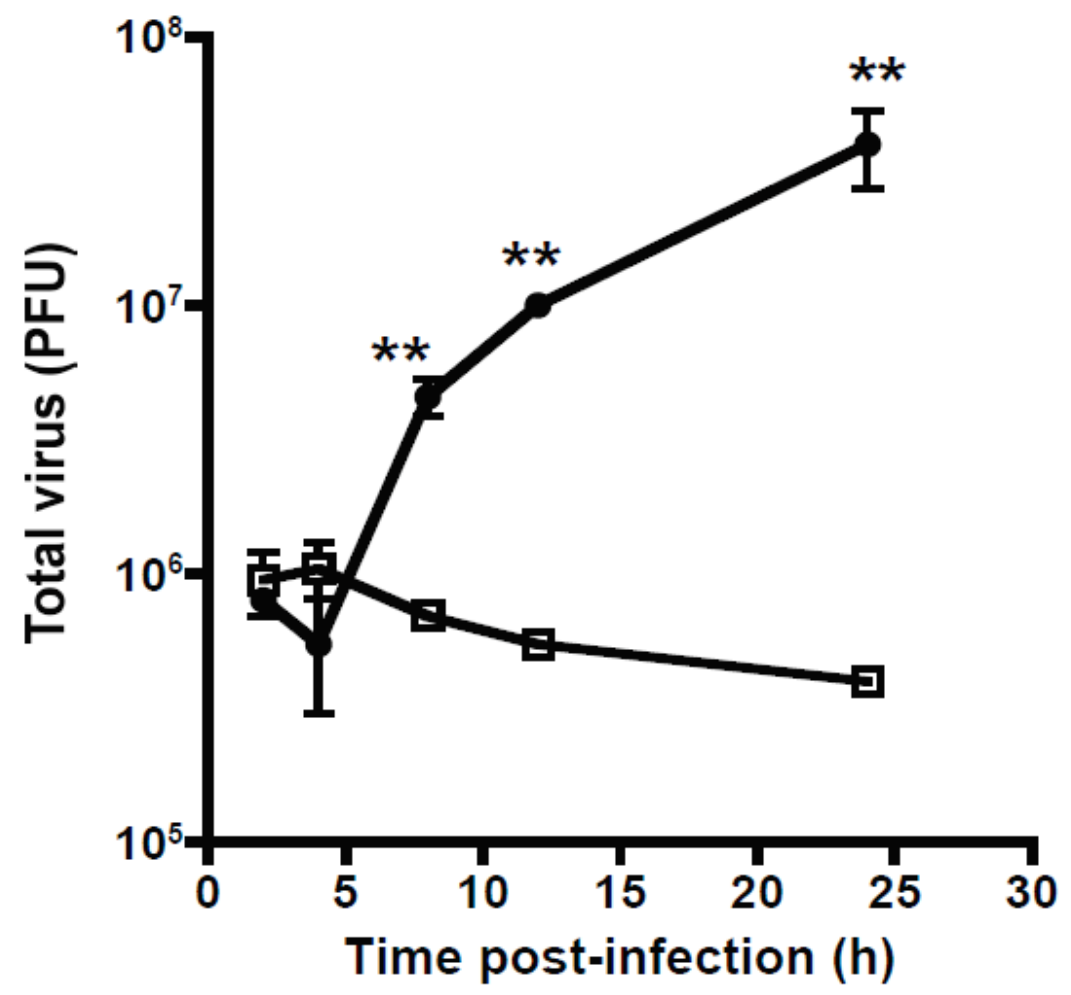




\subsubsection{Absence of VACV late gene transcription in non-permissive cells}

To examine at which stage viral replication is affected in cells expressing CCR5Y339F, we investigated the kinetics of VACV early, intermediate, and late transcription. Cells expressing the intact or the CCR5Y339F mutant were inoculated with 10 m.o.i. of VACV and at 2, 4, 8, 12 and 24 hours cells were harvested for RNA extraction. Primers specific for VACV genes 005R (VACV growth factor), 078R (VACV late transcription factor VLTF-1), and 047R (VACV 11-kDa DNA-binding protein) were designed, representing well-characterized VACV early, intermediate, and late genes, respectively $(28,48)$. PCR data revealed that virus early gene expression was unaffected in cells expressing the mutant receptor, with the $005 \mathrm{R}$ transcript detectable at $2 \mathrm{hr}$ postinoculation.

Notably, expression of the intermediate gene transcript, 078R, was apparent at 2 hr post-inoculation in cells expressing intact CCR5, yet delayed to 4 to 8 in PM1 cells expressing CCR5Y339F. Moreover, whereas expression of the late gene transcript, 047R was initiated between 2 to $4 \mathrm{hr}$ post-inoculation in PM1.CCR5 cells, we observed no evidence of this transcript in the cells expressing the mutant CCR5Y339F (Fig. 2.6).

\subsubsection{A CCR5 antagonist inhibits vaccinia virus infection}

In subsequent studies we employed the CCR5 antagonist TAK 779 (38). This small chemical molecule binds a cavity between transmembrane helices 1, 2, 3 and 7 of CCR5, near the extracellular surface (172) and may induce a conformational change in CCR5 that perturbs the ligand binding sites and inhibits ligand induced signaling. 


\section{Figure 2.6}

Absence of VACV late gene transcription fails in PM 1.CCR5Y339F cells.

PM1.CCR5.Y339F and PM1.CCR5 cells were infected with 10 m.o.i. of VACV. At 0, 2, 4, 8, 12 and $24 \mathrm{hr}$ post-inoculation cells were harvested for RNA extraction. RTPCR for VACV genes 005R (a), 078R (b), and047R (c), representing vaccinia virus early, intermediate, and late genes, respectively was performed. A probe specific for human $\beta$-actin was included to monitor transcription of a cellular mRNA (d). 
Figure 6

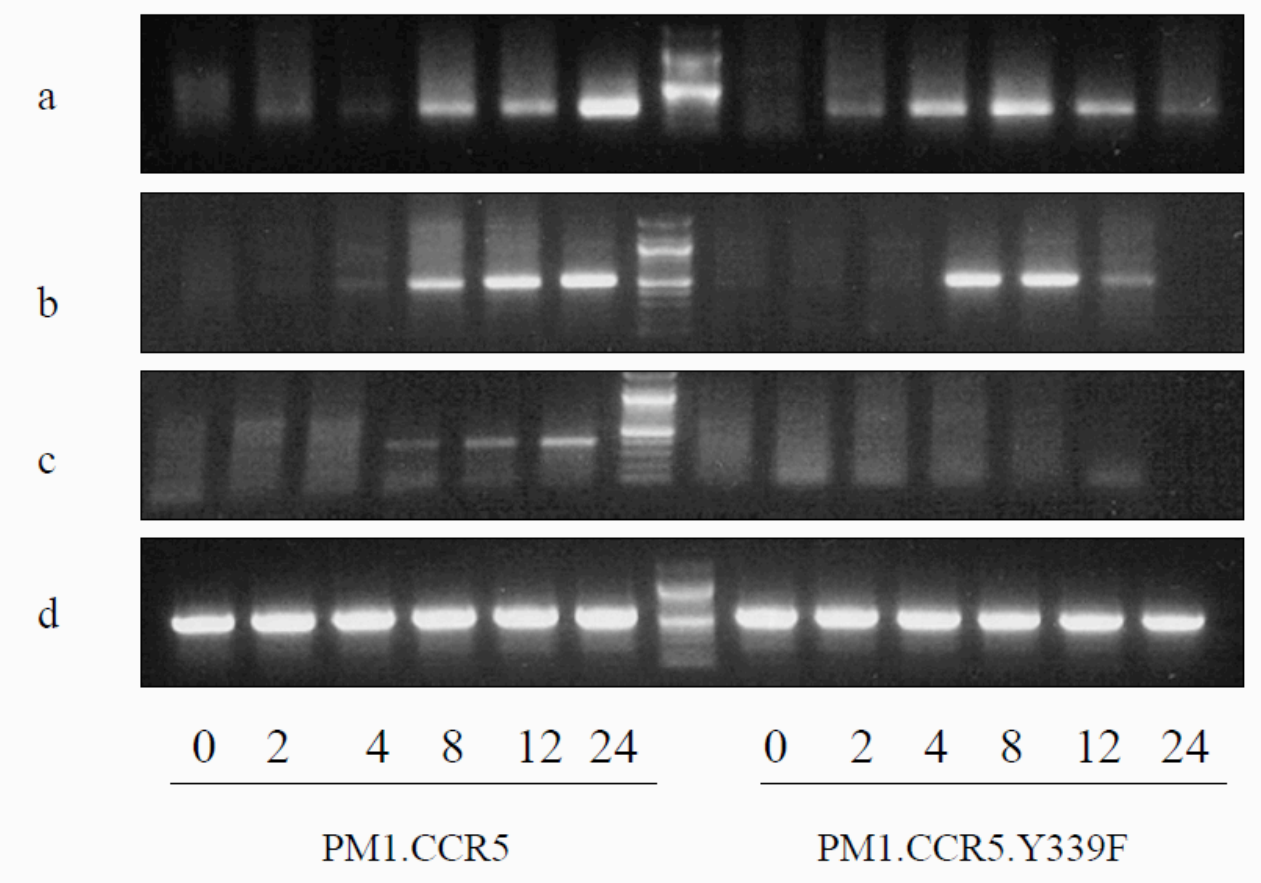


We observed a marked reduction in viral infection in PM1.CCR5 cells pretreated with TAK 779 (Figure 2.7a). The dose required to maximally inhibit vaccinia infection, 1nM, is in keeping with the HIV reported $\mathrm{EC}_{50}$ of $1.2 \mathrm{nM}$ and $\mathrm{EC}_{90}$ of $5.7 \mathrm{nM}(38)$. These data further suggest that CCR5 activation is necessary to permit VACV infection and that TAK 779 may interfere with critical CCR5-mediated signaling events that are required for viral infection.

\subsubsection{Vaccinia virus induces tyrosine phosphorylation of cellular intermediates}

Chemokine activation of CCR5 results in the engagement of G-protein-coupled events as well as G-protein-independent, tyrosine phosphorylation signaling effectors (reviewed in (759)). Accordingly, we examined viral infection under conditions where either G-protein or phosphotyrosine signaling pathways are inhibited. Specifically, in dose-response experiments, PM1.CCR5 cells were pretreated with the tyrosine kinase inhibitor herbimycin A, or with pertussis toxin. Cells were then infected with VACV and $16 \mathrm{hr}$ post infection, cell lysates were assayed for viral infection. In contrast to treatment with pertussis toxin that had no effect, herbimycin A inhibited VACV infection in a dosedependent manner (Figure 2.7b, c). These data are in agreement with published reports that the activation of tyrosine kinase and not G-protein coupled signal transduction contributes to both myxoma and VACV infection(314, 353, 385, 416).

When whole cell lysates from vaccinia-infected PM1.CCR5 cells were resolved by SDS PAGE and immunoblotted with antibodies against phosphotyrosine, a general increase in vaccinia-dependent induction of tyrosine phosphorylation in several protein 
bands was consistently observed. Notably, cell lysates from infected PM1.CCR5Y339F cells exhibited no increase in tyrosine phosphorylation. Chemokine activation of CCR5 triggers the rapid phosphorylation of receptor associated Jaks (757) and CCR5-CCL5 interactions have been known to recruit Jak 1 (561), Jak 2 and Jak3 (757) in a cellspecific manner. Examination of the phosphorylation status of different Jak proteins revealed a vaccinia-dependent induction of Jak2 tyrosine phosphorylation within 1 minute of viral adsorption in PM1.CCR5 cells (Figure 2.7d). To examine the role of Jak 2 activation in mediating vaccinia infection, PM1.CCR5 cells were pretreated with the Jak 2 inhibitor AG490 (tyrophostin $\left.\mathrm{B} 42, \mathrm{IC}_{50} \sim 10 \mu \mathrm{M}(435,482)\right)$ prior to vaccinia adsorption. AG490 inhibited VACV-inducible phosphorylation of Jak2 (Figure 2.7d). In parallel, a dose-dependent inhibition of VACV infection was observed (Figure 2.7e). These results are consistent with earlier findings of myxoma virus activation of the JakStat pathway (416). 


\section{Figure 2.7}

Pharmacological inhibition of vaccinia virus infection in PM1.CCR5 cells

PM1.CCR5 cells were either left untreated or treated for $1 \mathrm{hr}$ with varying doses of

TAK799 (a), pertussis toxin (Ptx) (b), herbimycin A (HA) (c), or 16 hrs with AG490 (e), prior to VACV adsorption . Cells were infected at an m.o.i. of 10, LacZ activity was measured $16 \mathrm{hr}$ post infection using the B-galactosidase colorimetric assay. (d) PM1.CCR5 cells were either left untreated (U), infected with VACV at an m.o.i of 10 for 1 minute, or treated with AG490 at $100 \mu \mathrm{M}$ concentration for $16 \mathrm{hr}$ prior to infection with vaccinia at an m.o.i of 10 for 1 minute. Cell lysates were immunoprecipitated (IP) with anti-Jak2 antibodies. Lysates were resolved on SDS-PAGE and immunoblotted (WB) with antiphosphotyrosine (4G10) antibodies. The blot was stripped and reprobed for Jak2. The data are representative of 2 independent experiments. 
Figure 7

a
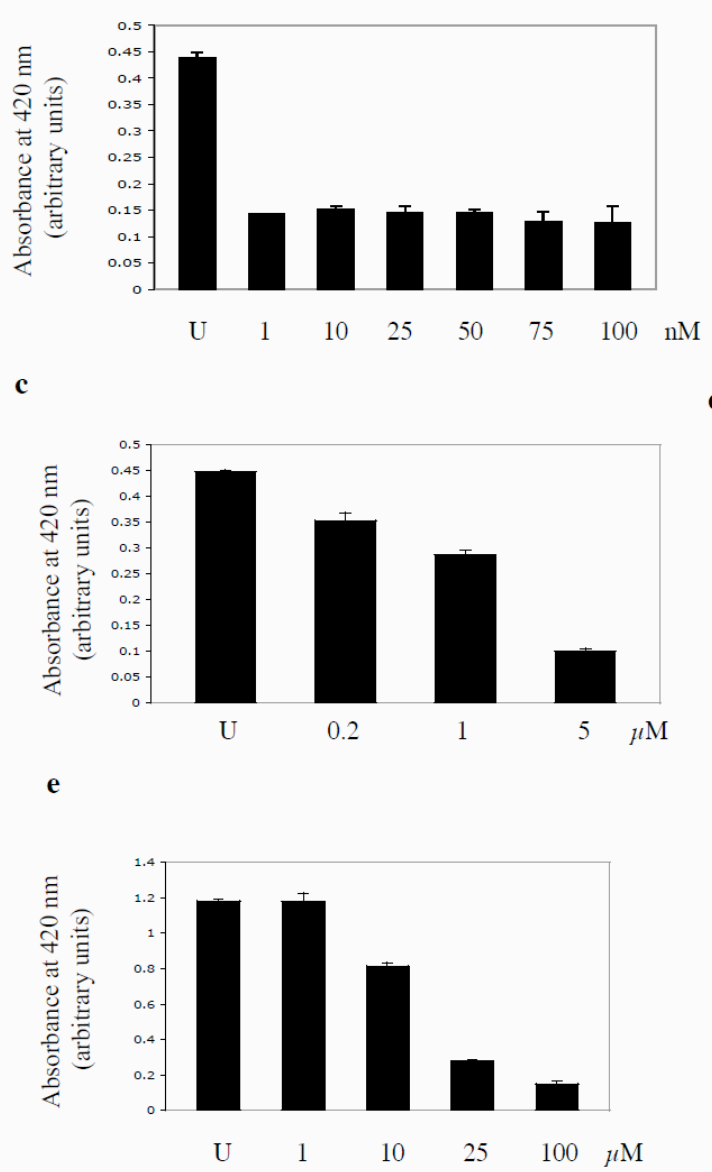

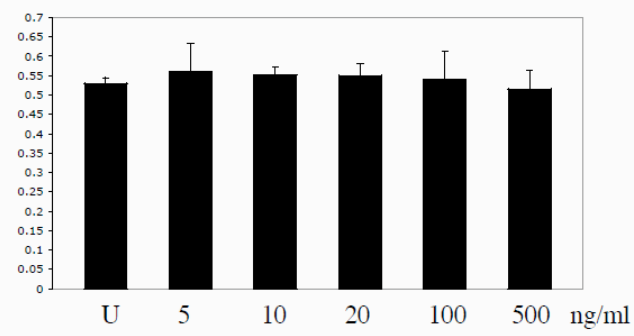

d

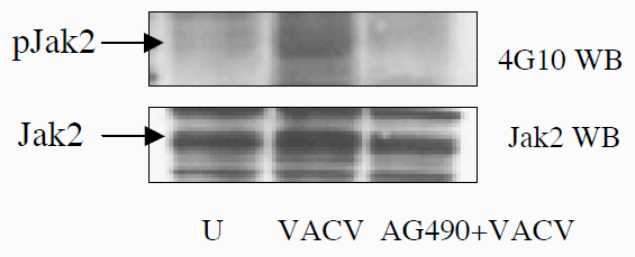




\subsubsection{Vaccinia virus induces tyrosinephosphorylation of signaling effectors in permissive cells}

We examined the effects of vaccinia infection on IRS-2 tyrosine phosphorylation, a downstream effector of Jaks. IRS proteins participate in signal transduction by providing tyrosine-phosphorylated motifs for direct binding to the $\mathrm{SH} 2$ domains of various signaling proteins. The data in Figure 8a show that vaccinia infection of PM1.CCR5 cells results in the rapid phosphorylation of IRS-2 at 1 minute that is sustained for at least 120mins post-infection. Expression of the CCR5Y339F mutant in these cells results in no discernible vaccinia induced phosphorylation of IRS-2 above baseline levels (Figure 2.8a). Since IRS proteins associate with PI3'K, SHP2, Fyn, Nck, and Grb2 (744), we infer that vaccinia activation of IRS-2 has the potential for engagement of a diverse range of signaling cascades. Indeed, we provide evidence for vaccinia-dependent induction of tyrosine phosphorylation of the adaptor protein Grb2 in the permissive PM1.CCR5, by 1 minute post-infection (Figure 2.8b). In replicate experiments we observe that this VACV-inducible phosphorylation of Grb2 is transient, diminishing by 5 minutes post-infection, then increasing once again between 12 and 15 minutes post-infection. Notably, we consistently observe basal levels of phosphorylation of Grb2 in the PM1.CCR5.Y339F cells that are not increased further on VACV infection (Figure 2.8b). Grb2 activation may have very specific implications for vaccinia infection, especially since its phosphorylation has been shown to reduce SH3-dependent binding to Sos (369) and is thus thought to represent a potential regulatory point for signal transduction that may regulate the specificity of SH3 domain interactions (66). The viral protein A36R, responsible for 
actin polymerization during viral egress has been shown to interact with Grb2 (597), followed by a co-localization with N-WASP, and the actin-related protein complexes 2 and 3 (Arp2/3) (205). Since protein tyrosine phosphorylation was shown to be required for the formation of membrane protrusions mediating virus entry into HeLa cells (385), the tyrosine phosphorylation of Grb2 observed in PM1.CCR5 cells may be linked to actin reorganization upon viral entry.

In addition to its SH2 domain that can associate with IRS-2, the Grb2 adaptor molecule contains multiple SH3 domains, capable of binding downstream signaling proteins involved in the regulation of cell metabolism, growth and differentiation. Grb2 is known to participate in the Ras cascade, due to its association with the guanine nucleotide exchange factor Sos, and thus may link signaling effectors to the MAP kinase pathway. Indeed, we were able to demonstrate a vaccinia-induced increase in extracellular signal regulated kinase (Erk) tyrosine phosphorylation in the permissive PM1.CCR5 cells. Figure 8c shows that in the permissive PM1.CCR5, by 1 minute post-infection VACV-inducible phosphorylation of Erk1/2 is present and reaches a maximum at 15 minutes post-infection. In comparison, we consistently observe basal levels of phosphorylation of Erk1/2 in the PM1.CCR5.Y339F cells that are not increased further on VACV infection (Figure 2.8c). This activation of the MAPK pathway appears to be virus-specific since U0126, a MAPK/Erk1,2 inhibitor, has been shown to attenuate vaccinia infection, but increase myxoma virus infection in permissive 3T3 cell lines (314). Furthermore the role for activation of intracellular signaling events in permissive cells was demonstrated by a myxoma-dependent activation of the Ser/Thr kinase PAK-1 (314). Activation of this intermediate in non- 
permissive NIH3T3.CD4.neo cells was greatly reduced and the expression of the autoinhibitory domain of PAK-1 in permissive cells was shown to inhibit myxoma infection, suggesting a regulatory role for this kinase in determining the extent of poxviral infection. As for IRS-2 and Grb2, there is no discernible VACV-inducible phosphorylation of both Erk1 and Erk2 in the non-permissive PM1.CCR5.Y339F cells (Figure 2.8c). 


\section{Figure 2.8}

\section{Vaccinia virus induces tyrosine phosphorylation of IRS-2, Grb2 and Erk 1/2 in permissive but not in non-permissive cells}

PM1.CCR5 or PM1.CCR5Y339F cells were left untreated (Ø) or infected with VACV at an m.o.i. of 10 for the times indicated. Cell lysates were either (a) resolved by SDSPAGE and immunoblotted (WB) with 4G10 anti-phosphotyrosine antibody, then stripped and reprobed for IRS-2, or (b) immunoprecipitated with an anti-Grb2 antibody, the solubilized immunoprecipitate resolved by SDS-PAGE and immunoblotted with 4G10 antibody; then stripped and reprobed for Grb2, or (c) resolved by SDS-PAGE and immunoblotted with anti-phospho-Erk 1/2 antibody, then stripped and reprobed for Erk1/2. The data are representative of 2 independent experiments. 
Figure 8

a

$\begin{array}{llllllllllllllllll}\mathrm{VACV} & \varnothing & 1 & 5 & 10 & 15 & 30 & 60 & 120 & \varnothing & 1 & 5 & 10 & 15 & 30 & 60 & 120 & \mathrm{~min}\end{array}$
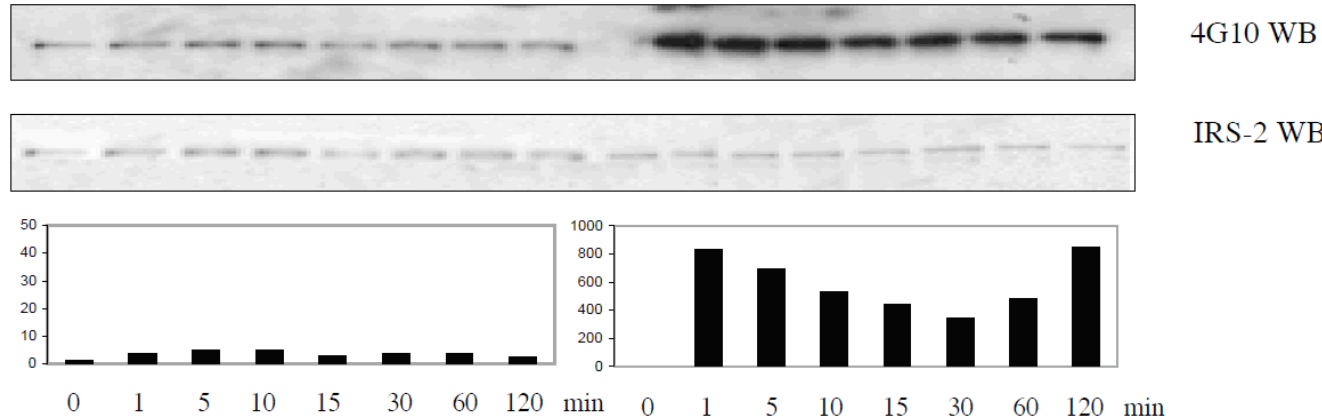

b

$\begin{array}{llllllllllllllllll}\mathrm{VACV} & \varnothing & 1 & 5 & 10 & 15 & 30 & 60 & 120 & \varnothing & 1 & 5 & 10 & 15 & 30 & 60 & 120 & \mathrm{~min}\end{array}$
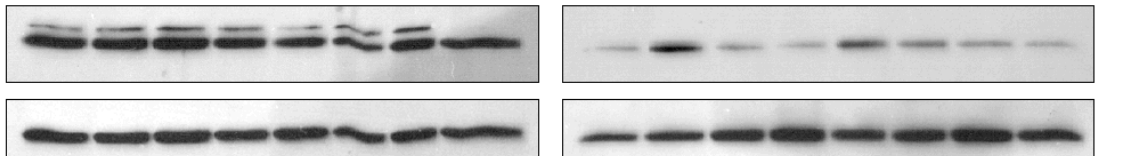

Grb2 IP 4G10 WB
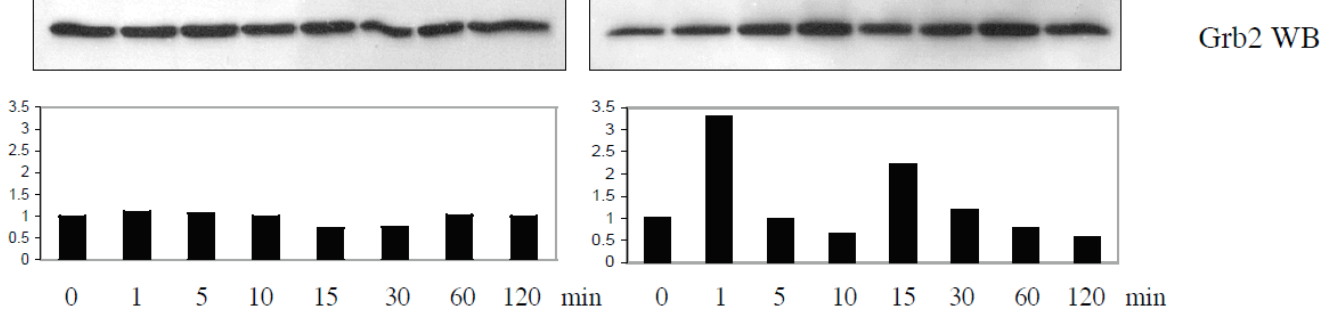

c

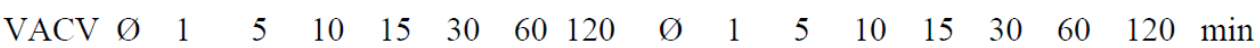
$+-\ldots-\ldots$ pErk1/2 WB

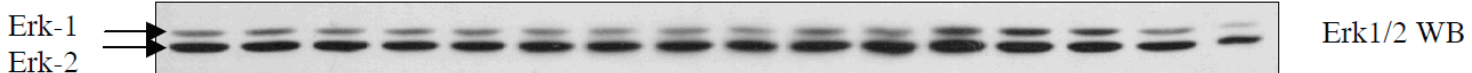
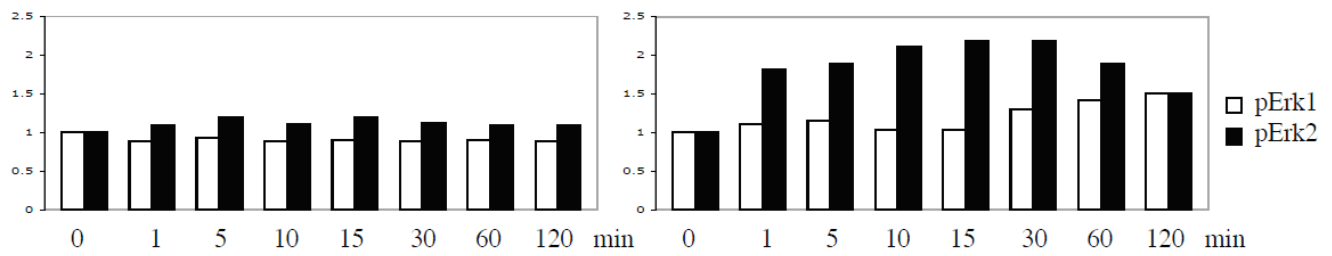

PM1.CCR5.Y339F

PM1.CCR5 


\subsubsection{Vaccinia virus co-localizes with $C C R 5$ in permissive cells}

Using A5L-EGFP IMV, we examined VACV entry into both permissive and nonpermissive cells, whilst at the same time visualizing the movement/location of CCR5, using confocal microscopy. Cells were infected on ice at an m.o.i of 10 with IMV infectious particles, then warmed at 37 degrees for the times indicated in the series of panels in Figure 2.9. As shown, VACV enters the non-permissive PM1 cells within 5 minutes of warming to 37 degrees and is distributed in the cell for up to 60 minutes (Figure 2.9b). Since CCR5 is not expressed in these cells, staining with anti-mouse Cy3 antibody directed against mouse anti-human CCR5 antibody, revealed no CCR5. By contrast, PM1.CCR5 cells express CCR5 at the cell surface, visualized using the red Cy3 antibody (Figure 2.9c.d). When PM1.CCR5 cells are infected with VACV, the virus enters the cells within 5 minutes of warming to 37 degrees. Notably, at 5 minutes, CCR5 remains externally expressed. By 15 minutes, both the virus and CCR5 co-localize inside the cell. By 60 minutes, the majority of CCR5 relocates to the cell surface. In Figure 9e,f data are presented that illustrate that CCR5.Y339F remains externally expressed in the non-permissive PM1.CCR5Y339F cells, for up to 60 minutes post-infection/warming to 37 degrees. As for the non-permissive PM1 cells, VACV enters the PM1.CCR5Y339F cells on warming to 37 degrees, rapidly, and remains distributed throughout the cell for up to 60 minutes. The data indicate that VACV enters both permissive and non-

permissive cells, yet the sequence of signaling events described in Figure 2.8 and the permissive phenotype are associated with intracellular co-localization of VACV and CCR5.

Since there is accumulating evidence to suggest that IMV and EEV virions enter cells 
differently and with differing kinetics, using GFP-tagged virions (F13L-GFP) we examined EEV entry into both permissive and non-permissive cells. Cells were infected on ice at an m.o.i of 10 with EEV VACV infectious particles. Cells were harvested at 2, 4,8,12 and $24 \mathrm{hr}$ post-infection and infectious virus associated with cells was measured by plaque assay. A one-step growth curve revealed that, similar to results in Figure 2.5, EEV virions enter both permissive and non-permissive PM1 cells and replication is restricted in cells expressing CCR5Y339F but not CCR5

(Supplementary Figure 2.1). Moreover, using confocal microscopy, we have evidence of GFP-tagged virions cells expressing CCR5 but not CCR5Y339F (Supplementary Figure 2.2).

In a final series of experiments, we compared the ability of ectopically expressed CCR5 and CCR5Y339F to internalize following infection of cells with IMV infectious particles. In agreement with the confocal studies described in Figure 2.9, flow cytometric analysis of cell surface expressed CCR5 revealed that VACV infection leads to the rapid internalization of CCR5 within minutes of exposure to virus, which is maximal at 12-15 minutes post-infection, then CCR5 returns to the cell surface by 30 minutes (Figure 2.10a). By contrast, cells expressing CCR5Y339F do not internalize the mutant receptor in response to VACV infection (Figure 2.10b). 


\section{Figure 2.9}

CCR5 and IMV vaccinia virus co-localize in permissive PM1.CCR5 T cells

PM1 (a,b), PM1.CCR5 (c,d) or PM1.CCR5Y339F (e,f) cells were infected with A5L-

EGFP IMV vaccinia virus for $1 \mathrm{hr}$ at $4^{0}$, the cells warmed to $37^{0}$, and at the time points indicated fixed and stained for CCR5 (Cy3 antibody), then analyzed by confocal microscopy, as described in Materials \& Methods. The cell boundary is outlined in panel (a,b and $\mathbf{d})$ as a dashed line. The data are representative of 2 independent experiments. Optical sections collected at $0.5 \mu \mathrm{m}$ intervals through the entire cell. 
Figure 9

a

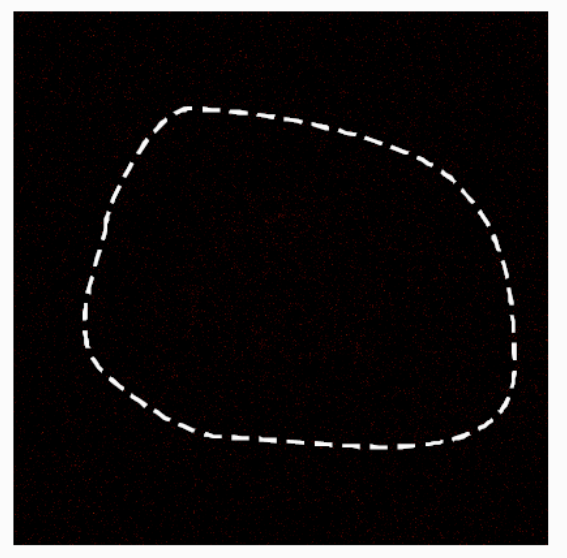

CCR5

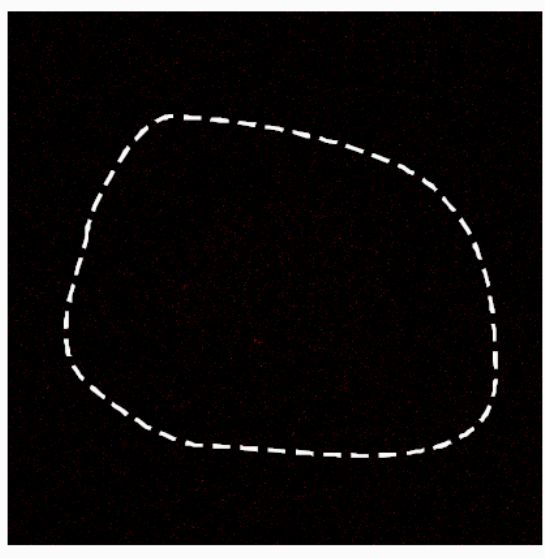

A5L-EGFP

IgG Ctrl.

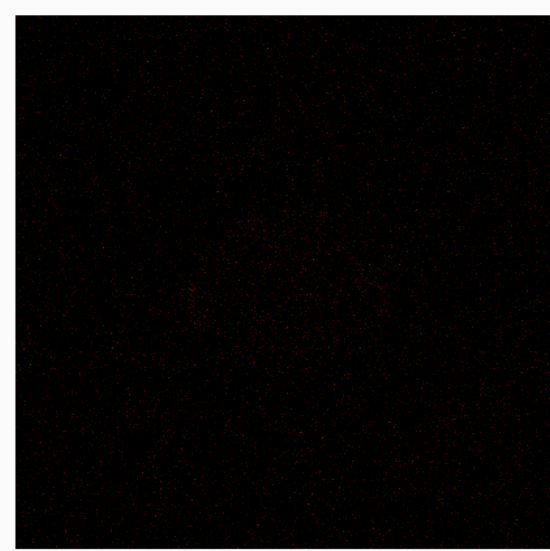


b
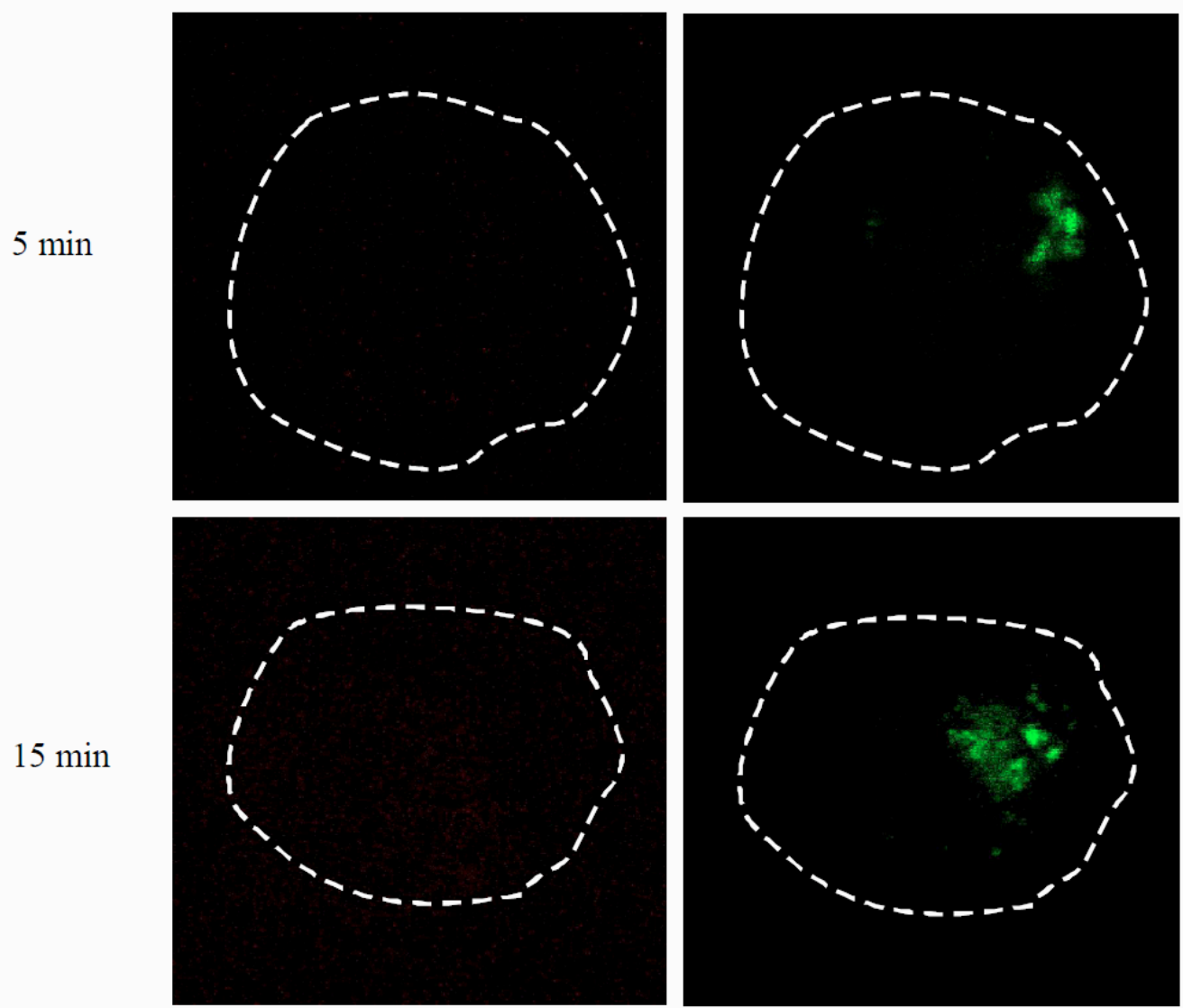

$15 \mathrm{~min}$

$60 \mathrm{~min}$
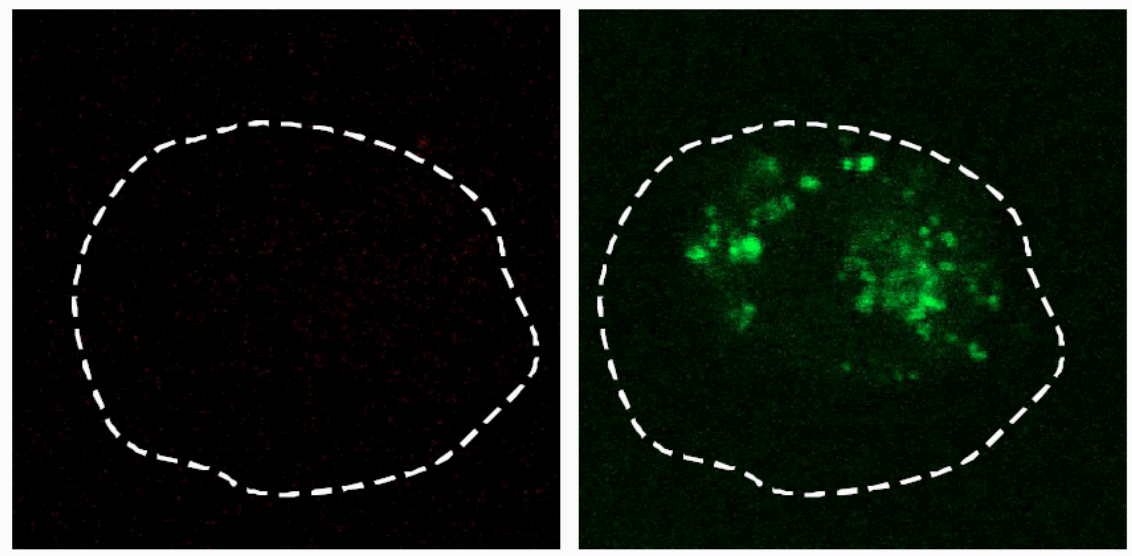

CCR5

A5L-EGFP 
c

$0 \mathrm{~min}$

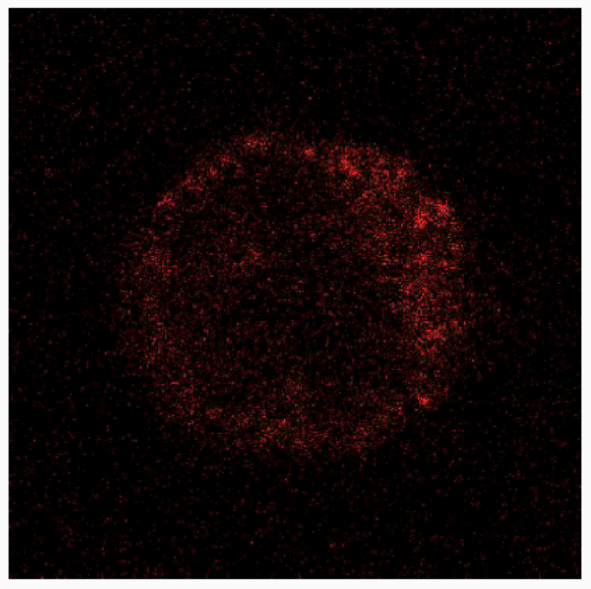

CCR5

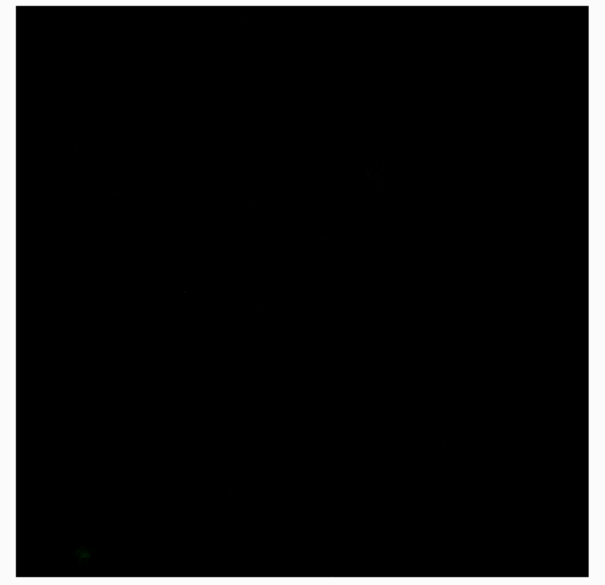

A5L-EGFP

IgG Ctrl.

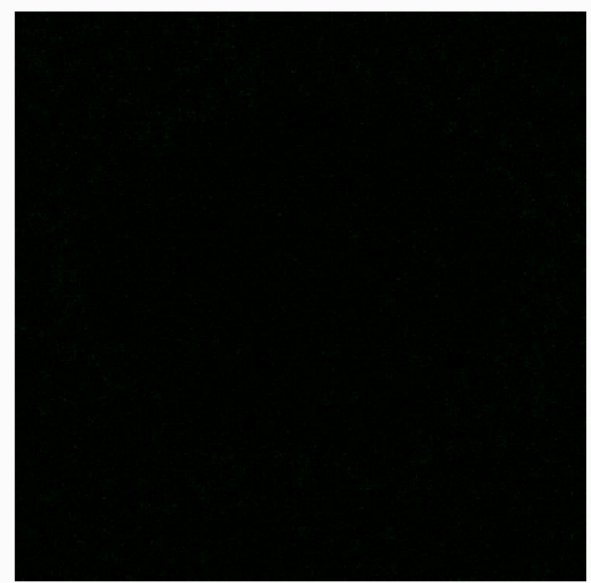


d

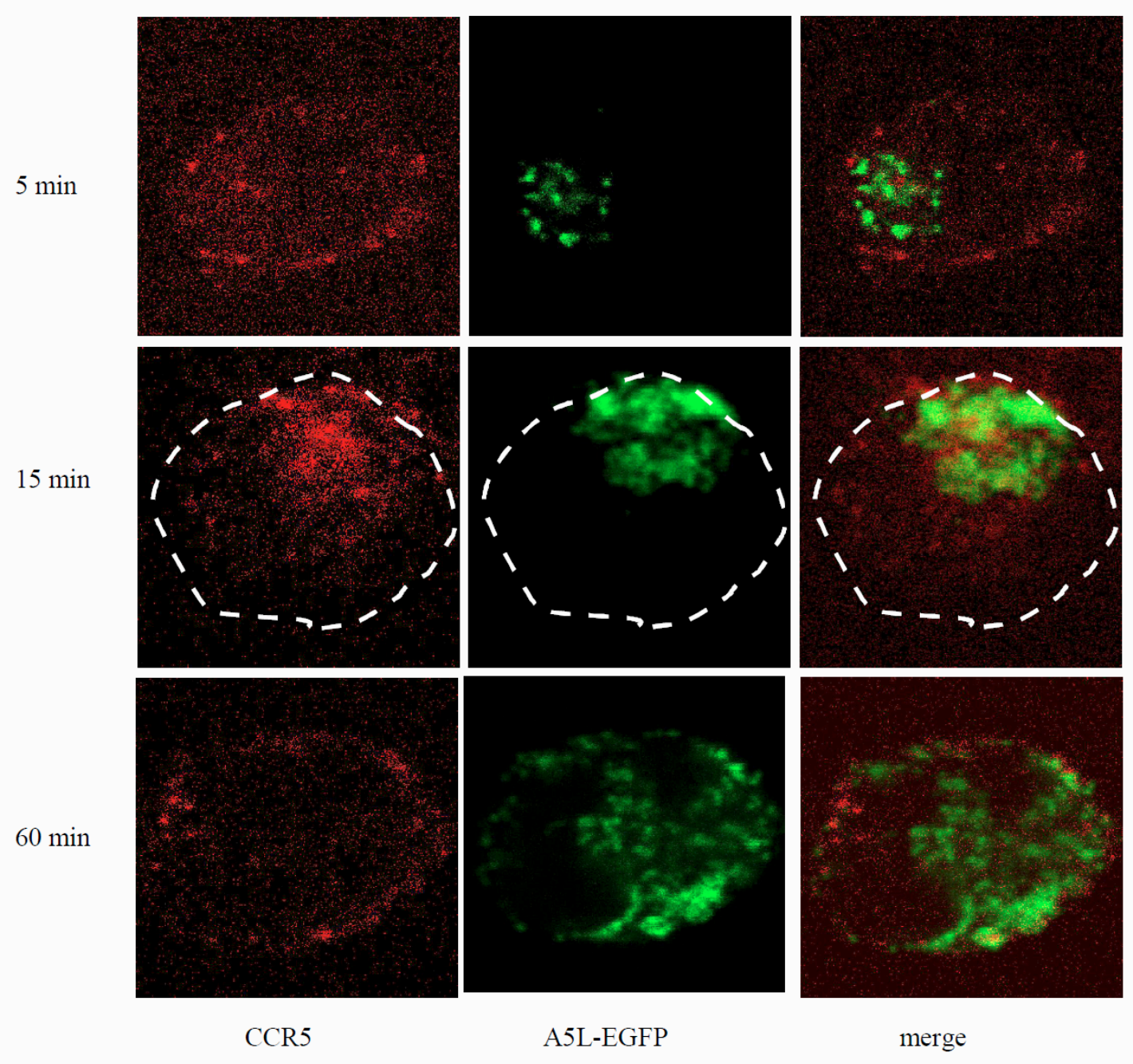




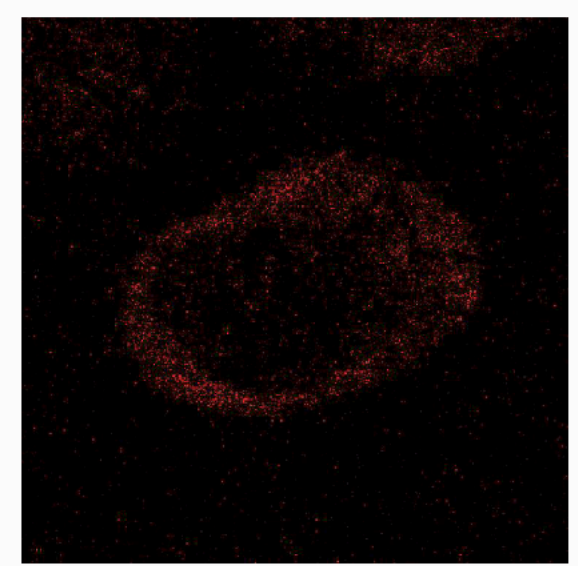

CCR5

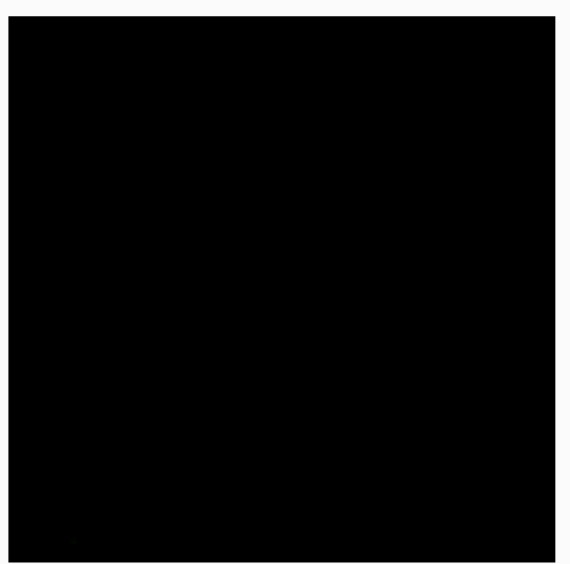

A5L-EGFP

IgG Ctrl. 


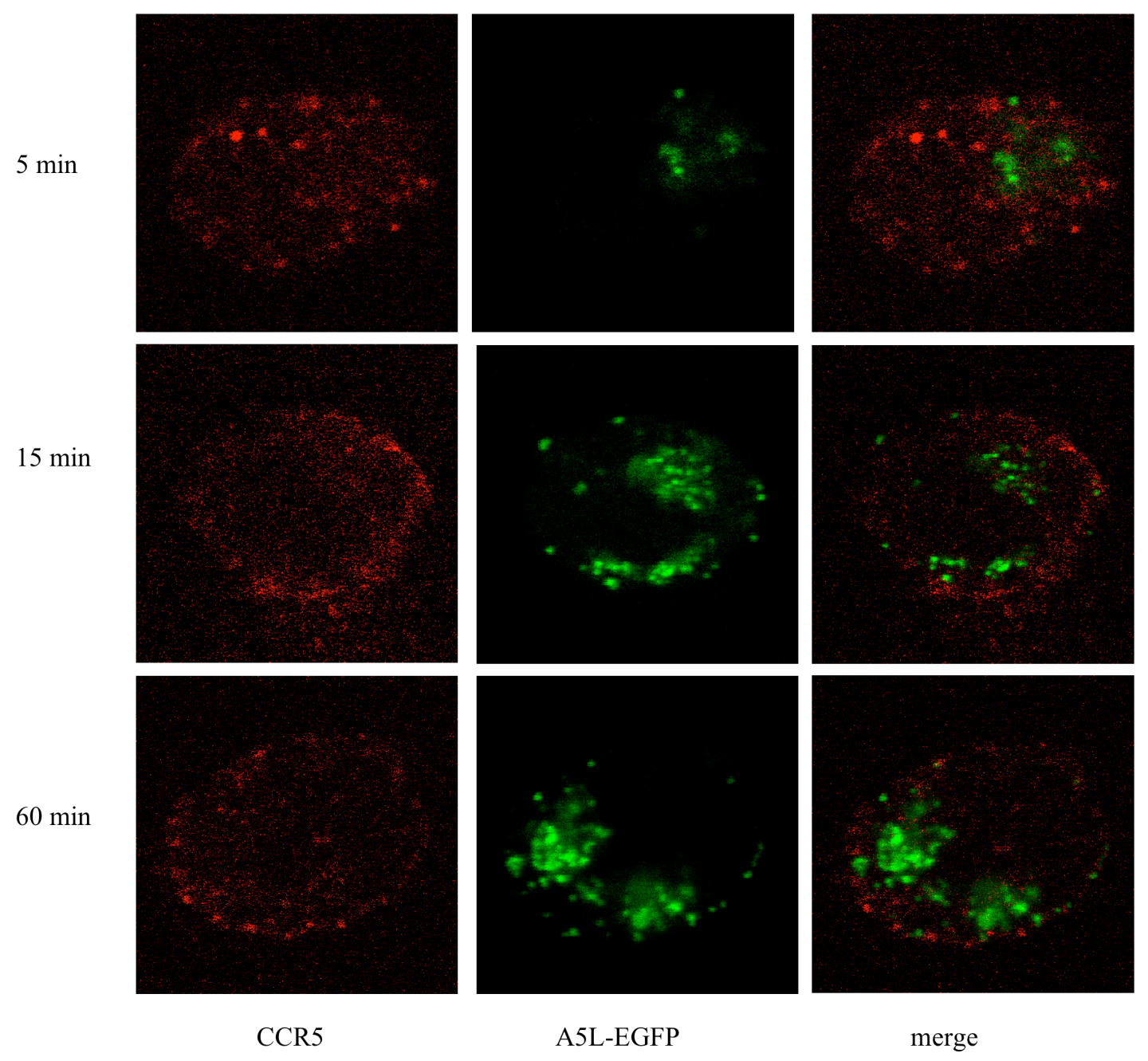


Figure 2.10

CCR5 internalizes in response to vaccinia virus in PM1.CCR5 but not PM1.CCR5Y339F cells

PM1.CCR5 cells were infected with VACV at an m.o.i. of 10 (a), for the times indicated, then ectopically expressed CCR5 quantitated by flow cytometry using anti-human CCR5 antibody and FITC anti-mouse IgG. Similarly, PM1.CCR5Y339F cells were infected with VACV (b) for the times indicated and cell surface CCR5Y339F quantitated by flow cytometry. The percentage of cell surface CCR5 or CCR5Y339F was determined relative to the total ectopic expression in untreated cells. The data are representative of 2 independent experiments. 
Figure 10

a

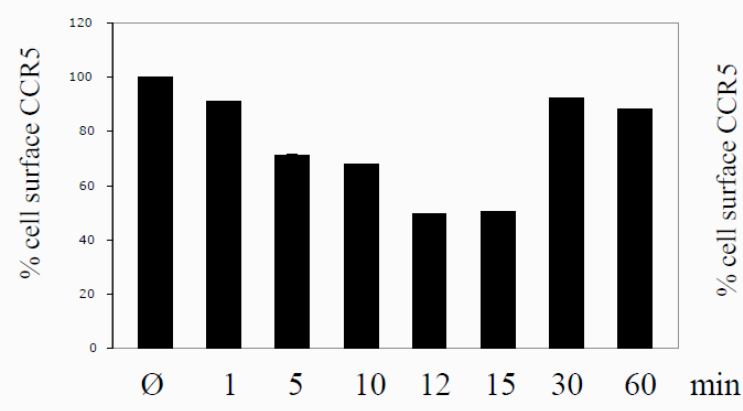

b

PM1.CCR5.Y339F+ 10 m.o.i VACV

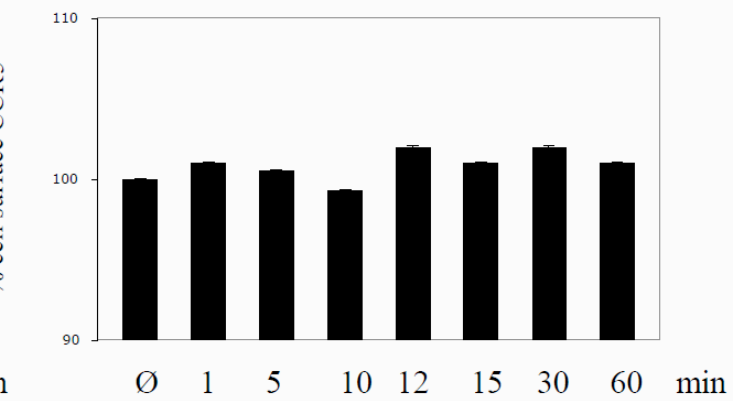




\subsection{DISCUSSION}

Viewed altogether, these results allow us to infer that not only is tyrosine phosphorylation of CCR5 important for VACV infection in the context of permissive mouse fibroblast and T cells expressing CCR5, but in particular Y-339 in the C-terminal tail of the receptor is crucial. Interestingly, in other studies we have evidence that Y-339 in CCR5 is also critical for CCL5-inducible activation of various signaling effectors, including Erk1/2 (unpublished). Since NIH 3T3.CD4.CCR5 cells are rendered less permissive by knockdown of CCR5, and NIH3T3.CD4.neo cells are rendered permissive by ectopic expression of CCR5, but not a tyrosine phosphorylation mutant CCR5 (CCR5Y339F), this argues against the notion of permissiveness being entirely dependent on the lineage derivation of the NIH3T3.CD4.CCR5 cells, independent of ectopic expression of the specific transfected receptors, as has been suggested (314). Phosphorylation of the CCR5 Y-339 receptor residue may be an early event in a virusinduced signaling cascade that accompanies infection and Y-339 may be responsible for recruitment of signaling intermediates that we have shown to be induced by vaccinia early post infection. Subsequent activation of crucial downstream kinases such as PAK1 and other serine/threonine kinases may, in turn, promote some early aspect of viral infection. The implications are that viral activation of CCR5 activates signaling events that involve tyrosine phosphorylation, thereby rendering a cell permissive for viral infection. Our data infer that in these 3T3 mouse fibroblasts, expression of CCR5 contributes to the permissive phenotype. Moreover, we provide evidence that expression of CCR5 in primary human $\mathrm{T}$ cells or an immortalized human $\mathrm{T}$ cell line, renders these $\mathrm{T}$ cells permissive for VACV replication, and that Y-339 is critical. Interestingly, a 
recently published report suggests that VACV requires a specific receptor to infect primary human leukocytes. Activated $\mathrm{T}$ cells, but not resting $\mathrm{T}$ cells, were found to be permissive for VACV infection (114). Notably, susceptibility to infection is determined by expression of a receptor that is induced de novo upon $\mathrm{T}$ cell activation. When primary human $\mathrm{T}$ cells derived from peripheral blood mononuclear cells (PBMC) are activated by exposure to phytohaemagglutinin and IL-12 (305), we observe the inducible de novo ecptopic expression of CCR5 (data not shown). Viewed altogether with the data presented herein, the implications are that CCR5 renders primary human $\mathrm{T}$ cells permissive for VACV infection. Since VACV will replicate in cells and cell lines that lack CCR5, it is intriguing to speculate that virus activation of different receptors may occur to initiate host signaling events.

Of note, the myxoma virus infection defect in NIH3T3.CD4.neo cells occurs at an intracellular stage following virion binding, entry and early gene expression, but prior to the later events of DNA replication and late gene expression (314). Our data provide evidence that VACV enters both permissive and nonpermissive T-cell lines and that replication is restricted in nonpermissive cells, specifically at the stage of late viral gene expression. We show that VACV early gene expression is evident for up to $24 \mathrm{~h}$ postinfection in both permissive and nonpermissive cells. Early VACV gene expression is unique in that it does not require the de novo synthesis of DNA or proteins, since the template, viral RNA polymerase, and early transcription factors are present within the infectious virus particle (88). New progeny IMVs are generated yet are not released from infected cells until lysis, except as EEV (638). Thus, new rounds of early VACV gene expression may persist in infected cells, prior to progeny release, deriving from the new 
progeny. With nonpermissive cells, we observed that infectious virus may be harvested for up to $24 \mathrm{~h}$ postinfection (Figure 5), implying that not all IMV cores enter the viral replication cycle and/or that entry may be asynchronous or delayed. Thus, prolonged early VACV gene expression in nonpermissive cells may reflect this asynchronous commencement of early viral replication. The products of early gene transcription are required for intermediate VACV gene expression $(568,594,718,719)$. Accordingly, protracted intermediate VACV gene expression reflects the corresponding broad time frame for early gene expression.

In the context of CCR5, permissiveness for virus replication correlates with internalization of the receptor and the rapid virus-inducible tyrosine phosphorylation of a number of different signaling effectors. By use of time course studies, we have provided evidence for the intracellular peripheral colocalization of VACV and CCR5 in permissive cells. Since VACV enters cells regardless of whether or not CCR5 is ectopically expressed, CCR5 is not associated with viral entry. In nonpermissive cells, the apparent block in viral replication we observed coincides with a block in late gene expression. It is intriguing to speculate that IMV colocalization with CCR5 at the plasma membrane results in activation of CCR5 at Y339 either through direct contact between IMV core proteins and CCR5 or by mediation due to IMV interacting with other cellular factors that are recruited to the intracellular region of CCR5. CCR5 activation leads to a cascade of tyrosine phosphorylation signaling events that, apparently, prescribe cellular events that are necessary for late VACV gene expression. Whether conserved amino acid motifs in the intracellular domains of different receptors are associated with viral activation or whether a broad spectrum of tyrosine kinases that can associate with different receptors 
and invoke their activation are rendered kinase active by viral factors remains to be determined and is the subject of our ongoing investigations.

\subsection{MATERIALS AND METHODS}

\subsubsection{Cells and virus}

Murine fibroblast NIH3T3.CD4.CCR5 and NIH3T3.CD4.neo cells generated from NIH3T3 parental cells and are distinguished by the unique presence of a neomycin resistance cassette in the NIH3T3.CD4.neo variant and were obtained from D. Littman (New York University). Phoenix cells were a gift from Dr. Josef Penninger (Institute of Molecular Biotechnology, Austrian Academy of Sciences, Vienna, Austria). All cells were maintained in Dulbeco's Modified Eagle Medium (DMEM) (Gibco-BRL), supplemented with $10 \%$ fetal calf serum (FCS) (Gibco-BRL), 100 units/ml penicillin (Gibco-BRL) and $100 \mathrm{mg} / \mathrm{ml}$ streptomycin (Gibco-BRL), and grown at $37^{\circ} \mathrm{C}$ in an atmosphere of $5 \% \mathrm{CO}_{2}$.

$\mathrm{T}$ cells were purified from peripheral blood (healthy adult donors) by FicollPaque (Amersham Biosciences) density gradient separation, the leukocytes collected at the interphase were incubated with an anti-CD32 blocking antibody, then T cells purified by negative selection using the StemCell Technologies magnetic bead separation system and a cocktail of antibodies directed against CD19, CD56, CD66b, as per the manufacturer's instructions. Purified T cells were cultured in DMEM $+10 \% \mathrm{FCS}+100$ units $/ \mathrm{ml}$ penicillin, $100 \mathrm{mg} / \mathrm{ml}$ streptomycin overnight. 
Human PM1 T cells were obtained from the National Institutes of Health AIDS Research and Reference Reagent Program, and were maintained in RPMI 1640 (GibcoBRL) with $10 \%$ FCS, 100 units $/ \mathrm{ml}$ penicillin, $100 \mathrm{mg} / \mathrm{ml}$ streptomycin and $25 \mu \mathrm{g} / \mathrm{ml}$ Plasmocin (InvivoGen).

Vaccinia Virus-VACV65 (modified Western reserve strain) containing an E.coli B-galactosidase cassette driven by a late viral promoter was a gift from Dr. Grant McFadden (Robarts Research Institute, University of Western Ontario). Purified IMV harvested from infected green monkey kidney cells (BGMK) that were subjected to 2 freeze-thaw cycles, sonication and dounce homogenization. Supernatants containing crude viral preparations were collected by centrifugation of the cell homogenates at $10,000 \mathrm{rpm}$ for $1 \mathrm{hr}$ at $4^{\circ} \mathrm{C}$. Since the EEV form accounts for approximately $1 \%$ of progeny, no attempt was made to fractionate EEV and IMV. (Mock infections were conducted using BGMK culture supernatants that were subjected to the same process, but the BGMK cells were not infected with VACV). Viral titers were determined by duplicate plaque assays. Specifically, 1:10 serial dilutions of stock virus were adsorbed onto BGMK cells for $1 \mathrm{hr}$, the cultures incubated for $48 \mathrm{hrs}$, then stained for X-gal to reveal plaques.

A5L-EGFP VACV was a gift from Dr. Geoffrey Smith (Department of Virology, Faculty of Medicine, Imperial College of London)(112). A5L-EGFP VACV was grown in HeLa cells, purified by sedimentation through a $36 \%$ sucrose cushion(363) and the number of virus particles determined from the optical density at $260 \mathrm{~nm}\left(1 \mathrm{U}=1.2 \times 10^{10}\right.$ particles)(506). Using this value, the particle: P.F.U. ratio of A5L-EGFP VACV was estimated to be $50: 1$. 


\subsubsection{Measurements of viral infectivity}

The extent of vaccinia infection in different cell types was determined by detecting the vaccinia-associated $\beta$-galactosidase activity by means of $\mathrm{X}$-gal staining as described(370). Images were captured with a Leica DMIL inverted microscope (Leica, Willowdale, Canada) equipped with a Zeiss digital AxioCam camera using Axio Vision 2.05 software. Acquired images were processed with Adobe Photoshop version 7 (Adobe Systems Inc., San Jose, CA).

In all our studies, $ß$-galactosidase activity was directly proportional to the extent of viral replication, as determined from replicate plaque assays undertaken to titer the Bgal cassette-containing vaccinia strain using the X-gal colorimetric staining assay.

Additionally, to examine the effects of the pharmacological inhibitors herbimycin A (Calbiochem), pertussis toxin (Calbiochem), TAK 779 (Serono Pharmaceuticals), and AG490 (Calbiochem) on the extent of viral infection, cells were pretreated with various doses of theses inhibitors, then infected with VACV as described above. The effects of these inhibitors on viral replication were measured using the $\beta$-galactosidase colorimetric assay.

\subsubsection{Analysis of CCR5 surface expression by flow cytometry}

Cell surface expression of human CCR5 was determined by flow cytometry using anti-human CCR5 (BD Pharmingen) and FITC anti-mouse IgG (eBioscience). Staining was conducted according to the manufacturer's protocol. Cells were gated based on forward and side scatter. Flow cytometric data were obtained using FACSCalibur (BD), 
and analyzed using the CELLQuest software. Notably, the anti-human CCR5 antibody recognizes ectopically expressed intact CCR5, CCR5Y339F, CCR5Y307F and CCR5Y $\triangle 3 \mathrm{~F}$.

\subsubsection{Cell lysis and immunoblotting}

To prepare whole cell lysates (WCLs), $10^{7}$ cells were infected with the equivalent of $10^{8}$ infectious units (m.o.i of 10) of VACV for the indicated times. Cells were washed in cold PBS and lysed as described previously (416). Immunoprecipitations and immunoblotting using enhanced chemiluminescence were performed as described (416), using the following antibodies : 4G10 (UBI), anti-IRS-2 (Upstate Biotech.), anti-Erk 1/2 (Santa Cruz), anti-phospho-Erk1/2 (Santa Cruz), anti-Grb2 (Santa Cruz), anti-Jak2 (Santa Cruz).

\subsection{5. siRNA transfection}

siRNA duplexes were synthesized and purified by Qiagen Inc. The CCR 5 target sequences were: siRNA1 (5' - CUCUUGACAGGGCUCUAUU-3') and siRNA2 (5'AAGCCAGGACGGUCACCUU-3'). 2 x 10 ${ }^{6}$ NIH3T3.CD4.CCR5 cells were transfected with a mixture of $10 \mu \mathrm{g}$ siRNA1 and $5 \mu \mathrm{g}$ siRNA2 or a control, scrambled siRNA by electroporation (BTX T820 electroporator). The settings were $475 \mathrm{~V}, 1 \mathrm{msec}$ and 4 pulses. At 24 and $48 \mathrm{hrs}$ after transfection cell surface CCR5 expression was determined by flow cytometry using the anti-human CCR5 antibody (BD Pharmingen). 


\subsubsection{CCR5 mutagenesis}

The pEF-BOS-CCR5 carrying the human CCR5 gene was obtained from Dr. Martin Opperman (University of Gottingen, Germany). Human CCR5 was subcloned into pUMFG retroviral vector (a gift from Dr. Jeffery Medin, Division of Experimental Therapeutics, Toronto General Research Institute). CCR5 mutagenesis was performed on the pEF-BOS-CCR5 and pUMFG-CCR5 templates. Initially, single Y127F, Y307F, and Y339F mutations were introduced using the QuickChange Site-Directed Mutagenesis Kit (Stratagene) and the appropriate mutagenic primers:

\section{Y127F}

(forward) CTC CTG ACA ATC GAT AGG TTC CTG GCT GTC GTC C

(reverse) GGA CGA CAG CCA GGA ACC TAT CGA TTG TCA GGA G

\section{Y307F}

(forward)CGG GGA GAA GTT CAG AAA CTT CCT CTT AGT CTT CTT CC

(reverse) GGA AGA AGA CTA AGA GGA AGT TTC TGA ACT TCT CCC CG

\section{Y339F}

(forward) GCG AGC AAG CTC AGT TTT CAC CCG ATC CAC TGG GG

(reverse) CCC CAG TGG ATC GGG TGA AAA CTG AGC TTG CTC GC

The triple tyrosine CCR5 mutant, CCR5 Y $\triangle 3 \mathrm{~F}$, was generated in three subsequent rounds of mutagenesis, using the relevant primer sets and the following cycling parameters: denaturation at $95^{\circ} \mathrm{C}$ for $30 \mathrm{sec}$, followed by 16 cycles of denaturation at $95^{\circ} \mathrm{C}$ for 30 
sec, annealing at $55^{\circ} \mathrm{C}$ for $1 \mathrm{~min}$, and elongation at $68^{\circ} \mathrm{C}$ for $14 \mathrm{~min}$, and a final extension at $68^{\circ} \mathrm{C}$ for 7 minutes. After each round of mutagenesis, the location of the appropriate nucleotide changes and the lack of additional mutations were confirmed by sequencing (ACGT Corporation, Toronto).

\subsubsection{Introduction of CCR5, CCR5.Y307F, CCR5.Y339F and CCR5.Y $\triangle 3 F$ into cells}

NIH3T3.CD4.neo cells were transfected with $5 \mu \mathrm{g}$ of either intact or mutant CCR5 cDNA using the Fugene 6 transfection reagent, according to the manufacturer's protocol (Roche). T cells purified from human peripheral blood were transfected with $30 \mu \mathrm{g}$ of either intact or mutant CCR5 cDNA by electroporation (BTX T820 electroporator 600V, $99 \mu \mathrm{sec}$ and 10 pulses). At 3 hrs post-transfection, cell surface CCR5 expression was determined by flow cytometry using the anti-human CCR5 antibody (BD Pharmingen). The amphotropic packaging cell line Phoenix was transfected by the calcium phosphate/chloroquine method. At $48 \mathrm{~h}$ post-transfection, the viral supernatant was collected and used for transfection of PM1 cells as described (Kinsella \& Nolan, 1996). Positive transfectants were FACS sorted using anti human CCR5 antibody.

\subsubsection{Immunohistochemical analysis and confocal microscopy}

PM1, PM1.CCR5 and PM1.CCR5.Y339F were infected with A5L-EGFP VACV and stained for CCR5 by immunohistochemistry as described (753). Specifically, CCR5 was visualized using an anti-mouse Cy3 antibody (Ameresham Biosciences, cardiff, UK) directed against the mouse anti-human CCR5 antibody. Images were collected using an upright Leica SP2 confocal laser-scanning microscope (Leica Microsystems Heidelberg 
GmbH, Mannheim, Germany), 100X oil immersion lens (1.4 NA) and 4X digital zoom. Laser excitations were $488 \mathrm{~nm}(\mathrm{Ar} / \mathrm{Kr})$ and $543 \mathrm{~nm}(\mathrm{He} / \mathrm{Ne})$, attenuated to $10 \%$ and $50 \%$, respectively, by way of an acoustal-optical transmission filter (AOTF). Sequential scan mode was used to eliminate cross talk of detected signals, which were filtered between $500-530 \mathrm{~nm}$ and 560-660 $\mathrm{nm}$. Image resolution was 512x512 (12 bit) and line averaging (4x) was used. Optical sections were collected at $0.5 \mu \mathrm{m}$ intervals through the entire cell.

\subsubsection{Reverse Transcription PCR (RT-PCR)}

$10^{7}$ cells were infected with VACV at a m.o.i. of 10 , then lysed at various times post-infection using the Qiagen QIA-shredder columns and RNA isolated using the Qiagen RNeasy mini kit (as per the manufacturer's instructions). cDNA was synthesized by annealing $2 \mathrm{ug}$ of $\mathrm{RNA}$ at $65^{\circ} \mathrm{C}$ for $5 \mathrm{~min}$ in the presence of oligodT and reverse transcription (RT) was performed at $25^{\circ} \mathrm{C}$ for $10 \mathrm{~min}, 37^{\circ} \mathrm{C}$ for $50 \mathrm{~min}$ and $70{ }^{\circ} \mathrm{C}$ for 15 $\min$.

PCR was performed using following primer sets:

005R primers:

(forward) 5'-TTA TCT GAT GTT GTT GTT GTT CGC-3'

(reverse) 5'- GTT TAG TTC GTC GAG TGA AC-3'

078R primers:

(forward) 5'-CGA TAA ACT GCG CCA AAT TG-3'

(reverse) 5'- CAT AAT AGC CAA ATG CTG ATG-3' 
047R primers:

(forward) 5'-ATT CTC ATT TTG CAT CTG CTC-3'

(reverse) 5'- CTA GAA GCT ACA TTA TCG CG-3' 
2.7. Supplementary Figures 


\section{Supplementary Figure 2.1}

\section{Replication of EEV is blocked in the cells expressing CCR5.Y339F but not CCR5}

PM1.CCR5 or PM1.CCR5.Y339F cells were infected at m.o.i. of 10 with VACV. At the indicated times, cells were harvested and lysed by three successive freeze-thaw cycles. Viral titers were determined by plaque assay on BS-C-1 cells: $\square$ PM1 .CCR5.Y339F; OPM1.CCR5.

Values are the mean $+/-$ S.E. The data are representative of 2 independent experiments. $* * \mathrm{p}<0.05$ 


\section{Supplementary Figure 1}

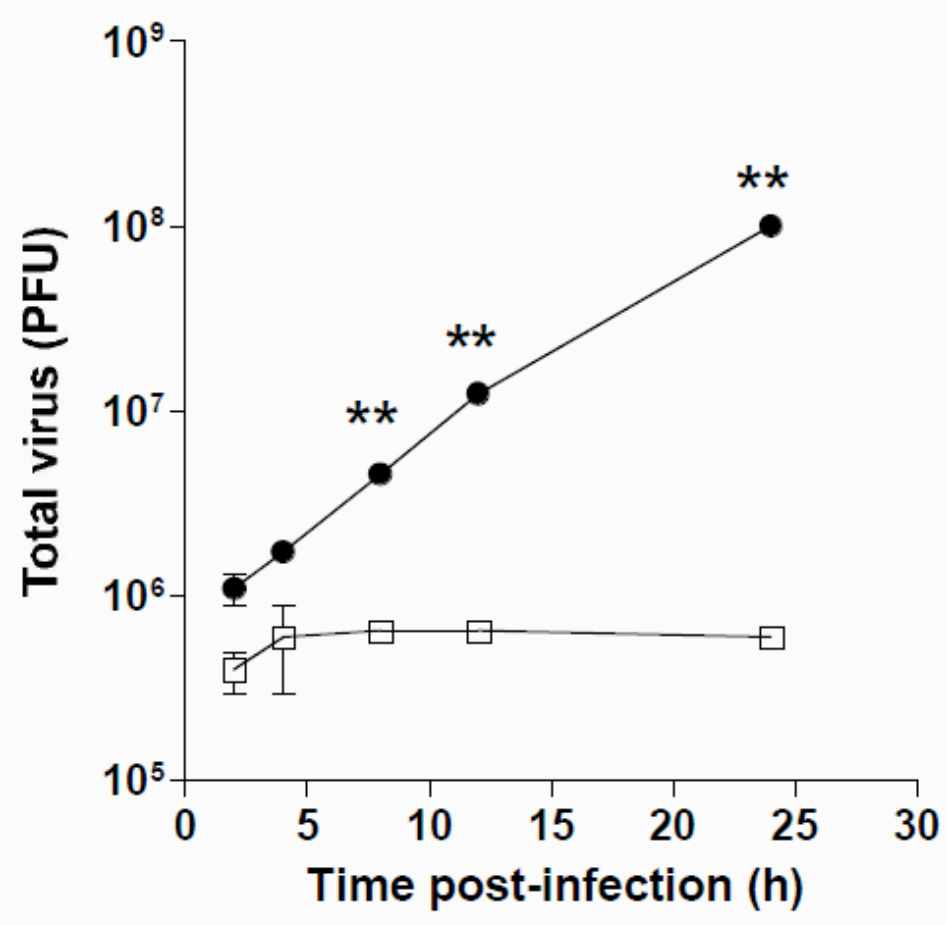




\section{Supplementary Figure 2.2}

EEV replication is restricted in the cells expressing CCR5.Y339F but not CCR5

PM1.CCR5 (A) and PM1.CCR5.Y339F (B) were infected with vF13L-GFP

at m.o.i. Of 10. After $24 \mathrm{~h}$ the cells were analyzed by confocal microscopy.

Green, GFP 
Supplementary Figure 2
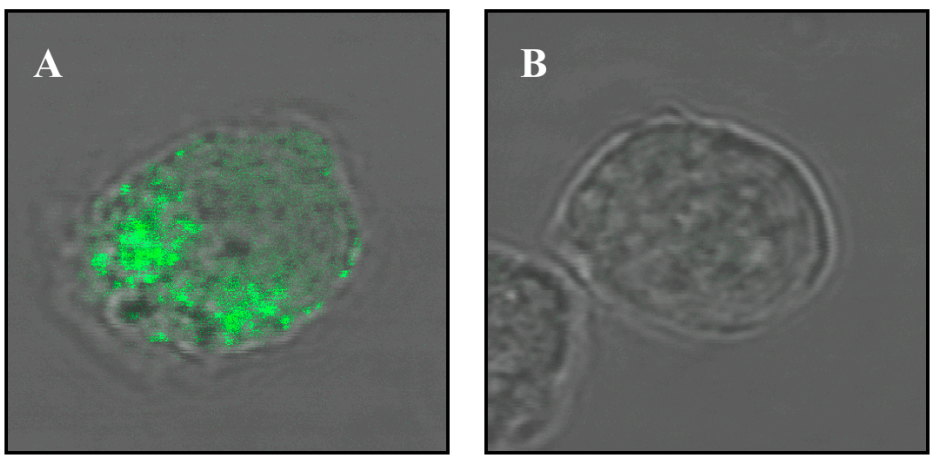


\title{
Chapter 3
}

\section{A role for CCR5 in dissemination of vaccinia virus in vivo}

\author{
Chapter 3 was published as \\ Rahbar R., Murooka T. and Fish E.N. A role for CCR5 in dissemination of vaccinia virus \\ in vivo. J Virol. 2009 Mar;83(5):2226-36. Epub 2008 Dec 10.
}

R.R. performed all experiments, analyzed the data and drafted the manuscript. 


\subsection{ABSTRACT}

In an earlier report, we provided evidence that expression of CCR5 by primary human $\mathrm{T}$ cells renders them permissive for vaccinia virus (VACV) replication. This may represent a mechanism for dissemination throughout the lymphatic system. To test this hypothesis, wild type $\mathrm{CCR}^{+/+}$and CCR5 null mice were challenged with VACV by intranasal inoculation. In time course studies using different infective doses of VACV, we identified viral replication in the lungs of both $\mathrm{CCR} 5^{+/+}$and $\mathrm{CCR} 5^{-/-}$mice, yet diminished viral loads in the spleens and brains of $\mathrm{CCR}^{-/-}$mice compared with $\mathrm{CCR} 5^{+/+}$ mice. Moreover, associated with VACV infection, we provide evidence for CD4+ and $\mathrm{CD} 8+\mathrm{T}$ as well as CD11c+ and F4/80+ cell infiltration into the lungs of CCR $5^{+/+}$but not $\mathrm{CCR}^{-/-}$mice, and show that the CCR5-expressing $\mathrm{T}$ cells harbor virus. We demonstrate that this CCR5-dependence is VACV-specific, since $\mathrm{CCR}^{-/-}$mice are as susceptible to intranasal influenza (A/WSN/33) infection as $\mathrm{CCR}^{+/+}$mice. In a final series of experiments we provide evidence that adoptive transfer of $\mathrm{CCR}^{+/+}$bone marrow leukocytes into $\mathrm{CCR}^{-/-}$mice restores VACV permissiveness, with evidence of lung and spleen infection. Taken together, our data suggest a novel role for CCR5 in VACV dissemination in vivo. 


\subsection{INTRODUCTION}

Vaccinia virus (VACV) is the best known member of the Orthopoxvirus genus of the Poxviridae, used for the vaccine strain that led to the global eradication of smallpox (193). VACV has a large double-stranded DNA genome of approximately $200 \mathrm{~kb}$, a cytoplasmic site of replication, many virus-encoded enzymes for transcription and DNA replication, and a complex morphogenic pathway that produces distinct forms of infectious virions from each infected cell (449). Variola, the causative agent of smallpox, killed more people than any other pathogen in the history of mankind (165). In 1980, the World Health Organization (WHO) certified that the world was free of smallpox, but the potential for variola to be used as a potential biological weapon has led to renewed interest in the pathogenesis of smallpox and the development of therapies (86). While variola has a narrow natural host range in humans, with non-human primates exhibiting susceptibility in a laboratory setting(93) and vaccinia exhibits a broader range of infectible hosts, both intranasal infections result in similar dissemination of virus: replication occurs initially in lymph nodes draining the upper respiratory tract, after which a transient viremia spreads virus to reticuloendothelial cells throughout the host. Notably, in variola alone, a second round of replication within these sites precedes a more intense secondary viremia and the subsequent clinical manifestations of the disease. Based on the pathogenic similarities, VACV presents as a suitable model for variola infection $(85,259,265-267,539)$.

Many poxviruses, including VACV, employ strategies to modulate chemokine activity, including virus-encoded chemokine-binding proteins (C23L/B29R encoded by VACV, G3R encoded by variola virus, DIL/H5R proteins encoded by cowpox virus)(17, 
$240,357,633)$ and receptor homologues (K2R homologue of a CXCR encoded by swinepox virus, Q2/3L homologue of a CCR encoded by caripoxvirus) (106, 353, 414, $466,612)$. The involvement of chemokine receptors in poxviral infection was suggested in studies utilizing the rabbit poxvirus, myxoma. Specifically, CCR5 was implicated in mediating cell target susceptibility to infection in BGMK cells (355), later shown to correlate with intracellular signaling $(314,416)$. In a recent publication we provided evidence that virus activation of CCR5 leads to the selective activation of distinct signaling pathways that are advantageous for the virus (536). We demonstrated that VACV infection in permissive cells is inhibited by siRNA knockdown of cell surface CCR5 expression and by the CCR5 antagonist, TAK-779. The importance of tyrosine phosphorylation of CCR5 was suggested by the observation that introduction of a CCR5 mutant, in which all the intracellular tyrosines are replaced by phenylalanines, effectively reduces VACV infection in permissive cells. Moreover, tyrosine-339 was implicated in CCR5 as the critical residue for mediating viral infection, since cells expressing CCR5.Y339F do not support viral replication. The cascade of events that leads to permissive phenotype of these cells includes phosphorylation activation of multiple signaling effectors: Jak-2, IRS-2, ERK1/ 2 and Grb2. These data were supported by findings that viral replication in permissive CCR5 expressing cells is blocked by Herbimycin A, and the Jak2 inhibitor, tyrophostin AG490, but not pertussis toxin. Viewed altogether, a critical role of post-entry events, specifically intracellular tyrosine phosphorylation events, was established in determining permissiveness of cells to VACV replication. Furthermore, evidence was provided that introduction of CCR5 in primary human T cells renders them permissive to VACV replication. Since permissive infection 
of $\mathrm{T}$ cells might represent a mechanism for VACV dissemination throughout the lymphatic system, we hypothesize that the absence of CCR5 may be protective against VACV infection in vivo.

Several mouse models of VACV infection that differ in mouse and viral strains, as well as routes of virus inoculation, have provided insights into the immunopathogenesis of VACV in vivo $(61,307,544)$. Herein, employing an intranasal inoculation route of VACV infection we provide supportive evidence for a role for CCR5 in VACV dissemination in vivo.

\subsection{RESULTS}

\subsubsection{CCR5 ${ }^{--}$mice are less susceptible to systemic VACV infection}

To investigate the contribution of CCR5 to the pathogenesis of VACV infection, $\mathrm{CCR}^{-/-}$and $\mathrm{CCR}^{+/+}$mice were either mock-infected or infected with $10^{4}, 10^{5}$ or $10^{6}$ p.f.u. of VACV strain WR by intranasal inoculation, and monitored daily for weight loss. Infection with $10^{4}$ p.f.u. of VACV resulted in approximately $8 \%$ weight loss in CCR $5^{+/+}$ but not $\mathrm{CCR}^{-/-}$mice (Figure 3.1A), whereas mock infection had no effect on all mice. When infected with $10^{5}$ or $10^{6}$ p.f.u. of VACV both $C C R 5^{+/+}$and CCR $5^{-/-}$mice exhibited weight loss, with a significantly greater weight loss in the $C C R 5^{+/+}$mice $(p<0.05)$. When infected with $10^{5}$ p.f.u. of VACV, CCR $5^{+/+}$mice exhibited approximately $14 \%$ weight loss, while $\mathrm{CCR}^{-/-}$lost approximately $7 \%$ of their body weight. At day 5 p.i., $\mathrm{CCR} 5^{+/+}$ mice infected with $10^{6}$ p.f.u. of VACV were euthanized since they had lost $20 \%$ of their original body weight, while $\mathrm{CCR}^{-/-}$mice reached $20 \%$ weight loss at day 7 (Figure $1 \mathrm{~A}$ ). 
We next examined the lungs, spleens and brain tissues of $\mathrm{CCR}^{-/-}$and $\mathrm{CCR} 5^{+/+}$ mice for evidence of VACV. In earlier studies, we provided evidence that VACV entry into cells is not mediated by CCR5, since virus enters both permissive and nonpermissive cells. A post-entry event in T cells, mediated by CCR5, determines whether viral replication ensues (536). In the present study, examination of viral titers in the lungs of $\mathrm{CCR}^{+/+}$mice infected with $10^{4}$ p.f.u. of $\mathrm{VACV}$ revealed a $>3$-fold log increase from $10^{4}$ to $\sim 1.2 \times 10^{7}$ p.f.u. by day 7 p.i., in contrast to the lungs of the CCR $5^{-/-}$mice, where viral titers increased by $<1 \log$, to $3.4 \times 10^{4}$ p.f.u. by day 7 p.i. (ANOVA indicates significant differences between all data points for $\mathrm{CCR}^{+/+}$and $\mathrm{CCR} 5^{-/-}$mice)(Figure 1B). Comparable viral titres have been identified in the trachea and lungs of mice post intranasal infection with VACV, with relatively low viral load in nasal washings (27). Accordingly, we focused our analyses on lung tissues. At 24 hrs p.i. we observe a log reduction in viral titre in the lungs of $\mathrm{CCR}^{-} \%^{-}$compared to the lungs of $\mathrm{CCR} 5^{+} \digamma^{+}$mice. Given our earlier data which suggest a role for CCR5 in viral permissivity (39), and the fact that $\mathrm{T}$ cells, resident monocytes and macrophages, together with other potential target cells types (e.g. fibroblasts) express CCR5, this reduced pathogenesis in the $\mathrm{CCR}^{-/}$mice was predicted. Notably, at the higher infective doses of $10^{5}$ and $10^{6}$ p.f.u. of VACV, viral titers measured in the lung tissues of $\mathrm{CCR}^{+/+}$and $\mathrm{CCR} 5^{-/-}$mice on days 1 , 3, 5 and 7 p.i. were comparable.

We next examined splenic viral titers, as a measure of viral dissemination from the primary site of infection. At the infective dose of $10^{4}$ p.f.u. of $\mathrm{VACV}, \mathrm{CCR} 5^{-/-}$mice showed no evidence of virus in splenocytes (virus titers $<120$ plaques/g tissue), in contrast to $\mathrm{CCR}^{+/+}$mice, where viral titers increased from day 1 to day 7 p.i. $(>750$ 
plaques/g tissue) (Figure 3.1B, ANOVA indicates significant differences at day 7 p.i. for splenic virus titers). At the higher infective dose of $10^{5}$ p.f.u. of VACV, viral titers in the spleens of $\mathrm{CCR}^{+/+}$mice were significantly higher than viral titers in the spleens of $\mathrm{CCR}^{-/-}$mice. A similar trend was observed on day 3 p.i. in the spleens of mice inoculated with $10^{6}$ p.f.u. of VACV, namely higher viral titers in the $\mathrm{CCR}^{+/+}$compared with the $\mathrm{CCR}^{-/-}$mice.

We observed a similar trend in viral titers in the brains of $\mathrm{CCR} 5^{+/+}$and $\mathrm{CCR} 5^{-/-}$ mice. Specifically, an intranasal inoculating dose of $10^{4}$ p.f.u. of VACV resulted in measurable virus in the brains of $\mathrm{CCR}^{+/+}$on days 3 and 7 p.i., in contrast to the $\mathrm{CCR} 5^{-/-}$ mice, where there was no detectable virus in their brains on day 3 p.i., with measurable, yet significantly lower viral titers on day 7 p.i. (Figure 3.1B). At the higher infecting doses of VACV, virus was detected in the brains of $\mathrm{CCR}^{+/+}$and $\mathrm{CCR}^{-/ /}$mice, albeit lower viral titers in the brains of the $\mathrm{CCR}^{-/-}$mice.

Viewed altogether, the data indicate that even when lung viral titers are comparable in $\mathrm{CCR}^{+/+}$and $\mathrm{CCR}^{-/ /}$mice, viral dissemination to the spleen and brains of $\mathrm{CCR}^{-/}$infected mice is reduced, compared with viral dissemination in the infected $\mathrm{CCR}^{+/+}$mice. 


\section{Figure 3.1}

\section{$\mathrm{CCR5}^{-/-}$mice are less susceptible to VACV infection}

Groups of female mice age 6-8 wks were either mock-infected (PBS) or infected with $10^{4}, 10^{5}$ or $10^{6}$ p.f.u. of VACV by intranasal inoculation. (A) Body weight was measured daily and values are recorded as the mean percent weight loss at the indicated time point compared to that of uninfected control mice \pm SE. $10^{4}$ p.f.u. $(n=10) ; 10^{5}$ p.f.u. $(n=12$ until day $8, n=7$ thereafter); $10^{6}$ p.f.u. ( $n=12$ until day $3, n=7$ thereafter).

$\mathrm{CCR}^{+/+}$, mock infected $\boldsymbol{\Delta}$; $\mathrm{CCR}^{+/+}, \mathrm{VACV}$ infected $\bullet$; $\mathrm{CCR}^{-/-} /$, mock-infected $\bullet$; $\mathrm{CCR}^{-/-}, \mathrm{VACV}$ infected $\square$.

Viral titers were measured in (B) lungs, spleens and brain of $\mathrm{CCR}^{+/+}$and $\mathrm{CCR} 5^{-/-}$mice $(n=10)$ at the indicated times p.i. with $10^{4}$ p.f.u. of VACV, as described in Materials and Methods. (C) Lungs of mock- or $10^{4}$ p.f.u. VACV- infected $\mathrm{CCR}^{+/+}$and $\mathrm{CCR} 5^{-/-}$mice were harvested on day 7 p.i., fixed in $2 \%$ paraformaldehyde and embedded in paraffin. 6 $\mu \mathrm{m}$ thick histological sections were prepared and stained with hematoxylin/eosin.

The dotted line indicates the lower level of detection for VACV.

*, $P<0.05 . \mathrm{ND}=$ Not Detected 
Figure 1
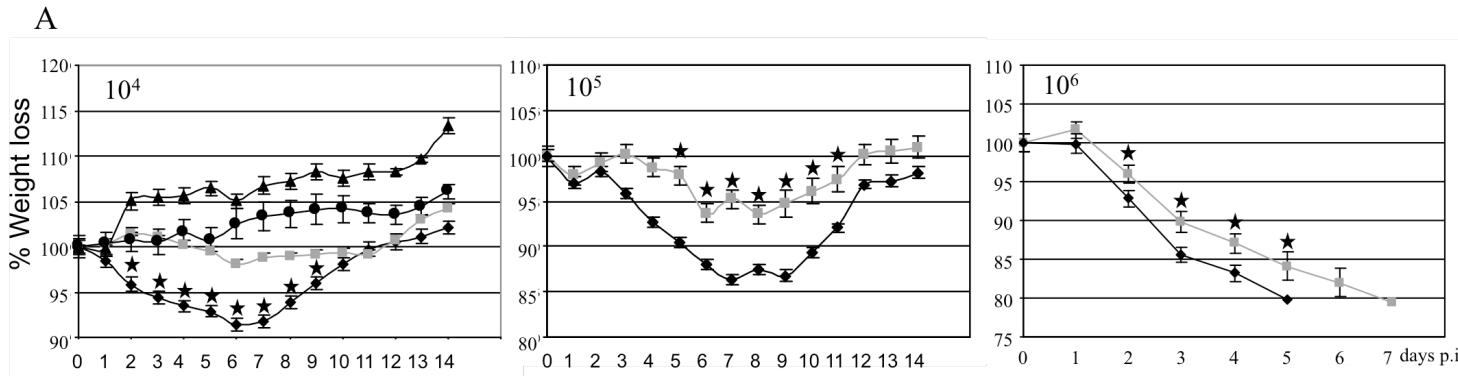

B
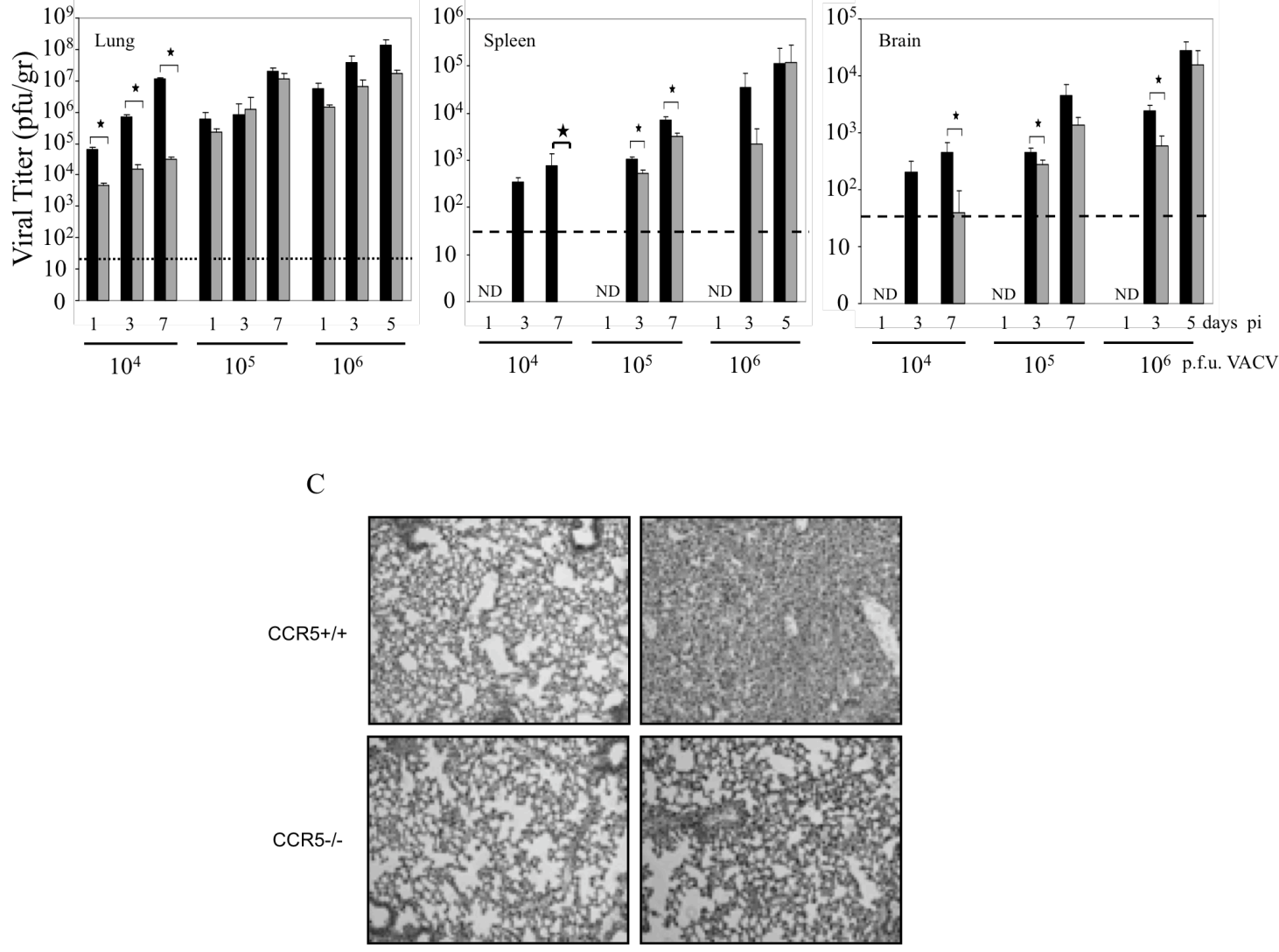

Naïve

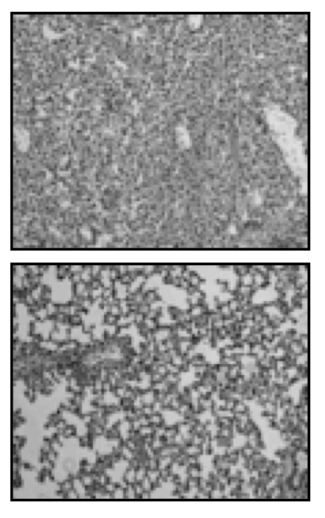

7 days p.i. 


\subsubsection{VACV infection of $\mathrm{CCR5}^{+/+}$but not $\mathrm{CCR5}^{-/-}$mice leads to infiltration of CD4+ and $C D 8+T$ cells into the lungs}

At the infective dose of $10^{4}$ p.f.u. of VACV, the lungs of $\mathrm{CCR}^{+/+}$mice exhibited inflammatory infiltrates and extensive histopathology by day 7 p.i., whereas no significant inflammatory cell influx or tissue destruction was observed in the lungs of $\mathrm{CCR}^{-/-}$mice (Figure 3.1C). In addition, at this infective dose, $\mathrm{CCR} 5^{+/+}$mice exhibited a significant increase in the number of viable cells accumulating in the bronchial alveolar lavage (BAL) in contrast to the BAL of $\mathrm{CCR} 5^{-/-}$mice: Cell numbers in the BAL from $\mathrm{CCR}^{+/+}$mice increased from 150,000 on day 0 to 320,000 by day 7 p.i., whereas cell counts in the BAL of CCR5 $5^{-/-}$mice increased from 140,000 to 207,000 over the same time period (Figure 3.2A). Moreover, in contrast to the increase in viral titers measured in the BAL from $\mathrm{CCR}^{+/+}$mice from day 1 to day 7 p.i., we identified a negligible increase in viral titers in the BAL from $\mathrm{CCR} 5^{-/-}$mice over the same time period (Figure 3.2B).

To examine the inflammatory response that is associated with intranasal VACV infection at $10^{4}$ p.f.u., the composition of immune cells in the lungs of VACV-infected $\mathrm{CCR}^{+/+}$and $\mathrm{CCR}^{-/-}$mice was characterized in time course studies. For this series of studies we employed EGFP-VACV. Notably, infection of CCR $5^{+/+}$mice with either $10^{4}$ p.f.u. of VACV WR or $10^{4}$ p.f.u. of EGFP-VACV results in comparable immune cell trafficking to BAL, mediastinal lymph nodes $(\mathrm{mLN})$ and spleens (Supplementary Figure 3.1). Employing flow cytometry to identify inflammatory cell types, we provide evidence for $\mathrm{CD} 4+$ and $\mathrm{CD} 8+\mathrm{T}$ cell $(\mathrm{CD} 3+)$ infiltration into the $\mathrm{BAL}$ of $\mathrm{CCR}^{+/+}$but not $\mathrm{CCR} 5^{-/-}$ mice during the course of VACV infection. Specifically, we observed a 5-fold increase in CD4+ and an approximately 8 - fold increase in CD8+ T cell influx in the BAL by day 7 
p.i. (Figure 3.2C, 2E). The modest influx of CD4+ and CD8+ T cells into the BAL of $\mathrm{CCR}^{-/-}$mice by day 1 p.i. was not sustained. Immunohistochemical staining of lung tissues from EGFP-VACV-infected $\mathrm{CCR} 5^{+/+}$and $\mathrm{CCR}^{-/-}$mice on day 7 p.i. for EGFPVACV, CD4 and CD8, revealed a greater number of $\mathrm{CD} 4+$ and $\mathrm{CD} 8+\mathrm{T}$ cell infiltrates in the lungs of $\mathrm{CCR}^{+/+}$versus $\mathrm{CCR} 5^{-/}$mice, with evidence of EGFP-VACV only in the CCR5 expressing CD4+ or CD8+ cells (Supplementary Figure 3.2). Employing flow cytometry to quantitate the number of CCR5 $5+$ or CCR5- CD4+ and CD8+ $\mathrm{T}$ cells that harbor EGFP-VACV in BAL, the data revealed that the increase in CD4+ and CD8+ T cell numbers in the BAL of VACV-infected $C C R 5^{+/+}$compared with VACV-infected $\mathrm{CCR}^{-/}$mice is attributable to those CD4+ and CD8+ T cells that express CCR5 (Figure 3.2D, F). At day 3 p.i. the increase in VACV infected CD4+ T cells (Figure 3.2D) and CD8+ T cells (Figure 3.2F) was approximately 5 -fold and 4-fold, respectively, in $\mathrm{CCR}^{+/+}$compared to $\mathrm{CCR}^{-/-}$mice. Interestingly, while total number of infected CD4+ and CD8+ T cells is approximately constant during the course of infection, the absolute numbers of $\mathrm{CD} 4+$ and $\mathrm{CD} 8+\mathrm{T}$ cells increase in $\mathrm{CCR}^{+/+}$mice. One consideration is that a percentage of infected $\mathrm{T}$ cells may undergo apoptosis post infection. Certainly, VACV undergoes abortive replication in DCs (181) and induces apoptotic cell death in both DCs and macrophages. In addition, we propose that there is a constant egress of infected $\mathrm{T}$ cells from the lungs, for systemic spread of virus. When infected with $10^{5}$ p.f.u. of VACV both $\mathrm{CCR}^{+/+}$and $\mathrm{CCR}^{-/-}$mice exhibited infiltration of $\mathrm{CD} 4+$ and $\mathrm{CD} 8+\mathrm{T}$ cells $(\mathrm{CD} 3+)$ into the BAL by day 7 p.i.. Notably, the number of infiltrating T cells was significantly higher in the $\mathrm{CCR}^{+/+}$mice: $2.5 \times 10^{5}$ versus $9 \times 10^{4}$ for $\mathrm{CD} 4+$, and $2 \times 10^{5}$ versus $6 \times 10^{4}$ for $\mathrm{CD}^{8}+\mathrm{T}$ cells (Supplementary Figure 3.3). 


\section{Figure 3.2}

Intranasal inoculation with VACV leads to an increase in BAL viral titer and influx of $\mathrm{CD4}^{+}$and $\mathrm{CD8}^{+} \mathrm{T}$ cells into the lungs of $\mathrm{CCR5}^{+/+}$but not $\mathrm{CCR5}^{-/-}$mice

Groups of female mice $(n=10), 6-8$ wks, were infected with $10^{4}$ p.f.u. of EGFP- VACV by intranasal inoculation. Mice were euthanized at the indicated times p.i. and their BAL collected as described in Materials and Methods. (A) Viable cell counts in the BAL of $\mathrm{CCR}^{+/+}(\mathbf{\square})$ and $\mathrm{CCR}^{-/-}(\square)$ were determined by trypan blue exclusion. Values are means \pm SE and are representative of two independent experiments. (B) Viral titers were measured in BAL fluid of $\mathrm{CCR}^{+/+}(\mathbf{a})$ and $\mathrm{CCR}^{-/-}$( $\square$ ) mice at the indicated times p.i. The dotted line indicates the lower level of detection for VACV.

(C) $\mathrm{CD}^{+}$and (E) $\mathrm{CD}^{+} \mathrm{T}$ cell counts were determined by flow cytometry, using PECy5-conjugated anti CD4/CD8 and APC-conjugated CD3 antibodies. ( $\boldsymbol{a}$ ) total $\mathrm{CD}^{+} / \mathrm{CD}^{+}$cell counts; ( $\left.\square\right) \mathrm{VACV}$-infected $\mathrm{CD} 4 / \mathrm{CD}^{+} \mathrm{T}$ cells, as determined by quantitation of EGFP positive cells as described in Materials and Methods. The data are partitioned as VACV-infected $\mathrm{CD}^{+}(\mathrm{D})$ and $\mathrm{CD}^{+}(\mathrm{F}) \mathrm{T}$ cells in the BAL of CCR $5^{+/+}$ and $\mathrm{CCR}^{-/-}$mice, recorded as the percent positive $\pm \mathrm{SE}$, representative of two independent experiments .

(a ) $\mathrm{CD}^{+} \mathrm{CCR}^{-} / \mathrm{CD}^{+} \mathrm{CCR}^{-} \mathrm{T}$ cell count in the BAL from $\mathrm{CCR} 5^{+/+}$mice;

(口) $\mathrm{CD}^{+} \mathrm{CCR}^{+} / \mathrm{CD}^{+} \mathrm{CCR}^{+} \mathrm{T}$ cell count in the BAL from $\mathrm{CCR} 5^{+/+}$mice;

( $\mathbf{\Delta}) \mathrm{CD}^{+} \mathrm{CCR}^{-} / \mathrm{CD}^{+} \mathrm{CCR}^{-} \mathrm{T}$ cell count in the BAL from $\mathrm{CCR}^{-/-}$mice.

$*, P<0.05 ; * *, P<0.01$ 
Figure 2
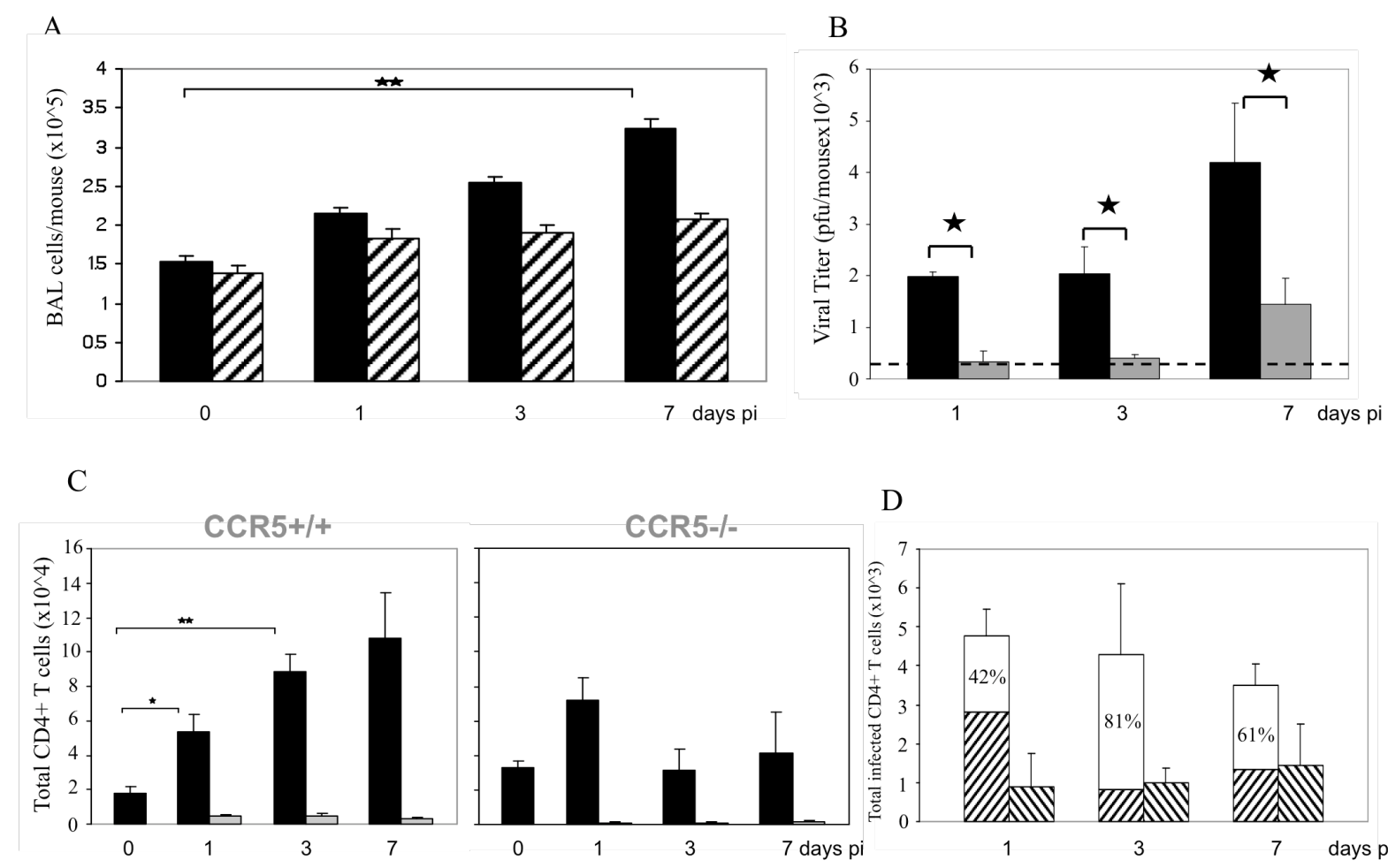

D
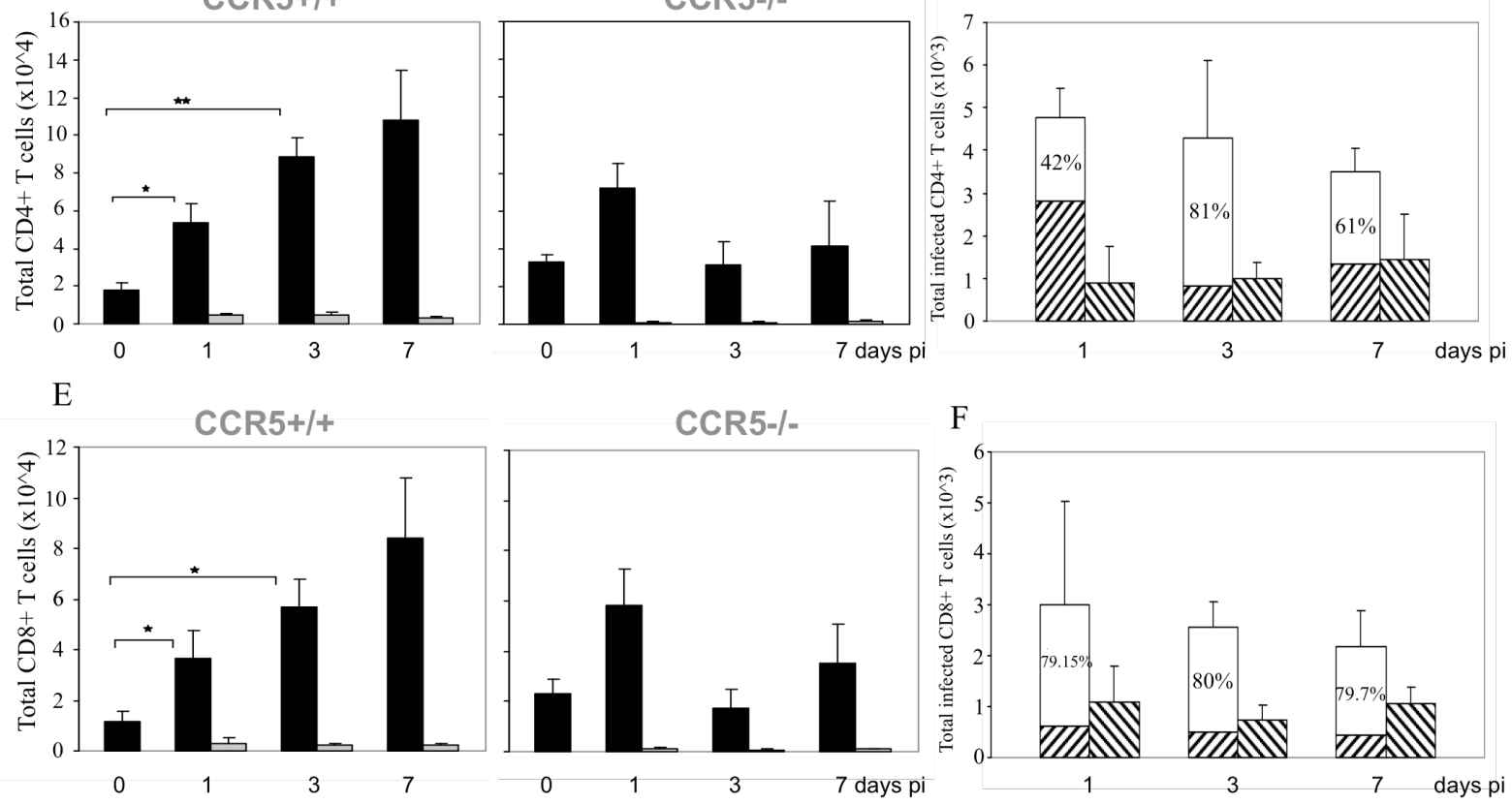


\subsubsection{Expression of CCR5 influences the $T$ cell population of secondary lymphoid organs}

Next, in time course studies during EGFP-VACV infection, we examined the CD4+ and CD8+ cell counts in the spleens and mLNs of $C C R 5^{+/+}$and $C C R 5^{-/-}$mice. Splenic CD4+ and CD8+ cells numbers decreased by 3 day p.i. for the $\mathrm{CCR}^{+/+}$mice, yet remained unchanged in the $\mathrm{CCR}^{-/-}$mice (Figure $3.3 \mathrm{~A}, \mathrm{~B}$ ). By day 7 p.i. the number of splenic CD4+ and CD8+ cells rebounded to the original number in the $\mathrm{CCR} 5^{+/+}$mice.

In contrast to the trend observed in the spleens of infected $\mathrm{CCR}^{+/+}$mice, there was a significant increase in the number of CD4+ and CD8 $+\mathrm{T}$ cells in their mLNs by day 3 p.i, which further increased by day 7 p.i. (Figure 3.3C,D). Specifically, for the $\mathrm{CCR}^{+/+}$mice , there was a 9- fold increase in CD4+ and an 8-fold increase in CD8+T cells by day 7 p.i. CD4+ and CD8+ T cell numbers remained unchanged, at basal levels, in the mLNs of infected $\mathrm{CCR}^{-/-}$mice. As measured by staining for CD62 and CD69, the number of activated CD4+ and CD8+ T cells (CD62- and CD69+) increased in the mLN of $\mathrm{CCR}^{+} /^{+}$but not $\mathrm{CCR} 5 \%^{-}$mice by day 7 p.i., reflective of antigen stimulation.

Specifically, in $\mathrm{CCR}^{+} /^{+}$mice the number of CD4+CD62- T cells was approximately 5fold greater than the same population in $\mathrm{CCR}^{-/}$mice $\left(5 \times 10^{5}\right.$ versus $\left.1 \times 10^{5}\right)$. Likewise, there was an approximate 8 -fold increase in $\mathrm{CD} 4+\mathrm{CD} 69+\mathrm{T}$ cells in the $\mathrm{CCR} 5^{+} \digamma^{+}$ compared to the $\mathrm{CCR}^{-} \%^{-}$mice $\left(1.6 \times 10^{5}\right.$ versus $\left.0.2 \times 10^{5}\right)$, and an increased number of $\mathrm{CD} 8+\mathrm{CD} 62-$ and $\mathrm{CD} 8+\mathrm{CD} 69+\mathrm{T}$ cells in the $\mathrm{CCR} 5^{+} /^{+}$compared to the $\mathrm{CCR} 5{ }^{\circ}$ mice ( $2.2 \times 10^{5}$ versus $0.5 \times 10^{5}$, and $4.5 \times 10^{4}$ versus $0.7 \times 10^{4}$, respectively)(Supplementary Figure 3.4). When infected with $10^{5}$ p.f.u. of VACV, both $\mathrm{CCR}^{+/+}$and $\mathrm{CCR} 5^{-/-}$mice showed increased numbers of CD4+ and CD8+ T cells $(\mathrm{CD} 3+)$ in their mLNs by day 7 
p.i., with a significantly greater number in the $\mathrm{CCR}^{+/+}$mice: $6.3 \times 10^{5}$ versus $2.7 \times 10^{5}$ for $\mathrm{CD} 4+$, and $6 \times 10^{5}$ versus $3 \times 10^{5}$ for $\mathrm{CD}^{8}+\mathrm{T}$ cells (Supplementary Figure 3.4 ).

\subsubsection{TAK-779 reduces VACV dissemination}

In a recent publication, data are provided that demonstrate that subcutaneous injection of TAK-779, a CCR5 antagonist, in a mouse model of asthma resulted in significantly diminished pulmonary inflammation. The analyses focused on BAL and lung tissue (45). Expression of CCR5 in total lung cell suspensions was reduced in the TAK-779 treated mice compared to untreated mice. Additionally, the number of immune cells in affected lungs, in mice treated with TAK-779, was also reduced. These findings are consistent with TAK-779 treatment disabling CCR5 signaling such that chemokinemediated cell trafficking is inhibited. Accordingly, to further examine the effects of CCR5 on VACV dissemination, $\mathrm{CCR}^{+/+}$mice were treated with $150 \mu \mathrm{g}$ of TAK-779 subcutaneously (770) one day prior to intranasal inoculation with VACV and thereafter daily for 7 days p.i. At day 7 p.i. mice were sacrificed, their lungs and spleens harvested and analyzed for evidence of virus infection. Notably, treatment with TAK-779 had no effect on lung viral titers, but specifically reduced VACV dissemination to the spleens. Specifically, lung viral titers for both untreated and TAK-779 treated mice were $10^{8}$ p.f.u./g tissue, whereas TAK-779 treatment resulted in reduced splenic virus titers from $1.2 \times 10^{3}$ p.f.u./g tissue in untreated mice to $4 \times 10^{2}$ p.f.u./g tissue in treated mice (Figure 3.4). 
Figure 3.3

VACV infection of $\mathrm{CCR5}^{+/+}$but not $\mathrm{CCR5}^{-/-}$mice influences the $\mathrm{T}$ cell responses in secondary lymphoid organs

Groups of female mice $(n=10), 6-8$ wks, were infected with $10^{4}$ p.f.u. of EGFP- VACV by intranasal inoculation. Mice were euthanized at the indicated times p.i. and $\mathrm{CD} 3+\mathrm{CD} 4+$ and $\mathrm{CD} 3+\mathrm{CD} 8+\mathrm{T}$ cell counts in the spleens $(\mathrm{A}, \mathrm{B})$ and $\mathrm{mLNs}(\mathrm{C}, \mathrm{D})$ were determined by flow cytometry, using PE-Cy5-conjugated anti CD4/CD8 and APCconjugated CD3 antibodies. ( $\mathbf{\square})$ total CD4+/CD8+.

Data are shown as mean cell counts $\pm \mathrm{SE}$ and are representative of two independent experiments. ${ }^{*}, P<0.05 ; * *, P<0.01$ 
Figure 3

A

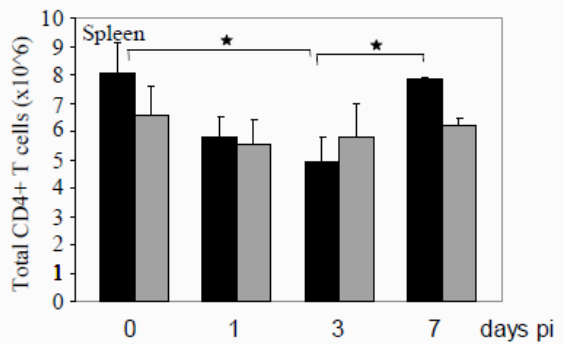

C

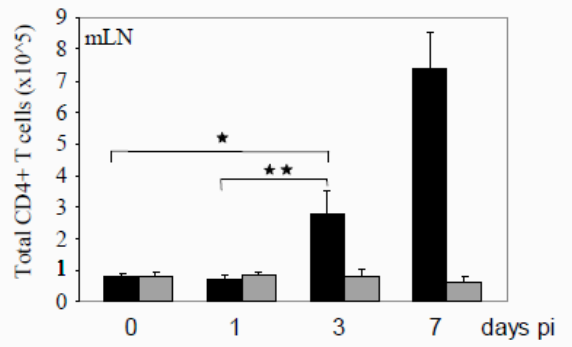

B

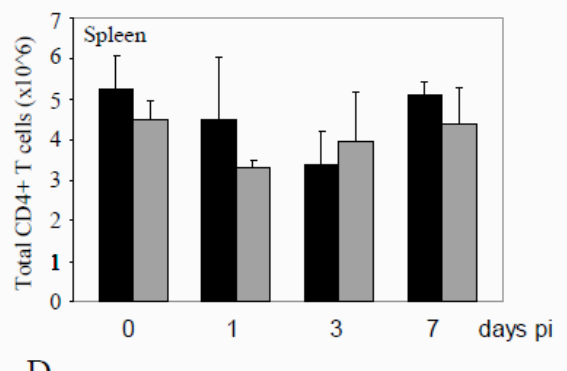

D

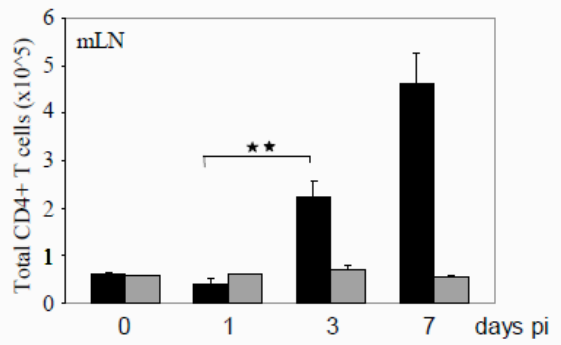




\section{Figure 3.4}

\section{TAK-779 reduces splenic VACV infection}

Female CCR5 ${ }^{+/+}$mice, 6-8 wk $(\mathrm{n}=10)$ were either left untreated $(\square)$ or received a subcutaneous injection of $150 \mu \mathrm{g}$ of TAK-779 ( $\square$ ) one day prior to and daily post intranasal inoculation with $10^{4}$ p.f.u. of VACV. On day 7 p.i. mice were sacrificed, and lung and splenic viral titers were determined. ${ }^{*}, P<0.05$

The dotted line indicates the lower level of detection for VACV. 
Figure 4
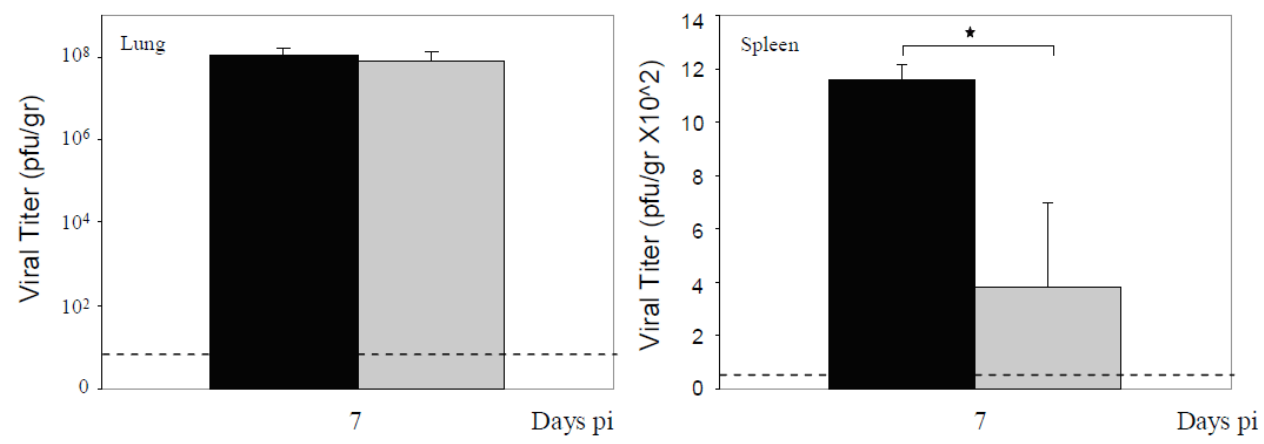


\subsubsection{Synchronous effects of VACV infection on antigen presenting cells in $\mathrm{CCR5}^{+/+}$ and $\mathrm{CCR5}^{-/-}$mice}

Circulating naïve $\mathrm{T}$ cells encounter antigen in secondary lymphoid organs spleen, LNs - presented by antigen-bearing CD11c + dendritic cells (DC). In the context of a pulmonary influenza infection, DCs in the $\mathrm{mLN}$ present virus-specific antigen to naïve $\mathrm{T}$ cells thereby invoking $\mathrm{T}$ cell activation and proliferation and subsequent egress. The data in Figure 5A demonstrate an increase in CD11c+ DCs by day 7 p.i. with VACV in the $\mathrm{mLN}$ of $\mathrm{CCR}^{+/+}$but not the $\mathrm{CCR} 5^{-/}$mice, consistent with the increase in $\mathrm{T}$ cell numbers observed in the $\mathrm{mLNs}$ of $\mathrm{CCR}^{+/+}$but not $\mathrm{CCR}^{-/ /}$mice (Figure 3.3C,D). By day 7 p.i., splenic DC numbers in the $\mathrm{CCR}^{+/+}$mice were elevated, yet remained unchanged in the $\mathrm{CCR}^{-/-}$mice (Figure 3.5B).

Similar to DCs, F4/80+ mature macrophages function as professional antigen presenting cells. Accordingly, in time course studies we examined the influence of VACV infection on $\mathrm{F} 4 / 80+$ cell counts in the $\mathrm{mLNs}$ and spleens of $\mathrm{CCR} 5^{+/+}$and $\mathrm{CCR} 5^{-/}$ mice. Consistent with the DC data, we provide evidence of an increase in $\mathrm{F} 4 / 80+$ cells by day 7 p.i. in the $\mathrm{mLNs}$ of $\mathrm{CCR}^{+/+}$but not $\mathrm{CCR}^{-/-}$mice (Figure 3.5C). Additionally, by day 7 p.i. splenic $\mathrm{F} 4 / 80+$ cell counts in the $\mathrm{CCR} 5^{+/+}$mice were elevated, yet remained unchanged in the $\mathrm{CCR}^{-/-}$mice (Figure 3.5D). Viewed altogether, we provide evidence that the higher viral loads in the lungs of $\mathrm{CCR} 5^{+/+}$mice results in increased mobilization of DCs and macrophages as antigen presenting cells. 


\section{Figure 3.5}

\section{VACV infection modulates CD11c+ dendritic cell and F4/80+ macrophage populations in the mLNs and spleens of $\mathrm{CCR5}^{+/+}$but not $\mathrm{CCR5}^{-/-}$mice}

$\mathrm{CD} 11 \mathrm{c}+$ and F4/80 cell infiltration into the $\operatorname{mLN}(\mathrm{A}, \mathrm{C})$ and spleen $(\mathrm{B}, \mathrm{D})$ of EGFPVACV- infected mice ( $10^{4}$ p.f.u.) was determined by flow cytometry, using APCconjugated anti CD11c and PE-Cy5-conjugated anti F4/80 antibodies antibodies. Data are presented as total cell number infiltrate of CD11c+/F4/80cells. ( $\boldsymbol{\square}$ ) total $\mathrm{CCR}^{+/+} \mathrm{CD} 11 \mathrm{c}+/ \mathrm{F} 4 / 80+,(\square)$ total $\mathrm{CCR} 5^{-/-} \mathrm{CD} 11 \mathrm{c}+/ \mathrm{F} 4 / 80+$. Data are shown as mean cell counts \pm SE and are representative of two independent experiments. $*, P<0.05 * *, P<$ 0.01 
Figure 5
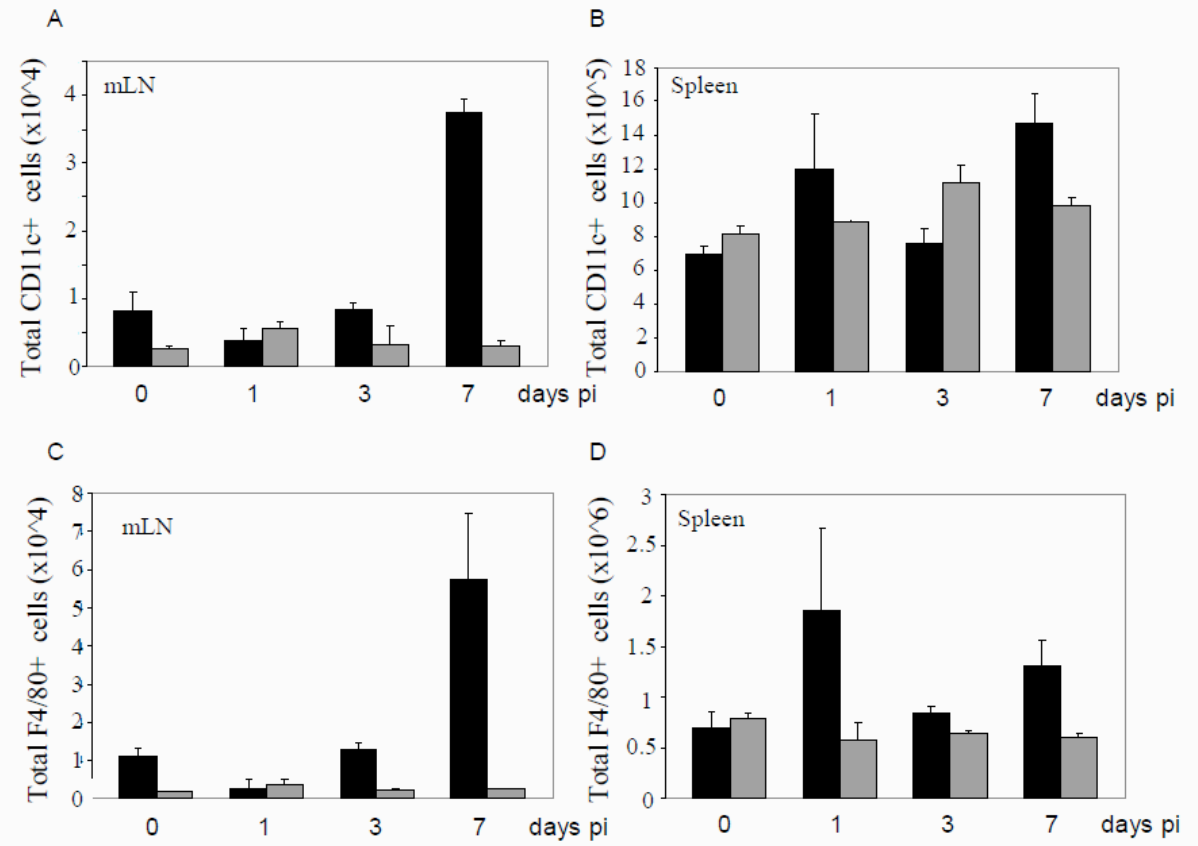


\subsubsection{CCR5 expression does not contribute to influenza A/WSN/33 infection}

To evaluate whether this CCR5-dependence for virus dissemination of a respiratory infection is VACV-specific, $\mathrm{CCR} 5^{+/+}$and $\mathrm{CCR} 5^{-/-}$mice were infected with 0.1 hemagglutinin unit (H.A.U.) of influenza A/WSN/33 virus via intranasal inoculation and monitored daily for symptoms of infection. As shown in Figure 3.6A, in time course studies, infection with this dose of influenza A/WSN/33 virus resulted in approximately $5 \%$ weight loss in both $\mathrm{CCR}^{+/+}$and $\mathrm{CCR} 5^{-/-}$mice. We next examined sections of lung tissues from infected mice for evidence of cellular infiltrates. We observed an increase in cellular infiltrates into the lungs of both $\mathrm{CCR} 5^{+/+}$and $\mathrm{CCR} 5^{-/-}$mice by days 3 and 6 p.i. (Figure 3.6B). In addition, comparable numbers of CD4+, CD8+, CD11c+ and F4/80+ cells were detected in the spleens and mLNs of $\mathrm{CCR} 5^{+/+}$and $\mathrm{CCR} 5^{-/-}$infected mice on days 3 and 6 p.i. (Figure 3.6C).

We next examined the effects of higher inoculating doses of influenza A/WSN/33 virus in $\mathrm{CCR} 5^{+/+}$and $\mathrm{CCR} 5^{-/-}$mice. $\mathrm{CCR} 5^{+/+}$and $\mathrm{CCR} 5^{-/-}$mice were infected with 1 (Supplementary Figure 3.5) and 5 H.A.U. (Supplementary Figure 3.6) of influenza A/WSN/33 virus via intranasal inoculation and monitored daily for symptoms of infection. In time course studies both $\mathrm{CCR} 5^{+/+}$and $\mathrm{CCR} 5^{-/-}$mice exhibited weight loss, with evidence of greater weight loss in $\mathrm{CCR}^{-/-}$mice. On days 9 and 10 p.i. with 1 H.A.U. of virus, $\mathrm{CCR} 5^{-/-}$and $\mathrm{CCR} 5^{+/+}$mice were euthanized, respectively, since they had lost $>20 \%$ of their original body weight. Similarly, all mice infected with 5 H.A.U. were sacrificed on 5 day p.i. because of weight loss. Examination of lung tissue thin sections for evidence of cellular infiltrates revealed extensive cellular infiltrates into the lungs of both $\mathrm{CCR}^{+/+}$and $\mathrm{CCR}^{-/-}$mice infected with 1 and 5 H.A.U. of virus. Flow cytometry 
analysis provided evidence for higher $\mathrm{CD} 4+, \mathrm{CD} 8+, \mathrm{CD} 11 \mathrm{c}+$ and $\mathrm{F} 4 / 80+$ cell numbers in the BAL of $\mathrm{CCR}^{-/-}$mice compared to $\mathrm{CCR} 5^{+/+}$mice, when infected with 5 H.A.U. of virus, in agreement with previously reported data (29), and comparable infiltrating cell numbers by days 3 and 6 p.i. when infected with 1 H.A.U. of the virus (Supplementary Figure 5,6). Similar numbers of CD4+, CD8+, CD11c+ and F4/80+ cells were observed in the spleens and mLNs of $\mathrm{CCR} 5^{+/+}$and $\mathrm{CCR} 5^{-/-}$infected mice after infection with either dose of influenza A/WSN/33 virus (Supplementary Figure 3.5,6). 


\section{Figure 3.6}

\section{$\mathrm{CCR5}^{-/-}$mice are permissive for influenza A/WSN/33 infection}

A) Groups of female mice $(n=6), 6-8$ wks, were infected with 0.1 H.A.U. influenza A/WSN/33 virus and body weight measured daily. Body weight loss was expressed as the mean percent weight loss of animals at the indicated time points compared to that of controls \pm SE. (- $) \mathrm{CCR}^{+/+}$; $\bullet$ ), $\mathrm{CCR}^{-/-}$(B) Lungs of mock- and influenza A/WSN/33infected $\mathrm{CCR}^{+/+}$and $\mathrm{CCR} 5^{-/-}$mice harvested on days 3 and 6 p.i. were fixed in $2 \%$ paraformaldehyde and embedded in paraffin. $6 \mu \mathrm{m}$ thick histological sections were prepared and stained with hematoxylin and eosin. CD3+CD4+ and CD3+CD8+, CD11c+ and F4/80+ cells present in BAL, spleens and mLNs of influenza A/WSN/33 infected $\mathrm{CCR}^{+/+}$and $\mathrm{CCR}^{-/-}$mice were quantitated by flow cytometry on days 3 (C) and 6 (D) p.i.. Cells were stained with PE-Cy5-conjugated anti- CD4, CD8, F4/80 and APCconjugated CD11c and CD3 antibodies.

( ) $\mathrm{CCR}^{+/+} ;(\square), \mathrm{CCR}^{-/-}$. Data are presented as mean cell counts $\pm \mathrm{SE}$ and are representative of two independent experiments. 
Figure 6

A

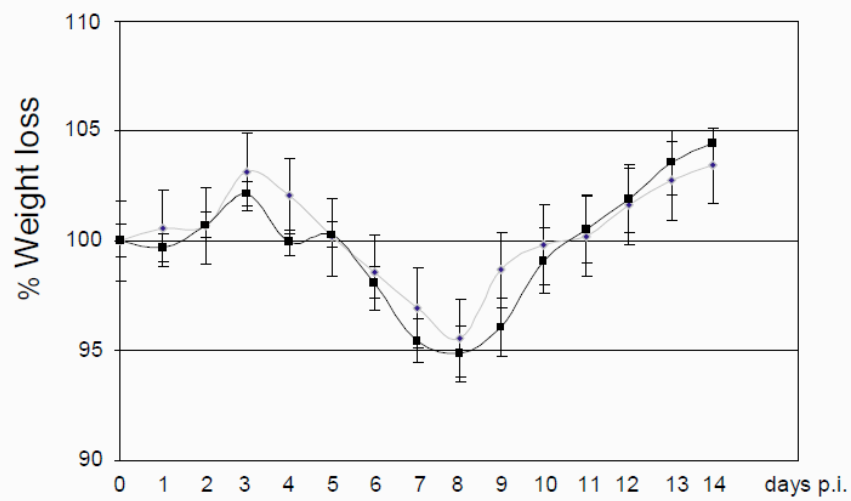

B

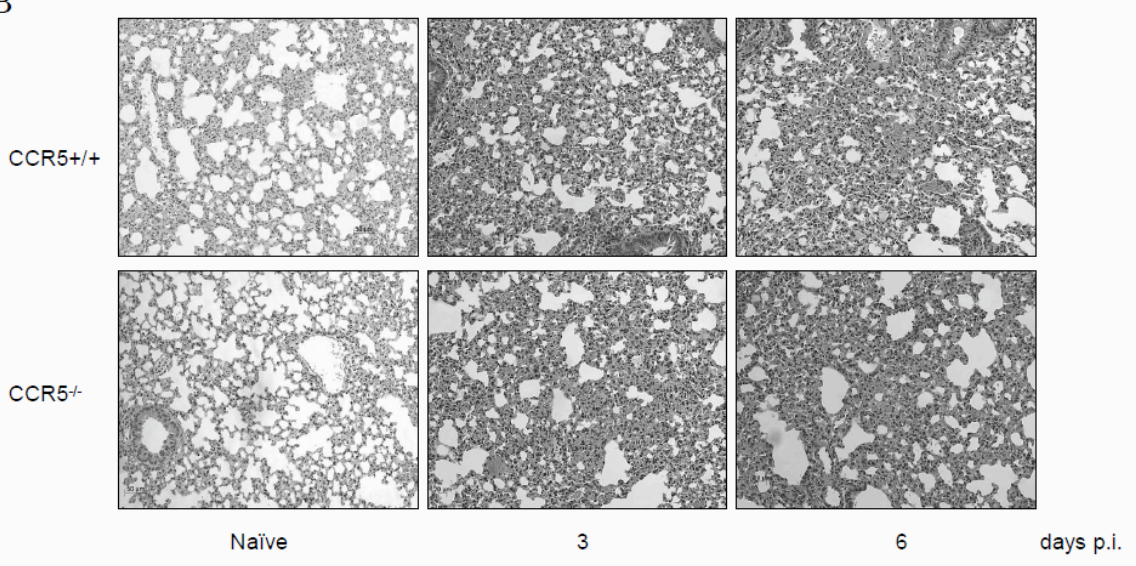

C

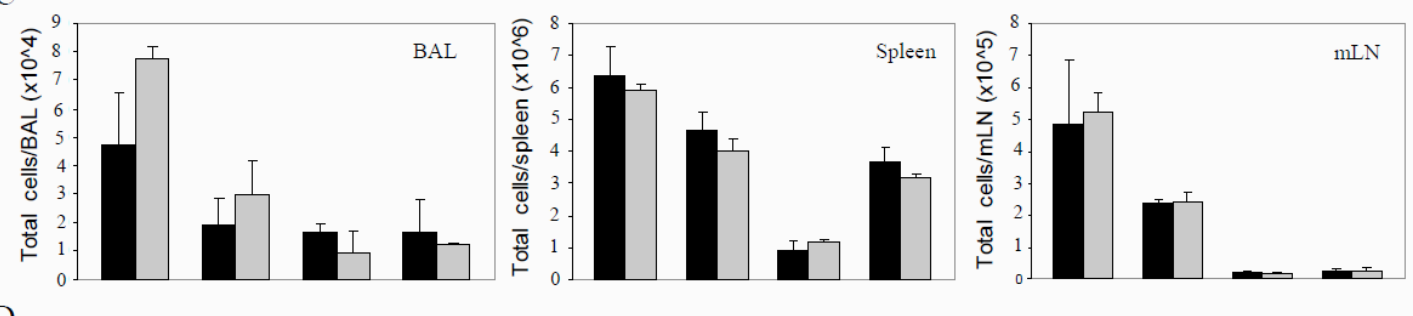

D
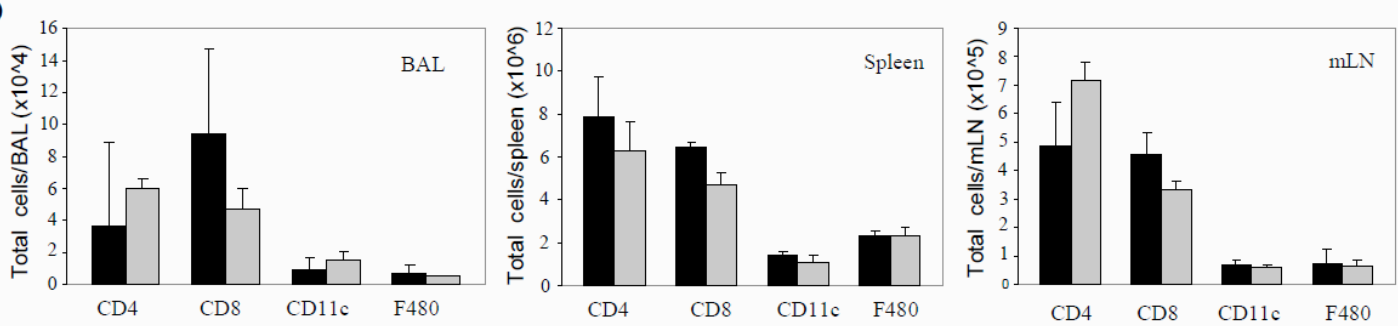


\subsubsection{Adoptive transfer of $\mathrm{CCR5}^{+/+}$leukocytes into $\mathrm{CCR5}^{-/-}$mice restores susceptibility to VACV infection}

To confirm that CCR5 expression contributes to the pathogenesis of VACV infection in vivo, we adoptively transferred bone marrow derived leukocytes from $\mathrm{CCR}^{+/+}$donor to both $\mathrm{CCR}^{-/-}$and $\mathrm{CCR}^{+/+}$recipient mice. $\mathrm{CCR} 5^{+/+}$and $\mathrm{CCR} 5^{-/-}$mice were irradiated with a sub-lethal dose of $1100 \mathrm{cGy}$ and, 4 hours post-irradiation, received $10^{6}$ hematopoietic leukocytes from $\mathrm{CCR}^{+/+}$donor mice. 2 weeks post-transplantation, all mice were infected with $10^{4}$ p.f.u. of VACV by intranasal inoculation. Mice were sacrificed at the indicated times p.i., at which times BAL was extracted and lungs, mLNs and spleens harvested.

Adoptive transfer of bone marrow derived hematopoietic leukocytes from $\mathrm{CCR}^{+/+}$mice into $\mathrm{CCR} 5^{-/-}$mice partially restored susceptibility to VACV-infectivity, as measured by viral titers in lung, BAL and spleens, and corroborated by the extent of CD4+ and CD8+ cell infiltrates into the BAL (Figure 3.7). Specifically, the data in Figure $6 \mathrm{~A}$ indicate that there were similar trends of increasing viral titers in the lungs, BAL and spleens of both $\mathrm{CCR}^{+/+}$and $\mathrm{CCR} 5^{-/-}$mice that received donor $\mathrm{CCR} 5^{+/+}$ leukocytes, albeit lower overall titers in the $\mathrm{CCR} 5^{-/-}$recipients. Furthermore, by day 7 p.i. there was a 4-fold increase in CD4+ and an 8-fold increase in CD8+ T cell infiltrates in the BAL of $\mathrm{CCR}^{-/-}$leukocyte recipients, compared with a 4-fold and 6-fold increase in $\mathrm{CD}^{+}$and $\mathrm{CD}^{+} \mathrm{T}$ cell infiltrates in the BAL of the $\mathrm{CCR} 5^{+/+}$recipients (Figure $6 \mathrm{~B}, \mathrm{C}$ ). Interestingly, when comparing the $\mathrm{CCR} 5^{-/-}$mice with $\mathrm{CCR}^{-/-}$mice that received donor $\mathrm{CCR}^{+/+}$leukocytes, the latter displayed a significant increase in the number of VACVinfected CD4+ and CD8+ cells. The majority of these infected cells were CCR5 positive. 
Unlike the $\mathrm{CCR} 5^{-/-}$mice, the $\mathrm{CCR} 5^{-/-}$mice that received donor $\mathrm{CCR} 5^{+/+}$leukocytes had similar CD4+ and CD8+ $\mathrm{T}$ cell numbers to the $\mathrm{CCR}^{+/+}$mice in their spleens (Figure 6D,E) and mLNs (Figure 3.7F,G).

The number of $\mathrm{CD} 11 \mathrm{c}+$ cells increased significantly by day 3 p.i. in the BAL (Figure 3.7H), spleens (Figure 3.7I) and $\mathrm{mLNs}$ (Figure 3.7J) of recipient $\mathrm{CCR}^{+/+}$mice, and similar trends were observed in the BAL and $\mathrm{mLNs}$ of recipient $\mathrm{CCR} 5^{-/ /}$mice that received donor $\mathrm{CCR}^{+/+}$leukocytes. This increase in $\mathrm{CD} 11 \mathrm{c}+$ cells was not observed in the spleens of the $\mathrm{CCR}^{-/ /}$mice that received donor $\mathrm{CCR} 5^{+/+}$leukocytes (Figure 3.7I).

In other analyses, we examined F4/80+ cell counts in the BAL, spleens and mLNs of $\mathrm{CCR}^{+/+}$and $\mathrm{CCR}^{-/ /}$recipient mice, at different times p.i. The results in Figure 6 reveal that adoptive transfer of $\mathrm{CCR} 5^{+/+}$leukocytes into $\mathrm{CCR} 5^{+/+}$and $\mathrm{CCR} 5^{-/ .}$mice resulted in similar levels of F4/80+ cells in their BAL (Figure 3.7K), spleens (Figure 3.7L) and mLNs (Figure 3.7M).

Viewed altogether, the data strongly support a role for the donor $\mathrm{CCR}^{+/+}$ leukocytes mediating permissiveness to VACV infection and dissemination. 


\section{Figure 3.7}

\section{Adoptive transfer of $\mathrm{CCR5}^{+/+}$leukocytes into $\mathrm{CCR5}^{-/-}$mice restores permissiveness to VACV infection}

Hematopoietic leukocytes from $\mathrm{CCR}^{+} /^{+}$mice were isolated and transplanted into irradiated $\mathrm{CCR}^{+} \Gamma^{+}$and $\mathrm{CCR} 5 \%$ mice as described in Materials and Methods. After two weeks, transplanted mice were either mock- or EGFP-VACV-infected ( $10^{4}$ p.f.u). (A) At the indicated times p.i., mice were euthanized and VACV titers were determined in lungs, BAL and spleens of recipient $\mathrm{CCR}^{+} \Gamma^{+}(-)$and $\left.\mathrm{CCR} 5^{-} \Gamma^{-}\right)$mice. The data are representative of two independent experiments. The dotted line indicates the lower level of detection for VACV. ND= Not Detected. Single cell suspensions from the BAL, spleen and $\mathrm{mLN}$ of recipient $\mathrm{CCR}^{+} \digamma^{+}(\boldsymbol{\square})$ and $\mathrm{CCR} 5{ }^{-} \digamma(\square)$ mice were obtained and stained with PE-Cy5-conjugated anti- CD4/ CD8/F480 and APC-conjugated anti$\mathrm{CD} 3 / \mathrm{CD} 11 \mathrm{c}$ antibodies at the indicated time points. Values are the mean $(\mathrm{B}, \mathrm{D}, \mathrm{F}) \mathrm{CD} 4^{+}$ and $(\mathrm{C}, \mathrm{E}, \mathrm{G}) \mathrm{CD}^{+} \mathrm{T}$ cell, $(\mathrm{H}, \mathrm{I}, \mathrm{J}) \mathrm{CD} 11 \mathrm{c}^{+}$and $(\mathrm{K}, \mathrm{L}, \mathrm{M}) \mathrm{F} 4 / 80^{+}$cell counts, $\pm \mathrm{SE}$ of two independent experiments. ( $\square$ ) total VACV-infected $\mathrm{CCR} 5^{+} /^{+} \mathrm{CD}^{+} / \mathrm{CD}^{+}$cells, ( $\square$ ) total VACV-infected CCR5 / CD4+/ CD8+/CD11c+/F4/80+cells. ${ }^{*}, P<0.05 ; * *, P<$ 0.01 
Figure 7
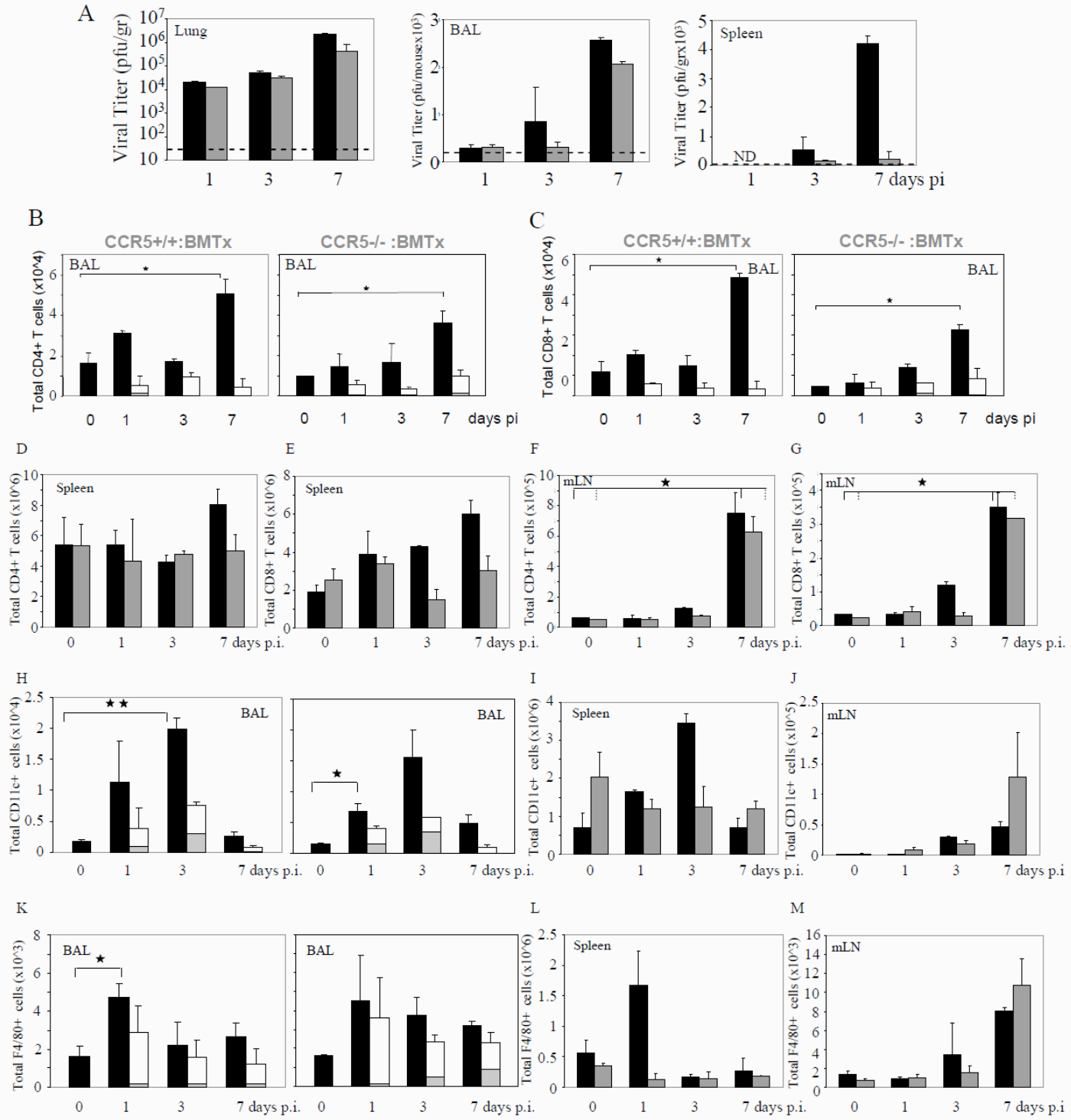


\subsection{DISCUSSION}

In an effort to recapitulate the human disease, we have employed VACV as the surrogate for variola virus, and the respiratory tract as the site of virus inoculation (189, 690, 748). The pathogenesis of VACV strain WR infection after intranasal inoculation is extensive respiratory infection followed by viremia leading to systemic infection with considerable lethality at high doses of virus. The details of viral dissemination and pathogenesis are not well understood. The rapid systemic infection with WR is accompanied by dissemination of the virus to the brain and visceral tissue, including spleen. Infection at non-lethal doses invokes immune cell infiltration to the lungs, and the virus is cleared by 15 days p.i.

Herein we provide an analysis of the cellular immune response that accompanies intranasal VACV infection and demonstrate the importance of CCR5 expression for viral dissemination in a mouse model of the disease. VACV undergoes abortive replication in DCs (181) and induces apoptotic cell death in both DCs and macrophages $(181,293)$, whereas productive infection occurs in activated, but not resting T cells (115). Indeed, following infection of mice with $\mathrm{VACV}$, a robust $\mathrm{T}$ cell response is elicited, associated with activated VACV-specific CD8+ T cells and CD4+ T cells $(256,544)$.

In activated T cells, susceptibility to infection is associated with the expression of a binding receptor that is induced de novo upon $\mathrm{T}$ cell activation $(115,227)$. When human peripheral blood T cells are activated with phytohemagglutinin and IL-12 (305), we observe the inducible de novo ectopic expression of CCR5 $(464,465)$. We speculate that inducible CCR5 expression upon $\mathrm{T}$ cell activation may render cells permissive to 
VACV replication. It has been suggested that the productive infection of a subpopulation of activated $\mathrm{T}$ cells may account for dissemination of the virus away from local sites of inoculation (50), herein supported by our data.

We provide several lines of evidence that suggest that trafficking of CD4+ and CD8+ T cells expressing CCR5 to the VACV-infected mouse lung is critical for subsequent dissemination of the virus. First, $\mathrm{VACV}$ inoculated $\mathrm{CCR} 5^{-/-}$mice displayed markedly reduced accumulation of CD4+ and CD8+ T cells in their BAL. Second, the majority of VACV infected CD4+ and CD8+ T lymphocytes in $\mathrm{BAL}$ of $\mathrm{CCR} 5^{+/+}$were CCR5-positive. Third, at the lower infecting doses of VACV there were reduced levels of virus in the spleens and brains of $\mathrm{CCR} 5^{-/-}$mice, compared with $\mathrm{CCR} 5^{+/+}$mice. When bone marrow leukocytes from $\mathrm{CCR} 5^{+/+}$mice were adoptively transferred into $\mathrm{CCR} 5^{-/-}$ mice, we observed a greater leukocyte accumulation in the BAL of transplanted $\mathrm{CCR} 5^{-/-}$ mice post VACV infection, and this coincided with viral dissemination to the spleen. Notably, although VACV-infected DCs and macrophages were identified in the lungs of infected mice, examination of the mLNs and spleens of mice p.i. showed no evidence of infected DCs or macrophages, from which we infer that these immune cells are not associated with harboring virus for viral dissemination. When infected with a higher dose of VACV, $\mathrm{CCR} 5^{-/}$mice consistently exhibited a level of resistance to infection: infected with $10^{5}$ p.f.u. of VACV, $\mathrm{CCR}^{+/+}$mice lost approximately $14 \%$ of their body weight by day 7 p.i., whereas $\mathrm{CCR}^{-/-}$mice lost approximately $7 \%$ of their body weight. Infected with $10^{6}$ p.f.u. of $\mathrm{VACV}, \mathrm{CCR}^{+/+}$mice lost approximately $20 \%$ of their body weight by day 5 p.i., whereas $\mathrm{CCR}^{-/ /}$mice reached $20 \%$ weight loss by day 7 p.i. Animal ethics guidelines require euthanization of mice at $20 \%$ loss of body weight, therefore all mice 
were sacrificed when they reached this weight loss. Accordingly, we are unable to determine whether the absence of CCR5 influences lethality or mortality rates at this high inoculating dose of VACV. Nevertheless, a delay of 2 days in disease progression is notable given the overwhelming viral inoculating dose. In addition, viral titers in the spleens and brains of the $\mathrm{CCR} 5^{-/}$mice were reduced by more than two-fold when infected with $10^{5}$ p.f.u. By day 3 p.i., viral titers in the spleens of the CCR $5^{-/}$mice were reduced by more than a log-fold, and by approximately four fold in their brains, compared to titers in the $\mathrm{CCR} 5^{+/+}$mice. At the higher infecting doses of VACV $\left(10^{5}\right.$ and $10^{6}$ p.f.u.), lung viral titers were indistinguishable between $\mathrm{CCR}^{+/+}$and $\mathrm{CCR} 5^{-/-}$mice, yet VACV dissemination to the spleens and brains of $\mathrm{CCR} 5^{-}$mice was clearly reduced compared with $\mathrm{CCR} 5^{+/+}$mice, in support of a role for CCR5 in virus dissemination.

Administration of TAK-779, a CCR5 antagonist, reduced viral dissemination to the spleens of $\mathrm{CCR}^{+/+}$mice, with no effect on viral lung titers. TAK-779 inhibits the migration of leukocytes, including T cells, and abrogates CCR5-inducible signaling in vivo (770). The dose of TAK-779 administered to the $\mathrm{CCR} 5^{+/+}$mice may have been insufficient to inhibit all expressed receptors (CCR5), which may have allowed for residual viral replication in the lung $\mathrm{T}$ cells. This would contrast with the $\mathrm{CCR} 5^{-/-}$mouse $\mathrm{T}$ cells, where no virus replication would occur. In addition, given that VACV infects cells of different lineages, including those that may not express CCR5, we might expect viral replication to be unaffected in some cell types. We speculate that a TAK-779 mediated reduction of CCR5-positive $\mathrm{T}$ cell trafficking to the primary site of infection, the lungs, together with the TAK-779 inhibition of CCR5-mediated signaling in a percentage of these T cells, required for VACV replication (24), may be reflected in the 
subsequent reduction of VACV dissemination to the spleen. Taken together, these data further support our hypothesis that CCR5 is important for VACV dissemination in vivo.

The role of CCR5 in viral pathogenesis is virus-specific. In a mouse model of West Nile Virus infection, expression of CCR5 is crucial for viral clearance(227). The human immunodeficiency virus (HIV) utilizes CCR5 as a co-receptor to mediate virus entry into target cells(383). In our studies, intranasal infection with influenza virus A/WSN/33 resulted in similar symptoms of disease in all mice, namely weight loss and comparable influx of CD4+, CD8+, CD11c+ and $\mathrm{F} 4 / 80+$ cells in $\mathrm{CCR}^{-/-}$and $\mathrm{CCR}^{+/+}$ mice, in the different tissue compartments examined. These results are consistent with earlier studies that have reported that CCR5 does not play a role in influenza A pathogenesis (734). Infection of alveolar epithelial cells with the mouse-adapted influenza A virus strain PR/8, strongly induces the release of monocyte chemoattractants CCL2 and CCL5, followed by a strong monocyte transepithelial migration. This monocytic response is strictly dependent on CCR2, but not CCR5 expression $(158,270)$. In fact, when infected with influenza A virus, $\mathrm{CCR}^{-/-}$mice exhibited a hyperinflammatory response, expressed as increased pulmonary inflammation and higher mortality (158). In a murine model of colitis, CCR5-deficient mice showed increased infiltration of CD4+ and NK1.1+ lymphocytes, along with a decrease in Th1 and an increase in Th2 cytokine expression (25), providing additional evidence that the absence of CCR5 expression per se does not prevent immune cell trafficking to a site of infection or inflammation. Of note, none of these studies address the heightened immune response in the $\mathrm{CCR} 5^{-/}$mice.

Our data clearly show that the adoptive transfer of bone marrow derived $\mathrm{CCR} 5^{+/+}$ leukocytes into $\mathrm{CCR} 5^{-/}$mice partially rescued the permissive phenotype to VACV. 
Specifically, we observed increased infiltration of CD4+ and CD8 $+\mathrm{T}$ cells as well as $\mathrm{CD} 11 \mathrm{c}+$ and $\mathrm{F} 4 / 80+$ cells into the lungs, and increased VACV dissemination into the spleens, of transplanted $\mathrm{CCR}^{-/-}$mice.

Despite the reported redundancy associated with some chemokine - receptor interactions $(45,347,405,524,734)$, herein we provide evidence that CCR5 is important for the migration of $\mathrm{T}$ cells into affected lungs following intranasal VACV infection.

Furthermore, the data demonstrate that CCR5 expression in T cells contributes to dissemination of VACV beyond the lung tissue. The data suggest that the role of CCR5 in VACV infection is not redundant and we infer that CCR5 may be required for systemic VACV infection in vivo.

\subsection{MATERIALS AND METHODS}

\subsubsection{Animals}

$\mathrm{CCR}^{-/-}$mice (B6;129P2-Ccr5tm1Kuz) and mice from their genetically matched mouse strain were purchased from the Jackson Laboratory and housed in microisolated cages in the animal colony at the Canadian Blood Services Building and the Ontario Cancer Institute, the University Health Network. All experiments were performed using female mice, 6-8 weeks of age. Mouse studies were performed in an animal biosafety level two facility under a protocol approved by the University Health Network Animal Care Committee. 


\subsubsection{Viruses}

VACV strain Western Reserve (WR) was a gift from Grant McFadden (University of Florida). A4L-EGFP-VACV (EGFP-VACV) (EGFP enhanced green fluorescent protein) was a gift from Dr. Geoffrey Smith (Imperial College of London) (112). Infection with either VACV WR or EGFP-VACV results in comparable infection and immune cell trafficking into bronchial alveolar lavage (BAL), mediastinal lymph nodes $(\mathrm{mLN})$ and spleens of infected mice (Supplementary Figure 1). VACV was purified as described previously (536). Briefly, virus was grown in HeLa cells and purified by sedimentation through a $36 \%$ sucrose cushion, and the number of virus particles determined by optical density measurement at $260 \mathrm{~nm}\left(1 \mathrm{U}=1.2 \times 10^{10}\right.$ particles $)$. The particle: p.f.u. ratio of VACV (WR) and EGFP-VACV was estimated to be 40:1 and $50: 1$, respectively.

\subsubsection{Virus infection}

All viral infection studies were performed in a biosafety level 2 room. Mice were anesthetized by intraperitoneal injection with $50 \mu \mathrm{l}$ of ketamine $(50 \mathrm{mg} / \mathrm{kg}) / \mathrm{xylazine}(2.5$ $\mathrm{mg} / \mathrm{kg}$ ) diluted in PBS. Immediately, mice received an intranasal inoculation of $10^{4}$ p.f.u. of either VACV WR or EGFP-VACV suspended in $20 \mu 1$ of sterile PBS. Control mice received only sterile PBS (mock infection). VACV (or PBS alone) was instilled into the nares, and mice were observed until the virus was inhaled. Mice were monitored daily for any symptoms of disease and were weighed daily during the course of the experiment. 


\subsubsection{Tissue isolation and viral titration}

Mice were sacrificed on days $0,1,3,7$ post-infection (p.i.), at which times lungs, brains, $\mathrm{mLN}$ and spleens were removed and bronchoalveolar lavage (BAL) fluid extracted for the indicated histology, viral titers and molecular analysis. Samples denoted for histology were either paraformaldehyde fixed and embedded in paraffin, or embedded in HistoPrep $^{\mathrm{TM}}$ (Fisher Scientific), submerged in ice-cold isopentane, then snap frozen in liquid nitrogen. Tissues for determination of viral titers were processed to generate cell suspensions, then the cells lysed by three successive freeze-thaw cycles. BAL cells were likewise subjected to three successive freeze-thaw cycles. Virus titers were determined by plaque forming assays on BS-C-1 cells. Specifically, $250 \mu 1$ of each of the lysed cell suspensions and 10-fold serial dilutions of each were transferred directly onto BS-C-1 monolayers in multiwell tissue culture dishes, incubated for $48 \mathrm{hr}$, fixed and stained with crystal violet then plaques counted. Titers were calculated as p.f.u. /g tissue and p.f.u./mouse for BAL. The lower limit of detection is 120 plaques/g tissue and 180 plaques/mouse for BAL.

\subsubsection{Leukocyte isolation and Flow cytometry}

BAL fluid was collected from mock- and VACV-infected mice at the indicated time points p.i., as described (545). Briefly, $\mathrm{CCR} 5^{+/+}$and $\mathrm{CCR}^{-/-}$mice were sacrificed and the lungs were flushed with $3 \mathrm{ml}$ of PBS containing $10 \mathrm{U} / \mathrm{ml}$ heparin through a blunted 23gauge needle inserted into the trachea. Erythrocytes (RBC) in the pellets were lysed with $5 \mathrm{ml}$ of ACK buffer $\left(0.829 \% \mathrm{NH}_{4} \mathrm{Cl}, 0.1 \% \mathrm{KHCO}_{3}\right.$, and $0.0372 \% \mathrm{Na}_{2} \mathrm{EDTA}$, pH 7.4) for 5 minutes, followed by 2 washes with PBS. Spleen and mLN from each mouse were excised and digested for $30 \mathrm{~min}$ in digestion buffer (5\% calf serum, $1 \mathrm{mg} / \mathrm{ml}$ of 
collagenase (Roche diagnostics) and $30 \mu \mathrm{g} / \mathrm{ml}$ of DNase (Roche diagnostics in PBS). The digested tissue fragments were further dispersed by mashing and passing through a 0.7 micron cell strainer. Erythrocytes (RBC) in the pellets were lysed with $5 \mathrm{ml}$ of ACK buffer for 5 min followed by a 2 times wash with PBS to obtain a single cell suspension. Cells were stained for 45 minutes on ice with appropriate combinations of phycoerythrin (PE)-, phycoerythrin-Cy5 (PE-Cy5)- or allophycocyanin (APC)- labeled antibodies to CCR5 (CloneHM-CCR5, eBioscience), CD4 (clone GK1.5, eBioscience), CD8 (clone 53-6.7, eBioscience), CD3 (clone145-2C11, BD Pharmingen), CD11c (cloneHL3, BD Pharmingen), F4/80 (cloneBM8, eBioscience) and CD45 (clone 30-F11, eBioscience). The relevant isotype antibody controls were obtained from eBioscience and BD Pharmingen. After three washes with PBS, cells were fixed with $2 \%$ paraformaldehyde in PBS, washed and then analyzed by FACS (FACSAria). VACV-infected cells were

detected based on the EGFP expression from the tagged virus. Data were analyzed using FACSDiva software (BD Biosciences) and presented as the absolute number of positive cells within the gated population.

\subsubsection{Histology and Immunohistochemistry}

(a) Sample preparation. Tissues that were embedded in HistoPrep and snap frozen in liquid nitrogen were sectioned at $-20^{\circ} \mathrm{C}$ and then processed for immunohistochemistry. Specifically, thin sections were thawed at room temperature for 3 minutes followed by fixation in methanol/acetone (50:50) at $-20^{\circ} \mathrm{C}$ for 15 minutes, then washed with PBS before immunostaining. For paraffin sections, samples were fixed overnight at room 
temperature in $2 \%$ paraformaldehyde, dehydrated through a series of ethanol washes and processed for paraffin sectioning.

(b) Histological observations. Paraffin sections were rehydrated and stained with hematoxylin/eosin followed by standard histological protocols. Images were acquired with the Zeiss Axioplan 2 imaging microscope (Carl Zeiss, Canada Ltd) equipped with brightfield capabilities and a digital AxioCam camera (Carl Zeiss, Canada Ltd). Axio Vision 2.05 was used as the image acquisition software and captured images were processed with Adobe Photoshop version 7.0 (Adobe Systems, Inc.).

(c) Immunohistochemical analysis. Non-specific antibody binding was blocked by incubating thin sections in blocking buffer $(\mathrm{BSA}(0.5 \% \mathrm{wt} . / \mathrm{vol})+$ fish gelatin $(0.2 \%$ $\mathrm{vol} / \mathrm{vol}$ ) in PBS) for 30 minutes at room temperature, followed by incubation in wash buffer (BSA $(0.5 \% \mathrm{wt} . / \mathrm{vol})+$ fish gelatin $(0.1 \% \mathrm{vol} / \mathrm{vol})$ in PBS). PE or PE-Cy5 conjugated primary antibodies were appropriately diluted in blocking buffer, and thin sections incubated at room temperature for 1 hour. Antibodies against the following proteins were used: CCR5 (1:500), CD4 (1:250), CD8 (1:250). The relevant isotype antibodies were used as controls.

Sections were analyzed by confocal microscopy, as described previously (536).

\subsubsection{Bone marrow transplantation}

Bone marrow-derived cells from $\mathrm{CCR}^{+/+}$donor mice were obtained by flushing the cavity of freshly dissected femurs with PBS. The suspension was then centrifuged through Ficoll-Hypaque (Amersham Biosciences) for 30 minutes at room temperature at 
$1200 \mathrm{x}$ g. Hematopoietic leukocytes at the interphase were extracted and washed three times with PBS. Recipient $\mathrm{CCR} 5^{-/}$and $\mathrm{CCR} 5+/+$ control mice were irradiated with a sub-lethal dose of 1100 cGy by whole-body irradiation (WBI, 137Cs; Gammacell 40).

Four hours later mice received $10^{6}$ wild type $\left(\mathrm{CCR} 5^{+/}\right)$-derived hematopoietic leukocytes via tail vein injection. 2 weeks after bone marrow transplantation, mice received an intranasal inoculation of $10^{4}$ p.f.u. of VACV suspended in $20 \mu 1$ of sterile PBS. Mice were sacrificed at pre-determined time points, and BAL, lungs, $\mathrm{mLNs}$ and spleens were harvested for FACS analysis and VACV titration.

\subsubsection{Administration of TAK-779, a CCR5 antagonist}

A CCR5 antagonist, TAK-779 ( $N, N$-dimethyl- $N$-[4-[[[2- (4-methylphenyl)-6,7-dihydro$5 H$-benzocyclohepten-8-yl]carbonyl]amino]benzyl]tetra-hydro-2 $H$-pyran-4-aminium chloride; molecular weight $=531.13$ ) was kindly provided by Dr. Donald Branch (University of Toronto). TAK-779 was dissolved in 5\% manitol solution and mice were injected subcutaneously with $150 \mu \mathrm{g}$ TAK-779 in a volume of $100 \mu 1,1$ day prior to and daily p.i.

\subsubsection{Statistical analysis}

Statistically significant differences in measured outcomes (other than virus titer) between groups of mice were determined by Student's $t$ test using Microsoft Excel MAC v.X software. p-values $<0.05$ were considered significant.

Differential Analysis of tissue virus titers: For each tissue type, we first fit a two-way analysis of variance (ANOVA), to evaluate the significance of all the data points in one set compared to all the data points in the comparator set (with two levels: $\mathrm{CCR} 5^{+/+}$vs 
$\left.\mathrm{CCR}^{-/-}\right)$. We then performed a Tukey honest significance difference test to compare the means of the levels of each data point conditional on the time point, using the ANOVA results. 
3.7. Supplementary Figures 


\section{Supplementary Figure 3.1}

Intranasal infection with VACV WR or EGFP- VACV induces similar immune responses

Groups of female CCR5 ${ }^{+/+}$mice $(n=5)$, age $6-8$ wks, were infected with $10^{4}$ p.f.u. of either VACV strain WR ( $\square$ ) or EGFP- VACV $(\square)$ by intranasal inoculation. Immune cell infiltrates were measured in the BAL, spleens and mLNs of all mice. Values are mean cell counts $\pm \mathrm{SE}$ and are representative of two independent experiments. 
Supplementary Figure 1
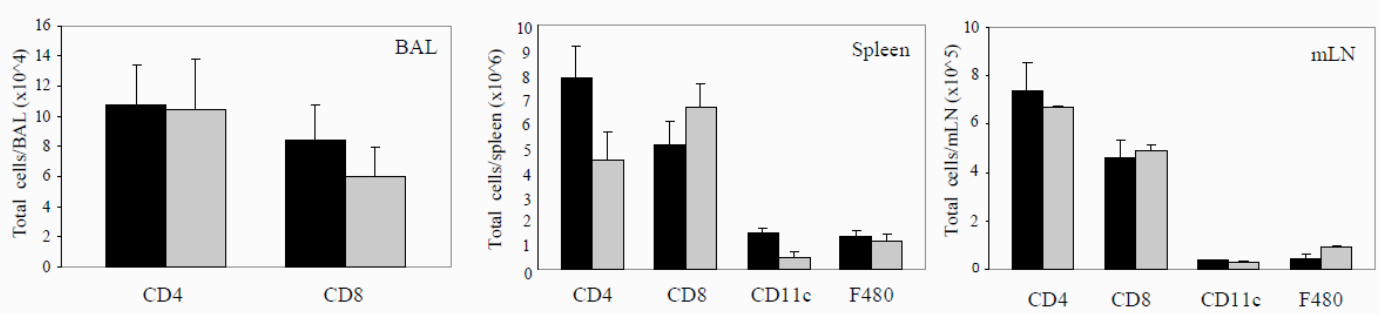


\section{Supplementary Figure 3.2}

Intranasal inoculation with VACV leads to an influx of CD4+ and CD8+ $\mathrm{T}$ cells into the lungs of $\mathrm{CCR5}^{+/+}$but not $\mathrm{CCR5}^{-/-}$mice

$\mathrm{CCR}^{+/+}$and $\mathrm{CCR}^{-/-}$mice were infected by intranasal inoculation with $10^{4}$ p.f.u. of EGFP-VACV and lung tissues were processed as frozen sections as described in Materials and Methods. Sections were stained with Cy3-conjugated anti- CCR5 antibody and PE-Cy5 conjugated anti- CD4/CD8 antibodies and analyzed by confocal microscopy. Distribution of CD4+ T cells in the lungs of mock or A5-EGFP-VACV infected (A, B) $\mathrm{CCR}^{+/+}$and $(\mathrm{E}, \mathrm{F}) \mathrm{CCR}^{-/-}$mice. Distribution of CD8+ T cells in the lungs of mock- or A5-EGFP-VACV- infected $(\mathrm{C}, \mathrm{D}) \mathrm{CCR}^{+/+}$or $(\mathrm{G}, \mathrm{H}) \mathrm{CCR}^{-/-}$mice. The data are representative of two independent experiments. 
Supplementary Figure 2

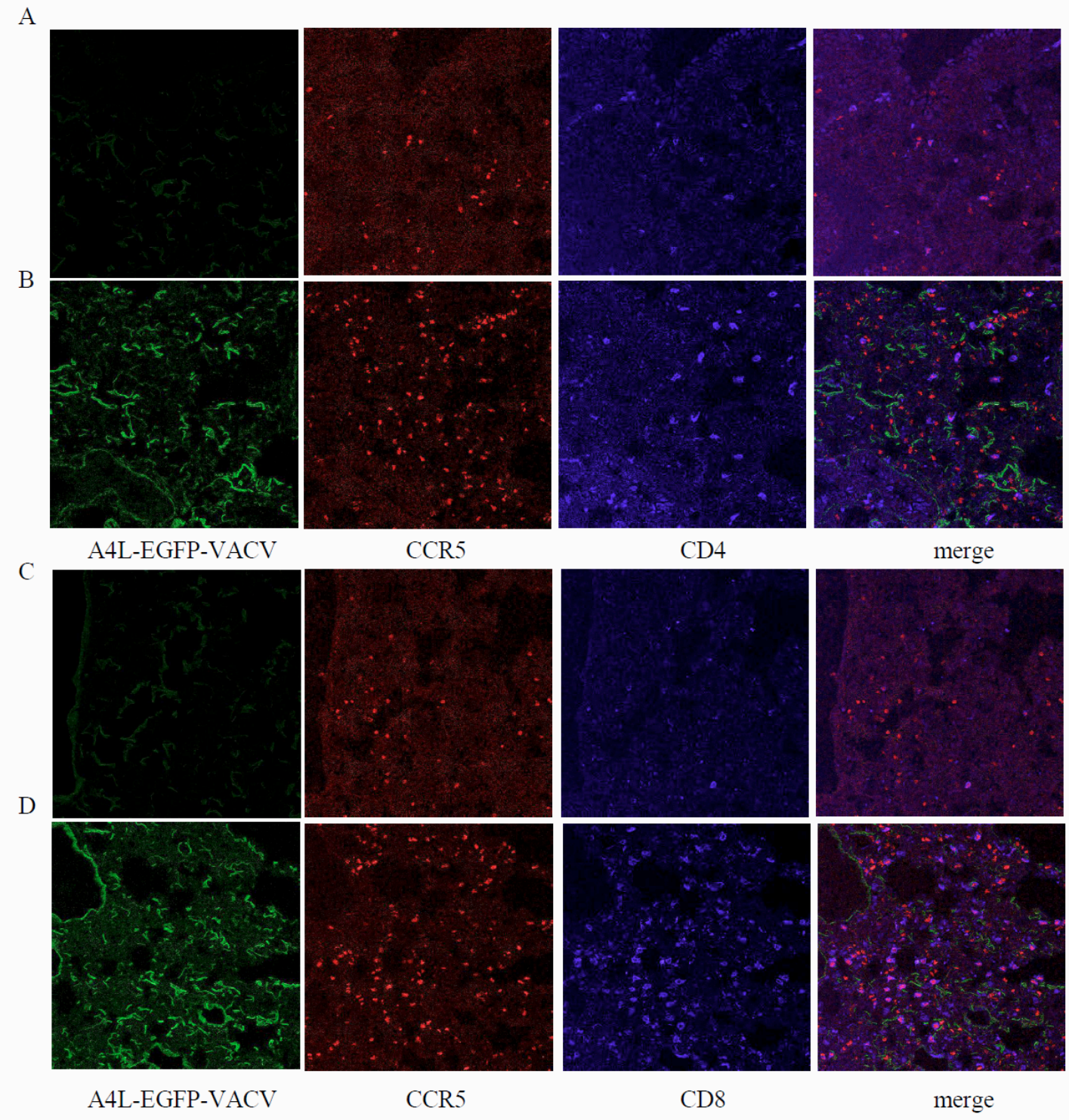


Supplementary Figure 2 continued

E
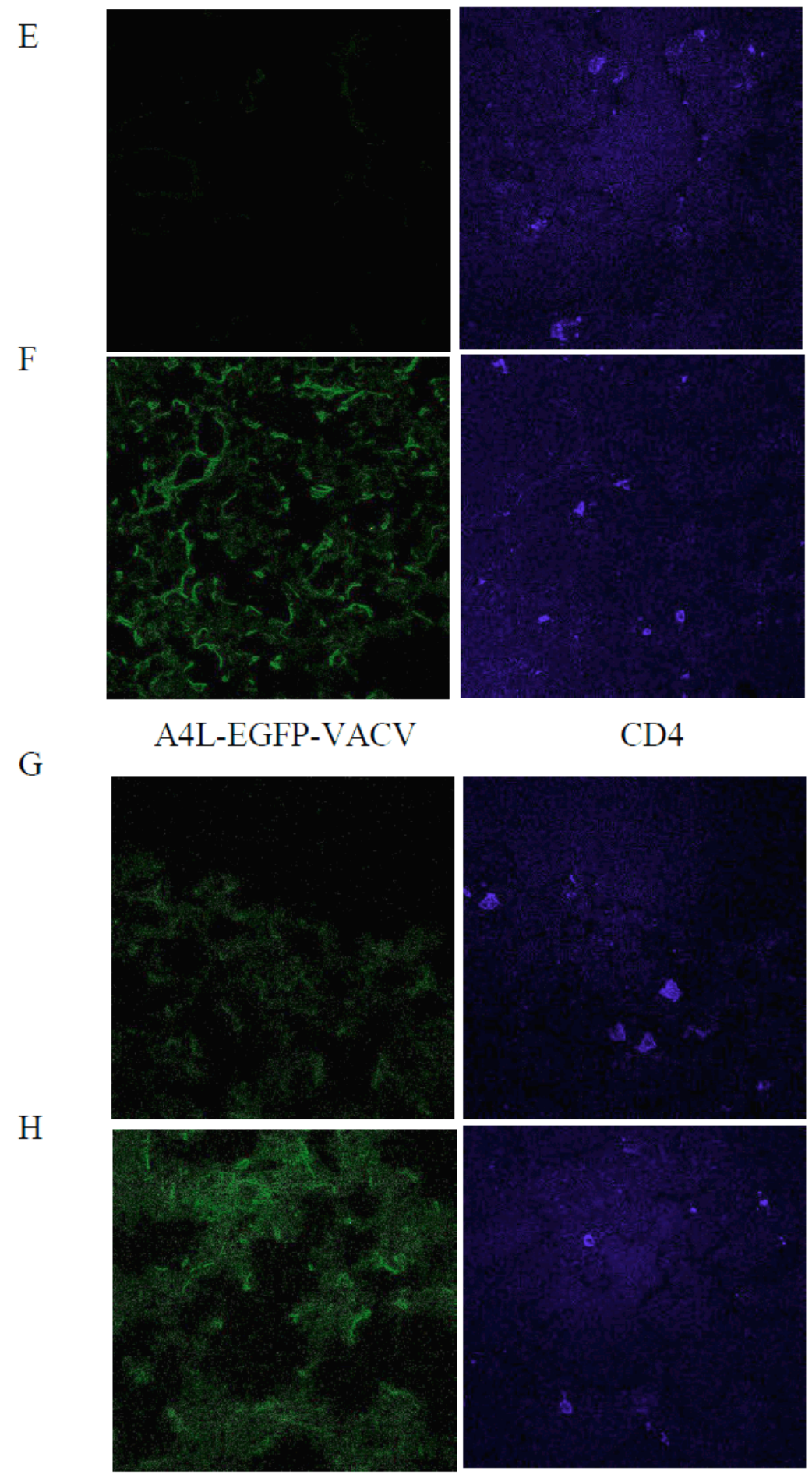

CD4

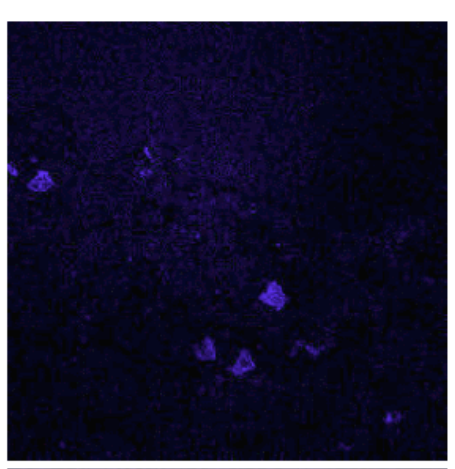

A4L-EGFP-VACV

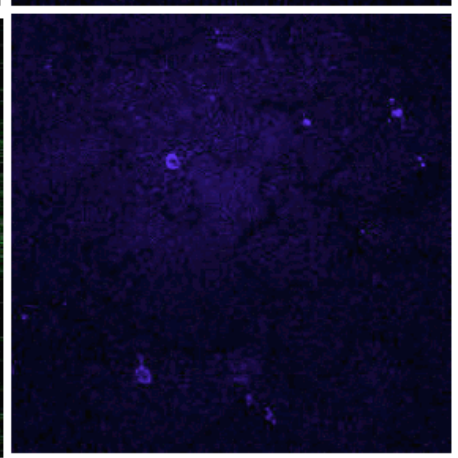

CD8 


\section{Supplementary Figure 3.3}

Intranasal infection with VACV results in activation of CD4+ and CD8+ $T$ cells in mLN of $\mathrm{CCR}^{+/+}$but not $\mathrm{CCR5}^{-/-}$mice

Groups of female CCR $5^{+/+}$mice $(n=5), 6-8$ wks, were infected with $10^{4}$ p.f.u. of VACV by intranasal inoculation. CD4+ and CD8 $+\mathrm{T}$ cell activation was determined in the $\mathrm{mLN}$ using FITC-conjugated anti-CD621 (A), PE-conjugated anti CD69 (B), PE-Cy5conjugated anti- CD4, CD8 and APC-conjugated CD3 antibodies.

(口) $\mathrm{CCR}^{+/+} ;(\square), \mathrm{CCR} 5^{-/-}$ 
Supplementary Figure 3

A
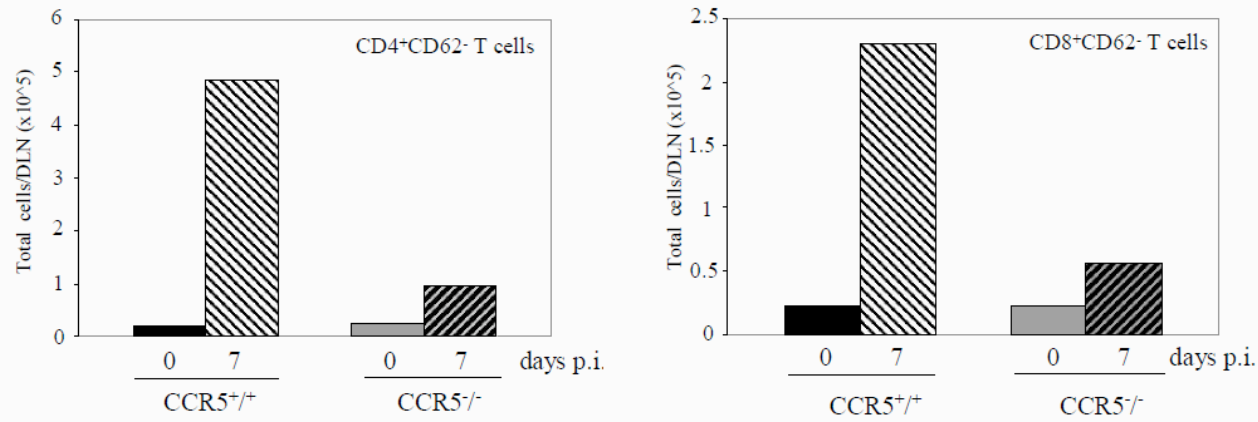

B
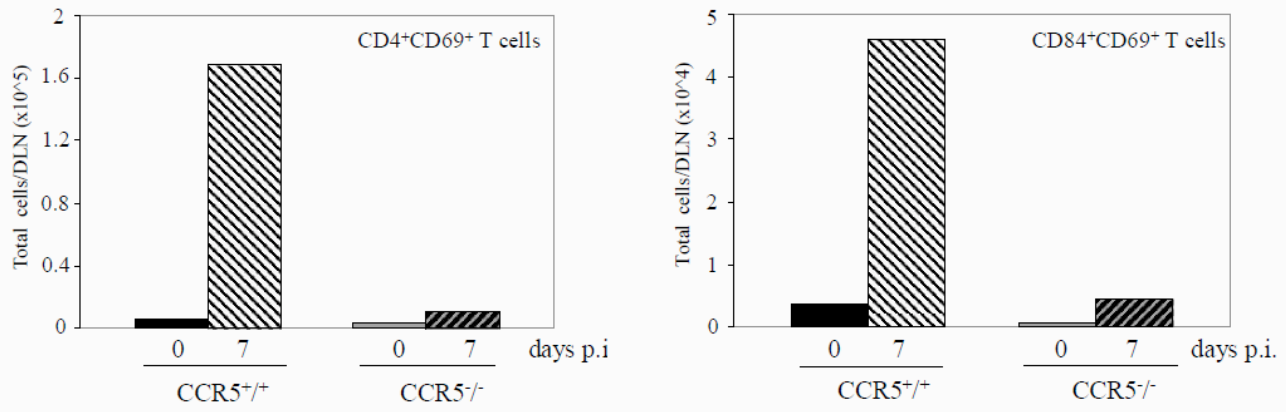


\section{Supplementary Figure 3.4}

Intranasal infection with $10^{5}$ p.f.u. of VACV results in immunological response in $\mathrm{CCR5}^{+/+}$and to a lesser extent in $\mathrm{CCR5}^{-/-}$mice

Groups of female mice $(n=10)$ age $6-8$ wks were infected with $10^{5}$ p.f.u. of VACV by intranasal inoculation. $\mathrm{CD}^{+} \mathrm{CD}^{+}$and $\mathrm{CD}^{+} \mathrm{CD}^{+}, \mathrm{CD} 11 \mathrm{c}^{+}$and $\mathrm{F} 4 / 80^{+}$cells present in BAL (A), spleens (B) and mLNs (C) of VACV infected $\mathrm{CCR}^{+/+}$and $\mathrm{CCR} 5^{-/-}$mice were quantitated by flow cytometry on days 7 p.i.. Cells were stained with PE-Cy5-conjugated anti- CD4, CD8, F4/80 and APC-conjugated CD11c and CD3 antibodies.

( ) $\mathrm{CCR}^{+/+} ;(\square), \mathrm{CCR}^{-/-}$. Data are presented as mean cell counts $\pm \mathrm{SE}$ and are representative of two independent experiments. 
Supplementary Figure 4
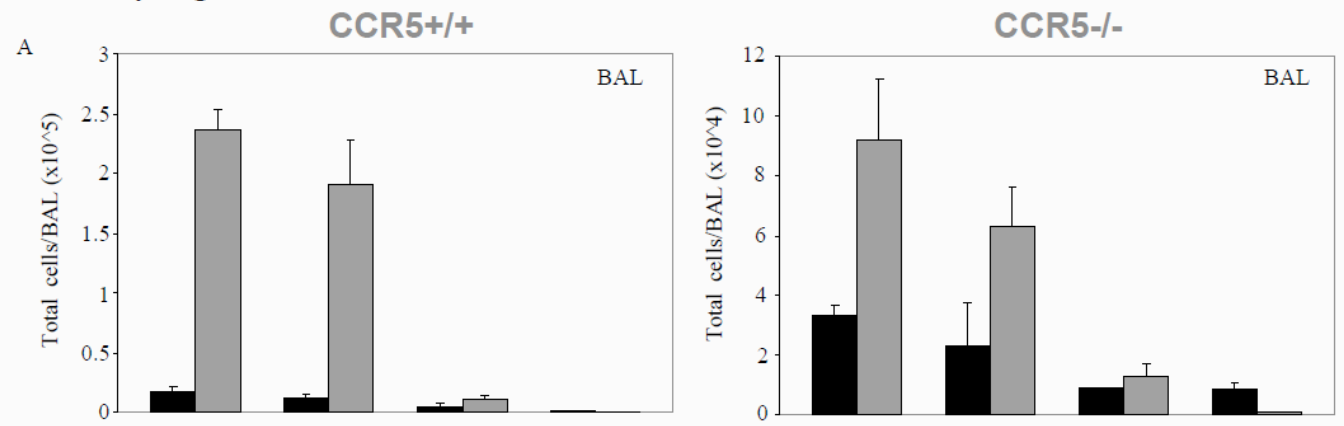

B
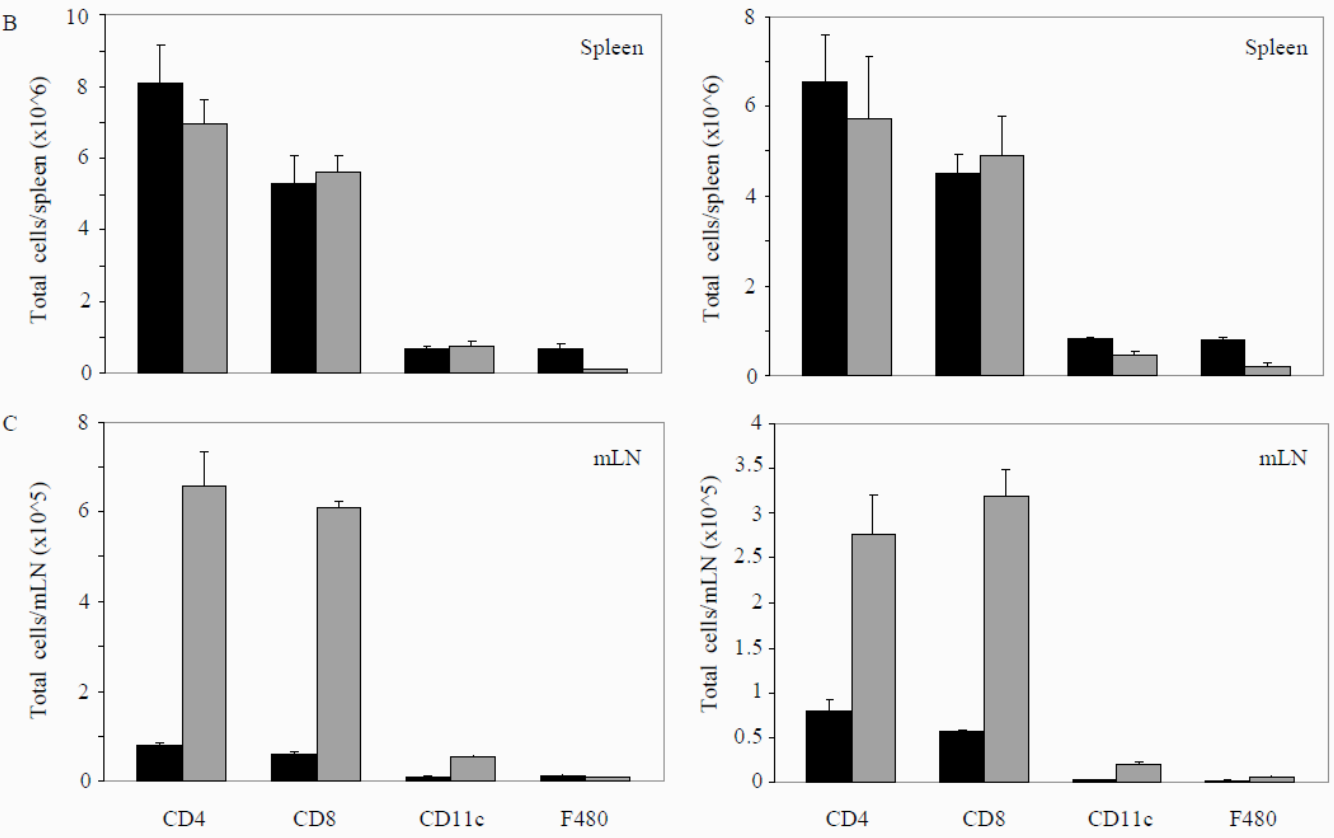


\section{Supplementary Figure 3.5}

\section{$\mathrm{CCR5}^{-/-}$mice are permissive for influenza A/WSN/33 infection}

A) Group of female mice ( $n=5)$ aged 6-8 wks, were infected with 1 H.A.U. influenza A/WSN/33 virus and body weight measured daily. Body weight loss was expressed as the mean percent weight loss at the indicated time points compared to that of controls $\pm \mathrm{SE}$.

$\left(\rightarrow \mathrm{CCR}^{+/+} ;(\square), \mathrm{CCR}^{-/-}\right.$(B) Lungs of mock- and influenza A/WSN/33- infected $\mathrm{CCR}^{+/+}$and $\mathrm{CCR}^{-/-}$mice were harvested on days 3 and 6 p.i., fixed in $2 \%$ paraformaldehyde and embedded in paraffin. $6 \mu \mathrm{m}$ thick histological sections were prepared and stained with hematoxylin and eosin. $\mathrm{CD} 3+\mathrm{CD} 4+$ and $\mathrm{CD} 3+\mathrm{CD} 8+, \mathrm{CD} 11 \mathrm{c}+$ and F4/80+ cells present in BAL, spleens and mLNs of influenza A/WSN/33-infected $\mathrm{CCR}^{+/+}$and $\mathrm{CCR}^{-/}$mice were determined on days 3 (C) and 6 (D) p.i. Cells were stained with PE-Cy5-conjugated anti- CD4, CD8, F4/80 and APC-conjugated CD11c, CD3 antibodies.

(a ) $\mathrm{CCR}^{+/+} ;(\square), \mathrm{CCR}^{-/-}$. Data are presented as mean cell counts $\pm \mathrm{SE}$ and are representative of two independent experiments. 
Supplementary Figure 5

A

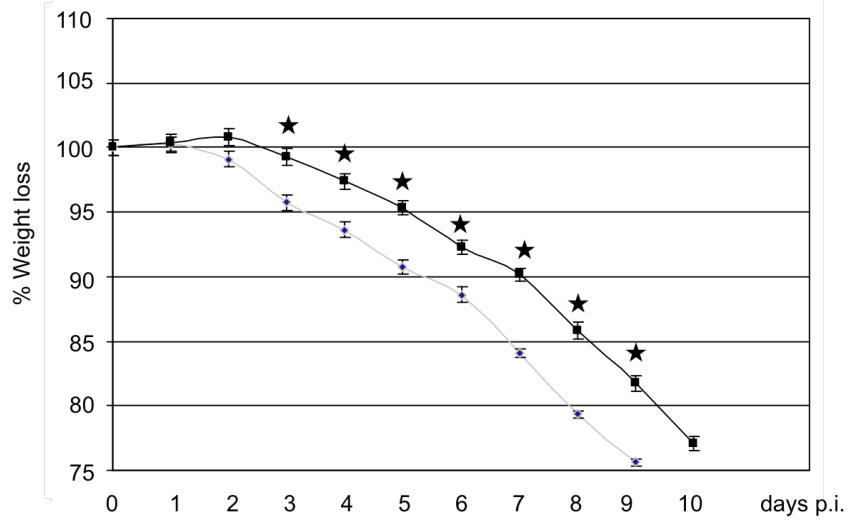

B
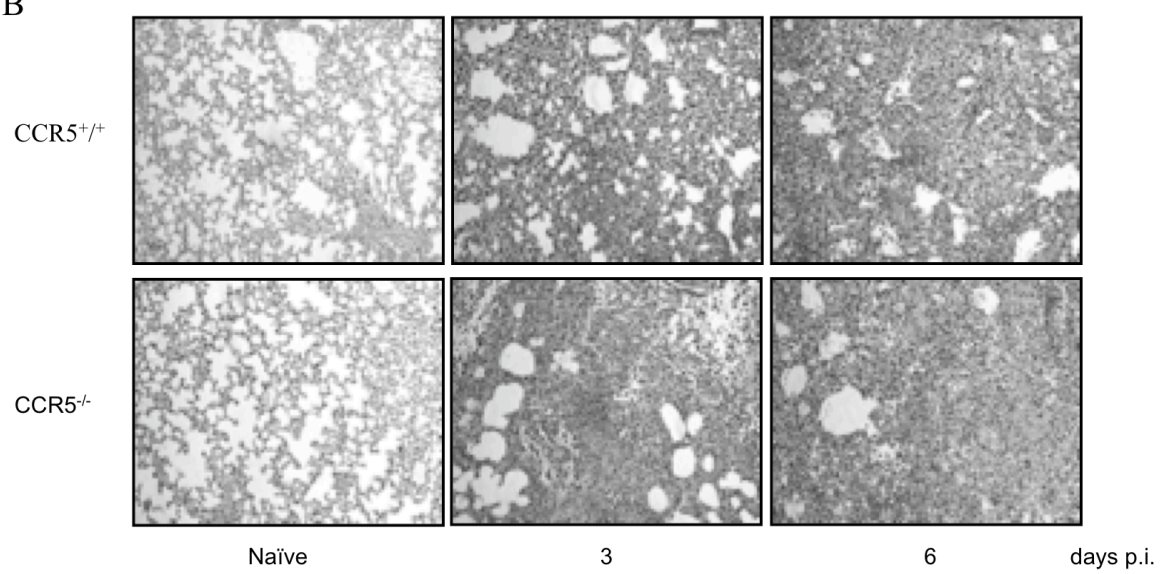

C
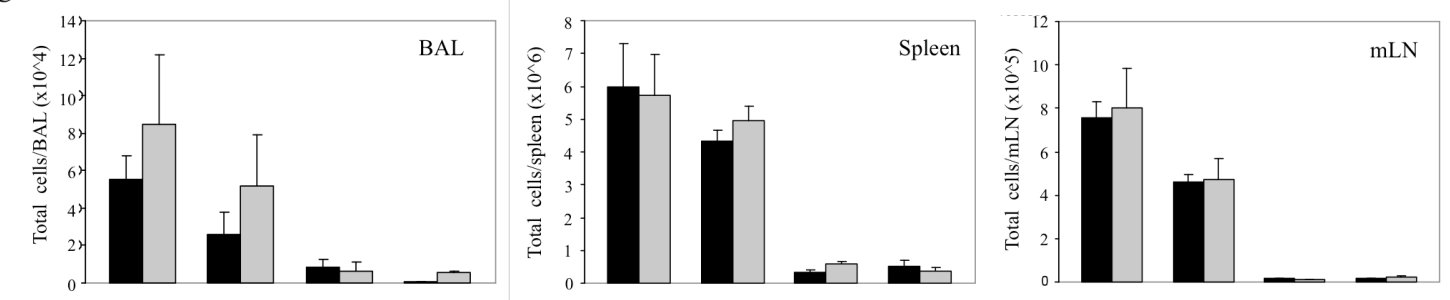

D
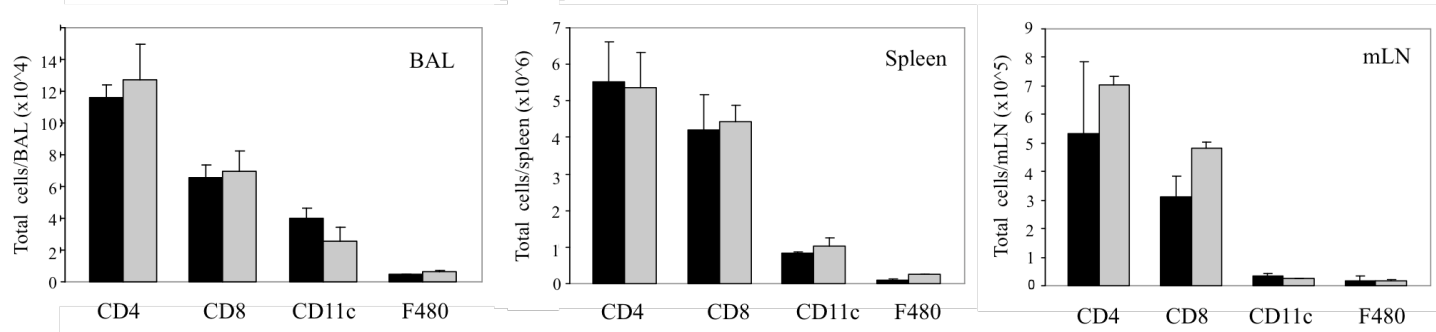


\section{Supplementary Figure 3.6}

\section{$\mathrm{CCR5}^{-/-}$mice are permissive for influenza $\mathrm{A} / \mathrm{WSN} / 33$ infection}

A) Groups of female mice ( $n=5), 6-8 w k s$, were infected with 5 H.A.U. influenza A/WSN/33 virus and their body weight measured daily. Body weight loss was expressed as the percent weight loss of animals at the indicated time points compared to that of

controls \pm SEM. ( ) $\mathrm{CCR}^{+/+} ;(\square), \mathrm{CCR}^{-/-}$(B) Lungs of mock- and influenza $\mathrm{A} / \mathrm{WSN} / 33$ infected $\mathrm{CCR}^{+/+}$and $\mathrm{CCR}^{-/-}$mice at day 5 p.i. were fixed in $2 \%$ paraformaldehyde and embedded in paraffin. $6 \mu \mathrm{m}$ thick histological sections were prepared and stained with hematoxylin and eosin. CD4+, CD8+ T cell, CD11c+ and F4/80+ infiltration into BAL (C), spleens (D) and mLNs (E) of influenza A/WSN/33 infected $\mathrm{CCR}^{+/+}$and $\mathrm{CCR} 5^{-/}$mice were determined. Cells were stained with PE-Cy5conjugated anti- CD4,CD8, F4/80 and APC-conjugated CD11c, CD3 antibodies.

$\left.\mathrm{CCR}^{+/+} ;{ }^{\square}\right), \mathrm{CCR}^{-/-}$. Data are presented as mean cell counts $\pm \mathrm{SE}$ and are representative of two independent experiments. 


\section{Supplementary Figure 7}

A

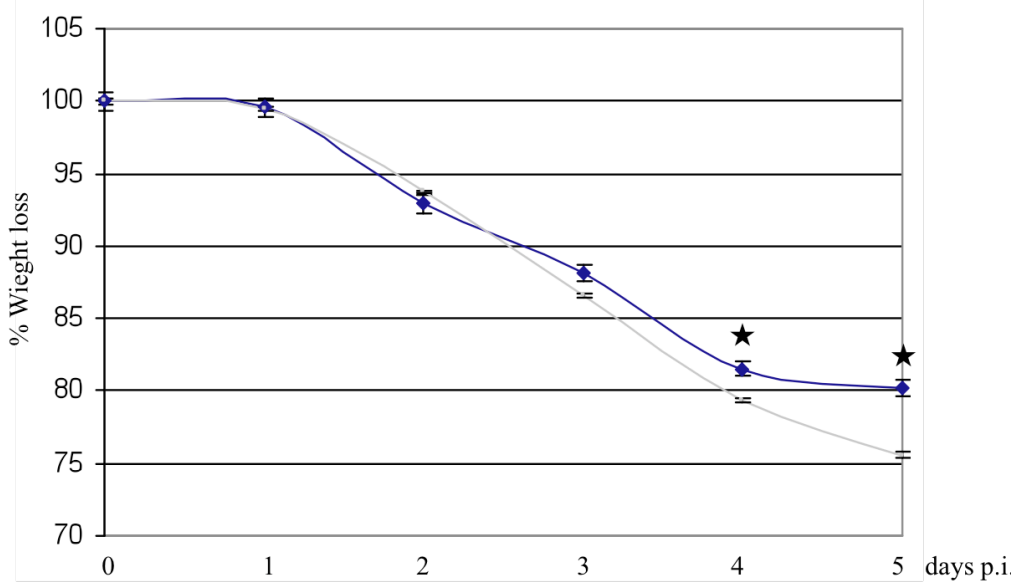

B

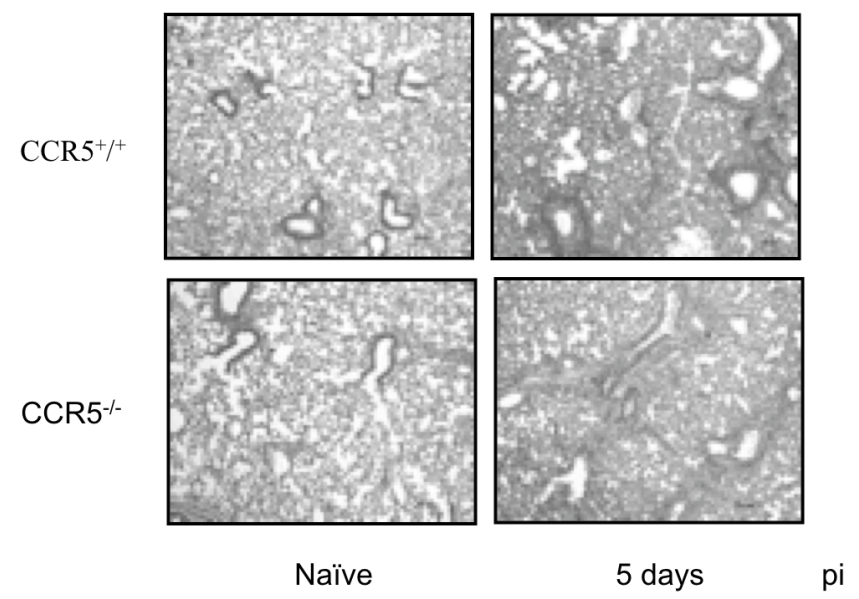

C
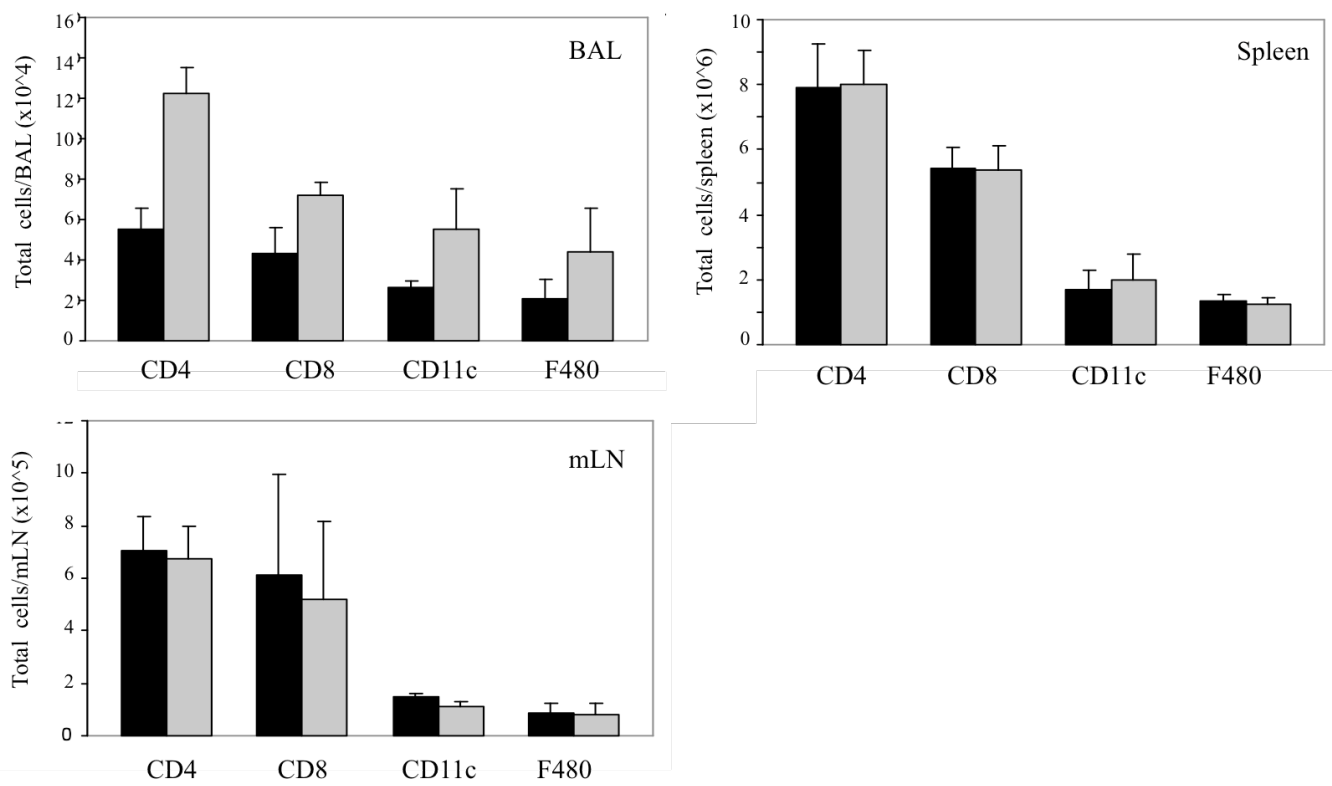


\section{Chapter 4}

\section{Glomulin, a protein involved in VACV infection}

\section{Chapter 4 is a manuscript submitted as:}

Rahbar R., Murooka T., Kislinger T. and Fish E.N. (2009). Glomulin, a protein involved in VACV infection

R.R. performed all experiments (except for mass spectrometry), analyzed the data and drafted the manuscript. 


\subsection{Abstract}

Using mass spectrometry-based proteomics (i.e. MudPIT), glomulin was identified as a host cell protein that interacts with vaccinia virus (VACV). Knockdown of glomulin expression in human PM1.CCR5 T cells reduces VACV infection. Glomulin interacts with the inactive, non-phosphorylated form of c-MET. Upon c-MET activation, glomulin is released in a phosphorylated form, leading to the subsequent phosphorylation of p 70 S6kinase (p70S6K). We demonstrate that treatment of PM1.CCR5 T cells with a c-Met phosphorylation inhibitor leads to a significant reduction in VACV infectivity. The data indicate that inhibition of c-Met phosphorylation, reduces the cytosolic availability of activated glomulin, thus leading to a decrease in VACV infectivity. These data identify glomulin as a permissivity factor for VACV infection, and as a potential therapeutic target for $\mathrm{VACV}$.

\subsection{Introduction}

Vaccinia virus (VACV) is the best-known prototypic member of the Poxviridae family. Members of the poxvirus family are characterized by a complex enveloped brickshaped virion, a large genome that ranges from 130 to $300 \mathrm{~kb}$ (451). VACV has a large double-stranded DNA genome of approximately $200 \mathrm{~kb}$ and undergoes a complicated replicative cycle within the cytoplasm of infected cells. The control of gene expression occurs in three phases, each step being strictly dependent on the success of the previous phase (454). Although poxviruses possess well-organized replication machinery that allow them to infect a wide spectrum of eukaryotic hosts in vitro, some poxviruses are host restricted $(173,174,192,215,286,288,421,670,763)$. In these restrictive cells, 
poxviruses efficiently enter target cells, but fail to complete their replicative life cycle. This is due to their inability to circumvent innate intracellular barriers necessary for viral replication, or the absence of necessary complementing factors provided by the host cell $(423,536)$. Consequently, the ability of poxviruses to manipulate signal transduction within the host cell has a major impact on infectivity, as cell tropism is dependent on the virus-inducible expression of host derived genes $(315,423,742)$. In an earlier publication we provided evidence for the intracellular peripheral colocalization of VACV and CCR5 in permissive cells (536). In nonpermissive cells, the apparent block in viral replication we observed coincided with a block in late gene expression. Our working hypothesis is that VACV colocalization with CCR5 results in CCR5 activation, mediated either by direct contact between the viral core proteins and CCR5, or by viral proteins interacting with cellular factors that then activate CCR5. CCR5 activation leads to the phosphorylation-activation of distinct signaling effectors that prescribe alterations in cell gene expression to promote a favourable environment for VACV late gene expression (536). Notably, there is also evidence that a cellular factor is required for transcription of VACV intermediate-stage genes. (569).

Herein, we used MudPIT on a LTQ linear ion-trap, to describe a novel function for glomulin, namely as a permissivity factor for VACV infection of PM1.CCR5 human $\mathrm{T}$ cells. We also provide evidence for the involvement of the c-Met receptor in VACV infection. 


\subsection{Results}

\subsubsection{A4L-EGFP-VACV interacts with multiple host proteins}

In an earlier report we provided evidence for co-localization of the VACV core with the chemokine receptor CCR5 (536). This co-localization precedes virus-inducible signaling and virus replication. To identify specific cell proteins that interact with VACV and, therefore, may be host-derived permissivity factors for infection, we undertook a mass spectrometry-based screen for potential VASCV-binding proteins. Permissive PM1.CCR5 and non-permissive PM1.CCR5.Y339F human T cells were either left untreated or infected with A4L.EGFP.VACV at an m.o.i. of 10. One-hour post infection (p.i.) cells were lysed and protein samples prepared for mass spectrometry, as described in Material and Methods. We employed a multidimensional protein identification technology (MudPIT), that incorporates an HPLC interface with linear ion trap mass spectrometry, and identified 387 unique proteins that associated with A4L-EGFP-VACV in infected PM1.CCR5 cells, 215 proteins in PM1.CCR5Y339F cells and 127 proteins in uninfected PM1.CCR5 T cells (Figure 4.1A). To better distinguish correct from incorrect peptide identifications, we performed a target/decoy database strategy to empirically determine the false discovery rate (FDR). Based on these rigorous criteria we estimate our FDR to $\sim 0.5 \%$ ( 8 decoy hits in the final dataset). Table 4.1 shows the number of decoy proteins passing the filter. Figure 4.1B identifies the biological processes that these 387 proteins are involved in, and their molecular functions are indicated in Figure 4.1.C. Specific gene identifiers as well as the number of proteins in each group are shown in Supplementary Tables 4.1 and 4.2. 


\subsubsection{Knockdown of glomulin reduces VACV infection}

Among the 387 proteins revealed in the PM1.CCR5-unique cluster, glomulin attracted our attention based on its ability to associate with a transmembrane tyrosine kinase receptor, to become phosphorylated and to influence downstream signaling associated with protein translation (116). In earlier studies, we and others have provided evidence for the importance of tyrosine phosphoryation events in poxvirus infectivity $(416,536)$. To investigate the contribution of glomulin to VACV infectivity, expression of glomulin was blocked by transfection of PM1.CCR5 T cells with glomulin-specific siRNA duplexes. Introduction of glomulin-specific siRNA duplexes, but not scrambled siRNA, in PM1.CCR5 cells resulted in significant down-regulation of glomulin mRNA at $48 \mathrm{~h}$ p.i. (Figure 4.2A). We have previously shown that cell surface CCR5 expression is critical for VACV infection of PM1 T cells (536). To investigate whether reduction in VACV infection is due to diminished ectopic expression of CCR5 post transfection with the glomulin-specific siRNA, cell surface CCR5 expression was determined by flow cytometric analysis using anti-CCR5-specific antibodies. As shown in Figure 4.2B, cell surface expression of CCR5 is unaffected by siRNA transfection. Notably, cells exhibitng reduced glomulin expression exhibited a reduction in the level of viral infection (Fig $4.2 \mathrm{C})$. 


\title{
Figure 4.1
}

\section{MudPIT identifies host proteins that physically associate with A4L-EGFP-VACV}

\begin{abstract}
A)Venn diagram depicting host cell proteins that associate with VACV in permissive PM1.CCR5 and non-permissive PM1.CCR5.Y339F infected with 10 m.o.i. of A4LEGFP-VACV. 387 proteins bind exclusively to A4L-EGFP-VACV in PM1.CCR5 T cells. B) Biological processes associated with proteins identified in the PM1.CCR5 cluster. C) Molecular function of proteins in PM1.CCR5 cluster.
\end{abstract}


Figure 1

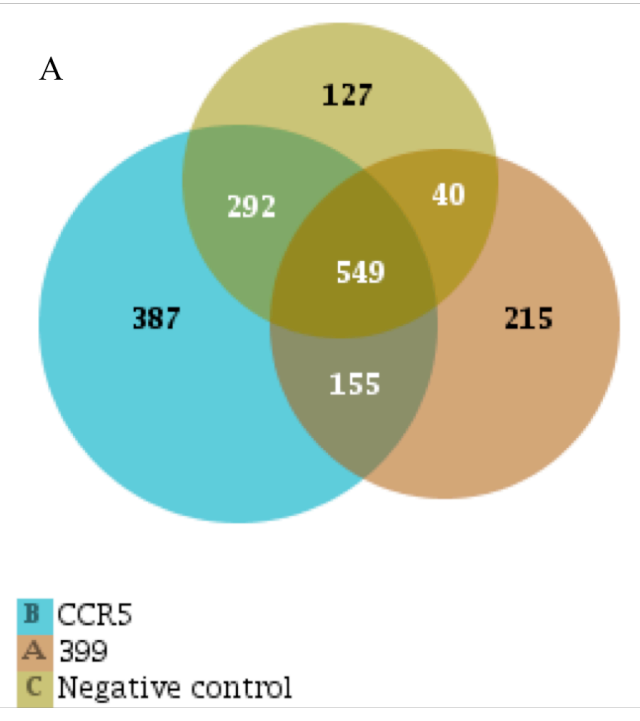

A 399

C Negative control

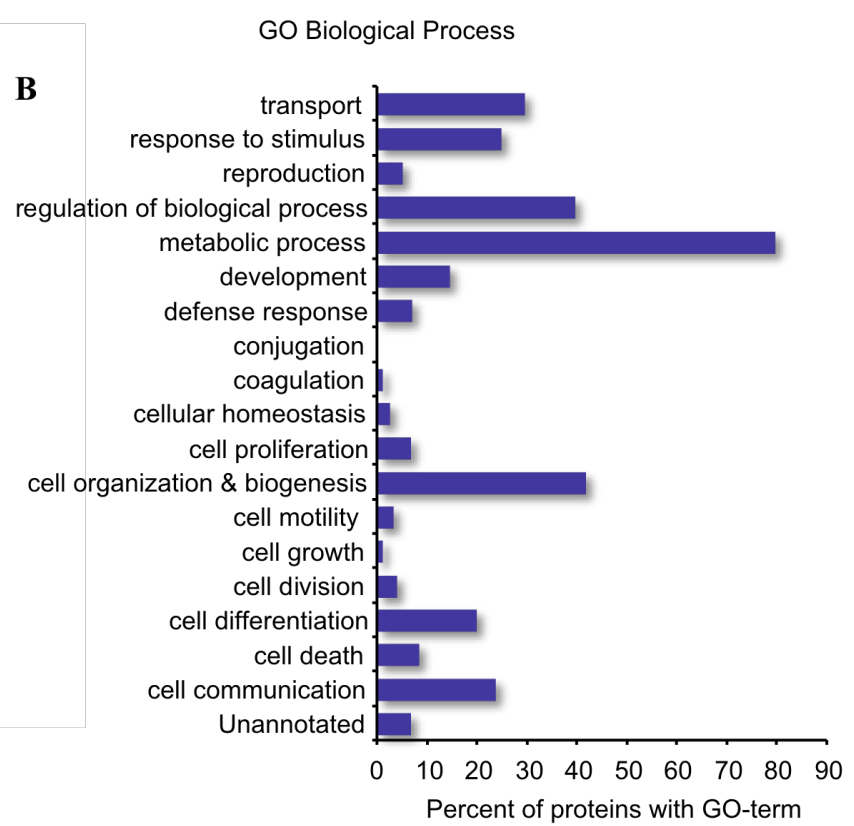

C

GO Molecular Function

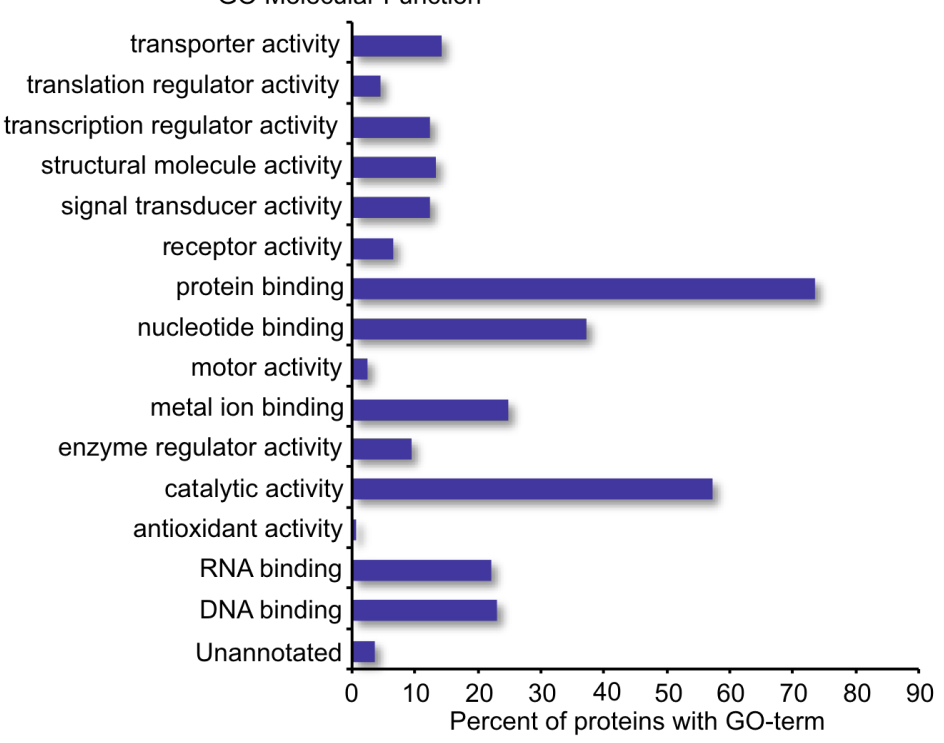




\section{Table 4.1}

Empirical evaluation of false recovery rate

\begin{tabular}{r|ccc} 
& Decoy proteins & Target proteins & Unique peptides \\
\hline CCR5Y339F & 5 & 980 & 5639 \\
CCR5 & 0 & 1399 & 7854 \\
Neg. control & 3 & 1024 & 5523 \\
\hline Total & 8 & 1837 & 12552
\end{tabular}




\section{Figure 4.2}

\section{Knockdown of glomulin reduces VACV infection}

(A) PM1.CCR5 T cells were transfected with either scrambled siRNA or with siRNA specific for glomulin. Cells were harvested for RNA extraction $48 \mathrm{~h}$ posttransfection. Reverse-transcription PCR for glomulin was performed. A probe specific for human ßactin was included to monitor transcription of a cellular mRNA. (B) PM1.CCR5 T cells were left untreated (gray solid line) or transfected with either glomulin-specific siRNA (dotted gray line) or the control, scrambled siRNA (dotted black line). At 48 h posttransfection, cell surface CCR5 expression was determined by flow cytometric analysis using anti-CCR5-specific antibodies. Filled (negative) cytograms correspond to IgG reagents alone. (C) PM1.CCR5 T cells were left untreated or transfected with either glomulin-specific siRNA or the control, scrambled siRNA. 48 hrs post-transfection., cells were infected with VACV at an m.o.i. of 1. 24 hrs p.i., the cells were analyzed the extent of virus infection using the $\beta$-galactosidase colorimetric assay described in Materials and

Methods. ( $\square$ ) PM1.CCR5 cells infected with VACV; ( $\boldsymbol{q}$ ) PM1.CCR5 cells transfected with the control, scrambled siRNA, and then VACV infected; ( $\mathbf{q})$, PM1.CCR5 cells transfected with glomulin-specific siRNA and then VACV infected. Values are means \pm standard errors and are representative of two independent experiments. 
Figure 2

A

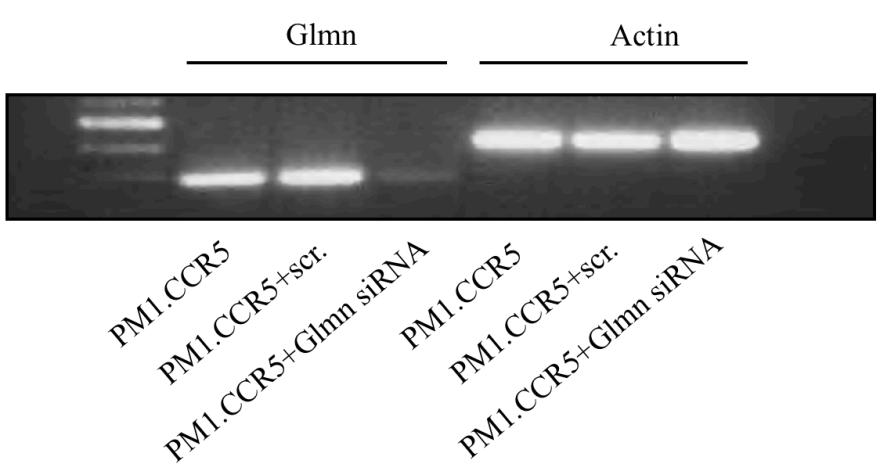

C

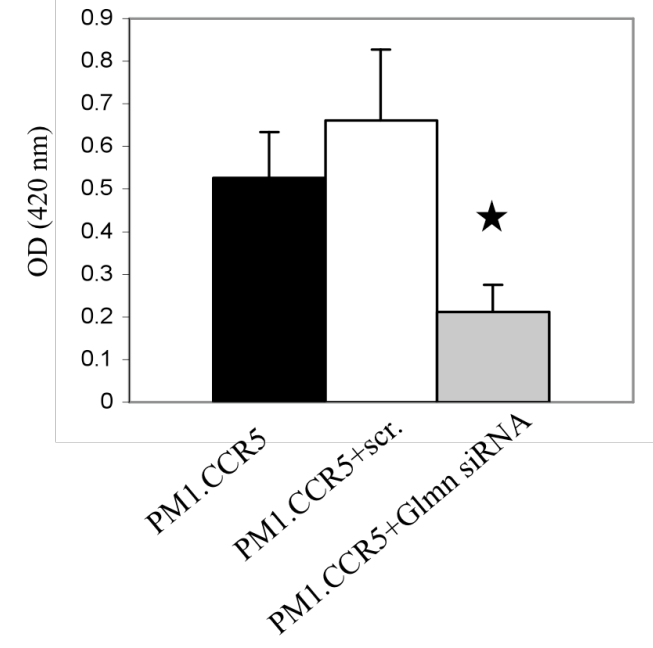

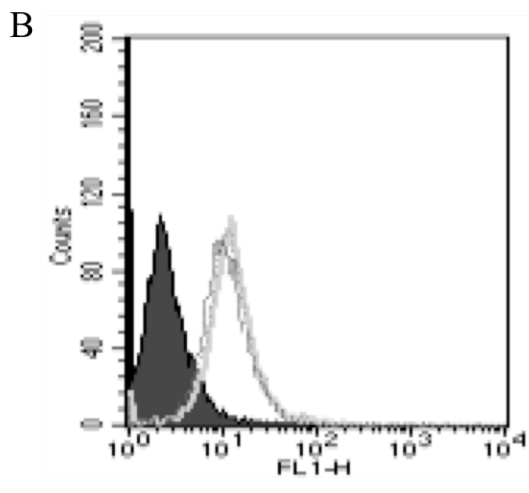




\subsubsection{Inhibition of cMET-mediated phosphorylation reduces VACV infection}

Previous studies using a yeast two-hybrid system have shown that glomulin interacts with the proto-oncogene HGF receptor, c-Met, specifically within the last 30 amino acids of the C-terminus (243). Glomulin interacts with the inactive, nonphosphorylated form of c-MET, but is released upon phosphorylation of c-MET. Accordingly, in the next series of experiments we investigated the contribution of c-Met to VACV infection. In dose-response cytotoxicity experiments we determined that $\leq 10^{8}$ $\mathrm{nM}$ of a c-Met inhibitor had no effect on PM1.CCR5 cell survival. Figure 4.3B shows the basal ectopic expression level of cMet in PM1.CCR5 cells. Data presented in Figure 4.3.C show VACV infection of PM1.CCR5 cells results in phosphorylation of c-Met at 30 min. p.i. and that 16 hours pre-treatment with $10^{7} \mathrm{nM}$ of the c-Met inhibitor resulted in no discernible VACV-induced c-MET phosphorylation above baseline levels (Figure 3C). In subsequent experiments we provide evidence that PM1.CCR5 cells treated with the c-Met inhibitor at $10^{8} \mathrm{nM}$ protected PM1.CCR5 cells from VACV infection (Figure 4.3D). 


\section{Figure 4.3}

\section{Inhibition of c-MET activation reduces $\mathrm{VACV}$ infection}

PM1.CCR5 T cells were left untreated or treated with either $10^{8} \mathrm{nM}$ DMSO or cMet inhibitor $1 \mathrm{hr}$ prior to infection, then infected with VACV at an m.o.i. of $1.24 \mathrm{hrs}$ p.i. (A) Cell surface CCR5 expression was determined by flow cytometric analysis using antiCCR5-specific antibodies. (B) cMet expression was determined by flow cytometric analysis using anti-cMet-specific antibody. (C) At 30 minutes post-infection, cMet phosphorylation was detected by flow cytometric analysis using anti-pcMet-specific antibody. Filled cytogram: IgG control alone, gray solid line: pcMet 30 minutes post infection, light dotted line: basal pcMet, heavy dotted line: pcMet 30 post infection in cells treated with cMet inhibitor. (D) The cells were also analyzed for $\beta$-galactosidase activity by means of a colorimetric assay (see Materials and Methods). ( $\quad$ ) PM1.CCR5 cells infected with VACV; ( $\quad$ ), PM1.CCR5 cells treated with the DMSO and then VACV infected; ( ), PM1.CCR5 cells treated with cMet inhibitor and then VACV infected. The means \pm standard errors representative of two independent experiments. T: treated with cMet inhibitor, $\mathrm{U}$ : untreated 
Figure 3

A

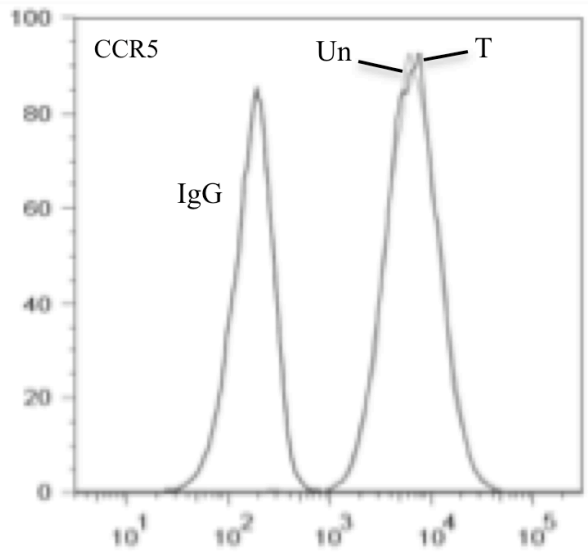

C

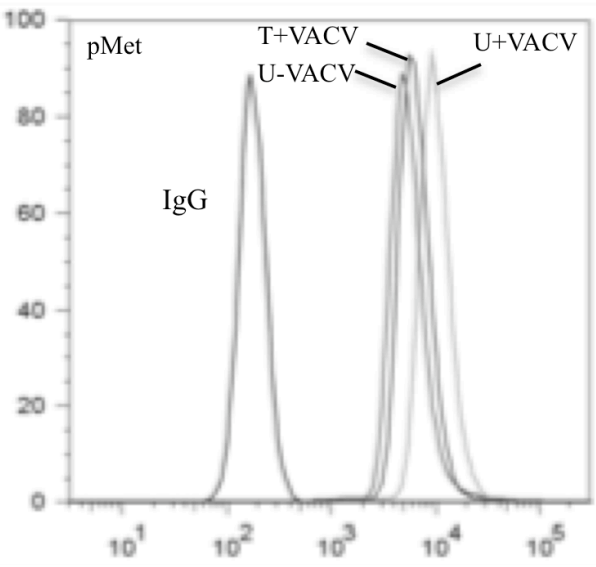

D

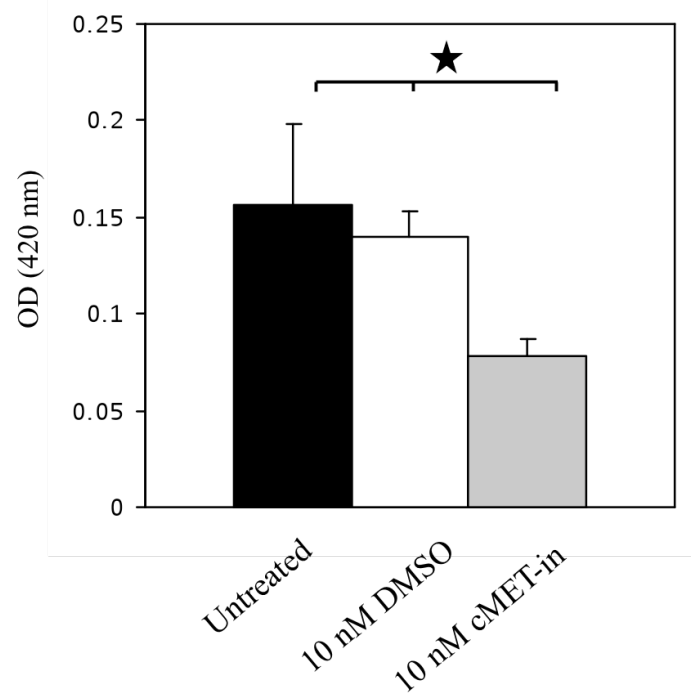

B
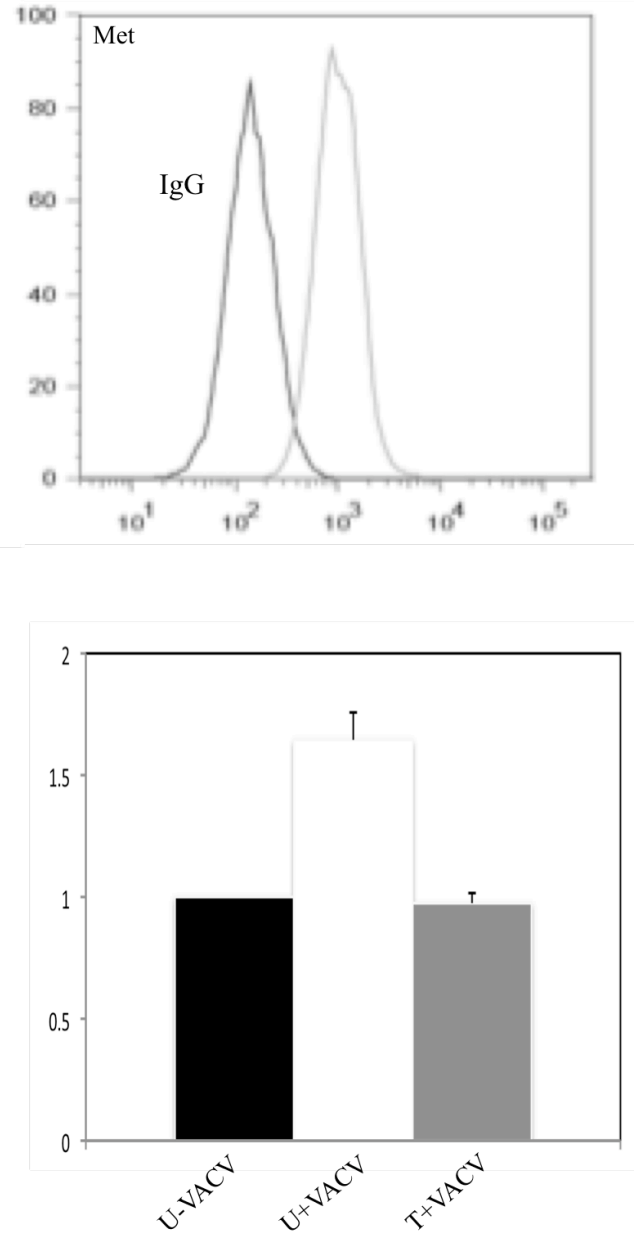


\subsection{Discussion}

VACV encodes a considerable number of proteins that counteract host defense mechanisms against virus infection. These host range and immunomodulatory proteins mimic host-derived molecules. Virokines are secreted proteins that mimic cytokines, complement regulators, or other humoral inhibitors. Viroceptors, which are secreted or localized to the surface of infected cells, are protein mimetics of cytokine/chemokine receptors, capable of binding and sequestering cytokines and/or chemokines. To further identify host cell factors that are required for VACV infectivity we employed a multidimensional protein identification technology that incorporated HPLC and mass spectrometry, and identified 387 host proteins that associated with A4L-EGFP-VACV in the permissive PM1.CCR5 human T cell line. Among these proteins substantial numbers of them are involved in biological process that have been previously identified as critical in VACV infection. This includes proteins involved in intracellular transport $(176,291$, $513)$, cell cycle and division $(287,553,772)$, microtubule cytoskeleton organization (103, $112,205,248,250,337,597,739)$ and nucleocytoplasmic transport (Supplementary Table 4.1 and 4.2). Identification of these proteins is encouraging since it endorses the accuracy of our mass spectrometry approach.

Among the proteins that were associated with VACV 1hr p.i. was glomulin. We show that knock down of glomulin expression in PM1.CCR5 T cells reduces VACV infection. Glomulin has been shown to interact with FKBP12, an immunophilin that binds to immunosuppressive agents FK506, rapamycin and cyclosporine A $(116,152)$. These agents inhibit cell cycling. Notably, VACV infection induces the inactivation of a number of tumor suppressors, as well as the activation of pol III transcription factors, 
thereby promoting S phase cell cycle progression (772). It is intriguing to speculate that the physical association between VACV and glomulin may modulate the cell cycling machinery to promote cell cycle progression for virus replication. VACV modulation of cellular transcripts involved in cell cycle progression has been shown, namely, upregulation of cyclin A mRNA and downregulation of cyclin B, Cdk1, and Cdk2 mRNAs during the course of infection (722-724). These are key intermediates in cell cycle progression. Cyclin A upregulation has been demonstrated in tumorigenesis (726, 727) and its downregulation before $S$ phase has been shown to coincide with a delay in cellular DNA synthesis, denoting that cyclin A is crucial in DNA replication (222). This may explain the S phase arrest in VACV-infected cells. Cyclin A is also required for the G2 /M transition. Its downregulation may cause cells to either remain in G2, and not proceed to M phase (499), or to enter mitosis before the complete replication of DNA (184). The benefit of G2 / M arrest for VACV replication might be that it facilitates virus gene expression, genome replication, or morphogenesis. The advantage of a prolonged $\mathrm{S}$ phase to VACV replication is not clear. However, longer time spent in S phase may enhance virus replication, for instance, through increased cis/ trans cellular factors that facilitate poxvirus replication or by maximizing the availability of components of the host cellular DNA synthesis machinery for virus replication (741).

Another possible advantage afforded by the interaction between VACV and glomulin, is modulation of the immune response. VACV induces multiple immunoregulatory cytokines in infected cells, including transforming growth factor $\beta$ (TGF- $ß$ ) (378). TGF- $ß$ functions to drive the T helper (Th) immune response towards a Th2 response, away from the cell-mediated Th1 response effective in limiting virus 
replication (402). FKBP12 interacts with the TGF- $\beta$ type I receptor, and prevents its phosphorylation by the type II receptor in the absence of TGF- $\beta$. Thus, FKBP12 blocks ligand-independent activation of TGF- $\beta$ type II receptor signaling. Glomulin, through its interaction with FKBP12, may act as a repressor of this inhibition, sequestering FKBP12 away from the TGF- $\beta$ type I receptor, thereby permitting TGF- $\beta$ type II receptor signaling (121). Our data suggest a novel immune evasion mechanism employed by VACV to favour lineage commitment towards Th2 and away from the protective Th1 immune response.

Previous studies using the yeast two-hybrid assay have shown that glomulin associates with the intracellular portion of the proto-oncogene, c-Met, also known as hepatocyte growth factor (HGF) receptor. This receptor is a transmembrane tyrosine kinase, which is tyrosine phosphorylated upon engagement by HGF. Glomulin interacts with the inactive, non-phosphorylated form of c-MET. When c-MET is activated by HGF, glomulin is released in a phosphorylated form leading to the subsequent phosphorylation and activation of p70 S6kinase (p70S6K). Cognizant of the role of p70S6K in protein synthesis, we speculate that VACV may modulate host protein synthesis and consequently, cell division, through glomulin binding (243). We demonstrate that the use of a c-Met phosphorylation inhibitor led to a reduction in VACV infectivity. The data indicate that blockade of c-Met phosphorylation, reduced cytosolic availability of activated glomulin, which in turn led to a decrease in VACV infectivity. HGF/c-Met engagement triggers ERK activation $(324,325)$. We have previously shown that the cascade of events that leads to a permissive phenotype in PM1.CCR5 cells includes phosphorylation/activation of Jak-2, IRS-2, ERK1/ 2 and Grb2 (536). It is 
possible, therefore, that inhibiting the phosphorylation of c-Met leads to diminished ERK phosphorylation post-VACV infection, and a subsequent reduction in viral infection. Viewed altogether, these data provide evidence for the association of VACV with a large number of cell proteins. It remains unclear how specific these interactions are, or indeed, whether they all influence virus infectivity. Our ongoing investigations are directed towards characterizing many of the protein-virus associations we have identified. A better understanding of host-pathogen interactions will provide information on potential targets for therapeutic intervention strategies.

\subsection{Materials and Methods}

\subsubsection{Cell lines and viruses}

Human PM1 T cells were obtained from the National Institutes of Health AIDS Research and Reference Reagent Program and were maintained in RPMI 1640 (GibcoBRL) supplemented with $10 \% \mathrm{FCS}, 100 \mathrm{U} / \mathrm{ml}$ penicillin, $100 \mathrm{mg} / \mathrm{ml}$ streptomycin and 25 $\mu \mathrm{g} / \mathrm{ml}$ plasmocin (InvivoGen). PM1 D339 T cells were generated as previously described (536). A4L-EGFP VACV (where EGFP is enhanced green fluorescent protein) was a gift from Dr. Geoffrey Smith (Department of Virology, Faculty of Medicine, Imperial College of London)(112).A4L-EGFP VACV were grown in HeLa cells and purified by sedimentation through a $36 \%$ sucrose cushion (536), and the number of viral

particles determined by optical density at $260 \mathrm{~nm}\left(1 \mathrm{U}=1.2 \times 10^{10}\right.$ particles $)$. The particle:PFU ratio of A5L-EGFP VACV was estimated to be 50:1 


\subsubsection{In-solution protein digestion}

PM1.CCR5 and PM1.CCR5 339 cells were incubated in a hypotonic lysis buffer containing $10 \mathrm{mM}$ HEPES, pH 7.4 for 30 minutes on ice and the cell suspension briefly sonicated. The resulting lysates were incubated for 30 minutes at $4^{\circ} \mathrm{C}$ and centrifuged at $14000 \mathrm{rpm}$ for $30 \mathrm{~min}$ at $4^{\circ} \mathrm{C}$. $150 \mathrm{mg}$ of protein was aliquot from each sample was precipitated with 5 -volumes of ice-cold acetone overnight at $-20^{\circ} \mathrm{C}$, followed by centrifugation at $16,000 \mathrm{xg}$ for $15 \mathrm{~min}$. A solution of $8 \mathrm{M}$ urea, $2 \mathrm{mM}$ DTT, $50 \mathrm{mM}$ Tris$\mathrm{HCl}, \mathrm{pH} 8.5$ was used to solubilize the protein pellet for 1 hour at $37^{\circ} \mathrm{C}$, followed by carboxyamidomethylation with $10 \mathrm{mM}$ iodoacetamide for 1 hour at $37^{\circ} \mathrm{C}$ in the dark. The samples were diluted to $\sim 1.5 \mathrm{M}$ urea with $50 \mathrm{mM}$ ammonium bicarbonate, $\mathrm{pH} 8.5$ and calcium chloride was added to a final concentration of $1 \mathrm{mM}$. A 1:25 molar ratio of recombinant, proteomics grade trypsin (Roche Diagnostics, Laval, QC) was used to digest the samples at $37^{\circ} \mathrm{C}$ overnight. Solid phase-extraction of the resulting peptide mixtures was performed using Varian OMIX cartridges (Mississauga, ON, Canada) according to the manufacturer's directions. Samples were stored at $-80^{\circ} \mathrm{C}$ until MudPIT analysis.

\subsubsection{Multidimensional protein identification technology (MudPIT) analysis}

An automated 6-cycle MudPIT procedure was run similarly to previously described protocols (645) (329). A quaternary HPLC-pump was interfaced with a LTQ linear iontrap mass spectrometer (Thermo Fisher Scientific, San Jose, CA) equipped with a nanoelectrospray source (Proxeon Biosystems, Odense, Denmark). Samples were manually loaded onto a 100mm inner diameter fused silica capillary (InnovaQuartz, Phoenix, AZ) 
that had been pulled to a fine tip using a P-2000 laser puller (Sutter Instruments, Novato, CA) and packed with $\sim 7 \mathrm{~cm}$ of JupiterTM $4 \mathrm{~m}$ Proteo $90 \AA \mathrm{C} 12$ reverse phase resin (Phenomenex, Torrance, CA), followed by $\sim 5 \mathrm{~cm}$ of Luna ${ }^{\circledR} 5 \mathrm{~m}$ SCX $100 \AA$ strong cation exchange resin (Phenomenex, Torrance, CA). As peptides eluted from the microcapillary columns, they were electrosprayed directly into the MS. A distal $2.3 \mathrm{kV}$ spray voltage was applied to the microsplitter tee (Proxeon Biosystems). The MS operated in a cycle of one full-scan mass spectrum $(400-1400 \mathrm{~m} / \mathrm{z})$, followed by 6 data-dependent MS/MS spectra at $35 \%$ normalized collision energy, which was continuously repeated throughout the entire MudPIT separation. The MS functions and the HPLC solvent gradients were controlled by the Xcalibur data system (Thermo Fisher Scientific, San Jose, CA).

\subsubsection{Protein identification, validation and grouping}

Raw files were converted to m/zXML using ReAdW and searched against a human IPI (International Protein Index; http://www.ebi.ac.uk/IPI) protein sequence database (v3.20) supplemented with VACV protein sequences downloaded from (http://www.poxvirus.org/sequence.asp) and trypsin by a locally installed version of $\mathrm{X}$ !Tandem. The protein sequence database also contained all IPI protein sequences in reversed amino acid orientation (target-decoy strategy), used to estimate and minimize our false positive rate as recently described. Search parameters were: Parent ion D-mass of $4 \mathrm{Da}$, fragment mass error of $0.4 \mathrm{Da}$, and complete carbaminomethyl modification of cystein by IAA and methionine oxidation as a variable modification. Peptides passing a default log-expectation value of -1 were further evaluated (see below) (234) (645). False positive identifications were minimized using a rigorous peptide quality control strategy as recently reported. For the present study, we have set the value of total reverse spectra 
to total forward spectra to $0.5 \%$. This resulted in an empirical FDR of $<1 \%$ (8 decoy proteins compared to 1892 target proteins). The final list of identified proteins was generated from fully tryptic peptides $\geq 7$ amino acids matching these criteria. Only proteins identified with two unique peptides per analyzed sample were accepted. Two biological replicated were analyzed. A database grouping schema was developed to minimize protein inference and we report only proteins with substantial peptide information (234). Briefly, several grouping steps are performed on the database level by an algorithm applying a heuristic approach by favoring proteins within a parsimonious cluster and identified with the largest number of peptides. Similar strategies have been applied by others.

\subsubsection{Data comparison}

Comparison of our proteome data sets was achieved using the ProteinCenter bioinformatics software (Proxeon Biosystems, Odense, Denmark). To link data sets, we loaded the IPI accession keys for the 399, CCR5 and negative control samples into ProteinCenter. Individual datasets were then linked via accession keys and sequence homology (a 95\% sequence homology was required to be considered identical), to further remove any redundancy, in the ProteinCenter database.

\subsubsection{Measurements of viral infectivity}

VACV infection in different cell types was measured by assaying for vaccinia-associated ß-galactosidase activity as described previously (536).

\subsection{7. siRNA transfection}


Small interfering RNA (siRNA) duplexes were synthesized and purified according to manufacturer's protocol (QIAGEN, Inc). The glomulin target sequences were siRNA1 (5'-CUCUUGACAGGGCUCUAUU-3') and siRNA2 (5'-

AAGCCAGGACGGUCACCUU-3'). PM1.CCR5 cells $\left(10^{7}\right)$ were transfected with either a mixture of $10 \mu \mathrm{g}$ of siRNA1 and $5 \mu \mathrm{g}$ siRNA2 or scrambled control siRNA by electroporation (BTX T820 electroporator). The settings were $475 \mathrm{~V}, 1$ millisecond, and four pulses. At 48 hrs post- transfection glomulin mRNA level was evaluated using RTPCR.

\subsubsection{Reverse-transcription PCR}

PM1.CCR5 T cells were untransfected or transfected with either glomulin specific SiRNA or scrambled SiRNA, lysed 48 hrs post-transfection using the QIAGEN QIAshredder columns, and RNA isolated using the QIAGEN RNeasy minikit according to manufacturer's protocol. cDNA was synthesized from $2 \mu \mathrm{g}$ of RNA using oligo(dT) primers $\left(25^{\circ} \mathrm{C}\right.$ for $10 \mathrm{~min}, 37^{\circ} \mathrm{C}$ for $50 \mathrm{~min}$, and $70^{\circ} \mathrm{C}$ for $\left.15 \mathrm{~min}\right)$. PCR was performed using the following primer sets: (forward) 5'-TTA TCT GAT GTT GTT GTT GTT CGC3' and (reverse) 5'-GTT TAG TTC GTC GAG TGA AC-3' 
Supplementary Tables 


\section{Supplementary Table 4.1}

Gene Ontology (GO) representing the biological processes of proteins in the CCR5 specific cluster that interact with VACV. Enriched GO and phenotype ontology terms are

listed together with $p$ values and the number of proteins sorted in each category. 
Term

GO ID P value (Average) Gene Hits

protein localization

GO:0008104

GO:0045184

protein transport

GO:0015031

GO:0046907

intracellular transport

RNA processing

DNA metabolic process

intracellular protein transport

carboxylic acid metabolic process

$M$ phase

mitosis

$M$ phase of mitotic cell cycle

GO:0006396

GO:0006259

GO:0006886

GO:0019752

GO:0000279

GO:0007067

GO:0000087

GO:0016071

mRNA metabolic process

mitotic cell cycle

cell division

mRNA processing

intracellular protein transport across a membrane

RNA splicing

DNA replication

alcohol metabolic process

RNA transport

mRNA transport

lipid biosynthetic process

ribosome biogenesis and assembly

fatty acid metabolic process

generation of precursor metabolites and energy

amino acid metabolic process

nucleocytoplasmic transport

nuclear transport

translational initiation

microtubule cytoskeleton organization and biogenesis

regulation of translation

RNA splicing, via transesterification reactions

nuclear mRNA splicing, via spliceosome

cholesterol metabolic process

sterol metabolic process

DNA-dependent DNA replication

cholesterol biosynthetic process

transcription from RNA polymerase III promoter

sterol biosynthetic process

nuclear export

chromosome segregation

steroid biosynthetic process

phosphoinositide-mediated signaling

Golgi vesicle transport

spindle organization and biogenesis

spliceosome assembly

ER to Golgi vesicle-mediated transport

regulation of cyclin-dependent protein kinase activity

RNA export from nucleus

androgen receptor signaling pathway

regulation of translational initiation

rRNA processing

transcription initiation

rRNA metabolic process

glucose catabolic process

steroid hormone receptor signaling pathway

GO:0000278

GO:0051301

GO:0006397

GO:0065002

GO:0008380

GO:0006260

GO:0006066

GO:0050658

GO:0051028

GO:0008610

GO:0042254

GO:0006631

GO:0006091

GO:0006520

GO:0006913

GO:0051169

GO:0006413

GO:0000226

GO:0006417

GO:0000375

GO:0000398

GO:0008203

GO:0016125

GO:0006261

GO:0006695

GO:0006383

GO:0016126

GO:0051168

GO:0007059

GO:0006694

GO:0048015

GO:0048193

GO:0007051

GO:0000245

GO:0006888

GO:0000079

GO:0006405

GO:0030521

GO:0006446

GO:0006364

GO:0006352

GO:0016072

GO:0006007

GO:0030518

\begin{tabular}{|c|c|}
\hline 0 & 42 \\
\hline 0 & 40 \\
\hline 0 & 39 \\
\hline 0.000043 & 32 \\
\hline 0.000067 & 23 \\
\hline 0.000962 & 23 \\
\hline 0.000053 & 21 \\
\hline 0.002058 & 19 \\
\hline 0.000258 & 17 \\
\hline 0.000067 & 16 \\
\hline 0.000074 & 16 \\
\hline 0.001052 & 16 \\
\hline 0.002844 & 16 \\
\hline 0.000574 & 15 \\
\hline 0.000666 & 15 \\
\hline 0 & 14 \\
\hline 0.00038 & 14 \\
\hline 0.001338 & 13 \\
\hline 0.00786 & 13 \\
\hline 0.000002 & 12 \\
\hline 0.000002 & 11 \\
\hline 0.01714 & 11 \\
\hline 0.000041 & 9 \\
\hline 0.005679 & 9 \\
\hline 0.021913 & 9 \\
\hline 0.038925 & 9 \\
\hline 0.008415 & 8 \\
\hline 0.009122 & 8 \\
\hline 0.000474 & 7 \\
\hline 0.005325 & 7 \\
\hline 0.009527 & 7 \\
\hline 0.004362 & 6 \\
\hline 0.004362 & 6 \\
\hline 0.008349 & 6 \\
\hline 0.011429 & 6 \\
\hline 0.014645 & 6 \\
\hline 0.000265 & 5 \\
\hline 0.000538 & 5 \\
\hline 0.000538 & 5 \\
\hline 0.00423 & 5 \\
\hline 0.016637 & 5 \\
\hline 0.018499 & 5 \\
\hline 0.030228 & 5 \\
\hline 0.032947 & 5 \\
\hline 0.001349 & 4 \\
\hline 0.003971 & 4 \\
\hline 0.004373 & 4 \\
\hline 0.006798 & 4 \\
\hline 0.008183 & 4 \\
\hline 0.00861 & 4 \\
\hline 0.015846 & 4 \\
\hline 0.01963 & 4 \\
\hline 0.020751 & 4 \\
\hline 0.021133 & 4 \\
\hline 0.039463 & 4 \\
\hline 0.047706 & 4 \\
\hline
\end{tabular}




\section{Supplementary Table 4.2}

GO representing the molecular function of proteins in the CCR5 specific cluster.

Enriched GO and phenotype ontology terms are listed together with $p$ values and the number of proteins sorted in each category. 


\begin{tabular}{l} 
Term \\
\hline RNA binding \\
GTP binding \\
guanyl nucleotide binding \\
translation factor activity, nucleic acid binding \\
translation regulator activity \\
helicase activity \\
GTPase activity \\
translation initiation factor activity \\
coenzyme binding \\
nuclease activity \\
endonuclease activity \\
single-stranded DNA binding \\
heat shock protein binding \\
nuclear hormone receptor binding \\
oxidoreductase activity, NAD or NADP as acceptor \\
protein transporter activity \\
RNA helicase activity \\
transferase activity, transferring pentosyl groups
\end{tabular}

GO ID

GO:0003723

GO:0005525

GO:0019001

GO:0008135

GO:0045182

GO:0004386

GO:0003924

GO:0003743

GO:0050662

GO:0004518

GO:0004519

GO:0003697

GO:0031072

GO:0035257

GO:0016616

GO:0008565

GO:0003724

GO:0016763
P value (Average) Gene Hits

$\begin{array}{rr}0 & 42 \\ 0.002368 & 16 \\ 0.002844 & 16 \\ 0.00011 & 10 \\ 0.000429 & 10 \\ 0.002518 & 10 \\ 0.018713 & 9 \\ 0.000065 & 8 \\ 0.011385 & 8 \\ 0.035647 & 7 \\ 0.011759 & 6 \\ 0.002992 & 5 \\ 0.003662 & 5 \\ 0.010255 & 5 \\ 0.025905 & 5 \\ 0.034157 & 5 \\ 0.003243 & 4 \\ 0.017848 & 4\end{array}$




\section{Chapter 5}

\section{Discussion and Future Directions}




\subsection{Discussion}

Post 9/11/2001 there has been a growing concern that biologic weapons might be deployed in warfare or for terrorist purposes. Declared eradicated in 1980, smallpox was considered an unlikely agent of bioterrorism because of the high level of population immunity to the disease, the availability of a vaccine and the knowledge that vaccination of patients' immediate contacts could rapidly control outbreaks. However, all vaccination against smallpox ceased after $1980(194,747)$ and vaccination only provides a high level of immunity for about 3 to 5 years, decreasing thereafter. Therefore, virtually all children and many adults are now fully susceptible to smallpox and only limited supplies of vaccine $(\mathrm{VACV})$ are available. While VARV, the causative agent for smallpox, has a narrow natural host range in humans, with non-human primates exhibiting susceptibility in a laboratory setting (93) and VACV exhibits a broader range of infectible hosts, intranasal infection (the physiologically relevant route of inoculation) with either virus results in similar dissemination: replication occurs initially in lymph nodes draining the upper respiratory tract, after which a transient viremia spreads virus to reticuloendothelial cells throughout the host leading to systemic infection with considerable lethality at high doses of virus. In VARV, a second round of replication within these sites precedes a more intense secondary viremia and the subsequent clinical manifestations of the disease. Based on pathogenic similarities, VACV presents as a suitable model for VARV infection $(85,259,267,270,540)$. The objective of the studies described herein was to characterize host-response factors, at the cellular level, that determine and define susceptibility to infectivity. A clear understanding of how an infecting poxvirus renders a cell permissive for infection and replication and the mechanism whereby systemic 
dissemination occurs would likely reveal potential targets for therapeutic drug development.

\subsubsection{In vitro $\mathrm{VACV}$ infection}

In recent years accumulating evidence suggests that poxviral replication requires the manipulation of target cells in the context of activation of signaling pathways (314, 416). In chapter 2 we provide evidence for VACV activation of the chemokine receptor, CCR5, and the phosphorylation-activation of a number of signaling effectors. Our data provide the first evidence of a direct link between virus-induced receptor activation and viral replication. Specifically, we identify a critical intracellular tyrosine residue in CCR5 that confers susceptibility to viral infection. Our results are consistent with a significant role for the induced activation of tyrosine kinases for productive vaccinia virus infection. These findings set the stage for antiviral strategies based on interventions associated with limiting CCR5 activation and signaling.

There has been much controversy in the field regarding the mechanisms by which poxviruses enter target cells. In an earlier publication our group reported that for another poxvirus, myxoma virus, infection rapidly activates tyrosine kinase signal transduction in permissive cells (416). We implicated the cell surface receptor CCR5 in mediating these phosphorylation events in the cells we studied. Our working hypothesis was that the poxvirus engages CCR5-mediated signaling events to create the cellular environment that is hospitable for viral replication. In a subsequent publication, Johnston et. al identified a role for the serine-threonine kinase, PAK-1, in myxoma virus replication, and suggested that the permissiveness of target cells to infection resides in their ability to activate this kinase (314). We do not contest that activation of PAK-1 may be a determinant for 
permissiveness to infection. The critical issue is how is this cellular kinase activated? Our data suggest that VACV is capable of engaging CCR5 to activate signaling events that are required for viral replication. We provide definitive experimental results to support this, namely knockdown of ectopic CCR5 expression that leads to restricted/reduced viral infection, and introduction of CCR5 into non-permissive cells that renders them susceptible to infection. Additionally, we have gone on to characterize the specific intracellular tyrosine residues in CCR5 that apparently are critical for permissive infection that is mediated by CCR5. Phosphorylation of the CCR5 Y-339 receptor residue may be an early event in a virus-induced signaling cascade that accompanies infection and Y-339 may be responsible for recruitment of signaling intermediates that we have shown to be induced by VACV early post infection. Subsequent activation of crucial downstream kinases such as PAK-1 and other serine/threonine kinases may, in turn, promote some early aspect of viral replication. Our findings suggest that viral activation of CCR5 activates signaling events that involve tyrosine phosphorylation, thereby rendering a cell permissive for viral replication.

Our confocal studies support these findings, namely that virus entry into cells is not mediated by CCR5. Indeed, our data demonstrate that poxviruses enter both permissive and non-permissive PM1 T cell lines, and that a post-entry event determines whether viral replication ensues. Our data in primary human $\mathrm{T}$ cells are in agreement with other published data, namely that ectopic expression of a receptor that is inducibly expressed in activated $\mathrm{T}$ cells confers permissiveness for vaccinia virus infection (115). Certainly, activation of resting T cells results in de novo expression of CCR5, as was discussed in Chapter 2. Our data suggest that virus engagement of CCR5 will permit 
replication to proceed. Certainly, cell types that do not express CCR5 are also permissive for infection, thus we infer that poxviruses may activate different receptors to mediate the sequence of events that promote viral replication. The challenge is to determine the mechanism whereby the virus will activate a receptor and to identify the signaling effectors that predispose a cell for viral replication. Moreover, it has been suggested that an IFN response alone defines the permissive phenotype of a cell for the poxvirus, myxoma virus (725). Unquestionably, the ability of a cell to mount an effective IFN response to virus infection will determine the extent of viral replication in the target tissue. A priori, our data indicate that permissiveness for viral replication requires receptor-mediated activation of signaling effectors. That the virus-induced IFN response may inhibit this viral replication is a separate issue.

Although VACV infects a variety of cells, some poxviruses fail to grow in certain host cells in vitro, a phenomenon known as host restriction $(173,174,192,215,286,288$, $421,670,763)$. In these restrictive cells, poxviruses enter the cell efficiently but fail to replicate. This phenomenon is a result of either the inability to circumvent innate intracellular barriers, or the failure of the cell to provide complementing factors necessary for vial replication $(423,536)$. Consequently, the ability of poxviruses to manipulate signal transduction networks of infected cells has a major impact on the outcome of infection. The distinct tropism of each poxvirus is therefore strictly dependent on a unique repertoire of host range (HR) genes utilized by the virus. The dynamic struggle between HR proteins and antiviral pathways can determine whether a specific infection will be permissive or not (611). In order to appreciate the sophisticated interplay between viral and cellular proteins, the role HR factors play in manipulating host intracellular 
signaling pathways must be better characterized.

In Chapter 2 we provided evidence that in non-permissive cells, viral replication is blocked in late gene expression. We then showed the co-localization of VACV and CCR5 in permissive cells. We hypothesize that this co-localization results in activation of signaling effectors necessary for viral replication either through direct or indiect recruitment of the signaling effector to the intracellular region of CCR5. CCR5 activation leads to a cascade of tyrosine phosphorylation signaling events necessary for late VACV gene expression. Other lines of evidence indicate a role for other host range cellular components in the VACV replication cycle. Notably, it has been shown that viral intermediate-stage transcription factor 2 is a cellular protein necessary for a successful VACV infection (569).

Employing multidimensional protein identification technology (MudPIT) analysis, in Chapter 4 we show that VACV interacts with a large subset of cellular proteins. These interactions likely serve to influence VACV infection, by either promoting viral replication or inhibiting the induction of antiviral activity. We identified glomulin as a novel host derived protein involved in productive replication of VACV in the permissive human T cell line, PM1.CCR5. We show that knocking down glomulin expression in PM1.CCR5 T cells significantly reduces VACV infection. Glomulin has been described to interact with FKBP12, an immunophilin that binds to immunosuppressive agents FK506, rapamycin and cyclosporine A $(116,152)$. These agents are inhibitors of cell cycling. Furthermore, VACV infection induces the inactivation of major tumor suppressors, as well as the activation of pol III transcription factors, therefore promoting host cell cycle progression into S phase (772). It is intriguing to speculate that the 
physical interaction between VACV and glomulin may modulate the cell cycling machinery to enhance viral replication. VACV modulation of cellular transcripts involved in cell cycle progression has been shown; namely upregulation of cyclin A mRNA and downregulation of cyclin B, Cdk1, and Cdk2 mRNAs during the course of infection (722-724). Cyclin A upregulation occurs in tumorigenesis $(726,727)$ and its downregulation before S phase coincides with a delay in cellular DNA synthesis, suggesting that cyclin A is crucial in DNA replication (222). This may explain the $\mathrm{S}$ phase arrest in VACV-infected cells. Cyclin A is also required for the G2 /M transition. Its downregulation may cause cells to either remain in $\mathrm{G} 2$, and not proceed to M phase (499), or to enter mitosis before the complete replication of DNA (184). The benefit of G2 / M arrest for VACV replication might be that it facilitates virus gene expression, genome replication, or morphogenesis. The advantage of a prolonged S phase to VACV replication is not clear. However, longer time spent in S phase may enhance virus replication, for instance, through increased cis/ trans cellular factors that facilitate poxvirus replication or by maximizing the availability of components of host cellular DNA synthesis machinery for virus replication (741). We further show that a c-Met phosphorylation inhibitor led to a reduction in VACV infectivity. The data indicate that blockade of c-Met phosphorylation reduced cytosolic availability of activated glomulin, which in turn led to a decrease in VACV infectivity.

Among signaling effectors that seem to be crucial for productive VACV replication are the adaptor proteins Grb2 and Erk1/2. Grb2 activation may have very specific implications for VACV infection, especially since its phosphorylation has been shown to reduce SH3-dependent binding to Sos (369) and is thus thought to represent a 
potential regulatory point for signal transduction that may regulate the specificity of SH3 domain interactions (66). Grb2 participates in the Ras signaling cascade, associating with the guanine nucleotide exchange factor Sos, and thus may link signaling effectors to the MAP kinase pathway. This activation of the MAPK pathway appears to be virus-specific, since U0126, a MAPK/Erk1,2 inhibitor, has been shown to attenuate VACV infection, but increase myxoma virus replication in permissive $3 \mathrm{~T} 3$ cell lines (314). It has also been demonstrated that $\mathrm{HGF} / \mathrm{c}-$ Met engagement triggers ERK activation $(324,325)$. We speculate that inhibiting the phosphorylation of c-Met leads to diminished ERK phosphorylation post-VACV infection, and therefore a subsequent reduction in viral infection.

Taken together, the results from Chapters 2 and 4 indicate that cell tropism / infectivity depends on the ability of the virus to manipulate signaling pathways to its advantage. The data acquired from the mass spectrometry studies in Chapter 4 may provide additional cell proteins that are targets for productive VACV infection, and are the subject of ongoing investigations.

\subsubsection{In vivo $\mathrm{VACV}$ infection}

The objective of the in vivo studies presented in Chapter 3 was to investigate the mechanism of viral dissemination in a respiratory infection model of VACV. We employed VACV as a surrogate for smallpox and the mouse model to recapitulate the human disease. In this chapter we provide several lines of evidence demonstrating the importance of CCR5 expression for VACV dissemination in a mouse model of infection. Our data provide the first evidence for the requirement of $\mathrm{CCR}^{+} \mathrm{T}$ cells in VACV 
dissemination from the initial site of inoculation, the respiratory tract, to visceral tissue (535). These experiments were a natural extension of our in vitro findings, which revealed that VACV could engage CCR5 to activate signaling events that are required for viral replication (536). We provided evidence that virus activation of CCR5 in T cells allows for replication to proceed. Based on studies that indicated that permissive infection of $\mathrm{T}$ cells could represent a mechanism for virus dissemination throughout the lymphatic system (466), we hypothesized that the absence of CCR5 might be protective for VACV systemic infection. Given the current interest surrounding bioterrorism and the threat of smallpox as a potential biologic weapon, these data provide further novel insights into the pathogenesis of poxviruses, specifically in the context of immune cells. Although not within the scope of these studies, the significance of our findings resides in the potential to develop CCR5 antagonists as antivirals against poxviruses.

In addition to activated T cells, DCs and macrophages also express CCR5 (77, $211,260,331,541,554,588)$. We evaluated DC and macrophage infection by VACV in the BAL fluid of $\mathrm{CCR}^{+/+}$and $\mathrm{CCR} 5^{-/}$mice post VACV infection, and we report infection in these cell types at different time points post infection. However, it was previously shown that VACV undergoes abortive replication in DCs (181) and induces apoptotic cell death in both DCs and macrophages $(181,293)$, whereas productive infection occurs in activated, but not resting T cells (115). In addition, the productive infection of a subpopulation of activated $\mathrm{T}$ cells might account for dissemination of the VACV away from local sites of inoculation (770), herein supported by our data. Moreover, it was proposed that the potential for VACV to disseminate in $\mathrm{T}$ cells of the memory phenotype may have relevance to the ability for VACV infection to persist in the 
host (775). Certainly, cell types that do not express CCR5 are also permissive for infection, thus we infer that poxviruses may activate different receptors to mediate the sequence of events that promote viral replication. The challenge is to determine the mechanisms whereby the virus will activate a receptor and to identify the signaling effectors that predispose a cell for viral replication and further dissemination in vivo. In a recent publication, data are provided that demonstrate that subcutaneous injection of TAK-779, a CCR5 antagonist, in a mouse model of asthma resulted in significantly diminished pulmonary inflammation, the analyses focusing on BAL and lung tissue (661). Expression of CCR5 in total lung cell suspensions was reduced in the TAK-779 treated mice compared to untreated mice. Additionally, the number of immune cells in affected lungs, in mice treated with TAK-779, was also reduced. These findings are consistent with TAK-779 treatment disabling CCR5 signaling such that chemokinemediated cell trafficking is inhibited (770). In our study, administration of TAK-779 reduced viral dissemination to the spleens of $\mathrm{CCR}^{+/+}$mice, with no effect on viral lung titers. Since TAK-779 inhibits the migration of leukocytes, including T cells, and abrogates CCR5-inducible signaling in vivo, we speculate that a TAK-779 mediated reduction of CCR5-positive $\mathrm{T}$ cell trafficking to the primary site of infection, the lungs, and TAK-779 inhibition of CCR5-mediated signaling in the T cells, required for VACV replication $(633,770)$, may be reflected in the subsequent reduction of VACV dissemination to the spleen. Certainly TAK-779 administered to the CCR ${ }^{+/+}$mice may not be sufficient to completely inhibit all expressed CCR5 and may allow for residual viral replication in the lung T cells. In addition, given that VACV infects cells of different lineages, including those that may not express CCR5, we might expect viral 
replication to be unaffected in some cell types.

The possibility that the differences between VACV infection in $\mathrm{CCR} 5^{+/+}$ compared to $\mathrm{CCR} 5^{-/-}$are due to disruption of the action of one or more of the ligands for CCR5 is not the case. Although several strains of VACV express a chemokine/ligand binding inhibitor to subvert the host immune response, the Western Reserve (used in these studies) and Copenhagen strains do not encode chemokine inhibitors (545). Accordingly, based on the absence of virally-encoded ligand inhibitors for CCR5, cell recruitment into the lungs is unaffected in the $\mathrm{CCR} 5^{+/+}$mice infected with this strain of VACV. In addition, lung leukocyte recruitment - trafficking that would be chemokinemediated - is comparable in $\mathrm{CCR}^{+/+}$and $\mathrm{CCR} 5^{-/}$mice post influenza A/WSN/33 infection, albeit not VACV, in agreement with other reports (734).

In a final series of experiments we showed that adoptive transfer of $\mathrm{CCR} 5^{+}$cells into recipient $\mathrm{CCR}^{-/-}$mice confers an increase in permissiveness to VACV infection. Our analyses reveal that whereas there are differences in virus titers in lungs, BAL and spleens of $\mathrm{CCR}^{+/+}$and $\mathrm{CCR}^{-/-}$mice, and differences in absolute cell counts in their $\mathrm{BAL}$ and dLNs, once $\mathrm{CCR} 5^{+}$cells are adoptively transferred into the $\mathrm{CCR} 5^{-/}$mice these differences are diminished, and supported by the statistical analyses performed. Given that a sub-lethal dose of VACV was employed, the biological significance of the results resides in two findings: (1) that there is an accumulation of CD4 and CD8 cells into sites of infection in all recipient mice, and (2) that there is detectable virus in the spleens of $\mathrm{CCR}^{-/ /}$mice that are recipients of CCR5+ leukocytes. Since (i) a robust $\mathrm{T}$ cell response, associated with activated VACV-specific CD8 $+\mathrm{T}$ cells and $\mathrm{CD} 4+\mathrm{T}$ cells is elicited post efficient $\mathrm{VACV}$ infection in mice $(256,544)$ and (ii) productive infection of a 
subpopulation of activated $\mathrm{T}$ cells, at the site of infection, may account for dissemination of the virus away from local sites of inoculation (49), these findings further highlight the importance of CCR5 in VACV dissemination in vivo.

Taken together, our data in Chapter 3 identify that the contributions of CCR5 activation to VACV infection are not redundant and suggest that CCR5 may be required for systemic VACV infection in vivo.

\subsection{Future Directions}

\subsubsection{Characterization the role of WASp in VACV infection}

As discussed in Chapter 1, WASp has been implicated in host cellular actin polymerization invoked by VACV $(204,205,597)$. Specifically, data obtained by analysis of VACV infected Hela cells suggest that Src-family-mediated tyrosine phosphorylation of the VACV transmembrane protein A36R enables recruitment of WIP, Nck and Grb2 to the viral particle, with consequent formation of an actin-tail complex and motility of the virus to the plasma membrane(597). These data do not consider the relevance of WASp/N-WASp to VACV pathogenicity beyond a major role for N-WASp in linking VACV infection to host cell cytoskeletal changes. Accordingly, future studies will focus on whether VACV infection mediates direct/indirect interactions of WASp with effectors such as Fyn and Nck, and CCR5. The possibility that VACV activation of CCR5 triggers WASp interactions with other cell effectors will be studied by immunoprecipitation of WASp from VACV infected PM1.CCR5 cells, then applying mass spectrometry as described in Chapter 4, to distinguish WASp interacting proteins. 


\subsubsection{VACV regulation of host cell gene expression}

The data in Chapter 2 indicate that VACV activation of CCR5 initiates the rapid phosphorylation of signaling effectors that are intermediates in signaling cascades that have the potential to regulate a number of biological responses. To gain a better understanding of how VACV-mediated activation of CCR5 results in a host cell environment that is permissive for viral replication, we propose to undertake studies to identify host cell genes that are regulated by the virus. In virus dose-response time course studies, we will examine the effects of VACV infection of PM1, PM1.CCR5 and PM1.CCR5Y3F T cells on gene expression profiles, using the Affymetrix U133 Plus 2.0 human gene set microarray. Genes that are differentially expressed - either upregulated or downregulated - in the permissive PM1.CCR5 cells compared with the non-permissive PM1 and PM1CCR5Y3F cells, will be identified, validated by quantitative real-time PCR, and clustered according to their potential function (e.g. kinase, adaptor molecule). These studies will elucidate the contribution of CCR5-inducible signaling to VACV infectivity, focusing on different stages of the virus replicative cycle.

\subsubsection{Examination of the antiviral effects of IFNs}

To examine the contribution of an IFN response to the course of VACV infection, we propose experiments to examine the virus-inducible IFN profile in permissive PM1.CCR5 T cells. Specifically, cells will be infected with VACV, then at $2 \mathrm{hr}, 6 \mathrm{hr}$, $8 \mathrm{hr}$, $12 \mathrm{hr}$ and $24 \mathrm{hr}$ p.i. virus infected cells will be lysed, RNA harvested and IFN-specific transcripts probed with human IFN- $\beta$ and IFN- $\alpha$ primer sets. We have developed a qRTPCR assay for the different human IFN- $\alpha$ subtypes and IFN- $\beta$. Because of the high 
degree of amino acid identity among the different IFN- $\alpha$ subtypes, there are no antibodies that distinguish the different subtypes, hence this approach to profile the IFN signature by gene expression. In parallel, we will examine the IFN response in the VACV infected, non-permissive PM1 and PM1.CCR5Y339F cells. In PM1.CCR5Y339F cells there is a deficiency in signaling, rendering these cells resistant to VACV infection. Induction of antiviral effectors via IFN signaling or PAMP recognition is critical to restricting viral replication. It is possible that VACV engagement of CCR5-inducible signaling effectors negatively regulates (i) IFN induction and/or (ii) sensitivity to IFN. For example, VACV-mediated CCR5 signaling may activate those phosphatases that inhibit STAT or IRF localization to the nucleus, thereby limiting an IFN response. To address this, we will undertake experiments that examine whether an IFN response is blunted in permissive versus non-permissive PM1 cells, specifically focusing on IFNinducible phosphorylation-activation of signaling effectors such as STAT1, STAT2, STAT3, p38 MAPK, 4E-BP1, the p65 subunit of PI-3K. In addition, we will compare the signature gene expression profiles for an IFN response in the 3 PM1 cell types, focusing on IFN-inducible 2'5' OAS, PKR and ISG15.

\subsubsection{Characterization of specific VACV factors that bind to intracellular targets}

Our confocal studies indicate that VACV migrates towards internalized CCR5 in permissive cells expressing an intact CCR5. Apparently, subsequent tyrosine phosphorylation of the receptor and downstream effectors is crucial for viral replication. Our mass spectrometry analysis also confirmed a physical interaction between VACV and intracellular proteins. The sequence of events and cellular effectors that determine 
the translocation of the virus and possible viral proteins association with CCR5 or other intracellular agents are unknown. To confirm potential interactions between VACV and host cell proteins we could undertake two different approaches: (1) test each pair-wise combination of VACV ORF and host proteins in a yeast two-hybrid assay utilizing a protein array (697). The array consists of two sets of yeast transformants, one expressing VACV ORF as a hybrid protein with the Gal4 activation domain, which will be mated to yeast transformants of the opposite mating type carrying one of the host proteins as a hybrid protein with the Gal4 DNA-binding domain. The diploids will be placed in medium selective for the two-hybrid reporter genes, where a single protein could be tested for interaction with each VACV protein in the array. (2) Since the genome of VACV is sequenced (229) and the 266 viral ORFs are known, each single recombinant tagged-VACV protein could be expressed in E.coli . Briefly, the glutathione-Stransferase (GST) fusion proteins will be expressed in E-coli transformed by the corresponding pGEX vectors. The extracted VACV recombinant proteins will be used for binding to ${ }^{35}$ S-radiolabeled host cell proteins that are prepared in vitro using a phage RNA polymerase-coupled transcription/translation kit. Following the identification of proteins, which interact with individual VACV proteins, their biological function will be considered in the context of the VACV protein they associate with and its role in virus replication.

Our MudPIT analysis, presented in Chapter 4, also identified 387 unique proteins that physically associate with A4L-EGFP-VACV in infected PM1.CCR5 T cells. Among the 387 proteins in the PM1.CCR5-unique cluster, glomulin attracted our attention based on its ability to associate with a transmembrane tyrosine kinase receptor, become 
phosphorylated and influence downstream signaling associated with protein translation. Our data identified a role for glomulin in permissiveness of cells for VACV infection. Our analysis identified another 386 proteins that potentially interact with VACV, many of which have been previously implicated in VACV infectivity. Schlafen 11 is one such protein. Unpublished microarray data from our lab reveal that mouse embryonic fibroblast cells (MEFs) exposed to IFN treatment upregulate expression for several genes in the Schlafen (Slfn) family. We infer that these slfn proteins have a role in an IFNinduced antiviral response, thus the ability of VACV to sequester slfn 11 may limit an IFN reponse.

A poorly characterized family, the Slfn proteins have been shown to be involved in inhibition of cell cycle progression ('schlafen' is a German for 'sleep'). In vivo, mouse slfns (mslfn) inhibit T-cell development $(217,606)$, while in vitro, $\mathrm{m}$-slfn1 has been shown to cause arrest of fibroblast growth by Slfn-1-mediated inhibition of Cyclin D1 transcription, which in turn causes $\mathrm{G} 1$ arrest $(83,217,606)$. The amino acid sequences for the Slfn proteins are unlike other characterized proteins, which led to the hypothesis that they must participate in a novel cell growth regulatory mechanism. Additionally, the expression levels of m-slfns is regulated differentially after infection with the intracellular pathogens Brucella (185) and Listeria (217), suggesting a role in host defense against pathogens. In another study, recombinant VACV expressing v-slfn-exhibit an attenuated phenotype in vivo, characterized by a significantly reduced virus titre in the lungs by day 7 p.i. compared to wt VACV (247). The study suggested that expression of v-slfn does not prevent the establishment of infection or virus replication, but probably accelerates virus clearance by the immune system. 
To examine the potential contribution of host Slfn proteins to limiting VACV infection, we will employ a similar approach to that presented in chapter 4, namely an in vitro VACV infection assay in combination with siRNA-mediated knockdown of Slfns, as well as analysis of potential receptor and signaling effectors involved in slfn signaling.

In similar studies, other proteins among the 387 identified will be characterized. 


\section{Chapter 6}

Dissemination of Work Arising from this Thesis 


\section{A portion of Chapter 1 was published as:}

Galligan C.L., Murooka, T.T., Rahbar, R., Baig, E., Majchrzak-Kita, B., and Fish, E.N. (2006). Interferons and viruses: signalling for supremacy. Immunol Res 35, 27-40.

\section{Chapter 2 was published as:}

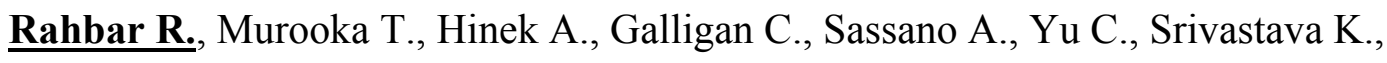
Platanias L. and Fish E.N. Vaccinia virus activation of CCR5 invokes tyrosine phosphorylation signaling events that support virus replication. J Virol. 2006 Jul;80(14):7245-59.

\section{Chapter 3 was published as:}

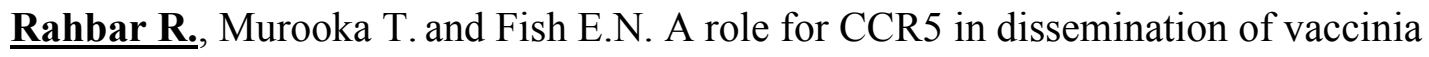
virus in vivo. J Virol. 2009 Mar;83(5):2226-36. Epub 2008 Dec 10.

\section{Chapter 4 is a manuscript submitted as:}

Rahbar R., Murooka T., Kislinger T. and Fish E.N. (2009). Glomulin, a new protein involved in VACV infection 
References 
1. CDC. Newly licensed smallpox vaccine to replace old smallpox vaccine, $p$. 207-8. In H. a. H. Services (ed.), vol. 57, Washington.

2. Aaronson, D. S., and C. M. Horvath. 2002. A road map for those who don't know JAK-STAT. Science 296:1653-5.

3. Afonso, C. L., E. R. Tulman, Z. Lu, L. Zsak, G. F. Kutish, and D. L. Rock. 2000. The genome of fowlpox virus. J Virol 74:3815-31.

4. Afonso, C. L., E. R. Tulman, Z. Lu, L. Zsak, F. A. Osorio, C. Balinsky, G. F. Kutish, and D. L. Rock. 2002. The genome of swinepox virus. J Virol 76:78390.

5. Ahn, K., T. H. Meyer, S. Uebel, P. Sempe, H. Djaballah, Y. Yang, P. A. Peterson, K. Fruh, and R. Tampe. 1996. Molecular mechanism and species specificity of TAP inhibition by herpes simplex virus ICP47. EMBO J 15:324755.

6. Aizawa, Y., K. Akita, M. Taniai, K. Torigoe, T. Mori, Y. Nishida, S. Ushio, Y. Nukada, T. Tanimoto, H. Ikegami, M. Ikeda, and M. Kurimoto. 1999. Cloning and expression of interleukin-18 binding protein. FEBS Lett 445:338-42.

7. Al-Mohanna, F., R. Parhar, and G. J. Kotwal. 2001. Vaccinia virus complement control protein is capable of protecting xenoendothelial cells from antibody binding and killing by human complement and cytotoxic cells. Transplantation 71:796-801.

8. al-Mughales, J., T. H. Blyth, J. A. Hunter, and P. C. Wilkinson. 1996. The chemoattractant activity of rheumatoid synovial fluid for human lymphocytes is due to multiple cytokines. Clin Exp Immunol 106:230-6.

9. Alcami, A. 2007. New insights into the subversion of the chemokine system by poxviruses. Eur J Immunol 37:880-3.

10. Alcami, A. 2003. Structural basis of the herpesvirus M3-chemokine interaction. Trends Microbiol 11:191-2.

11. Alcami, A. 2003. Viral mimicry of cytokines, chemokines and their receptors. Nat Rev Immunol 3:36-50.

12. Alcami, A., A. Khanna, N. L. Paul, and G. L. Smith. 1999. Vaccinia virus strains Lister, USSR and Evans express soluble and cell-surface tumour necrosis factor receptors. J Gen Virol 80 ( Pt 4):949-59.

13. Alcami, A., and U. H. Koszinowski. 2000. Viral mechanisms of immune evasion. Trends Microbiol 8:410-8.

14. Alcami, A., and G. L. Smith. 1992. A soluble receptor for interleukin-1 beta encoded by vaccinia virus: a novel mechanism of virus modulation of the host response to infection. Cell 71:153-67.

15. Alcami, A., and G. L. Smith. 2002. The vaccinia virus soluble interferongamma receptor is a homodimer. J Gen Virol 83:545-9.

16. Alcami, A., and G. L. Smith. 1995. Vaccinia, cowpox, and camelpox viruses encode soluble gamma interferon receptors with novel broad species specificity. J Virol 69:4633-9. 
17. Alcami, A., J. A. Symons, P. D. Collins, T. J. Williams, and G. L. Smith. 1998. Blockade of chemokine activity by a soluble chemokine binding protein from vaccinia virus. J Immunol 160:624-33.

18. Ali, S., L. A. Hardy, and J. A. Kirby. 2003. Transplant immunobiology: a crucial role for heparan sulfate glycosaminoglycans? Transplantation 75:1773-82.

19. Ali, S., A. C. Palmer, B. Banerjee, S. J. Fritchley, and J. A. Kirby. 2000. Examination of the function of RANTES, MIP-1alpha, and MIP-1beta following interaction with heparin-like glycosaminoglycans. J Biol Chem 275:11721-7.

20. Alkhatib, G., C. Combadiere, C. C. Broder, Y. Feng, P. E. Kennedy, P. M. Murphy, and E. A. Berger. 1996. CC CKR5: a RANTES, MIP-1alpha, MIP1 beta receptor as a fusion cofactor for macrophage-tropic HIV-1. Science 272:1955-8.

21. Allen, S. J., S. E. Crown, and T. M. Handel. 2007. Chemokine: receptor structure, interactions, and antagonism. Annu Rev Immunol 25:787-820.

22. Amara, R. R., P. Nigam, S. Sharma, J. Liu, and V. Bostik. 2004. Long-lived poxvirus immunity, robust CD4 help, and better persistence of CD4 than CD8 T cells. J Virol 78:3811-6.

23. Anderson, J. B., S. A. Smith, and G. J. Kotwal. 2002. Vaccinia virus complement control protein inhibits hyperacute xenorejection. Transplant Proc 34:1083-5.

24. Andrade, A. A., P. N. Silva, A. C. Pereira, L. P. De Sousa, P. C. Ferreira, R. T. Gazzinelli, E. G. Kroon, C. Ropert, and C. A. Bonjardim. 2004. The vaccinia virus-stimulated mitogen-activated protein kinase (MAPK) pathway is required for virus multiplication. Biochem J 381:437-46.

25. Andres, P. G., P. L. Beck, E. Mizoguchi, A. Mizoguchi, A. K. Bhan, T. Dawson, W. A. Kuziel, N. Maeda, R. P. MacDermott, D. K. Podolsky, and H. C. Reinecker. 2000. Mice with a selective deletion of the CC chemokine receptors 5 or 2 are protected from dextran sodium sulfate-mediated colitis: lack of CC chemokine receptor 5 expression results in a NK1.1+ lymphocyteassociated Th2-type immune response in the intestine. J Immunol 164:630312.

26. Angers, S., A. Salahpour, and M. Bouvier. 2002. Dimerization: an emerging concept for $\mathrm{G}$ protein-coupled receptor ontogeny and function. Annu Rev Pharmacol Toxicol 42:409-35.

27. Angers, S., A. Salahpour, E. Joly, S. Hilairet, D. Chelsky, M. Dennis, and M. Bouvier. 2000. Detection of beta 2-adrenergic receptor dimerization in living cells using bioluminescence resonance energy transfer (BRET). Proc Natl Acad Sci U S A 97:3684-9.

28. Antoine, G., F. Scheiflinger, F. Dorner, and F. G. Falkner. 1998. The complete genomic sequence of the modified vaccinia Ankara strain: comparison with other orthopoxviruses. Virology 244:365-96.

29. Appay, V., and S. L. Rowland-Jones. 2001. RANTES: a versatile and controversial chemokine. Trends Immunol 22:83-7. 
30. Appleyard, G., A. J. Hapel, and E. A. Boulter. 1971. An antigenic difference between intracellular and extracellular rabbitpox virus. J Gen Virol 13:9-17.

31. Arakawa, Y., J. V. Cordeiro, S. Schleich, T. P. Newsome, and M. Way. 2007. The release of vaccinia virus from infected cells requires RhoA-mDia modulation of cortical actin. Cell Host Microbe 1:227-40.

32. Arakawa, Y., J. V. Cordeiro, and M. Way. 2007. F11L-mediated inhibition of RhoA-mDia signaling stimulates microtubule dynamics during vaccinia virus infection. Cell Host Microbe 1:213-26.

33. Aramori, I., S. S. Ferguson, P. D. Bieniasz, J. Zhang, B. Cullen, and M. G. Cullen. 1997. Molecular mechanism of desensitization of the chemokine receptor CCR-5: receptor signaling and internalization are dissociable from its role as an HIV-1 co-receptor. EMBO J 16:4606-16.

34. Armstrong, J. A., D. H. Metz, and M. R. Young. 1973. The mode of entry of vaccinia virus into L cells. J Gen Virol 21:533-7.

35. Aspenstrom, P., U. Lindberg, and A. Hall. 1996. Two GTPases, Cdc42 and Rac, bind directly to a protein implicated in the immunodeficiency disorder Wiskott-Aldrich syndrome. Curr Biol 6:70-5.

36. Assarsson, E., J. A. Greenbaum, M. Sundstrom, L. Schaffer, J. A. Hammond, V. Pasquetto, C. Oseroff, R. C. Hendrickson, E. J. Lefkowitz, D. C. Tscharke, J. Sidney, H. M. Grey, S. R. Head, B. Peters, and A. Sette. 2008. Kinetic analysis of a complete poxvirus transcriptome reveals an immediateearly class of genes. Proc Natl Acad Sci U S A 105:2140-5.

37. Azzi, M., P. G. Charest, S. Angers, G. Rousseau, T. Kohout, M. Bouvier, and G. Pineyro. 2003. Beta-arrestin-mediated activation of MAPK by inverse agonists reveals distinct active conformations for G protein-coupled receptors. Proc Natl Acad Sci U S A 100:11406-11.

38. Baba, M., O. Nishimura, N. Kanzaki, M. Okamoto, H. Sawada, Y. Iizawa, M. Shiraishi, Y. Aramaki, K. Okonogi, Y. Ogawa, K. Meguro, and M. Fujino. 1999. A small-molecule, nonpeptide CCR5 antagonist with highly potent and selective anti-HIV-1 activity. Proc Natl Acad Sci U S A 96:5698-703.

39. Babcock, G. J., M. Farzan, and J. Sodroski. 2003. Ligand-independent dimerization of CXCR4, a principal HIV-1 coreceptor. J Biol Chem 278:337885.

40. Bacon, K. B., B. A. Premack, P. Gardner, and T. J. Schall. 1995. Activation of dual $\mathrm{T}$ cell signaling pathways by the chemokine RANTES. Science 269:172730.

41. Baek, S. H., J. Y. Kwak, S. H. Lee, T. Lee, S. H. Ryu, D. J. Uhlinger, and J. D. Lambeth. 1997. Lipase activities of p37, the major envelope protein of vaccinia virus. J Biol Chem 272:32042-9.

42. Baggiolini, M. 1998. Chemokines and leukocyte traffic. Nature 392:565-8.

43. Baggiolini, M. 2001. Chemokines in pathology and medicine. J Intern Med 250:91-104.

44. Baggiolini, M., and P. Loetscher. 2000. Chemokines in inflammation and immunity. Immunol Today 21:418-20. 
45. Baird, A. M., R. M. Gerstein, and L. J. Berg. 1999. The role of cytokine receptor signaling in lymphocyte development. Curr Opin Immunol 11:15766.

46. Bajetto, A., R. Bonavia, S. Barbero, and G. Schettini. 2002. Characterization of chemokines and their receptors in the central nervous system: physiopathological implications. J Neurochem 82:1311-29.

47. Balashov, K. E., J. B. Rottman, H. L. Weiner, and W. W. Hancock. 1999. CCR5(+) and CXCR3(+) T cells are increased in multiple sclerosis and their ligands MIP-1alpha and IP-10 are expressed in demyelinating brain lesions. Proc Natl Acad Sci U S A 96:6873-8.

48. Baldick, C. J., Jr., and B. Moss. 1993. Characterization and temporal regulation of mRNAs encoded by vaccinia virus intermediate-stage genes. J Virol 67:3515-27.

49. Baldwin, E. T., I. T. Weber, R. St Charles, J. C. Xuan, E. Appella, M. Yamada, K. Matsushima, B. F. Edwards, G. M. Clore, A. M. Gronenborn, and et al. 1991. Crystal structure of interleukin 8: symbiosis of NMR and crystallography. Proc Natl Acad Sci U S A 88:502-6.

50. Balkwill, F. 2004. Cancer and the chemokine network. Nat Rev Cancer 4:540-50.

51. Balkwill, F. 2003. Chemokine biology in cancer. Semin Immunol 15:49-55.

52. Balkwill, F. 2004. The significance of cancer cell expression of the chemokine receptor CXCR4. Semin Cancer Biol 14:171-9.

53. Banfield, B. W., Y. Leduc, L. Esford, K. Schubert, and F. Tufaro. 1995. Sequential isolation of proteoglycan synthesis mutants by using herpes simplex virus as a selective agent: evidence for a proteoglycan-independent virus entry pathway. J Virol 69:3290-8.

54. Baranowski, E., C. M. Ruiz-Jarabo, and E. Domingo. 2001. Evolution of cell recognition by viruses. Science 292:1102-5.

55. Barber, G. N. 2001. Host defense, viruses and apoptosis. Cell Death Differ 8:113-26.

56. Bardi, G., M. Lipp, M. Baggiolini, and P. Loetscher. 2001. The T cell chemokine receptor CCR7 is internalized on stimulation with ELC, but not with SLC. Eur J Immunol 31:3291-7.

57. Barnes, D. A., J. Tse, M. Kaufhold, M. Owen, J. Hesselgesser, R. Strieter, R. Horuk, and H. D. Perez. 1998. Polyclonal antibody directed against human RANTES ameliorates disease in the Lewis rat adjuvant-induced arthritis model. J Clin Invest 101:2910-9.

58. Barrett, J. W., J. X. Cao, S. Hota-Mitchell, and G. McFadden. 2001. Immunomodulatory proteins of myxoma virus. Semin Immunol 13:73-84.

59. Barry, M., S. F. Lee, L. Boshkov, and G. McFadden. 1995. Myxoma virus induces extensive CD4 downregulation and dissociation of p56lck in infected rabbit CD4+ T lymphocytes. J Virol 69:5243-51.

60. Barry, M., and G. McFadden. 1997. Virus encoded cytokines and cytokine receptors. Parasitology 115 Suppl:S89-100. 
61. Bartlett, N. W., K. Buttigieg, S. V. Kotenko, and G. L. Smith. 2005. Murine interferon lambdas (type III interferons) exhibit potent antiviral activity in vivo in a poxvirus infection model. J Gen Virol 86:1589-96.

62. Beattie, E., K. L. Denzler, J. Tartaglia, M. E. Perkus, E. Paoletti, and B. L. Jacobs. 1995. Reversal of the interferon-sensitive phenotype of a vaccinia virus lacking E3L by expression of the reovirus S4 gene. J Virol 69:499-505.

63. Beattie, E., E. B. Kauffman, H. Martinez, M. E. Perkus, B. L. Jacobs, E. Paoletti, and J. Tartaglia. 1996. Host-range restriction of vaccinia virus E3Lspecific deletion mutants. Virus Genes 12:89-94.

64. Behbehani, A. M. 1983. The smallpox story: life and death of an old disease. Microbiol Rev 47:455-509.

65. Belyakov, I. M., P. Earl, A. Dzutsev, V. A. Kuznetsov, M. Lemon, L. S. Wyatt, J. T. Snyder, J. D. Ahlers, G. Franchini, B. Moss, and J. A. Berzofsky. 2003. Shared modes of protection against poxvirus infection by attenuated and conventional smallpox vaccine viruses. Proc Natl Acad Sci U S A 100:945863.

66. Benjamin, C. W., D. A. Linseman, and D. A. Jones. 1994. Platelet-derived growth factor stimulates phosphorylation of growth factor receptor-binding protein-2 in vascular smooth muscle cells. J Biol Chem 269:31346-9.

67. Benkirane, M., D. Y. Jin, R. F. Chun, R. A. Koup, and K. T. Jeang. 1997. Mechanism of transdominant inhibition of CCR5-mediated HIV-1 infection by ccr5delta32. J Biol Chem 272:30603-6.

68. Bernfield, M., M. Gotte, P. W. Park, O. Reizes, M. L. Fitzgerald, J. Lincecum, and M. Zako. 1999. Functions of cell surface heparan sulfate proteoglycans. Annu Rev Biochem 68:729-77.

69. Bisht, H., A. S. Weisberg, and B. Moss. 2008. Vaccinia virus 11 protein is required for cell entry and membrane fusion. J Virol 82:8687-94.

70. Blanpain, C., B. J. Doranz, A. Bondue, C. Govaerts, A. De Leener, G. Vassart, R. W. Doms, A. Proudfoot, and M. Parmentier. 2003. The core domain of chemokines binds CCR5 extracellular domains while their amino terminus interacts with the transmembrane helix bundle. J Biol Chem 278:5179-87.

71. Blanpain, C., B. Lee, J. Vakili, B. J. Doranz, C. Govaerts, I. Migeotte, M. Sharron, V. Dupriez, G. Vassart, R. W. Doms, and M. Parmentier. 1999. Extracellular cysteines of CCR5 are required for chemokine binding, but dispensable for HIV-1 coreceptor activity. J Biol Chem 274:18902-8.

72. Blanpain, C., I. Migeotte, B. Lee, J. Vakili, B. J. Doranz, C. Govaerts, G. Vassart, R. W. Doms, and M. Parmentier. 1999. CCR5 binds multiple CCchemokines: MCP-3 acts as a natural antagonist. Blood 94:1899-905.

73. Blasco, R., and B. Moss. 1991. Extracellular vaccinia virus formation and cell-to-cell virus transmission are prevented by deletion of the gene encoding the 37,000-Dalton outer envelope protein. J Virol 65:5910-20.

74. Blasco, R., and B. Moss. 1992. Role of cell-associated enveloped vaccinia virus in cell-to-cell spread. J Virol 66:4170-9. 
75. Blasco, R., J. R. Sisler, and B. Moss. 1993. Dissociation of progeny vaccinia virus from the cell membrane is regulated by a viral envelope glycoprotein: effect of a point mutation in the lectin homology domain of the A34R gene. J Virol 67:3319-25.

76. Blattner, R. J., J. O. Norman, F. M. Heys, and I. Aksu. 1964. Antibody Response to Cutaneous Inoculation with Vaccinia Virus: Viremia and Viruria in Vaccinated Children. J Pediatr 64:839-52.

77. Boring, L., J. Gosling, F. S. Monteclaro, A. J. Lusis, C. L. Tsou, and I. F. Charo. 1996. Molecular cloning and functional expression of murine JE (monocyte chemoattractant protein 1) and murine macrophage inflammatory protein 1alpha receptors: evidence for two closely linked C-C chemokine receptors on chromosome 9. J Biol Chem 271:7551-8.

78. Born, T. L., L. A. Morrison, D. J. Esteban, T. VandenBos, L. G. Thebeau, N. Chen, M. K. Spriggs, J. E. Sims, and R. M. Buller. 2000. A poxvirus protein that binds to and inactivates IL-18, and inhibits NK cell response. J Immunol 164:3246-54.

79. Boshkov, L. K., J. L. Macen, and G. McFadden. 1992. Virus-induced loss of class I MHC antigens from the surface of cells infected with myxoma virus and malignant rabbit fibroma virus. J Immunol 148:881-7.

80. Boudreau, N., C. J. Sympson, Z. Werb, and M. J. Bissell. 1995. Suppression of ICE and apoptosis in mammary epithelial cells by extracellular matrix. Science 267:891-3.

81. Boulter, E. A. 1969. Protection against poxviruses. Proc R Soc Med 62:295-7.

82. Bowie, A., E. Kiss-Toth, J. A. Symons, G. L. Smith, S. K. Dower, and L. A. O'Neill. 2000. A46R and A52R from vaccinia virus are antagonists of host IL1 and toll-like receptor signaling. Proc Natl Acad Sci U S A 97:10162-7.

83. Brady, G., L. Boggan, A. Bowie, and L. A. O'Neill. 2005. Schlafen-1 causes a cell cycle arrest by inhibiting induction of cyclin D1. J Biol Chem 280:3072334.

84. Brandt, T. A., and B. L. Jacobs. 2001. Both carboxy- and amino-terminal domains of the vaccinia virus interferon resistance gene, E3L, are required for pathogenesis in a mouse model. J Virol 75:850-6.

85. Breman, J. G., and D. A. Henderson. 2002. Diagnosis and management of smallpox. N Engl J Med 346:1300-8.

86. Breman, J. G., and D. A. Henderson. 1998. Poxvirus dilemmas--monkeypox, smallpox, and biologic terrorism. N Engl J Med 339:556-9.

87. Brown, E., T. G. Senkevich, and B. Moss. 2006. Vaccinia virus F9 virion membrane protein is required for entry but not virus assembly, in contrast to the related L1 protein. J Virol 80:9455-64.

88. Broyles, S. S. 2003. Vaccinia virus transcription. J Gen Virol 84:2293-303.

89. Brum, L. M., P. C. Turner, H. Devick, M. T. Baquero, and R. W. Moyer. 2003. Plasma membrane localization and fusion inhibitory activity of the cowpox virus serpin SPI-3 require a functional signal sequence and the virus encoded hemagglutinin. Virology 306:289-302. 
90. Brutkiewicz, R. R., S. J. Klaus, and R. M. Welsh. 1992. Window of vulnerability of vaccinia virus-infected cells to natural killer (NK) cellmediated cytolysis correlates with enhanced NK cell triggering and is concomitant with a decrease in $\mathrm{H}-2$ class I antigen expression. Nat Immun 11:203-14.

91. Bukowski, J. F., B. A. Woda, S. Habu, K. Okumura, and R. M. Welsh. 1983. Natural killer cell depletion enhances virus synthesis and virus-induced hepatitis in vivo. J Immunol 131:1531-8.

92. Buller, R. M., J. Burnett, W. Chen, and J. Kreider. 1995. Replication of molluscum contagiosum virus. Virology 213:655-9.

93. Buller, R. M., and G. J. Palumbo. 1991. Poxvirus pathogenesis. Microbiol Rev 55:80-122.

94. Buller, R. M., M. Potter, and G. D. Wallace. 1986. Variable resistance to ectromelia (mousepox) virus among genera of Mus. Curr Top Microbiol Immunol 127:319-22.

95. Bundgaard, J. R., J. Vuust, and J. F. Rehfeld. 1997. New consensus features for tyrosine O-sulfation determined by mutational analysis. J Biol Chem 272:21700-5.

96. Bunemann, M., M. Frank, and M. J. Lohse. 2003. Gi protein activation in intact cells involves subunit rearrangement rather than dissociation. Proc Natl Acad Sci U S A 100:16077-82.

97. Burns, J. M., D. J. Dairaghi, M. Deitz, M. Tsang, and T. J. Schall. 2002. Comprehensive mapping of poxvirus vCCI chemokine-binding protein. Expanded range of ligand interactions and unusual dissociation kinetics. J Biol Chem 277:2785-9.

98. Bushman, F. D. 2002. Integration site selection by lentiviruses: biology and possible control. Curr Top Microbiol Immunol 261:165-77.

99. Butcher, E. C., and L. J. Picker. 1996. Lymphocyte homing and homeostasis. Science 272:60-6.

100. Byrne, J. A., M. Soloski, and J. A. Holowczak. 1983. Immune responses of DBA/2 mice bearing melanoma tumors: cell-mediated immune responses after challenge with vaccinia virus. Cancer Immunol Immunother 16:81-7.

101. Cabrera-Vera, T. M., J. Vanhauwe, T. O. Thomas, M. Medkova, A. Preininger, M. R. Mazzoni, and H. E. Hamm. 2003. Insights into G protein structure, function, and regulation. Endocr Rev 24:765-81.

102. Cameron, C., S. Hota-Mitchell, L. Chen, J. Barrett, J. X. Cao, C. Macaulay, D. Willer, D. Evans, and G. McFadden. 1999. The complete DNA sequence of myxoma virus. Virology 264:298-318.

103. Campellone, K. G., and J. M. Leong. 2005. Nck-independent actin assembly is mediated by two phosphorylated tyrosines within enteropathogenic Escherichia coli Tir. Mol Microbiol 56:416-32.

104. Cannon, K. S., D. N. Hebert, and A. Helenius. 1996. Glycan-dependent and independent association of vesicular stomatitis virus $\mathrm{G}$ protein with calnexin. J Biol Chem 271:14280-4. 
105. Cantrell, D. A. 2003. GTPases and T cell activation. Immunol Rev 192:12230.

106. Cao, J. X., P. D. Gershon, and D. N. Black. 1995. Sequence analysis of HindIII Q2 fragment of capripoxvirus reveals a putative gene encoding a G-proteincoupled chemokine receptor homologue. Virology 209:207-12.

107. Carlier, M. F., P. Nioche, I. Broutin-L'Hermite, R. Boujemaa, C. Le Clainche, C. Egile, C. Garbay, A. Ducruix, P. Sansonetti, and D. Pantaloni. 2000. GRB2 links signaling to actin assembly by enhancing interaction of neural Wiskott-Aldrich syndrome protein (N-WASp) with actin-related protein (ARP2/3) complex. J Biol Chem 275:21946-52.

108. Carman, C. V., and J. L. Benovic. 1998. G-protein-coupled receptors: turnons and turn-offs. Curr Opin Neurobiol 8:335-44.

109. Caron, E. 2002. Regulation of Wiskott-Aldrich syndrome protein and related molecules. Curr Opin Cell Biol 14:82-7.

110. Carrington, M., T. Kissner, B. Gerrard, S. Ivanov, S. J. O'Brien, and M. Dean. 1997. Novel alleles of the chemokine-receptor gene CCR5. Am J Hum Genet 61:1261-7.

111. Carter, G. C., M. Law, M. Hollinshead, and G. L. Smith. 2005. Entry of the vaccinia virus intracellular mature virion and its interactions with glycosaminoglycans. J Gen Virol 86:1279-90.

112. Carter, G. C., G. Rodger, B. J. Murphy, M. Law, O. Krauss, M. Hollinshead, and G. L. Smith. 2003. Vaccinia virus cores are transported on microtubules. J Gen Virol 84:2443-58.

113. Carter, N. M., S. Ali, and J. A. Kirby. 2003. Endothelial inflammation: the role of differential expression of $\mathrm{N}$-deacetylase/ $\mathrm{N}$-sulphotransferase enzymes in alteration of the immunological properties of heparan sulphate. J Cell Sci 116:3591-600.

114. Chahroudi, A., R. Chavan, N. Koyzr, E. K. Waller, G. Silvestri, and M. B. Feinberg. 2005. Vaccinia virus tropism for primary hematolymphoid cells is determined by restricted expression of a unique virus receptor. J Virol 79:10397-407.

115. Chahroudi, A., R. Chavan, N. Kozyr, E. K. Waller, G. Silvestri, and M. B. Feinberg. 2005. Vaccinia virus tropism for primary hematolymphoid cells is determined by restricted expression of a unique virus receptor. J Virol 79:10397-407.

116. Chambraud, B., C. Radanyi, J. H. Camonis, K. Shazand, K. Rajkowski, and E. E. Baulieu. 1996. FAP48, a new protein that forms specific complexes with both immunophilins FKBP59 and FKBP12. Prevention by the immunosuppressant drugs FK506 and rapamycin. J Biol Chem 271:32923-9.

117. Chang, A., and D. H. Metz. 1976. Further investigations on the mode of entry of vaccinia virus into cells. J Gen Virol 32:275-82.

118. Chang, H. W., J. C. Watson, and B. L. Jacobs. 1992. The E3L gene of vaccinia virus encodes an inhibitor of the interferon-induced, double-stranded RNAdependent protein kinase. Proc Natl Acad Sci U S A 89:4825-9. 
119. Chapes, S. K., and W. A. Tompkins. 1979. Cytotoxic macrophages induced in hamsters by vaccinia virus: selective cytotoxicity for virus-infected targets by macrophages collected late after immunization. J Immunol 123:303-10.

120. Charo, I. F., and M. B. Taubman. 2004. Chemokines in the pathogenesis of vascular disease. Circ Res 95:858-66.

121. Chen, Y. G., F. Liu, and J. Massague. 1997. Mechanism of TGFbeta receptor inhibition by FKBP12. Embo J 16:3866-76.

122. Child, S. J., C. A. Franke, and D. E. Hruby. 1988. Inhibition of vaccinia virus replication by nicotinamide: evidence for ADP-ribosylation of viral proteins. Virus Res 9:119-32.

123. Chiu, W. L., C. L. Lin, M. H. Yang, D. L. Tzou, and W. Chang. 2007. Vaccinia virus 4c (A26L) protein on intracellular mature virus binds to the extracellular cellular matrix laminin. J Virol 81:2149-57.

124. Chung, C. S., C. H. Chen, M. Y. Ho, C. Y. Huang, C. L. Liao, and W. Chang. 2006. Vaccinia virus proteome: identification of proteins in vaccinia virus intracellular mature virion particles. J Virol 80:2127-40.

125. Chung, C. S., J. C. Hsiao, Y. S. Chang, and W. Chang. 1998. A27L protein mediates vaccinia virus interaction with cell surface heparan sulfate. J Virol 72:1577-85.

126. Chung, C. S., C. Y. Huang, and W. Chang. 2005. Vaccinia virus penetration requires cholesterol and results in specific viral envelope proteins associated with lipid rafts. J Virol 79:1623-34.

127. Clark, C., P. G. McIntyre, A. Evans, C. J. McInnes, and S. Lewis-Jones. 2005. Human sealpox resulting from a seal bite: confirmation that sealpox virus is zoonotic. Br J Dermatol 152:791-3.

128. Clark-Lewis, I., B. Moser, A. Walz, M. Baggiolini, G. J. Scott, and R. Aebersold. 1991. Chemical synthesis, purification, and characterization of two inflammatory proteins, neutrophil activating peptide 1 (interleukin-8) and neutrophil activating peptide. Biochemistry 30:3128-35.

129. Clore, G. M., A. Bax, P. C. Driscoll, P. T. Wingfield, and A. M. Gronenborn. 1990. Assignment of the side-chain $1 \mathrm{H}$ and 13C resonances of interleukin-1 beta using double- and triple-resonance heteronuclear three-dimensional NMR spectroscopy. Biochemistry 29:8172-84.

130. Clore, G. M., and A. M. Gronenborn. 1995. Three-dimensional structures of alpha and beta chemokines. FASEB J 9:57-62.

131. Cocchi, F., A. L. DeVico, R. Yarchoan, R. Redfield, F. Cleghorn, W. A. Blattner, A. Garzino-Demo, S. Colombini-Hatch, D. Margolis, and R. C. Gallo. 2000. Higher macrophage inflammatory protein (MIP)-1alpha and MIP-1beta levels from CD8+ T cells are associated with asymptomatic HIV-1 infection. Proc Natl Acad Sci U S A 97:13812-7.

132. Colamonici, O. R., P. Domanski, S. M. Sweitzer, A. Larner, and R. M. Buller. 1995. Vaccinia virus B18R gene encodes a type I interferon-binding protein that blocks interferon alpha transmembrane signaling. J Biol Chem 270:15974-8. 
133. Comeau, M. R., R. Johnson, R. F. DuBose, M. Petersen, P. Gearing, T. VandenBos, L. Park, T. Farrah, R. M. Buller, J. I. Cohen, L. D. Strockbine, C. Rauch, and M. K. Spriggs. 1998. A poxvirus-encoded semaphorin induces cytokine production from monocytes and binds to a novel cellular semaphorin receptor, VESPR. Immunity 8:473-82.

134. Comerford, I., and R. J. Nibbs. 2005. Post-translational control of chemokines: a role for decoy receptors? Immunol Lett 96:163-74.

135. Condit, R. C., N. Moussatche, and P. Traktman. 2006. In a nutshell: structure and assembly of the vaccinia virion. Adv Virus Res 66:31-124.

136. Condit, R. C., and E. G. Niles. 2002. Regulation of viral transcription elongation and termination during vaccinia virus infection. Biochim Biophys Acta 1577:325-36.

137. Connor, R. I., H. Mohri, Y. Cao, and D. D. Ho. 1993. Increased viral burden and cytopathicity correlate temporally with CD4+ T-lymphocyte decline and clinical progression in human immunodeficiency virus type 1-infected individuals. J Virol 67:1772-7.

138. Connor, R. I., W. A. Paxton, K. E. Sheridan, and R. A. Koup. 1996. Macrophages and CD4+ T lymphocytes from two multiply exposed, uninfected individuals resist infection with primary non-syncytium-inducing isolates of human immunodeficiency virus type 1. J Virol 70:8758-64.

139. Connor, R. I., K. E. Sheridan, D. Ceradini, S. Choe, and N. R. Landau. 1997. Change in coreceptor use correlates with disease progression in HIV-1-infected individuals. J Exp Med 185:621-8.

140. Cooper, M. D., H. P. Chae, J. T. Lowman, W. Krivit, and R. A. Good. 1968. Wiskott-Aldrich syndrome. An immunologic deficiency disease involving the afferent limb of immunity. Am J Med 44:499-513.

141. Cornea, A., J. A. Janovick, G. Maya-Nunez, and P. M. Conn. 2001. Gonadotropin-releasing hormone receptor microaggregation. Rate monitored by fluorescence resonance energy transfer. J Biol Chem 276:21538.

142. Coupar, B. E., M. E. Andrew, G. W. Both, and D. B. Boyle. 1986. Temporal regulation of influenza hemagglutinin expression in vaccinia virus recombinants and effects on the immune response. Eur J Immunol 16:147987.

143. Crotty, S., P. Felgner, H. Davies, J. Glidewell, L. Villarreal, and R. Ahmed. 2003. Cutting edge: long-term B cell memory in humans after smallpox vaccination. J Immunol 171:4969-73.

144. Crump, M. P., K. Rajarathnam, K. S. Kim, I. Clark-Lewis, and B. D. Sykes. 1998. Solution structure of eotaxin, a chemokine that selectively recruits eosinophils in allergic inflammation. J Biol Chem 273:22471-9.

145. Cudmore, S., R. Blasco, R. Vincentelli, M. Esteban, B. Sodeik, G. Griffiths, and J. Krijnse Locker. 1996. A vaccinia virus core protein, p39, is membrane associated. J Virol 70:6909-21.

146. Cudmore, S., P. Cossart, G. Griffiths, and M. Way. 1995. Actin-based motility of vaccinia virus. Nature 378:636-8. 
147. Cunnion, K. M. 1999. Tumor necrosis factor receptors encoded by poxviruses. Mol Genet Metab 67:278-82.

148. D. Reynolds , K. K., R. Molestina, R. Srisatajluk, JHJ. Butterfield. 1999. Heparin binding activity of vaccinia virus complement control protein confers additional properties of uptake by mast cells and attachment to endothelial cells. Oxford/IBN, New Delhi.

149. Dairaghi, D. J., R. A. Fan, B. E. McMaster, M. R. Hanley, and T. J. Schall. 1999. HHV8-encoded vMIP-I selectively engages chemokine receptor CCR8. Agonist and antagonist profiles of viral chemokines. J Biol Chem 274:2156974.

150. Dales, S., and R. Kajioka. 1964. The Cycle of Multiplication of Vaccinia Virus in Earle's Strain L Cells. I. Uptake and Penetration. Virology 24:278-94.

151. Damaso, C. R., J. J. Esposito, R. C. Condit, and N. Moussatche. 2000. An emergent poxvirus from humans and cattle in Rio de Janeiro State: Cantagalo virus may derive from Brazilian smallpox vaccine. Virology 277:439-49.

152. Damaso, C. R., and N. Moussatche. 1998. Inhibition of vaccinia virus replication by cyclosporin A analogues correlates with their affinity for cellular cyclophilins. J Gen Virol 79 ( Pt 2):339-46.

153. Damon, I., P. M. Murphy, and B. Moss. 1998. Broad spectrum chemokine antagonistic activity of a human poxvirus chemokine homolog. Proc Natl Acad Sci U S A 95:6403-7.

154. Dao, T., W. Z. Mehal, and I. N. Crispe. 1998. IL-18 augments perforindependent cytotoxicity of liver NK-T cells. J Immunol 161:2217-22.

155. Dao, T., K. Ohashi, T. Kayano, M. Kurimoto, and H. Okamura. 1996. Interferon-gamma-inducing factor, a novel cytokine, enhances Fas ligandmediated cytotoxicity of murine T helper 1 cells. Cell Immunol 173:230-5.

156. Davies, D. H., D. M. Molina, J. Wrammert, J. Miller, S. Hirst, Y. Mu, J. Pablo, B. Unal, R. Nakajima-Sasaki, X. Liang, S. Crotty, K. L. Karem, I. K. Damon, R. Ahmed, L. Villarreal, and P. L. Felgner. 2007. Proteome-wide analysis of the serological response to vaccinia and smallpox. Proteomics 7:1678-86.

157. Davies, M. V., H. W. Chang, B. L. Jacobs, and R. J. Kaufman. 1993. The E3L and K3L vaccinia virus gene products stimulate translation through inhibition of the double-stranded RNA-dependent protein kinase by different mechanisms. J Virol 67:1688-92.

158. Dawson, T. C., M. A. Beck, W. A. Kuziel, F. Henderson, and N. Maeda. 2000. Contrasting effects of CCR5 and CCR2 deficiency in the pulmonary inflammatory response to influenza A virus. Am J Pathol 156:1951-9.

159. De Jong, J. J., A. De Ronde, W. Keulen, M. Tersmette, and J. Goudsmit. 1992. Minimal requirements for the human immunodeficiency virus type 1 V3 domain to support the syncytium-inducing phenotype: analysis by single amino acid substitution. J Virol 66:6777-80.

160. de Magalhaes, J. C., A. A. Andrade, P. N. Silva, L. P. Sousa, C. Ropert, P. C. Ferreira, E. G. Kroon, R. T. Gazzinelli, and C. A. Bonjardim. 2001. A mitogenic signal triggered at an early stage of vaccinia virus infection: 
implication of MEK/ERK and protein kinase A in virus multiplication. J Biol Chem 276:38353-60.

161. Dean, M., M. Carrington, C. Winkler, G. A. Huttley, M. W. Smith, R. Allikmets, J. J. Goedert, S. P. Buchbinder, E. Vittinghoff, E. Gomperts, S. Donfield, D. Vlahov, R. Kaslow, A. Saah, C. Rinaldo, R. Detels, and S. J. O'Brien. 1996. Genetic restriction of HIV-1 infection and progression to AIDS by a deletion allele of the CKR5 structural gene. Hemophilia Growth and Development Study, Multicenter AIDS Cohort Study, Multicenter Hemophilia Cohort Study, San Francisco City Cohort, ALIVE Study. Science 273:1856-62.

162. DeBruyne, L. A., K. Li, D. K. Bishop, and J. S. Bromberg. 2000. Gene transfer of virally encoded chemokine antagonists vMIP-II and MC148 prolongs cardiac allograft survival and inhibits donor-specific immunity. Gene Ther 7:575-82.

163. Devalaraja, M. N., and A. Richmond. 1999. Multiple chemotactic factors: fine control or redundancy? Trends Pharmacol Sci 20:151-6.

164. Devi, L. A. 2001. Heterodimerization of G-protein-coupled receptors: pharmacology, signaling and trafficking. Trends Pharmacol Sci 22:532-7.

165. Diven, D. G. 2001. An overview of poxviruses. J Am Acad Dermatol 44:1-16.

166. Dixon, C. W. 1963. Immunization against Smallpox. Br J Clin Pract 17:641-8.

167. Dobbelstein, M., and T. Shenk. 1996. Protection against apoptosis by the vaccinia virus SPI-2 (B13R) gene product. J Virol 70:6479-85.

168. Domanico, S. Z., and S. K. Pierce. 1992. Virus infection blocks the processing and presentation of exogenous antigen with the major histocompatibility complex class II molecules. Eur J Immunol 22:2055-62.

169. Domi, A., A. S. Weisberg, and B. Moss. 2008. Vaccinia virus E2L null mutants exhibit a major reduction in extracellular virion formation and virus spread. J Virol 82:4215-26.

170. Doms, R. W., R. Blumenthal, and B. Moss. 1990. Fusion of intra- and extracellular forms of vaccinia virus with the cell membrane. J Virol 64:488492.

171. Dragic, T., V. Litwin, G. P. Allaway, S. R. Martin, Y. Huang, K. A. Nagashima, C. Cayanan, P. J. Maddon, R. A. Koup, J. P. Moore, and W. A. Paxton. 1996. HIV-1 entry into CD4+ cells is mediated by the chemokine receptor CC-CKR-5. Nature 381:667-73.

172. Dragic, T., A. Trkola, D. A. Thompson, E. G. Cormier, F. A. Kajumo, E. Maxwell, S. W. Lin, W. Ying, S. O. Smith, T. P. Sakmar, and J. P. Moore. 2000. A binding pocket for a small molecule inhibitor of HIV-1 entry within the transmembrane helices of CCR5. Proc Natl Acad Sci U S A 97:5639-44.

173. Drillien, R., F. Koehren, and A. Kirn. 1981. Host range deletion mutant of vaccinia virus defective in human cells. Virology 111:488-99.

174. Drillien, R., D. Spehner, and A. Kirn. 1978. Host range restriction of vaccinia virus in Chinese hamster ovary cells: relationship to shutoff of protein synthesis. J Virol 28:843-50. 
175. Duncan, S. A., and G. L. Smith. 1992. Identification and characterization of an extracellular envelope glycoprotein affecting vaccinia virus egress. J Virol 66:1610-21.

176. Earley, A. K., W. M. Chan, and B. M. Ward. 2008. The vaccinia virus B5 protein requires A34 for efficient intracellular trafficking from the endoplasmic reticulum to the site of wrapping and incorporation into progeny virions. J Virol 82:2161-9.

177. Eaves, G., and T. H. Flewett. 1954. The structure of vaccinia virus. J Pathol Bacteriol 68:633-4.

178. Eckert, D. M., and P. S. Kim. 2001. Mechanisms of viral membrane fusion and its inhibition. Annu Rev Biochem 70:777-810.

179. Edghill-Smith, Y., H. Golding, J. Manischewitz, L. R. King, D. Scott, M. Bray, A. Nalca, J. W. Hooper, C. A. Whitehouse, J. E. Schmitz, K. A. Reimann, and G. Franchini. 2005. Smallpox vaccine-induced antibodies are necessary and sufficient for protection against monkeypox virus. Nat Med 11:740-7.

180. El-Asmar, L., J. Y. Springael, S. Ballet, E. U. Andrieu, G. Vassart, and M. Parmentier. 2005. Evidence for negative binding cooperativity within CCR5CCR2b heterodimers. Mol Pharmacol 67:460-9.

181. Engelmayer, J., M. Larsson, M. Subklewe, A. Chahroudi, W. I. Cox, R. M. Steinman, and N. Bhardwaj. 1999. Vaccinia virus inhibits the maturation of human dendritic cells: a novel mechanism of immune evasion. J Immunol 163:6762-8.

182. Engelstad, M., S. T. Howard, and G. L. Smith. 1992. A constitutively expressed vaccinia gene encodes a 42-kDa glycoprotein related to complement control factors that forms part of the extracellular virus envelope. Virology 188:801-10.

183. Engelstad, M., and G. L. Smith. 1993. The vaccinia virus 42-kDa envelope protein is required for the envelopment and egress of extracellular virus and for virus virulence. Virology 194:627-37.

184. Enoch, T., and P. Nurse. 1991. Coupling M phase and S phase: controls maintaining the dependence of mitosis on chromosome replication. Cell 65:921-3.

185. Eskra, L., A. Mathison, and G. Splitter. 2003. Microarray analysis of mRNA levels from RAW264.7 macrophages infected with Brucella abortus. Infect Immun 71:1125-33.

186. Esposito, J. J., S. A. Sammons, A. M. Frace, J. D. Osborne, M. OlsenRasmussen, M. Zhang, D. Govil, I. K. Damon, R. Kline, M. Laker, Y. Li, G. L. Smith, H. Meyer, J. W. Leduc, and R. M. Wohlhueter. 2006. Genome sequence diversity and clues to the evolution of variola (smallpox) virus. Science 313:807-12.

187. Farzan, M., T. Mirzabekov, P. Kolchinsky, R. Wyatt, M. Cayabyab, N. P. Gerard, C. Gerard, J. Sodroski, and H. Choe. 1999. Tyrosine sulfation of the amino terminus of CCR5 facilitates HIV-1 entry. Cell 96:667-76. 
188. Feng, Y., C. C. Broder, P. E. Kennedy, and E. A. Berger. 1996. HIV-1 entry cofactor: functional cDNA cloning of a seven-transmembrane, G proteincoupled receptor. Science 272:872-7.

189. Fenner, F. 1989. Risks and benefits of vaccinia vaccine use in the worldwide smallpox eradication campaign. Res Virol 140:465-6; discussion 487-91.

190. Fenner, F. 1993. Smallpox: emergence, global spread, and eradication. Hist Philos Life Sci 15:397-420.

191. Fenner, F. 1992. Vaccinia virus as a vaccine, and pox-virus pathogenesis . CRC Press, New York.

192. Fenner, F., and J. F. Sambrook. 1966. Conditional lethal mutants of rabbitpox virus. II. Mutants (p) that fail to multiply in PK-2a cells. Virology 28:600-9.

193. Fenner F., A. D. A., Arita I., Jezek Z., Ladnyi I.D., . 1988. Smallpox and Its Eradication. World Health Organisation, Geneva.

194. Fenner, F. H., DA; Arita, I; Jezek, Z; Ladnyi, ID. 1988. Smallpox and Its Eradication. WHO, Geneva.

195. Ferguson, S. S. 2001. Evolving concepts in G protein-coupled receptor endocytosis: the role in receptor desensitization and signaling. Pharmacol Rev 53:1-24.

196. Ferguson, S. S., J. Zhang, L. S. Barak, and M. G. Caron. 1998. Molecular mechanisms of $\mathrm{G}$ protein-coupled receptor desensitization and resensitization. Life Sci 62:1561-5.

197. Fernandez, E. J., and E. Lolis. 2002. Structure, function, and inhibition of chemokines. Annu Rev Pharmacol Toxicol 42:469-99.

198. Filizola, M., and H. Weinstein. 2002. Structural models for dimerization of G-protein coupled receptors: the opioid receptor homodimers. Biopolymers 66:317-25.

199. Floyd, D. H., A. Geva, S. P. Bruinsma, M. C. Overton, K. J. Blumer, and T. J. Baranski. 2003. C5a receptor oligomerization. II. Fluorescence resonance energy transfer studies of a human $G$ protein-coupled receptor expressed in yeast. J Biol Chem 278:35354-61.

200. Fraile-Ramos, A., T. A. Kohout, M. Waldhoer, and M. Marsh. 2003. Endocytosis of the viral chemokine receptor US28 does not require betaarrestins but is dependent on the clathrin-mediated pathway. Traffic 4:24353.

201. Franke, C. A., P. L. Reynolds, and D. E. Hruby. 1989. Fatty acid acylation of vaccinia virus proteins. J Virol 63:4285-91.

202. Franke, C. A., E. M. Wilson, and D. E. Hruby. 1990. Use of a cell-free system to identify the vaccinia virus L1R gene product as the major late myristylated virion protein M25. J Virol 64:5988-96.

203. Freer, G., and S. Senesi. 1993. No recognition of MHC class II+ cells infected with a vaccinia virus encoding influenza type A nucleoprotein by class IIrestricted T cells. Immunol Lett 36:305-12. 
204. Frischknecht, F., S. Cudmore, V. Moreau, I. Reckmann, S. Rottger, and M. Way. 1999. Tyrosine phosphorylation is required for actin-based motility of vaccinia but not Listeria or Shigella. Curr Biol 9:89-92.

205. Frischknecht, F., V. Moreau, S. Rottger, S. Gonfloni, I. Reckmann, G. Superti-Furga, and M. Way. 1999. Actin-based motility of vaccinia virus mimics receptor tyrosine kinase signalling. Nature 401:926-9.

206. Fujinami, R. S., M. G. von Herrath, U. Christen, and J. L. Whitton. 2006. Molecular mimicry, bystander activation, or viral persistence: infections and autoimmune disease. Clin Microbiol Rev 19:80-94.

207. Fulginiti V, K. C., Hathaway W, Pearlman D, SIEVER, O.F. JUN., ELLER, J. J., JOYNER, J.J., SEN. \& ROBINSON, A. 1968. Progressive vaccinia in immunologically deficient individuals. Birth Defects Original Articles Series 4:129-45.

208. G. McFadden, K. G., A. Opgenorth 1994. Poxvirus growth factors. Landes, Austin, TX.

209. Galligan, C. L., T. T. Murooka, R. Rahbar, E. Baig, B. Majchrzak-Kita, and E. N. Fish. 2006. Interferons and viruses: signaling for supremacy. Immunol Res 35:27-40.

210. Ganju, R. K., P. Dutt, L. Wu, W. Newman, H. Avraham, S. Avraham, and J. E. Groopman. 1998. Beta-chemokine receptor CCR5 signals via the novel tyrosine kinase RAFTK. Blood 91:791-7.

211. Gao, J. L., and P. M. Murphy. 1995. Cloning and differential tissue-specific expression of three mouse beta chemokine receptor-like genes, including the gene for a functional macrophage inflammatory protein-1 alpha receptor. J Biol Chem 270:17494-501.

212. Gao, P., X. Y. Zhou, Y. Yashiro-Ohtani, Y. F. Yang, N. Sugimoto, S. Ono, T. Nakanishi, S. Obika, T. Imanishi, T. Egawa, T. Nagasawa, H. Fujiwara, and T. Hamaoka. 2003. The unique target specificity of a nonpeptide chemokine receptor antagonist: selective blockade of two Th1 chemokine receptors CCR5 and CXCR3. J Leukoc Biol 73:273-80.

213. Gardner, J. D., D. C. Tscharke, P. C. Reading, and G. L. Smith. 2001. Vaccinia virus semaphorin A39R is a 50-55 kDa secreted glycoprotein that affects the outcome of infection in a murine intradermal model. J Gen Virol 82:2083-93.

214. Geada, M. M., I. Galindo, M. M. Lorenzo, B. Perdiguero, and R. Blasco. 2001. Movements of vaccinia virus intracellular enveloped virions with GFP tagged to the F13L envelope protein. J Gen Virol 82:2747-60.

215. Gemmell, A., and F. Fenner. 1960. Genetic studies with mammalian poxviruses. III. White (u) mutants of rabbitpox virus. Virology 11:219-35.

216. Gerard, C., and B. J. Rollins. 2001. Chemokines and disease. Nat Immunol 2:108-15.

217. Geserick, P., F. Kaiser, U. Klemm, S. H. Kaufmann, and J. Zerrahn. 2004. Modulation of T cell development and activation by novel members of the Schlafen (slfn) gene family harbouring an RNA helicase-like motif. Int Immunol 16:1535-48. 
218. Gether, U., and B. K. Kobilka. 1998. G protein-coupled receptors. II. Mechanism of agonist activation. J Biol Chem 273:17979-82.

219. Gil, J., and M. Esteban. 2000. Induction of apoptosis by the dsRNAdependent protein kinase (PKR): mechanism of action. Apoptosis 5:107-14.

220. Gil, J., J. Rullas, J. Alcami, and M. Esteban. 2001. MC159L protein from the poxvirus molluscum contagiosum virus inhibits NF-kappaB activation and apoptosis induced by PKR. J Gen Virol 82:3027-34.

221. Gilat, D., R. Hershkoviz, Y. A. Mekori, I. Vlodavsky, and O. Lider. 1994. Regulation of adhesion of CD4+ T lymphocytes to intact or heparinasetreated subendothelial extracellular matrix by diffusible or anchored RANTES and MIP-1 beta. J Immunol 153:4899-906.

222. Girard, F., U. Strausfeld, A. Fernandez, and N. J. Lamb. 1991. Cyclin A is required for the onset of DNA replication in mammalian fibroblasts. Cell 67:1169-79.

223. Glabinski, A. R., B. Bielecki, and R. M. Ransohoff. 2003. Chemokine upregulation follows cytokine expression in chronic relapsing experimental autoimmune encephalomyelitis. Scand J Immunol 58:81-8.

224. Glabinski, A. R., S. O'Bryant, K. Selmaj, and R. M. Ransohoff. 2000. CXC chemokine receptors expression during chronic relapsing experimental autoimmune encephalomyelitis. Ann N Y Acad Sci 917:135-44.

225. Glabinski, A. R., and R. M. Ransohoff. 1999. Chemokines and chemokine receptors in CNS pathology. J Neurovirol 5:3-12.

226. Glabinski, A. R., and R. M. Ransohoff. 1999. Sentries at the gate: chemokines and the blood-brain barrier. J Neurovirol 5:623-34.

227. Glass, W. G., D. H. McDermott, J. K. Lim, S. Lekhong, S. F. Yu, W. A. Frank, J. Pape, R. C. Cheshier, and P. M. Murphy. 2006. CCR5 deficiency increases risk of symptomatic West Nile virus infection. J Exp Med 203:35-40.

228. Godessart, N., and S. L. Kunkel. 2001. Chemokines in autoimmune disease. Curr Opin Immunol 13:670-5.

229. Goebel, S. J., G. P. Johnson, M. E. Perkus, S. W. Davis, J. P. Winslow, and E. Paoletti. 1990. The complete DNA sequence of vaccinia virus. Virology 179:247-66, 517-63.

230. Gomez Yafal, A., G. Kaplan, V. R. Racaniello, and J. M. Hogle. 1993. Characterization of poliovirus conformational alteration mediated by soluble cell receptors. Virology 197:501-5.

231. Gong, S. C., C. F. Lai, and M. Esteban. 1990. Vaccinia virus induces cell fusion at acid $\mathrm{pH}$ and this activity is mediated by the $\mathrm{N}$-terminus of the $14-\mathrm{kDa}$ virus envelope protein. Virology 178:81-91.

232. Gong, W., O. M. Howard, J. A. Turpin, M. C. Grimm, H. Ueda, P. W. Gray, C. J. Raport, J. J. Oppenheim, and J. M. Wang. 1998. Monocyte chemotactic protein-2 activates CCR5 and blocks CD4/CCR5-mediated HIV-1 entry/replication. J Biol Chem 273:4289-92.

233. Good, R. A., W. D. Kelly, J. Rotstein, and R. L. Varco. 1962. Immunological deficiency diseases. Agammaglobulinemia, hypogammaglobulinemia, Hodgkin's disease and sarcoidosis. Prog Allergy 6:187-319. 
234. Gortzak-Uzan, L., A. Ignatchenko, A. I. Evangelou, M. Agochiya, K. A. Brown, P. St Onge, I. Kireeva, G. Schmitt-Ulms, T. J. Brown, J. Murphy, B. Rosen, P. Shaw, I. Jurisica, and T. Kislinger. 2008. A proteome resource of ovarian cancer ascites: integrated proteomic and bioinformatic analyses to identify putative biomarkers. J Proteome Res 7:339-51.

235. Gosling, J., D. J. Dairaghi, Y. Wang, M. Hanley, D. Talbot, Z. Miao, and T. J. Schall. 2000. Cutting edge: identification of a novel chemokine receptor that binds dendritic cell- and T cell-active chemokines including ELC, SLC, and TECK. J Immunol 164:2851-6.

236. Gosling, J., S. Slaymaker, L. Gu, S. Tseng, C. H. Zlot, S. G. Young, B. J. Rollins, and I. F. Charo. 1999. MCP-1 deficiency reduces susceptibility to atherosclerosis in mice that overexpress human apolipoprotein B. J Clin Invest 103:773-8.

237. Govaerts, C., C. Blanpain, X. Deupi, S. Ballet, J. A. Ballesteros, S. J. Wodak, G. Vassart, L. Pardo, and M. Parmentier. 2001. The TXP motif in the second transmembrane helix of CCR5. A structural determinant of chemokineinduced activation. J Biol Chem 276:13217-25.

238. Grabenstein, J. D., and W. Winkenwerder, Jr. 2003. US military smallpox vaccination program experience. JAMA 289:3278-82.

239. Graham, G. J., and C. S. McKimmie. 2006. Chemokine scavenging by D6: a movable feast? Trends Immunol 27:381-6.

240. Graham, K. A., A. S. Lalani, J. L. Macen, T. L. Ness, M. Barry, L. Y. Liu, A. Lucas, I. Clark-Lewis, R. W. Moyer, and G. McFadden. 1997. The T1/35kDa family of poxvirus-secreted proteins bind chemokines and modulate leukocyte influx into virus-infected tissues. Virology 229:12-24.

241. Greaves, D. R., W. Wang, D. J. Dairaghi, M. C. Dieu, B. Saint-Vis, K. FranzBacon, D. Rossi, C. Caux, T. McClanahan, S. Gordon, A. Zlotnik, and T. J. Schall. 1997. CCR6, a CC chemokine receptor that interacts with macrophage inflammatory protein 3alpha and is highly expressed in human dendritic cells. J Exp Med 186:837-44.

242. Griffiths, G., R. Wepf, T. Wendt, J. K. Locker, M. Cyrklaff, and N. Roos. 2001. Structure and assembly of intracellular mature vaccinia virus: isolatedparticle analysis. J Virol 75:11034-55.

243. Grisendi, S., B. Chambraud, I. Gout, P. M. Comoglio, and T. Crepaldi. 2001. Ligand-regulated binding of FAP68 to the hepatocyte growth factor receptor. J Biol Chem 276:46632-8.

244. Grivel, J. C., M. L. Penn, D. A. Eckstein, B. Schramm, R. F. Speck, N. W. Abbey, B. Herndier, L. Margolis, and M. A. Goldsmith. 2000. Human immunodeficiency virus type 1 coreceptor preferences determine target Tcell depletion and cellular tropism in human lymphoid tissue. J Virol 74:5347-51.

245. Grosenbach, D. W., D. O. Ulaeto, and D. E. Hruby. 1997. Palmitylation of the vaccinia virus 37-kDa major envelope antigen. Identification of a conserved acceptor motif and biological relevance. J Biol Chem 272:1956-64. 
246. Gruenheid, S., L. Gatzke, H. Meadows, and F. Tufaro. 1993. Herpes simplex virus infection and propagation in a mouse $\mathrm{L}$ cell mutant lacking heparan sulfate proteoglycans. J Virol 67:93-100.

247. Gubser, C., R. Goodbody, A. Ecker, G. Brady, L. A. O'Neill, N. Jacobs, and G. L. Smith. 2007. Camelpox virus encodes a schlafen-like protein that affects orthopoxvirus virulence. J Gen Virol 88:1667-76.

248. Guerra, S., M. Aracil, R. Conde, A. Bernad, and M. Esteban. 2005. WiskottAldrich syndrome protein is needed for vaccinia virus pathogenesis. J Virol 79:2133-40.

249. Guerra, S., L. A. Lopez-Fernandez, R. Conde, A. Pascual-Montano, K. Harshman, and M. Esteban. 2004. Microarray analysis reveals characteristic changes of host cell gene expression in response to attenuated modified vaccinia virus Ankara infection of human HeLa cells. J Virol 78:5820-34.

250. Guerra, S., L. A. Lopez-Fernandez, A. Pascual-Montano, M. Munoz, K. Harshman, and M. Esteban. 2003. Cellular gene expression survey of vaccinia virus infection of human HeLa cells. J Virol 77:6493-506.

251. Gurevich, R. M., K. M. Regula, and L. A. Kirshenbaum. 2001. Serpin protein CrmA suppresses hypoxia-mediated apoptosis of ventricular myocytes. Circulation 103:1984-91.

252. Hamm, H. E. 1998. The many faces of G protein signaling. J Biol Chem 273:669-72.

253. Hammarlund, E., M. W. Lewis, S. G. Hansen, L. I. Strelow, J. A. Nelson, G. J. Sexton, J. M. Hanifin, and M. K. Slifka. 2003. Duration of antiviral immunity after smallpox vaccination. Nat Med 9:1131-7.

254. Hammond, S. M., Y. M. Altshuller, T. C. Sung, S. A. Rudge, K. Rose, J. Engebrecht, A. J. Morris, and M. A. Frohman. 1995. Human ADPribosylation factor-activated phosphatidylcholine-specific phospholipase D defines a new and highly conserved gene family. J Biol Chem 270:29640-3.

255. Handel, T. M., Z. Johnson, S. E. Crown, E. K. Lau, and A. E. Proudfoot. 2005. Regulation of protein function by glycosaminoglycans--as exemplified by chemokines. Annu Rev Biochem 74:385-410.

256. Harrington, L. E., R. Most Rv, J. L. Whitton, and R. Ahmed. 2002. Recombinant vaccinia virus-induced T-cell immunity: quantitation of the response to the virus vector and the foreign epitope. J Virol 76:3329-37.

257. Harrison, J. K., A. M. Fong, P. A. Swain, S. Chen, Y. R. Yu, M. N. Salafranca, W. B. Greenleaf, T. Imai, and D. D. Patel. 2001. Mutational analysis of the fractalkine chemokine domain. Basic amino acid residues differentially contribute to CX3CR1 binding, signaling, and cell adhesion. J Biol Chem 276:21632-41.

258. Harrop, R., M. G. Ryan, H. Golding, I. Redchenko, and M. W. Carroll. 2004. Monitoring of human immunological responses to vaccinia virus. Methods Mol Biol 269:243-66.

259. Hart, C. A., and N. J. Beeching. 2002. A spotlight on anthrax. Clin Dermatol 20:365-75. 
260. He, J., Y. Chen, M. Farzan, H. Choe, A. Ohagen, S. Gartner, J. Busciglio, X. Yang, W. Hofmann, W. Newman, C. R. Mackay, J. Sodroski, and D. Gabuzda. 1997. CCR3 and CCR5 are co-receptors for HIV-1 infection of microglia. Nature 385:645-9.

261. Hebert, C. A., R. V. Vitangcol, and J. B. Baker. 1991. Scanning mutagenesis of interleukin-8 identifies a cluster of residues required for receptor binding. J Biol Chem 266:18989-94.

262. Heinrich, P. C., I. Behrmann, S. Haan, H. M. Hermanns, G. Muller-Newen, and F. Schaper. 2003. Principles of interleukin (IL)-6-type cytokine signalling and its regulation. Biochem J 374:1-20.

263. Heldin, C. H. 1995. Dimerization of cell surface receptors in signal transduction. Cell 80:213-23.

264. Henderson, C. E., K. Bromek, N. P. Mullin, B. O. Smith, D. Uhrin, and P. N. Barlow. 2001. Solution structure and dynamics of the central CCP module pair of a poxvirus complement control protein. J Mol Biol 307:323-39.

265. Henderson, D., Moss B. 1999. Smallpox and vaccinia. WB Saunders, Philadelphia.

266. Henderson, D. A. 1999. Smallpox: clinical and epidemiologic features. Emerg Infect Dis 5:537-9.

267. Henderson, D. A., T. V. Inglesby, J. G. Bartlett, M. S. Ascher, E. Eitzen, P. B. Jahrling, J. Hauer, M. Layton, J. McDade, M. T. Osterholm, T. O'Toole, G. Parker, T. Perl, P. K. Russell, and K. Tonat. 1999. Smallpox as a biological weapon: medical and public health management. Working Group on Civilian Biodefense. Jama 281:2127-37.

268. Hernandez, L. D., L. R. Hoffman, T. G. Wolfsberg, and J. M. White. 1996. Virus-cell and cell-cell fusion. Annu Rev Cell Dev Biol 12:627-61.

269. Hernanz-Falcon, P., J. M. Rodriguez-Frade, A. Serrano, D. Juan, A. del Sol, S. F. Soriano, F. Roncal, L. Gomez, A. Valencia, A. C. Martinez, and M. Mellado. 2004. Identification of amino acid residues crucial for chemokine receptor dimerization. Nat Immunol 5:216-23.

270. Herold, S., W. von Wulffen, M. Steinmueller, S. Pleschka, W. A. Kuziel, M. Mack, M. Srivastava, W. Seeger, U. A. Maus, and J. Lohmeyer. 2006. Alveolar epithelial cells direct monocyte transepithelial migration upon influenza virus infection: impact of chemokines and adhesion molecules. J Immunol 177:1817-24.

271. Herrera, E., M. M. Lorenzo, R. Blasco, and S. N. Isaacs. 1998. Functional analysis of vaccinia virus B5R protein: essential role in virus envelopment is independent of a large portion of the extracellular domain. J Virol 72:294302.

272. Herrero-Martinez, E., K. L. Roberts, M. Hollinshead, and G. L. Smith. 2005. Vaccinia virus intracellular enveloped virions move to the cell periphery on microtubules in the absence of the A36R protein. J Gen Virol 86:2961-8. 
273. Hiller, G., and K. Weber. 1985. Golgi-derived membranes that contain an acylated viral polypeptide are used for vaccinia virus envelopment. J Virol 55:651-9.

274. Hirsch, E., V. L. Katanaev, C. Garlanda, O. Azzolino, L. Pirola, L. Silengo, S. Sozzani, A. Mantovani, F. Altruda, and M. P. Wymann. 2000. Central role for $\mathrm{G}$ protein-coupled phosphoinositide 3-kinase gamma in inflammation. Science 287:1049-53.

275. Hirt, P., G. Hiller, and R. Wittek. 1986. Localization and fine structure of a vaccinia virus gene encoding an envelope antigen. J Virol 58:757-64.

276. Hoffman, T. L., E. B. Stephens, O. Narayan, and R. W. Doms. 1998. HIV type I envelope determinants for use of the CCR2b, CCR3, STRL33, and APJ coreceptors. Proc Natl Acad Sci U S A 95:11360-5.

277. Hollinshead, M., G. Rodger, H. Van Eijl, M. Law, R. Hollinshead, D. J. Vaux, and G. L. Smith. 2001. Vaccinia virus utilizes microtubules for movement to the cell surface. J Cell Biol 154:389-402.

278. Hollinshead, M., A. Vanderplasschen, G. L. Smith, and D. J. Vaux. 1999. Vaccinia virus intracellular mature virions contain only one lipid membrane. J Virol 73:1503-17.

279. Homey, B., W. Wang, H. Soto, M. E. Buchanan, A. Wiesenborn, D. Catron, A. Muller, T. K. McClanahan, M. C. Dieu-Nosjean, R. Orozco, T. Ruzicka, P. Lehmann, E. Oldham, and A. Zlotnik. 2000. Cutting edge: the orphan chemokine receptor G protein-coupled receptor-2 (GPR-2, CCR10) binds the skin-associated chemokine CCL27 (CTACK/ALP/ILC). J Immunol 164:346570 .

280. Hopkins, R. J., and J. M. Lane. 2004. Clinical efficacy of intramuscular vaccinia immune globulin: a literature review. Clin Infect Dis 39:819-26.

281. Horuk, R. 2001. Chemokine receptors. Cytokine Growth Factor Rev 12:31335.

282. Horuk, R. 2003. Development and evaluation of pharmacological agents targeting chemokine receptors. Methods 29:369-75.

283. Hoshino, T., R. H. Wiltrout, and H. A. Young. 1999. IL-18 is a potent coinducer of IL-13 in NK and T cells: a new potential role for IL-18 in modulating the immune response. J Immunol 162:5070-7.

284. Hoshino, T., H. Yagita, J. R. Ortaldo, R. H. Wiltrout, and H. A. Young. 2000. In vivo administration of IL-18 can induce IgE production through Th2 cytokine induction and up-regulation of CD40 ligand (CD154) expression on CD4+ T cells. Eur J Immunol 30:1998-2006.

285. Howard, S. T., Y. S. Chan, and G. L. Smith. 1991. Vaccinia virus homologues of the Shope fibroma virus inverted terminal repeat proteins and a discontinuous ORF related to the tumor necrosis factor receptor family. Virology 180:633-47.

286. Hruby, D. E., D. L. Lynn, R. C. Condit, and J. R. Kates. 1980. Cellular differences in the molecular mechanisms of vaccinia virus host range restriction. J Gen Virol 47:485-8. 
287. Hruby, D. E., D. L. Lynn, and J. R. Kates. 1979. Vaccinia virus replication requires active participation of the host cell transcriptional apparatus. Proc Natl Acad Sci U S A 76:1887-90.

288. Hsiao, J. C., C. C. Chao, M. J. Young, Y. T. Chang, E. C. Cho, and W. Chang. 2006. A poxvirus host range protein, CP77, binds to a cellular protein, HMG20A, and regulates its dissociation from the vaccinia virus genome in CHO-K1 cells. J Virol 80:7714-28.

289. Hsiao, J. C., C. S. Chung, and W. Chang. 1998. Cell surface proteoglycans are necessary for A27L protein-mediated cell fusion: identification of the $\mathrm{N}$ terminal region of A27L protein as the glycosaminoglycan-binding domain. J Virol 72:8374-9.

290. Hsiao, J. C., C. S. Chung, and W. Chang. 1999. Vaccinia virus envelope D8L protein binds to cell surface chondroitin sulfate and mediates the adsorption of intracellular mature virions to cells. J Virol 73:8750-61.

291. Huang, C. Y., T. Y. Lu, C. H. Bair, Y. S. Chang, J. K. Jwo, and W. Chang. 2008. A novel cellular protein, VPEF, facilitates vaccinia virus penetration into HeLa cells through fluid phase endocytosis. J Virol 82:7988-99.

292. Huang, D., Y. Han, M. R. Rani, A. Glabinski, C. Trebst, T. Sorensen, M. Tani, J. Wang, P. Chien, S. O'Bryan, B. Bielecki, Z. L. Zhou, S. Majumder, and R. M. Ransohoff. 2000. Chemokines and chemokine receptors in inflammation of the nervous system: manifold roles and exquisite regulation. Immunol Rev 177:52-67.

293. Humlova, Z., M. Vokurka, M. Esteban, and Z. Melkova. 2002. Vaccinia virus induces apoptosis of infected macrophages. J Gen Virol 83:2821-32.

294. Husain, M., and B. Moss. 2002. Similarities in the induction of post-Golgi vesicles by the vaccinia virus F13L protein and phospholipase D. J Virol 76:7777-89.

295. Huttenrauch, F., A. Nitzki, F. T. Lin, S. Honing, and M. Oppermann. 2002. Beta-arrestin binding to $\mathrm{CC}$ chemokine receptor 5 requires multiple Cterminal receptor phosphorylation sites and involves a conserved Asp-ArgTyr sequence motif. J Biol Chem 277:30769-77.

296. Ichihashi, Y. 1996. Extracellular enveloped vaccinia virus escapes neutralization. Virology 217:478-85.

297. Ichihashi, Y., S. Matsumoto, and S. Dales. 1971. Biogenesis of poxviruses: role of A-type inclusions and host cell membranes in virus dissemination. Virology 46:507-32.

298. Ichihashi, Y., and M. Oie. 1996. Neutralizing epitope on penetration protein of vaccinia virus. Virology 220:491-4.

299. Ichihashi, Y., M. Oie, and T. Tsuruhara. 1984. Location of DNA-binding proteins and disulfide-linked proteins in vaccinia virus structural elements. J Virol 50:929-38.

300. Isaacs, S. N., E. J. Wolffe, L. G. Payne, and B. Moss. 1992. Characterization of a vaccinia virus-encoded 42 -kilodalton class I membrane glycoprotein component of the extracellular virus envelope. J Virol 66:7217-24. 
301. Isaguliants, M. G. 2003. Hepatitis C virus clearance: the enigma of failure despite an impeccable survival strategy. Curr Pharm Biotechnol 4:169-83.

302. Isaguliants, M. G., and N. N. Ozeretskovskaya. 2003. Host background factors contributing to hepatitis $\mathrm{C}$ virus clearance. Curr Pharm Biotechnol 4:185-93.

303. Ishikawa-Mochizuki, I., M. Kitaura, M. Baba, T. Nakayama, D. Izawa, T. Imai, H. Yamada, K. Hieshima, R. Suzuki, H. Nomiyama, and O. Yoshie. 1999. Molecular cloning of a novel CC chemokine, interleukin-11 receptor alpha-locus chemokine (ILC), which is located on chromosome 9p13 and a potential homologue of a CC chemokine encoded by molluscum contagiosum virus. FEBS Lett 460:544-8.

304. Issafras, H., S. Angers, S. Bulenger, C. Blanpain, M. Parmentier, C. LabbeJullie, M. Bouvier, and S. Marullo. 2002. Constitutive agonist-independent CCR5 oligomerization and antibody-mediated clustering occurring at physiological levels of receptors. J Biol Chem 277:34666-73.

305. Iwasaki, M., T. Mukai, P. Gao, W. R. Park, C. Nakajima, M. Tomura, H. Fujiwara, and T. Hamaoka. 2001. A critical role for IL-12 in CCR5 induction on T cell receptor-triggered mouse CD4(+) and CD8(+) T cells. Eur J Immunol 31:2411-20.

306. Jacobs, B. L., and J. O. Langland. 1996. When two strands are better than one: the mediators and modulators of the cellular responses to doublestranded RNA. Virology 219:339-49.

307. Jacobs, N., R. A. Chen, C. Gubser, P. Najarro, and G. L. Smith. 2006. Intradermal immune response after infection with Vaccinia virus. J Gen Virol 87:1157-61.

308. Janeczko, R. A., J. F. Rodriguez, and M. Esteban. 1987. Studies on the mechanism of entry of vaccinia virus in animal cells. Arch Virol 92:135-50.

309. Jekle, A., O. T. Keppler, E. De Clercq, D. Schols, M. Weinstein, and M. A. Goldsmith. 2003. In vivo evolution of human immunodeficiency virus type 1 toward increased pathogenicity through CXCR4-mediated killing of uninfected CD4 T cells. J Virol 77:5846-54.

310. Jekle, A., B. Schramm, P. Jayakumar, V. Trautner, D. Schols, E. De Clercq, J. Mills, S. M. Crowe, and M. A. Goldsmith. 2002. Coreceptor phenotype of natural human immunodeficiency virus with nef deleted evolves in vivo, leading to increased virulence. J Virol 76:6966-73.

311. Jensen, O. N., T. Houthaeve, A. Shevchenko, S. Cudmore, T. Ashford, M. Mann, G. Griffiths, and J. Krijnse Locker. 1996. Identification of the major membrane and core proteins of vaccinia virus by two-dimensional electrophoresis. J Virol 70:7485-97.

312. Johannes, L., and C. Lamaze. 2002. Clathrin-dependent or not: is it still the question? Traffic 3:443-51.

313. Johnson, Z., M. H. Kosco-Vilbois, S. Herren, R. Cirillo, V. Muzio, P. Zaratin, M. Carbonatto, M. Mack, A. Smailbegovic, M. Rose, R. Lever, C. Page, T. N. Wells, and A. E. Proudfoot. 2004. Interference with heparin binding and 
oligomerization creates a novel anti-inflammatory strategy targeting the chemokine system. J Immunol 173:5776-85.

314. Johnston, J. B., J. W. Barrett, W. Chang, C. S. Chung, W. Zeng, J. Masters, M. Mann, F. Wang, J. Cao, and G. McFadden. 2003. Role of the serine-threonine kinase PAK-1 in myxoma virus replication. J Virol 77:5877-88.

315. Johnston, J. B., and G. McFadden. 2003. Poxvirus immunomodulatory strategies: current perspectives. J Virol 77:6093-100.

316. Johnston, J. B., and G. McFadden. 2004. Technical knockout: understanding poxvirus pathogenesis by selectively deleting viral immunomodulatory genes. Cell Microbiol 6:695-705.

317. Kaplan, G., M. S. Freistadt, and V. R. Racaniello. 1990. Neutralization of poliovirus by cell receptors expressed in insect cells. J Virol 64:4697-702.

318. Karpus, W. J., N. W. Lukacs, B. L. McRae, R. M. Strieter, S. L. Kunkel, and S. D. Miller. 1995. An important role for the chemokine macrophage inflammatory protein- 1 alpha in the pathogenesis of the T cell-mediated autoimmune disease, experimental autoimmune encephalomyelitis. J Immunol 155:5003-10.

319. Katz, E., E. Wolffe, and B. Moss. 2002. Identification of second-site mutations that enhance release and spread of vaccinia virus. J Virol 76:11637-44.

320. Katze, M. G., Y. He, and M. Gale, Jr. 2002. Viruses and interferon: a fight for supremacy. Nat Rev Immunol 2:675-87.

321. Kaupmann, K., B. Malitschek, V. Schuler, J. Heid, W. Froestl, P. Beck, J. Mosbacher, S. Bischoff, A. Kulik, R. Shigemoto, A. Karschin, and B. Bettler. 1998. GABA(B)-receptor subtypes assemble into functional heteromeric complexes. Nature 396:683-7.

322. Kawagishi-Kobayashi, M., C. Cao, J. Lu, K. Ozato, and T. E. Dever. 2000. Pseudosubstrate inhibition of protein kinase PKR by swine pox virus C8L gene product. Virology 276:424-34.

323. Kennedy, K. J., R. M. Strieter, S. L. Kunkel, N. W. Lukacs, and W. J. Karpus. 1998. Acute and relapsing experimental autoimmune encephalomyelitis are regulated by differential expression of the CC chemokines macrophage inflammatory protein-1alpha and monocyte chemotactic protein-1. J Neuroimmunol 92:98-108.

324. Kermorgant, S., and P. J. Parker. 2005. c-Met signalling: spatio-temporal decisions. Cell Cycle 4:352-5.

325. Kermorgant, S., D. Zicha, and P. J. Parker. 2004. PKC controls HGFdependent c-Met traffic, signalling and cell migration. Embo J 23:3721-34.

326. Kettle, S., A. Alcami, A. Khanna, R. Ehret, C. Jassoy, and G. L. Smith. 1997. Vaccinia virus serpin B13R (SPI-2) inhibits interleukin-1beta-converting enzyme and protects virus-infected cells from TNF- and Fas-mediated apoptosis, but does not prevent IL-1beta-induced fever. J Gen Virol 78 ( Pt 3):677-85. 
327. Khoja, H., G. Wang, C. T. Ng, J. Tucker, T. Brown, and V. Shyamala. 2000. Cloning of CCRL1, an orphan seven transmembrane receptor related to chemokine receptors, expressed abundantly in the heart. Gene 246:229-38.

328. Kirkitadze, M. D., C. Henderson, N. C. Price, S. M. Kelly, N. P. Mullin, J. Parkinson, D. T. Dryden, and P. N. Barlow. 1999. Central modules of the vaccinia virus complement control protein are not in extensive contact. Biochem J 344 Pt 1:167-75.

329. Kislinger, T., B. Cox, A. Kannan, C. Chung, P. Hu, A. Ignatchenko, M. S. Scott, A. O. Gramolini, Q. Morris, M. T. Hallett, J. Rossant, T. R. Hughes, B. Frey, and A. Emili. 2006. Global survey of organ and organelle protein expression in mouse: combined proteomic and transcriptomic profiling. Cell 125:173-86.

330. Kisseleva, T., S. Bhattacharya, J. Braunstein, and C. W. Schindler. 2002. Signaling through the JAK/STAT pathway, recent advances and future challenges. Gene 285:1-24.

331. Kitaura, M., N. Suzuki, T. Imai, S. Takagi, R. Suzuki, T. Nakajima, K. Hirai, H. Nomiyama, and O. Yoshie. 1999. Molecular cloning of a novel human CC chemokine (Eotaxin-3) that is a functional ligand of CC chemokine receptor 3. J Biol Chem 274:27975-80.

332. Kjellen, L., and U. Lindahl. 1991. Proteoglycans: structures and interactions. Annu Rev Biochem 60:443-75.

333. Koch, A. E., S. L. Kunkel, L. A. Harlow, D. D. Mazarakis, G. K. Haines, M. D. Burdick, R. M. Pope, and R. M. Strieter. 1994. Macrophage inflammatory protein-1 alpha. A novel chemotactic cytokine for macrophages in rheumatoid arthritis. J Clin Invest 93:921-8.

334. Kohout, T. A., S. L. Nicholas, S. J. Perry, G. Reinhart, S. Junger, and R. S. Struthers. 2004. Differential desensitization, receptor phosphorylation, beta-arrestin recruitment, and ERK1/2 activation by the two endogenous ligands for the CC chemokine receptor 7. J Biol Chem 279:23214-22.

335. Kolluri, R., K. F. Tolias, C. L. Carpenter, F. S. Rosen, and T. Kirchhausen. 1996. Direct interaction of the Wiskott-Aldrich syndrome protein with the GTPase Cdc42. Proc Natl Acad Sci U S A 93:5615-8.

336. Kolodkin, A. L., D. J. Matthes, and C. S. Goodman. 1993. The semaphorin genes encode a family of transmembrane and secreted growth cone guidance molecules. Cell 75:1389-99.

337. Komano, J., K. Miyauchi, Z. Matsuda, and N. Yamamoto. 2004. Inhibiting the Arp2/3 complex limits infection of both intracellular mature vaccinia virus and primate lentiviruses. Mol Biol Cell 15:5197-207.

338. Koopmann, W., and M. S. Krangel. 1997. Identification of a glycosaminoglycan-binding site in chemokine macrophage inflammatory protein-1alpha. J Biol Chem 272:10103-9.

339. Kotwal, G. J., S. N. Isaacs, R. McKenzie, M. M. Frank, and B. Moss. 1990. Inhibition of the complement cascade by the major secretory protein of vaccinia virus. Science 250:827-30. 
340. Kotwal, G. J., and B. Moss. 1988. Analysis of a large cluster of nonessential genes deleted from a vaccinia virus terminal transposition mutant. Virology 167:524-37.

341. Kotwal, G. J., and B. Moss. 1988. Vaccinia virus encodes a secretory polypeptide structurally related to complement control proteins. Nature 335:176-8.

342. Kozinn, P. J., M. M. Sigel, and R. Gorrie. 1955. Progressive vaccinia associated with agammaglobulinemia and defects in immune mechanism. Pediatrics 16:600-8.

343. Kraft, K., H. Olbrich, I. Majoul, M. Mack, A. Proudfoot, and M. Oppermann. 2001. Characterization of sequence determinants within the carboxyl-terminal domain of chemokine receptor CCR5 that regulate signaling and receptor internalization. J Biol Chem 276:34408-18.

344. Kreisberg, J. F., D. Kwa, B. Schramm, V. Trautner, R. Connor, H. Schuitemaker, J. I. Mullins, A. B. van't Wout, and M. A. Goldsmith. 2001. Cytopathicity of human immunodeficiency virus type 1 primary isolates depends on coreceptor usage and not patient disease status. J Virol 75:88427.

345. Kugler, S., N. Klocker, P. Kermer, S. Isenmann, and M. Bahr. 1999. Transduction of axotomized retinal ganglion cells by adenoviral vector administration at the optic nerve stump: an in vivo model system for the inhibition of neuronal apoptotic cell death. Gene Ther 6:1759-67.

346. Kulbe, H., N. R. Levinson, F. Balkwill, and J. L. Wilson. 2004. The chemokine network in cancer--much more than directing cell movement. Int J Dev Biol 48:489-96.

347. Kunkel, S. L. 1999. Promiscuous chemokine receptors and their redundant ligands play an enigmatic role during HIV-1 infection. Am J Respir Cell Mol Biol 20:859-60.

348. Kunkel, S. L., and N. Godessart. 2002. Chemokines in autoimmunity: from pathology to therapeutics. Autoimmun Rev 1:313-20.

349. Kuschert, G. S., F. Coulin, C. A. Power, A. E. Proudfoot, R. E. Hubbard, A. J. Hoogewerf, and T. N. Wells. 1999. Glycosaminoglycans interact selectively with chemokines and modulate receptor binding and cellular responses. Biochemistry 38:12959-68.

350. Kuschert, G. S., A. J. Hoogewerf, A. E. Proudfoot, C. W. Chung, R. M. Cooke, R. E. Hubbard, T. N. Wells, and P. N. Sanderson. 1998. Identification of a glycosaminoglycan binding surface on human interleukin-8. Biochemistry 37:11193-201.

351. Kwak, H., H. Horig, and H. L. Kaufman. 2003. Poxviruses as vectors for cancer immunotherapy. Curr Opin Drug Discov Devel 6:161-8.

352. Lakadamyali, M., M. J. Rust, and X. Zhuang. 2006. Ligands for clathrinmediated endocytosis are differentially sorted into distinct populations of early endosomes. Cell 124:997-1009.

353. Lalani, A. S., J. W. Barrett, and G. McFadden. 2000. Modulating chemokines: more lessons from viruses. Immunol Today 21:100-6. 
354. Lalani, A. S., K. Graham, K. Mossman, K. Rajarathnam, I. Clark-Lewis, D. Kelvin, and G. McFadden. 1997. The purified myxoma virus gamma interferon receptor homolog M-T7 interacts with the heparin-binding domains of chemokines. J Virol 71:4356-63.

355. Lalani, A. S., J. Masters, W. Zeng, J. Barrett, R. Pannu, H. Everett, C. W. Arendt, and G. McFadden. 1999. Use of chemokine receptors by poxviruses. Science 286:1968-71.

356. Lalani, A. S., and G. McFadden. 1997. Secreted poxvirus chemokine binding proteins. J Leukoc Biol 62:570-6.

357. Lalani, A. S., T. L. Ness, R. Singh, J. K. Harrison, B. T. Seet, D. J. Kelvin, G. McFadden, and R. W. Moyer. 1998. Functional comparisons among members of the poxvirus T1/35kDa family of soluble CC-chemokine inhibitor glycoproteins. Virology 250:173-84.

358. Lalezari, J., M. Thompson, P. Kumar, P. Piliero, R. Davey, K. Patterson, A. Shachoy-Clark, K. Adkison, J. Demarest, Y. Lou, M. Berrey, and S. Piscitelli. 2005. Antiviral activity and safety of 873140 , a novel CCR5 antagonist, during short-term monotherapy in HIV-infected adults. AIDS 19:1443-8.

359. Lane, J. M., and J. D. Millar. 1971. Risks of smallpox vaccination complications in the United States. Am J Epidemiol 93:238-40.

360. Lane, J. M., F. L. Ruben, J. M. Neff, and J. D. Millar. 1969. Complications of smallpox vaccination, 1968. N Engl J Med 281:1201-8.

361. Lane, J. M., F. L. Ruben, J. M. Neff, and J. D. Millar. 1970. Complications of smallpox vaccination, 1968: results of ten statewide surveys. J Infect Dis 122:303-9.

362. LaRosa, G. J., K. M. Thomas, M. E. Kaufmann, R. Mark, M. White, L. Taylor, G. Gray, D. Witt, and J. Navarro. 1992. Amino terminus of the interleukin-8 receptor is a major determinant of receptor subtype specificity. J Biol Chem 267:25402-6.

363. Law, M., and G. L. Smith. 2004. Studying the binding and entry of the intracellular and extracellular enveloped forms of vaccinia virus. Methods Mol Biol 269:187-204.

364. Lee, D. M., and M. E. Weinblatt. 2001. Rheumatoid arthritis. Lancet 358:903-11.

365. Lee, H. J., K. Essani, and G. L. Smith. 2001. The genome sequence of Yabalike disease virus, a yatapoxvirus. Virology 281:170-92.

366. Lehner, P. J., J. T. Karttunen, G. W. Wilkinson, and P. Cresswell. 1997. The human cytomegalovirus US6 glycoprotein inhibits transporter associated with antigen processing-dependent peptide translocation. Proc Natl Acad Sci U S A 94:6904-9.

367. Lewis-Jones, S. 2002. The zoonotic poxviruses. Dermatol Nurs 14:79-82, 856; quiz 87.

368. Lewis-Jones, S. 2004. Zoonotic poxvirus infections in humans. Curr Opin Infect Dis 17:81-9. 
369. Li, S., A. D. Couvillon, B. B. Brasher, and R. A. Van Etten. 2001. Tyrosine phosphorylation of Grb2 by Bcr/Abl and epidermal growth factor receptor: a novel regulatory mechanism for tyrosine kinase signaling. EMBO J 20:6793804.

370. Li, Y., R. L. Hall, and R. W. Moyer. 1997. Transient, nonlethal expression of genes in vertebrate cells by recombinant entomopoxviruses. J Virol 71:955762.

371. Li, Z., H. Jiang, W. Xie, Z. Zhang, A. V. Smrcka, and D. Wu. 2000. Roles of PLC-beta2 and -beta3 and PI3Kgamma in chemoattractant-mediated signal transduction. Science 287:1046-9.

372. Lin, C. L., C. S. Chung, H. G. Heine, and W. Chang. 2000. Vaccinia virus envelope H3L protein binds to cell surface heparan sulfate and is important for intracellular mature virion morphogenesis and virus infection in vitro and in vivo. J Virol 74:3353-65.

373. Lin, F. T., Y. Daaka, and R. J. Lefkowitz. 1998. beta-arrestins regulate mitogenic signaling and clathrin-mediated endocytosis of the insulin-like growth factor I receptor. J Biol Chem 273:31640-3.

374. Lin, F. T., K. M. Krueger, H. E. Kendall, Y. Daaka, Z. L. Fredericks, J. A. Pitcher, and R. J. Lefkowitz. 1997. Clathrin-mediated endocytosis of the beta-adrenergic receptor is regulated by phosphorylation/dephosphorylation of beta-arrestin1. J Biol Chem 272:31051-7.

375. Lipsey, A. I., M. J. Kahn, and R. P. Bolande. 1967. Pathologic variants of congenital hypogamma-globulinemia: an analysis of 3 patients dying of measles. Pediatrics 39:659-74.

376. Liptakova, H., E. Kontsekova, A. Alcami, G. L. Smith, and P. Kontsek. 1997. Analysis of an interaction between the soluble vaccinia virus-coded type I interferon (IFN)-receptor and human IFN-alpha1 and IFN-alpha2. Virology 232:86-90.

377. Littman, D. R. 1998. Chemokine receptors: keys to AIDS pathogenesis? Cell 93:677-80.

378. Liu, L., Z. Xu, R. C. Fuhlbrigge, V. Pena-Cruz, J. Lieberman, and T. S. Kupper. 2005. Vaccinia virus induces strong immunoregulatory cytokine production in healthy human epidermal keratinocytes: a novel strategy for immune evasion. J Virol 79:7363-70.

379. Liu, R., W. A. Paxton, S. Choe, D. Ceradini, S. R. Martin, R. Horuk, M. E. MacDonald, H. Stuhlmann, R. A. Koup, and N. R. Landau. 1996. Homozygous defect in HIV-1 coreceptor accounts for resistance of some multiply-exposed individuals to HIV-1 infection. Cell 86:367-77.

380. Liu, S., S. Fan, and Z. Sun. 2003. Structural and functional characterization of the human CCR5 receptor in complex with HIV gp120 envelope glycoprotein and CD4 receptor by molecular modeling studies. J Mol Model 9:329-36.

381. Liu, Y., K. C. Wolff, B. L. Jacobs, and C. E. Samuel. 2001. Vaccinia virus E3L interferon resistance protein inhibits the interferon-induced adenosine deaminase A-to-I editing activity. Virology 289:378-87. 
382. Lloyd, C. M., A. W. Minto, M. E. Dorf, A. Proudfoot, T. N. Wells, D. J. Salant, and J. C. Gutierrez-Ramos. 1997. RANTES and monocyte chemoattractant protein-1 (MCP-1) play an important role in the inflammatory phase of crescentic nephritis, but only MCP-1 is involved in crescent formation and interstitial fibrosis. J Exp Med 185:1371-80.

383. Locati, M., and P. M. Murphy. 1999. Chemokines and chemokine receptors: biology and clinical relevance in inflammation and AIDS. Annu Rev Med 50:425-40.

384. Locker, J. K., and G. Griffiths. 1999. An unconventional role for cytoplasmic disulfide bonds in vaccinia virus proteins. J Cell Biol 144:267-79.

385. Locker, J. K., A. Kuehn, S. Schleich, G. Rutter, H. Hohenberg, R. Wepf, and G. Griffiths. 2000. Entry of the two infectious forms of vaccinia virus at the plasma membane is signaling-dependent for the IMV but not the EEV. Mol Biol Cell 11:2497-511.

386. Loetscher, P., and I. Clark-Lewis. 2001. Agonistic and antagonistic activities of chemokines. J Leukoc Biol 69:881-4.

387. Lopez-Ilasaca, M., P. Crespo, P. G. Pellici, J. S. Gutkind, and R. Wetzker. 1997. Linkage of G protein-coupled receptors to the MAPK signaling pathway through PI 3-kinase gamma. Science 275:394-7.

388. Lorenzo, M. M., E. Herrera, R. Blasco, and S. N. Isaacs. 1998. Functional analysis of vaccinia virus B5R protein: role of the cytoplasmic tail. Virology 252:450-7.

389. Lu, Z. H., Z. X. Wang, R. Horuk, J. Hesselgesser, Y. C. Lou, T. J. Hadley, and S. C. Peiper. 1995. The promiscuous chemokine binding profile of the Duffy antigen/receptor for chemokines is primarily localized to sequences in the amino-terminal domain. J Biol Chem 270:26239-45.

390. Lusso, P. 2006. HIV and the chemokine system: 10 years later. EMBO J 25:447-56.

391. Lusso, P., P. L. Earl, F. Sironi, F. Santoro, C. Ripamonti, G. Scarlatti, R. Longhi, E. A. Berger, and S. E. Burastero. 2005. Cryptic nature of a conserved, CD4-inducible V3 loop neutralization epitope in the native envelope glycoprotein oligomer of CCR5-restricted, but not CXCR4-using, primary human immunodeficiency virus type 1 strains. J Virol 79:6957-68.

392. Luster, A. D., S. M. Greenberg, and P. Leder. 1995. The IP-10 chemokine binds to a specific cell surface heparan sulfate site shared with platelet factor 4 and inhibits endothelial cell proliferation. J Exp Med 182:219-31.

393. Luster, A. D., S. C. Jhanwar, R. S. Chaganti, J. H. Kersey, and J. V. Ravetch. 1987. Interferon-inducible gene maps to a chromosomal band associated with a $(4 ; 11)$ translocation in acute leukemia cells. Proc Natl Acad Sci U S A 84:2868-71.

394. Luttichau, H. R., J. Gerstoft, and T. W. Schwartz. 2001. MC148 encoded by human molluscum contagiosum poxvirus is an antagonist for human but not murine CCR8. J Leukoc Biol 70:277-82.

395. Maa, J. S., and M. Esteban. 1987. Structural and functional studies of a 39,000-Mr immunodominant protein of vaccinia virus. J Virol 61:3910-9. 
396. Macaulay, T. B. 1849. The History of England from the Accession of James II by Thomas Macaulay, vol. 1. PHILADELPHIA:PORTER \& COATES, London.

397. Macen, J. L., K. A. Graham, S. F. Lee, M. Schreiber, L. K. Boshkov, and G. McFadden. 1996. Expression of the myxoma virus tumor necrosis factor receptor homologue and M11L genes is required to prevent virus-induced apoptosis in infected rabbit T lymphocytes. Virology 218:232-7.

398. Machesky, L. M., R. D. Mullins, H. N. Higgs, D. A. Kaiser, L. Blanchoin, R. C. May, M. E. Hall, and T. D. Pollard. 1999. Scar, a WASp-related protein, activates nucleation of actin filaments by the Arp2/3 complex. Proc Natl Acad Sci U S A 96:3739-44.

399. Mack, M., B. Luckow, P. J. Nelson, J. Cihak, G. Simmons, P. R. Clapham, N. Signoret, M. Marsh, M. Stangassinger, F. Borlat, T. N. Wells, D.

Schlondorff, and A. E. Proudfoot. 1998. Aminooxypentane-RANTES induces CCR5 internalization but inhibits recycling: a novel inhibitory mechanism of HIV infectivity. J Exp Med 187:1215-24.

400. Mackett, M., G. L. Smith, and B. Moss. 1992. Vaccinia virus: a selectable eukaryotic cloning and expression vector. 1982. Biotechnology 24:495-9.

401. Maddon, P. J., J. S. McDougal, P. R. Clapham, A. G. Dalgleish, S. Jamal, R. A. Weiss, and R. Axel. 1988. HIV infection does not require endocytosis of its receptor, CD4. Cell 54:865-74.

402. Maeda, H., and A. Shiraishi. 1996. TGF-beta contributes to the shift toward Th2-type responses through direct and IL-10-mediated pathways in tumorbearing mice. J Immunol 156:73-8.

403. Maeda, K., H. Nakata, Y. Koh, T. Miyakawa, H. Ogata, Y. Takaoka, S. Shibayama, K. Sagawa, D. Fukushima, J. Moravek, Y. Koyanagi, and H. Mitsuya. 2004. Spirodiketopiperazine-based CCR5 inhibitor which preserves CC-chemokine/CCR5 interactions and exerts potent activity against R5 human immunodeficiency virus type 1 in vitro. J Virol 78:8654-62.

404. Mammoto, A., S. Huang, K. Moore, P. Oh, and D. E. Ingber. 2004. Role of RhoA, mDia, and ROCK in cell shape-dependent control of the Skp2-p27kip1 pathway and the G1/S transition. J Biol Chem 279:26323-30.

405. Mantovani, A. 1999. The chemokine system: redundancy for robust outputs. Immunol Today 20:254-7.

406. Mantovani, A., M. Locati, A. Vecchi, S. Sozzani, and P. Allavena. 2001. Decoy receptors: a strategy to regulate inflammatory cytokines and chemokines. Trends Immunol 22:328-36.

407. Marchese, A., C. Chen, Y. M. Kim, and J. L. Benovic. 2003. The ins and outs of G protein-coupled receptor trafficking. Trends Biochem Sci 28:369-76.

408. Mariani, M., R. Lang, E. Binda, P. Panina-Bordignon, and D. D'Ambrosio. 2004. Dominance of CCL22 over CCL17 in induction of chemokine receptor CCR4 desensitization and internalization on human Th2 cells. Eur J Immunol 34:231-40.

409. Marsh, M., and A. Helenius. 1989. Virus entry into animal cells. Adv Virus Res 36:107-51. 
410. Martin, L., C. Blanpain, P. Garnier, V. Wittamer, M. Parmentier, and C. Vita. 2001. Structural and functional analysis of the RANTESglycosaminoglycans interactions. Biochemistry 40:6303-18.

411. Martinez-Pomares, L., R. J. Stern, and R. W. Moyer. 1993. The ps/hr gene (B5R open reading frame homolog) of rabbitpox virus controls pock color, is a component of extracellular enveloped virus, and is secreted into the medium. J Virol 67:5450-62.

412. Martinez-Pomares, L., J. P. Thompson, and R. W. Moyer. 1995. Mapping and investigation of the role in pathogenesis of the major unique secreted 35kDa protein of rabbitpox virus. Virology 206:591-600.

413. Massung, R. F., J. J. Esposito, L. I. Liu, J. Qi, T. R. Utterback, J. C. Knight, L. Aubin, T. E. Yuran, J. M. Parsons, V. N. Loparev, and et al. 1993. Potential virulence determinants in terminal regions of variola smallpox virus genome. Nature 366:748-51.

414. Massung, R. F., V. Jayarama, and R. W. Moyer. 1993. DNA sequence analysis of conserved and unique regions of swinepox virus: identification of genetic elements supporting phenotypic observations including a novel $\mathrm{G}$ protein-coupled receptor homologue. Virology 197:511-28.

415. Massung, R. F., L. I. Liu, J. Qi, J. C. Knight, T. E. Yuran, A. R. Kerlavage, J. M. Parsons, J. C. Venter, and J. J. Esposito. 1994. Analysis of the complete genome of smallpox variola major virus strain Bangladesh-1975. Virology 201:215-40.

416. Masters, J., A. A. Hinek, S. Uddin, L. C. Platanias, W. Zeng, G. McFadden, and E. N. Fish. 2001. Poxvirus infection rapidly activates tyrosine kinase signal transduction. J Biol Chem 276:48371-5.

417. Mathew, E., C. M. Sanderson, M. Hollinshead, and G. L. Smith. 1998. The extracellular domain of vaccinia virus protein B5R affects plaque phenotype, extracellular enveloped virus release, and intracellular actin tail formation. J Virol 72:2429-38.

418. Mathew, E. C., C. M. Sanderson, R. Hollinshead, and G. L. Smith. 2001. A mutational analysis of the vaccinia virus B5R protein. J Gen Virol 82:1199213.

419. Mayo, K. H., E. Ilyina, V. Roongta, M. Dundas, J. Joseph, C. K. Lai, T. Maione, and T. J. Daly. 1995. Heparin binding to platelet factor-4. An NMR and site-directed mutagenesis study: arginine residues are crucial for binding. Biochem J 312 ( Pt 2):357-65.

420. McCarty, J. H. 1998. The Nck SH2/SH3 adaptor protein: a regulator of multiple intracellular signal transduction events. Bioessays 20:913-21.

421. McClain, M. E. 1965. The Host Range and Plaque Morphology of Rabbitpox Virus (Rpu+) and Its U Mutants on Chick Fibroblast, Pk-2a, and L929 Cells. Aust J Exp Biol Med Sci 43:31-44.

422. McClure, M. O., M. Marsh, and R. A. Weiss. 1988. Human immunodeficiency virus infection of CD4-bearing cells occurs by a pH-independent mechanism. EMBO J 7:513-8.

423. McFadden, G. 2005. Poxvirus tropism. Nat Rev Microbiol 3:201-13. 
424. McFadden, G., and K. Kane. 1994. How DNA viruses perturb functional MHC expression to alter immune recognition. Adv Cancer Res 63:117-209.

425. McInnes, C. J., A. R. Wood, and A. A. Mercer. 1998. Orf virus encodes a homolog of the vaccinia virus interferon-resistance gene E3L. Virus Genes 17:107-15.

426. McInnes, C. J., A. R. Wood, P. E. Nettleton, and J. A. Gilray. 2001. Genomic comparison of an avirulent strain of Orf virus with that of a virulent wild type isolate reveals that the Orf virus G2L gene is non-essential for replication. Virus Genes 22:141-50.

427. McIntosh, A. A., and G. L. Smith. 1996. Vaccinia virus glycoprotein A34R is required for infectivity of extracellular enveloped virus. J Virol 70:272-81.

428. Melby, T., and M. Westby. 2009. Inhibitors of viral entry. Handb Exp Pharmacol:177-202.

429. Mellado, M., J. M. Rodriguez-Frade, S. Manes, and A. C. Martinez. 2001. Chemokine signaling and functional responses: the role of receptor dimerization and TK pathway activation. Annu Rev Immunol 19:397-421.

430. Mellado, M., J. M. Rodriguez-Frade, A. J. Vila-Coro, S. Fernandez, A. Martin de Ana, D. R. Jones, J. L. Toran, and A. C. Martinez. 2001. Chemokine receptor homo- or heterodimerization activates distinct signaling pathways. EMBO J 20:2497-507.

431. Mellado, M., A. J. Vila-Coro, C. Martinez, and J. M. Rodriguez-Frade. 2001. Receptor dimerization: a key step in chemokine signaling. Cell Mol Biol (Noisy-le-grand) 47:575-82.

432. Mendelsohn, C. L., E. Wimmer, and V. R. Racaniello. 1989. Cellular receptor for poliovirus: molecular cloning, nucleotide sequence, and expression of a new member of the immunoglobulin superfamily. Cell 56:855-65.

433. Mercer, J., and A. Helenius. 2008. Vaccinia virus uses macropinocytosis and apoptotic mimicry to enter host cells. Science 320:531-5.

434. Messud-Petit, F., J. Gelfi, M. Delverdier, M. F. Amardeilh, R. Py, G. Sutter, and S. Bertagnoli. 1998. Serp2, an inhibitor of the interleukin-1betaconverting enzyme, is critical in the pathobiology of myxoma virus. J Virol 72:7830-9.

435. Meydan, N., T. Grunberger, H. Dadi, M. Shahar, E. Arpaia, Z. Lapidot, J. S. Leeder, M. Freedman, A. Cohen, A. Gazit, A. Levitzki, and C. M. Roifman. 1996. Inhibition of acute lymphoblastic leukaemia by a Jak-2 inhibitor. Nature 379:645-8.

436. Micallef, M. J., T. Ohtsuki, K. Kohno, F. Tanabe, S. Ushio, M. Namba, T. Tanimoto, K. Torigoe, M. Fujii, M. Ikeda, S. Fukuda, and M. Kurimoto. 1996. Interferon-gamma-inducing factor enhances T helper 1 cytokine production by stimulated human T cells: synergism with interleukin-12 for interferon-gamma production. Eur J Immunol 26:1647-51.

437. Middleton, J., S. Neil, J. Wintle, I. Clark-Lewis, H. Moore, C. Lam, M. Auer, E. Hub, and A. Rot. 1997. Transcytosis and surface presentation of IL-8 by venular endothelial cells. Cell 91:385-95. 
438. Miki, H., K. Miura, and T. Takenawa. 1996. N-WASP, a novel actindepolymerizing protein, regulates the cortical cytoskeletal rearrangement in a PIP2-dependent manner downstream of tyrosine kinases. EMBO J 15:532635.

439. Milligan, G. 2002. Construction and analysis of function of G protein-coupled receptor-G protein fusion proteins. Methods Enzymol 343:260-73.

440. Milligan, G., and J. H. White. 2001. Protein-protein interactions at Gprotein-coupled receptors. Trends Pharmacol Sci 22:513-8.

441. Moench, S. J., J. Moreland, D. H. Stewart, and T. G. Dewey. 1994. Fluorescence studies of the location and membrane accessibility of the palmitoylation sites of rhodopsin. Biochemistry 33:5791-6.

442. Montgomery, R. I., M. S. Warner, B. J. Lum, and P. G. Spear. 1996. Herpes simplex virus-1 entry into cells mediated by a novel member of the TNF/NGF receptor family. Cell 87:427-36.

443. Morikawa, S., and Y. Ueda. 1993. Characterization of vaccinia surface antigen expressed by recombinant baculovirus. Virology 193:753-61.

444. Morrison, L. A., A. E. Lukacher, V. L. Braciale, D. P. Fan, and T. J. Braciale. 1986. Differences in antigen presentation to MHC class I-and class IIrestricted influenza virus-specific cytolytic T lymphocyte clones. J Exp Med 163:903-21.

445. Moser, B. 2003. Chemokines: role in immune cell traffic. Eur Cytokine Netw 14:204-10.

446. Moser, B., M. Wolf, A. Walz, and P. Loetscher. 2004. Chemokines: multiple levels of leukocyte migration control. Trends Immunol 25:75-84.

447. Moss, B. 2001. Fields Virology, 4th. ed. Lippincott williams \& wilkins, Philadelpia.

448. Moss, B. 1996. Poxviridae : the viruses and their replication, 3rd. ed. Lippincott-Raven, Philadelphia.

449. Moss, B. 2001. Poxviridae: the viruses and their replication, vol. vol. 2. Lippincott-Raven Publishers, Philadelphia.

450. Moss, B. 2007. Poxviridae: The Viruses and Their Replication. Lippincott Williams \& Wilkins, Phila.

451. Moss, B. 2001. Poxviridae: the viruses and their replication, vol. 2. Lippincott-Raven Publishers, Philadelphia.

452. Moss, B. 2006. Poxvirus entry and membrane fusion. Virology 344:48-54.

453. Moss, B. 1985. Replication of poxviruses. Raven Press, New York.

454. Moss, B., M. W. Carroll, L. S. Wyatt, J. R. Bennink, V. M. Hirsch, S. Goldstein, W. R. Elkins, T. R. Fuerst, J. D. Lifson, M. Piatak, N. P. Restifo, W. Overwijk, R. Chamberlain, S. A. Rosenberg, and G. Sutter. 1996. Host range restricted, non-replicating vaccinia virus vectors as vaccine candidates. Adv Exp Med Biol 397:7-13.

455. Moss, B., and B. M. Ward. 2001. High-speed mass transit for poxviruses on microtubules. Nat Cell Biol 3:E245-6. 
456. Mossman, K., C. Upton, R. M. Buller, and G. McFadden. 1995. Species specificity of ectromelia virus and vaccinia virus interferon-gamma binding proteins. Virology 208:762-9.

457. Mueller, A., E. Kelly, and P. G. Strange. 2002. Pathways for internalization and recycling of the chemokine receptor CCR5. Blood 99:785-91.

458. Mueller, A., N. G. Mahmoud, M. C. Goedecke, J. A. McKeating, and P. G. Strange. 2002. Pharmacological characterization of the chemokine receptor, CCR5. Br J Pharmacol 135:1033-43.

459. Mueller, A., and P. G. Strange. 2004. Mechanisms of internalization and recycling of the chemokine receptor, CCR5. Eur J Biochem 271:243-52.

460. Mukaida, N., M. Shiroo, and K. Matsushima. 1989. Genomic structure of the human monocyte-derived neutrophil chemotactic factor IL-8. J Immunol 143:1366-71.

461. Muller, D. M., M. P. Pender, and J. M. Greer. 2004. Chemokines and chemokine receptors: potential therapeutic targets in multiple sclerosis. Curr Drug Targets Inflamm Allergy 3:279-90.

462. Mullins, R. D., J. A. Heuser, and T. D. Pollard. 1998. The interaction of Arp2/3 complex with actin: nucleation, high affinity pointed end capping, and formation of branching networks of filaments. Proc Natl Acad Sci U S A 95:6181-6.

463. Murdoch, C., P. N. Monk, and A. Finn. 1999. Cxc chemokine receptor expression on human endothelial cells. Cytokine 11:704-12.

464. Murooka, T. T., R. Rahbar, L. C. Platanias, and E. N. Fish. 2008. CCL5mediated T-cell chemotaxis involves the initiation of mRNA translation through mTOR/4E-BP1. Blood 111:4892-901.

465. Murooka, T. T., M. M. Wong, R. Rahbar, B. Majchrzak-Kita, A. E. Proudfoot, and E. N. Fish. 2006. CCL5-CCR5-mediated apoptosis in T cells: Requirement for glycosaminoglycan binding and CCL5 aggregation. J Biol Chem 281:25184-94.

466. Murphy, P. M. 2001. Viral exploitation and subversion of the immune system through chemokine mimicry. Nat Immunol 2:116-22.

467. Murphy, P. M., M. Baggiolini, I. F. Charo, C. A. Hebert, R. Horuk, K. Matsushima, L. H. Miller, J. J. Oppenheim, and C. A. Power. 2000. International union of pharmacology. XXII. Nomenclature for chemokine receptors. Pharmacol Rev 52:145-76.

468. Murthy, K. H., S. A. Smith, V. K. Ganesh, K. W. Judge, N. Mullin, P. N. Barlow, C. M. Ogata, and G. J. Kotwal. 2001. Crystal structure of a complement control protein that regulates both pathways of complement activation and binds heparan sulfate proteoglycans. Cell 104:301-11.

469. Najarro, P., P. Traktman, and J. A. Lewis. 2001. Vaccinia virus blocks gamma interferon signal transduction: viral VH1 phosphatase reverses Stat1 activation. J Virol 75:3185-96.

470. Nanki, T., K. Nagasaka, K. Hayashida, Y. Saita, and N. Miyasaka. 2001. Chemokines regulate IL-6 and IL-8 production by fibroblast-like 
synoviocytes from patients with rheumatoid arthritis. J Immunol 167:53815.

471. Napier, K. B., Z. X. Wang, S. C. Peiper, and J. O. Trent. 2007. CCR5 interactions with the variable 3 loop of gp120. J Mol Model 13:29-41.

472. Nash, P., J. Barrett, J. X. Cao, S. Hota-Mitchell, A. S. Lalani, H. Everett, X. M. Xu, J. Robichaud, S. Hnatiuk, C. Ainslie, B. T. Seet, and G. McFadden. 1999. Immunomodulation by viruses: the myxoma virus story. Immunol Rev 168:103-20.

473. Neff, J. M., J. M. Lane, J. H. Pert, R. Moore, J. D. Millar, and D. A.

Henderson. 1967. Complications of smallpox vaccination. I. National survey in the United States, 1963. N Engl J Med 276:125-32.

474. Neves, S. R., P. T. Ram, and R. Iyengar. 2002. G protein pathways. Science 296:1636-9.

475. Newsome, T. P., N. Scaplehorn, and M. Way. 2004. SRC mediates a switch from microtubule- to actin-based motility of vaccinia virus. Science 306:1249.

476. Ng, H. P., K. May, J. G. Bauman, A. Ghannam, I. Islam, M. Liang, R. Horuk, J. Hesselgesser, R. M. Snider, H. D. Perez, and M. M. Morrissey. 1999. Discovery of novel non-peptide CCR1 receptor antagonists. J Med Chem 42:4680-94.

477. Nibbs, R. J., S. M. Wylie, I. B. Pragnell, and G. J. Graham. 1997. Cloning and characterization of a novel murine beta chemokine receptor, D6. Comparison to three other related macrophage inflammatory protein-1alpha receptors, CCR-1, CCR-3, and CCR-5. J Biol Chem 272:12495-504.

478. Nibbs, R. J., S. M. Wylie, J. Yang, N. R. Landau, and G. J. Graham. 1997. Cloning and characterization of a novel promiscuous human beta-chemokine receptor D6. J Biol Chem 272:32078-83.

479. Nichols, B. 2003. Caveosomes and endocytosis of lipid rafts. J Cell Sci 116:4707-14.

480. Nichols, B. J., and J. Lippincott-Schwartz. 2001. Endocytosis without clathrin coats. Trends Cell Biol 11:406-12.

481. Nichols, R. J., E. Stanitsa, B. Unger, and P. Traktman. 2008. The vaccinia virus gene I2L encodes a membrane protein with an essential role in virion entry. J Virol 82:10247-61.

482. Nielsen, M., K. Kaltoft, M. Nordahl, C. Ropke, C. Geisler, T. Mustelin, P. Dobson, A. Svejgaard, and N. Odum. 1997. Constitutive activation of a slowly migrating isoform of Stat3 in mycosis fungoides: tyrphostin AG490 inhibits Stat3 activation and growth of mycosis fungoides tumor cell lines. Proc Natl Acad Sci U S A 94:6764-9.

483. Nobes, C. D., P. Hawkins, L. Stephens, and A. Hall. 1995. Activation of the small GTP-binding proteins rho and rac by growth factor receptors. J Cell Sci 108 ( Pt 1):225-33.

484. Novick, D., S. H. Kim, G. Fantuzzi, L. L. Reznikov, C. A. Dinarello, and M. Rubinstein. 1999. Interleukin-18 binding protein: a novel modulator of the Th1 cytokine response. Immunity 10:127-36. 
485. O'Connell, C. J., D. T. Karzon, A. L. Barron, M. E. Plaut, and V. M. Ali. 1964. Progressive Vaccinia with Normal Antibodies. A Case Possibly Due to Deficient Cellular Immunity. Ann Intern Med 60:282-9.

486. O'Shea, J. J., M. Gadina, and R. D. Schreiber. 2002. Cytokine signaling in 2002: new surprises in the Jak/Stat pathway. Cell 109 Suppl:S121-31.

487. Ogilvie, P., G. Bardi, I. Clark-Lewis, M. Baggiolini, and M. Uguccioni. 2001. Eotaxin is a natural antagonist for CCR2 and an agonist for CCR5. Blood 97:1920-4.

488. Ogilvie, P., S. Paoletti, I. Clark-Lewis, and M. Uguccioni. 2003. Eotaxin-3 is a natural antagonist for CCR2 and exerts a repulsive effect on human monocytes. Blood 102:789-94.

489. Oie, K. L., and D. J. Pickup. 2001. Cowpox virus and other members of the orthopoxvirus genus interfere with the regulation of NF-kappaB activation. Virology 288:175-87.

490. Ojeda, S., T. G. Senkevich, and B. Moss. 2006. Entry of vaccinia virus and cell-cell fusion require a highly conserved cysteine-rich membrane protein encoded by the A16L gene. J Virol 80:51-61.

491. Okamoto, T., A. Schlegel, P. E. Scherer, and M. P. Lisanti. 1998. Caveolins, a family of scaffolding proteins for organizing "preassembled signaling complexes" at the plasma membrane. J Biol Chem 273:5419-22.

492. Okamura, H., H. Tsutsi, T. Komatsu, M. Yutsudo, A. Hakura, T. Tanimoto, K. Torigoe, T. Okura, Y. Nukada, K. Hattori, and et al. 1995. Cloning of a new cytokine that induces IFN-gamma production by T cells. Nature 378:8891.

493. Onuffer, J. J., and R. Horuk. 2002. Chemokines, chemokine receptors and small-molecule antagonists: recent developments. Trends Pharmacol Sci 23:459-67.

494. Oppenheim, J. J., C. O. Zachariae, N. Mukaida, and K. Matsushima. 1991. Properties of the novel proinflammatory supergene "intercrine" cytokine family. Annu Rev Immunol 9:617-48.

495. Oppermann, M. 2004. Chemokine receptor CCR5: insights into structure, function, and regulation. Cell Signal 16:1201-10.

496. Oppermann, M., M. Mack, A. E. Proudfoot, and H. Olbrich. 1999. Differential effects of CC chemokines on CC chemokine receptor 5 (CCR5) phosphorylation and identification of phosphorylation sites on the CCR5 carboxyl terminus. J Biol Chem 274:8875-85.

497. Orlandi, P. A., and P. H. Fishman. 1998. Filipin-dependent inhibition of cholera toxin: evidence for toxin internalization and activation through caveolae-like domains. J Cell Biol 141:905-15.

498. Overton, M. C., and K. J. Blumer. 2002. The extracellular N-terminal domain and transmembrane domains 1 and 2 mediate oligomerization of a yeast $\mathrm{G}$ protein-coupled receptor. J Biol Chem 277:41463-72.

499. Pagano, M., R. Pepperkok, F. Verde, W. Ansorge, and G. Draetta. 1992. Cyclin A is required at two points in the human cell cycle. EMBO J 11:961-71. 
500. Pamer, E., and P. Cresswell. 1998. Mechanisms of MHC class I--restricted antigen processing. Annu Rev Immunol 16:323-58.

501. Panicali, D., and E. Paoletti. 1992. Construction of poxviruses as cloning vectors: insertion of the thymidine kinase gene from herpes simplex virus into the DNA of infectious vaccinia virus. 1982. Biotechnology 24:503-7.

502. Panus, J. F., C. A. Smith, C. A. Ray, T. D. Smith, D. D. Patel, and D. J. Pickup. 2002. Cowpox virus encodes a fifth member of the tumor necrosis factor receptor family: a soluble, secreted CD30 homologue. Proc Natl Acad Sci U S A 99:8348-53.

503. Panzer, U., R. R. Reinking, O. M. Steinmetz, G. Zahner, U. Sudbeck, S. Fehr, B. Pfalzer, A. Schneider, F. Thaiss, M. Mack, S. Conrad, H. Huland, U. Helmchen, and R. A. Stahl. 2004. CXCR3 and CCR5 positive T-cell recruitment in acute human renal allograft rejection. Transplantation 78:1341-50.

504. Parish, C. R. 2005. Heparan sulfate and inflammation. Nat Immunol 6:861-2.

505. Parkinson, J. E., and G. L. Smith. 1994. Vaccinia virus gene A36R encodes a M(r) 43-50 K protein on the surface of extracellular enveloped virus. Virology 204:376-90.

506. Parrish, S., and B. Moss. 2006. Characterization of a vaccinia virus mutant with a deletion of the D10R gene encoding a putative negative regulator of gene expression. J Virol 80:553-61.

507. Patel, D. D., W. Koopmann, T. Imai, L. P. Whichard, O. Yoshie, and M. S. Krangel. 2001. Chemokines have diverse abilities to form solid phase gradients. Clin Immunol 99:43-52.

508. Patel, D. D., J. P. Zachariah, and L. P. Whichard. 2001. CXCR3 and CCR5 ligands in rheumatoid arthritis synovium. Clin Immunol 98:39-45.

509. Paxton, W. A., S. R. Martin, D. Tse, T. R. O'Brien, J. Skurnick, N. L. VanDevanter, N. Padian, J. F. Braun, D. P. Kotler, S. M. Wolinsky, and R. A. Koup. 1996. Relative resistance to HIV-1 infection of CD4 lymphocytes from persons who remain uninfected despite multiple high-risk sexual exposure. Nat Med 2:412-7.

510. Payne, L. G. 1979. Identification of the vaccinia hemagglutinin polypeptide from a cell system yielding large amounts of extracellular enveloped virus. J Virol 31:147-55.

511. Payne, L. G. 1980. Significance of extracellular enveloped virus in the in vitro and in vivo dissemination of vaccinia. J Gen Virol 50:89-100.

512. Payne, L. G., and K. Kristenson. 1979. Mechanism of vaccinia virus release and its specific inhibition by N1-isonicotinoyl-N2-3-methyl-4chlorobenzoylhydrazine. J Virol 32:614-22.

513. Payne, L. G., and K. Kristensson. 1982. The effect of cytochalasin D and monensin on enveloped vaccinia virus release. Arch Virol 74:11-20.

514. Payne, L. G., and E. Norrby. 1978. Adsorption and penetration of enveloped and naked vaccinia virus particles. J Virol 27:19-27.

515. Payne, L. G., and E. Norrby. 1976. Presence of haemagglutinin in the envelope of extracellular vaccinia virus particles. J Gen Virol 32:63-72. 
516. Pelkmans, L., and A. Helenius. 2002. Endocytosis via caveolae. Traffic 3:311-20.

517. Pello, O. M., L. Martinez-Munoz, V. Parrillas, A. Serrano, J. M. RodriguezFrade, M. J. Toro, P. Lucas, M. Monterrubio, A. C. Martinez, and M. Mellado. 2008. Ligand stabilization of CXCR4/delta-opioid receptor heterodimers reveals a mechanism for immune response regulation. Eur J Immunol 38:537-49.

518. Pierce, K. L., and R. J. Lefkowitz. 2001. Classical and new roles of betaarrestins in the regulation of G-protein-coupled receptors. Nat Rev Neurosci 2:727-33.

519. Piontek, G. E., R. Weltzin, and W. A. Tompkins. 1980. Enhanced cytotoxicity of mouse natural killer cells for vaccinia and herpes virusinfected targets. J Reticuloendothel Soc 27:175-88.

520. Plater-Zyberk, C., A. J. Hoogewerf, A. E. Proudfoot, C. A. Power, and T. N. Wells. 1997. Effect of a CC chemokine receptor antagonist on collagen induced arthritis in DBA/1 mice. Immunol Lett 57:117-20.

521. Pogo, B. G., S. M. Melana, and J. Blaho. 2004. Poxvirus infection and apoptosis. Int Rev Immunol 23:61-74.

522. Ponting, C. P., and I. D. Kerr. 1996. A novel family of phospholipase D homologues that includes phospholipid synthases and putative endonucleases: identification of duplicated repeats and potential active site residues. Protein Sci 5:914-22.

523. Potter, P., S. Tourdot, T. Blanchard, G. L. Smith, and K. G. Gould. 2001. Differential processing and presentation of the H-2D(b)-restricted epitope from two different strains of influenza virus nucleoprotein. J Gen Virol 82:1069-74.

524. Power, C. A. 2003. Knock out models to dissect chemokine receptor function in vivo. J Immunol Methods 273:73-82.

525. Prado, G. N., K. Suetomi, D. Shumate, C. Maxwell, A. Ravindran, K. Rajarathnam, and J. Navarro. 2007. Chemokine signaling specificity: essential role for the $\mathrm{N}$-terminal domain of chemokine receptors. Biochemistry 46:8961-8.

526. Proudfoot, A. E. 2002. Chemokine receptors: multifaceted therapeutic targets. Nat Rev Immunol 2:106-15.

527. Proudfoot, A. E. 2006. The biological relevance of chemokine-proteoglycan interactions. Biochem Soc Trans 34:422-6.

528. Proudfoot, A. E., S. Fritchley, F. Borlat, J. P. Shaw, F. Vilbois, C. Zwahlen, A. Trkola, D. Marchant, P. R. Clapham, and T. N. Wells. 2001. The BBXB motif of RANTES is the principal site for heparin binding and controls receptor selectivity. J Biol Chem 276:10620-6.

529. Proudfoot, A. E., T. M. Handel, Z. Johnson, E. K. Lau, P. LiWang, I. ClarkLewis, F. Borlat, T. N. Wells, and M. H. Kosco-Vilbois. 2003. Glycosaminoglycan binding and oligomerization are essential for the in vivo activity of certain chemokines. Proc Natl Acad Sci U S A 100:1885-90. 
530. Quan, L. T., A. Caputo, R. C. Bleackley, D. J. Pickup, and G. S. Salvesen. 1995. Granzyme B is inhibited by the cowpox virus serpin cytokine response modifier A. J Biol Chem 270:10377-9.

531. Racaniello, V. R. 1991. Virus-receptor interaction in poliovirus entry and pathogenesis. Harvey Lect 87:1-16.

532. Racaniello, V. R., and R. Ren. 1996. Poliovirus biology and pathogenesis. Curr Top Microbiol Immunol 206:305-25.

533. Racaniello, V. R., and R. Ren. 1994. Transgenic mice and the pathogenesis of poliomyelitis. Arch Virol Suppl 9:79-86.

534. Radin, J. N., C. J. Orihuela, G. Murti, C. Guglielmo, P. J. Murray, and E. I. Tuomanen. 2005. beta-Arrestin 1 participates in platelet-activating factor receptor-mediated endocytosis of Streptococcus pneumoniae. Infect Immun 73:7827-35.

535. Rahbar, R., T. T. Murooka, and E. N. Fish. 2008. A role for CCR5 in dissemination of vaccinia virus in vivo. J Virol.

536. Rahbar, R., T. T. Murooka, A. A. Hinek, C. L. Galligan, A. Sassano, C. Yu, K. Srivastava, L. C. Platanias, and E. N. Fish. 2006. Vaccinia virus activation of CCR5 invokes tyrosine phosphorylation signaling events that support virus replication. J Virol 80:7245-59.

537. Ramdin, L., B. Perks, N. Sheron, and J. K. Shute. 1998. Regulation of interleukin-8 binding and function by heparin and alpha2-macroglobulin. Clin Exp Allergy 28:616-24.

538. Ramesh, N., I. M. Anton, J. H. Hartwig, and R. S. Geha. 1997. WIP, a protein associated with wiskott-aldrich syndrome protein, induces actin polymerization and redistribution in lymphoid cells. Proc Natl Acad Sci U S A 94:14671-6.

539. Rao, A. R., M. Savithri Sukumar, S. Kamalakshi, T. V. Paramasivam, and S. Ramakrishnan. 1972. Further studies with precipitation in gel test in diagnosis of smallpox. I. Studies on detection of antibodies in sera by pig test. Indian J Med Res 60:1254-60.

540. Rao, A. R., M. S. Sukumar, T. V. Paramasivam, S. Kamalakshi, A. R. Parasuraman, and M. S. Ramakrishnan. 1971. Studies on viability of Variola virus. I. Viability of Variola virus in smallpox material on glass slides, in capillary tubes on filter paper and cotton threads. Indian J Med Res 59:699-704.

541. Raport, C. J., J. Gosling, V. L. Schweickart, P. W. Gray, and I. F. Charo. 1996. Molecular cloning and functional characterization of a novel human CC chemokine receptor (CCR5) for RANTES, MIP-1beta, and MIP-1alpha. J Biol Chem 271:17161-6.

542. Ray, C. A., R. A. Black, S. R. Kronheim, T. A. Greenstreet, P. R. Sleath, G. S. Salvesen, and D. J. Pickup. 1992. Viral inhibition of inflammation: cowpox virus encodes an inhibitor of the interleukin-1 beta converting enzyme. Cell 69:597-604. 
543. Reading, P. C., A. Khanna, and G. L. Smith. 2002. Vaccinia virus CrmE encodes a soluble and cell surface tumor necrosis factor receptor that contributes to virus virulence. Virology 292:285-98.

544. Reading, P. C., and G. L. Smith. 2003. A kinetic analysis of immune mediators in the lungs of mice infected with vaccinia virus and comparison with intradermal infection. J Gen Virol 84:1973-83.

545. Reading, P. C., J. A. Symons, and G. L. Smith. 2003. A soluble chemokinebinding protein from vaccinia virus reduces virus virulence and the inflammatory response to infection. J Immunol 170:1435-42.

546. Redfield, R. R., D. C. Wright, W. D. James, T. S. Jones, C. Brown, and D. S. Burke. 1987. Disseminated vaccinia in a military recruit with human immunodeficiency virus (HIV) disease. N Engl J Med 316:673-6.

547. Reece, J. C., A. J. Handley, E. J. Anstee, W. A. Morrison, S. M. Crowe, and P. U. Cameron. 1998. HIV-1 selection by epidermal dendritic cells during transmission across human skin. J Exp Med 187:1623-31.

548. Reeves, P. M., B. Bommarius, S. Lebeis, S. McNulty, J. Christensen, A. Swimm, A. Chahroudi, R. Chavan, M. B. Feinberg, D. Veach, W. Bornmann, M. Sherman, and D. Kalman. 2005. Disabling poxvirus pathogenesis by inhibition of Abl-family tyrosine kinases. Nat Med 11:731-9.

549. Ridley, A. J., and A. Hall. 1992. The small GTP-binding protein rho regulates the assembly of focal adhesions and actin stress fibers in response to growth factors. Cell 70:389-99.

550. Ridley, A. J., H. F. Paterson, C. L. Johnston, D. Diekmann, and A. Hall. 1992. The small GTP-binding protein rac regulates growth factor-induced membrane ruffling. Cell 70:401-10.

551. Rietdorf, J., A. Ploubidou, I. Reckmann, A. Holmstrom, F. Frischknecht, M. Zettl, T. Zimmermann, and M. Way. 2001. Kinesin-dependent movement on microtubules precedes actin-based motility of vaccinia virus. Nat Cell Biol 3:992-1000.

552. Rivero-Lezcano, O. M., A. Marcilla, J. H. Sameshima, and K. C. Robbins. 1995. Wiskott-Aldrich syndrome protein physically associates with Nck through Src homology 3 domains. Mol Cell Biol 15:5725-31.

553. Roberts, K. L., and G. L. Smith. 2008. Vaccinia virus morphogenesis and dissemination. Trends Microbiol 16:472-9.

554. Robertson, M. J. 2002. Role of chemokines in the biology of natural killer cells. J Leukoc Biol 71:173-83.

555. Robinson, E., E. C. Keystone, T. J. Schall, N. Gillett, and E. N. Fish. 1995. Chemokine expression in rheumatoid arthritis (RA): evidence of RANTES and macrophage inflammatory protein (MIP)-1 beta production by synovial $\mathrm{T}$ cells. Clin Exp Immunol 101:398-407.

556. Robinson, L. A., C. Nataraj, D. W. Thomas, D. N. Howell, R. Griffiths, V. Bautch, D. D. Patel, L. Feng, and T. M. Coffman. 2000. A role for fractalkine and its receptor (CX3CR1) in cardiac allograft rejection. J Immunol 165:6067-72. 
557. Rocheville, M., D. C. Lange, U. Kumar, S. C. Patel, R. C. Patel, and Y. C. Patel. 2000. Receptors for dopamine and somatostatin: formation of heterooligomers with enhanced functional activity. Science 288:154-7.

558. Rodger, G., and G. L. Smith. 2002. Replacing the SCR domains of vaccinia virus protein B5R with EGFP causes a reduction in plaque size and actin tail formation but enveloped virions are still transported to the cell surface. J Gen Virol 83:323-32.

559. Rodriguez, J. F., and G. L. Smith. 1990. IPTG-dependent vaccinia virus: identification of a virus protein enabling virion envelopment by Golgi membrane and egress. Nucleic Acids Res 18:5347-51.

560. Rodriguez-Frade, J. M., M. Mellado, and A. C. Martinez. 2001. Chemokine receptor dimerization: two are better than one. Trends Immunol 22:612-7.

561. Rodriguez-Frade, J. M., A. J. Vila-Coro, A. M. de Ana, J. P. Albar, A. C. Martinez, and M. Mellado. 1999. The chemokine monocyte chemoattractant protein-1 induces functional responses through dimerization of its receptor CCR2. Proc Natl Acad Sci U S A 96:3628-33.

562. Rodriguez-Frade, J. M., A. J. Vila-Coro, A. Martin, M. Nieto, F. SanchezMadrid, A. E. Proudfoot, T. N. Wells, A. C. Martinez, and M. Mellado. 1999. Similarities and differences in RANTES- and (AOP)-RANTES-triggered signals: implications for chemotaxis. J Cell Biol 144:755-65.

563. Rohatgi, R., P. Nollau, H. Y. Ho, M. W. Kirschner, and B. J. Mayer. 2001. Nck and phosphatidylinositol 4,5-bisphosphate synergistically activate actin polymerization through the N-WASP-Arp2/3 pathway. J Biol Chem 276:26448-52.

564. Rollins, B. J. 1997. Chemokines. Blood 90:909-28.

565. Roos, M. T., J. M. Lange, R. E. de Goede, R. A. Coutinho, P. T. Schellekens, F. Miedema, and M. Tersmette. 1992. Viral phenotype and immune response in primary human immunodeficiency virus type 1 infection. J Infect Dis 165:427-32.

566. Roper, R. L., L. G. Payne, and B. Moss. 1996. Extracellular vaccinia virus envelope glycoprotein encoded by the A33R gene. J Virol 70:3753-62.

567. Roper, R. L., E. J. Wolffe, A. Weisberg, and B. Moss. 1998. The envelope protein encoded by the A33R gene is required for formation of actincontaining microvilli and efficient cell-to-cell spread of vaccinia virus. J Virol 72:4192-204.

568. Rosales, R., N. Harris, B. Y. Ahn, and B. Moss. 1994. Purification and identification of a vaccinia virus-encoded intermediate stage promoterspecific transcription factor that has homology to eukaryotic transcription factor SII (TFIIS) and an additional role as a viral RNA polymerase subunit. J Biol Chem 269:14260-7.

569. Rosales, R., G. Sutter, and B. Moss. 1994. A cellular factor is required for transcription of vaccinia viral intermediate-stage genes. Proc Natl Acad Sci U S A 91:3794-8.

570. Rosemond, H., and B. Moss. 1973. Phosphoprotein component of vaccinia virions. J Virol 11:961-70. 
571. Rosen, O., J. Chill, M. Sharon, N. Kessler, B. Mester, S. Zolla-Pazner, and J. Anglister. 2005. Induced fit in HIV-neutralizing antibody complexes: evidence for alternative conformations of the gp120 V3 loop and the molecular basis for broad neutralization. Biochemistry 44:7250-8.

572. Rosengard, A. M., L. C. Alonso, L. C. Korb, W. M. Baldwin, 3rd, F. Sanfilippo, L. A. Turka, and J. M. Ahearn. 1999. Functional characterization of soluble and membrane-bound forms of vaccinia virus complement control protein (VCP). Mol Immunol 36:685-97.

573. Rosenkilde, M. M., T. N. Kledal, H. Brauner-Osborne, and T. W. Schwartz. 1999. Agonists and inverse agonists for the herpesvirus 8-encoded constitutively active seven-transmembrane oncogene product, ORF-74. J Biol Chem 274:956-61.

574. Rosenquist, G. L., and H. B. Nicholas, Jr. 1993. Analysis of sequence requirements for protein tyrosine sulfation. Protein Sci 2:215-22.

575. Roses, D. F. 1992. From Hunter and the Great Pox to Jenner and smallpox. Surg Gynecol Obstet 175:365-72.

576. Rossi, D., and A. Zlotnik. 2000. The biology of chemokines and their receptors. Annu Rev Immunol 18:217-42.

577. Rot, A. 1992. Endothelial cell binding of NAP-1/IL-8: role in neutrophil emigration. Immunol Today 13:291-4.

578. Rot, A. 1993. Neutrophil attractant/activation protein-1 (interleukin-8) induces in vitro neutrophil migration by haptotactic mechanism. Eur J Immunol 23:303-6.

579. Rottger, S., F. Frischknecht, I. Reckmann, G. L. Smith, and M. Way. 1999. Interactions between vaccinia virus IEV membrane proteins and their roles in IEV assembly and actin tail formation. J Virol 73:2863-75.

580. Roulston, A., R. C. Marcellus, and P. E. Branton. 1999. Viruses and apoptosis. Annu Rev Microbiol 53:577-628.

581. Roy, M., and R. M. Sapolsky. 2003. The neuroprotective effects of virallyderived caspase inhibitors p35 and crmA following a necrotic insult. Neurobiol Dis 14:1-9.

582. Ruffing, N., N. Sullivan, L. Sharmeen, J. Sodroski, and L. Wu. 1998. CCR5 has an expanded ligand-binding repertoire and is the primary receptor used by MCP-2 on activated T cells. Cell Immunol 189:160-8.

583. Ryan, C. A., H. R. Stennicke, V. E. Nava, J. B. Burch, J. M. Hardwick, and G. S. Salvesen. 2002. Inhibitor specificity of recombinant and endogenous caspase-9. Biochem J 366:595-601.

584. Sahu, A., S. N. Isaacs, A. M. Soulika, and J. D. Lambris. 1998. Interaction of vaccinia virus complement control protein with human complement proteins: factor I-mediated degradation of $\mathrm{C} 3 \mathrm{~b}$ to $\mathrm{iC} 3 \mathrm{~b} 1$ inactivates the alternative complement pathway. J Immunol 160:5596-604.

585. Salahpour, A., S. Angers, and M. Bouvier. 2000. Functional significance of oligomerization of G-protein-coupled receptors. Trends Endocrinol Metab 11:163-8. 
586. Salcedo, R., J. H. Resau, D. Halverson, E. A. Hudson, M. Dambach, D. Powell, K. Wasserman, and J. J. Oppenheim. 2000. Differential expression and responsiveness of chemokine receptors (CXCR1-3) by human microvascular endothelial cells and umbilical vein endothelial cells. FASEB J 14:2055-64.

587. Salmons, T., A. Kuhn, F. Wylie, S. Schleich, J. R. Rodriguez, D. Rodriguez, M. Esteban, G. Griffiths, and J. K. Locker. 1997. Vaccinia virus membrane proteins $\mathrm{p} 8$ and $\mathrm{p} 16$ are cotranslationally inserted into the rough endoplasmic reticulum and retained in the intermediate compartment. J Virol 71:7404-20.

588. Samson, M., O. Labbe, C. Mollereau, G. Vassart, and M. Parmentier. 1996. Molecular cloning and functional expression of a new human CC-chemokine receptor gene. Biochemistry 35:3362-7.

589. Samson, M., G. LaRosa, F. Libert, P. Paindavoine, M. Detheux, G. Vassart, and M. Parmentier. 1997. The second extracellular loop of CCR5 is the major determinant of ligand specificity. J Biol Chem 272:24934-41.

590. Samuel, C. E. 2001. Antiviral actions of interferons. Clin Microbiol Rev 14:778-809, table of contents.

591. Sanchez-Puig, J. M., L. Sanchez, G. Roy, and R. Blasco. 2004. Susceptibility of different leukocyte cell types to Vaccinia virus infection. Virol J 1:10.

592. Sanderson, C. M., F. Frischknecht, M. Way, M. Hollinshead, and G. L. Smith. 1998. Roles of vaccinia virus EEV-specific proteins in intracellular actin tail formation and low pH-induced cell-cell fusion. J Gen Virol 79 ( Pt 6):1415-25.

593. Sanderson, C. M., M. Way, and G. L. Smith. 1998. Virus-induced cell motility. J Virol 72:1235-43.

594. Sanz, P., and B. Moss. 1998. A new vaccinia virus intermediate transcription factor. J Virol 72:6880-3.

595. Saraiva, M., and A. Alcami. 2001. CrmE, a novel soluble tumor necrosis factor receptor encoded by poxviruses. J Virol 75:226-33.

596. Saraiva, M., P. Smith, P. G. Fallon, and A. Alcami. 2002. Inhibition of type 1 cytokine-mediated inflammation by a soluble CD30 homologue encoded by ectromelia (mousepox) virus. J Exp Med 196:829-39.

597. Scaplehorn, N., A. Holmstrom, V. Moreau, F. Frischknecht, I. Reckmann, and M. Way. 2002. Grb2 and Nck act cooperatively to promote actin-based motility of vaccinia virus. Curr Biol 12:740-5.

598. Schall, T. J. 1991. Biology of the RANTES/SIS cytokine family. Cytokine 3:165-83.

599. Schlecht, H. P., S. Schellhorn, B. J. Dezube, and J. M. Jacobson. 2008. New approaches in the treatment of HIV/AIDS - focus on maraviroc and other CCR5 antagonists. Ther Clin Risk Manag 4:473-85.

600. Schmelz, M., B. Sodeik, M. Ericsson, E. J. Wolffe, H. Shida, G. Hiller, and G. Griffiths. 1994. Assembly of vaccinia virus: the second wrapping cisterna is derived from the trans Golgi network. J Virol 68:130-47. 
601. Schneider-Schaulies, J. 2000. Cellular receptors for viruses: links to tropism and pathogenesis. J Gen Virol 81:1413-29.

602. Schreiber, M., L. Sedger, and G. McFadden. 1997. Distinct domains of M-T2, the myxoma virus tumor necrosis factor (TNF) receptor homolog, mediate extracellular TNF binding and intracellular apoptosis inhibition. J Virol 71:2171-81.

603. Schuh, J. M., K. Blease, and C. M. Hogaboam. 2002. The role of CC chemokine receptor 5 (CCR5) and RANTES/CCL5 during chronic fungal asthma in mice. FASEB J 16:228-30.

604. Schuitemaker, H., M. Koot, N. A. Kootstra, M. W. Dercksen, R. E. de Goede, R. P. van Steenwijk, J. M. Lange, J. K. Schattenkerk, F. Miedema, and M. Tersmette. 1992. Biological phenotype of human immunodeficiency virus type 1 clones at different stages of infection: progression of disease is associated with a shift from monocytotropic to T-cell-tropic virus population. J Virol 66:1354-60.

605. Schupp, P., M. Pfeffer, H. Meyer, G. Burck, K. Kolmel, and C. Neumann. 2001. Cowpox virus in a 12-year-old boy: rapid identification by an orthopoxvirus-specific polymerase chain reaction. Br J Dermatol 145:146-50.

606. Schwarz, D. A., C. D. Katayama, and S. M. Hedrick. 1998. Schlafen, a new family of growth regulatory genes that affect thymocyte development. Immunity 9:657-68.

607. Schweickart, V. L., A. Epp, C. J. Raport, and P. W. Gray. 2001. CCR11 is a functional receptor for the monocyte chemoaattractant protein family of chemokines. J Biol Chem 276:856.

608. Schweickart, V. L., A. Epp, C. J. Raport, and P. W. Gray. 2000. CCR11 is a functional receptor for the monocyte chemoattractant protein family of chemokines. J Biol Chem 275:9550-6.

609. Sedger, L., and G. McFadden. 1996. M-T2: a poxvirus TNF receptor homologue with dual activities. Immunol Cell Biol 74:538-45.

610. Seet, B. T., J. Barrett, J. Robichaud, B. Shilton, R. Singh, and G. McFadden. 2001. Glycosaminoglycan binding properties of the myxoma virus CCchemokine inhibitor, M-T1. J Biol Chem 276:30504-13.

611. Seet, B. T., J. B. Johnston, C. R. Brunetti, J. W. Barrett, H. Everett, C. Cameron, J. Sypula, S. H. Nazarian, A. Lucas, and G. McFadden. 2003. Poxviruses and immune evasion. Annu Rev Immunol 21:377-423.

612. Seet, B. T., and G. McFadden. 2002. Viral chemokine-binding proteins. J Leukoc Biol 72:24-34.

613. Sejvar, J. J., R. J. Labutta, L. E. Chapman, J. D. Grabenstein, J. Iskander, and J. M. Lane. 2005. Neurologic adverse events associated with smallpox vaccination in the United States, 2002-2004. JAMA 294:2744-50.

614. Sellebjerg, F., H. O. Madsen, C. V. Jensen, J. Jensen, and P. Garred. 2000. CCR5 delta32, matrix metalloproteinase- 9 and disease activity in multiple sclerosis. J Neuroimmunol 102:98-106.

615. Sen, G. C. 2001. Viruses and interferons. Annu Rev Microbiol 55:255-81. 
616. Senkevich, T. G., J. J. Bugert, J. R. Sisler, E. V. Koonin, G. Darai, and B. Moss. 1996. Genome sequence of a human tumorigenic poxvirus: prediction of specific host response-evasion genes. Science 273:813-6.

617. Senkevich, T. G., and B. Moss. 2005. Vaccinia virus H2 protein is an essential component of a complex involved in virus entry and cell-cell fusion. J Virol 79:4744-54.

618. Senkevich, T. G., S. Ojeda, A. Townsley, G. E. Nelson, and B. Moss. 2005. Poxvirus multiprotein entry-fusion complex. Proc Natl Acad Sci U S A 102:18572-7.

619. Senkevich, T. G., B. M. Ward, and B. Moss. 2004. Vaccinia virus A28L gene encodes an essential protein component of the virion membrane with intramolecular disulfide bonds formed by the viral cytoplasmic redox pathway. J Virol 78:2348-56.

620. Senkevich, T. G., B. M. Ward, and B. Moss. 2004. Vaccinia virus entry into cells is dependent on a virion surface protein encoded by the A28L gene. J Virol 78:2357-66.

621. Sharp, T. V., F. Moonan, A. Romashko, B. Joshi, G. N. Barber, and R. Jagus. 1998. The vaccinia virus E3L gene product interacts with both the regulatory and the substrate binding regions of PKR: implications for PKR autoregulation. Virology 250:302-15.

622. Shchelkunov, S. N., R. F. Massung, and J. J. Esposito. 1995. Comparison of the genome DNA sequences of Bangladesh-1975 and India-1967 variola viruses. Virus Res 36:107-18.

623. Shchelkunov, S. N., A. V. Totmenin, I. V. Babkin, P. F. Safronov, O. I. Ryazankina, N. A. Petrov, V. V. Gutorov, E. A. Uvarova, M. V. Mikheev, J. R. Sisler, J. J. Esposito, P. B. Jahrling, B. Moss, and L. S. Sandakhchiev. 2001. Human monkeypox and smallpox viruses: genomic comparison. FEBS Lett 509:66-70.

624. Shchelkunov, S. N., A. V. Totmenin, V. N. Loparev, P. F. Safronov, V. V. Gutorov, V. E. Chizhikov, J. C. Knight, J. M. Parsons, R. F. Massung, and J. J. Esposito. 2000. Alastrim smallpox variola minor virus genome DNA sequences. Virology 266:361-86.

625. Shida, H. 1986. Nucleotide sequence of the vaccinia virus hemagglutinin gene. Virology 150:451-62.

626. Shisler, J. L., and B. Moss. 2001. Immunology 102 at poxvirus U: avoiding apoptosis. Semin Immunol 13:67-72.

627. Shisler, J. L., and B. Moss. 2001. Molluscum contagiosum virus inhibitors of apoptosis: The MC159 v-FLIP protein blocks Fas-induced activation of procaspases and degradation of the related MC160 protein. Virology 282:1425.

628. Signoret, N., L. Hewlett, S. Wavre, A. Pelchen-Matthews, M. Oppermann, and M. Marsh. 2005. Agonist-induced endocytosis of CC chemokine receptor 5 is clathrin dependent. Mol Biol Cell 16:902-17. 
629. Signoret, N., A. Pelchen-Matthews, M. Mack, A. E. Proudfoot, and M. Marsh. 2000. Endocytosis and recycling of the HIV coreceptor CCR5. J Cell Biol 151:1281-94.

630. Simmons, G., J. D. Reeves, S. Hibbitts, J. T. Stine, P. W. Gray, A. E. Proudfoot, and P. R. Clapham. 2000. Co-receptor use by HIV and inhibition of HIV infection by chemokine receptor ligands. Immunol Rev 177:112-26.

631. Sirois, S., T. Sing, and K. C. Chou. 2005. HIV-1 gp120 V3 loop for structurebased drug design. Curr Protein Pept Sci 6:413-22.

632. Smith, C. A., T. Davis, J. M. Wignall, W. S. Din, T. Farrah, C. Upton, G. McFadden, and R. G. Goodwin. 1991. T2 open reading frame from the Shope fibroma virus encodes a soluble form of the TNF receptor. Biochem Biophys Res Commun 176:335-42.

633. Smith, C. A., T. D. Smith, P. J. Smolak, D. Friend, H. Hagen, M. Gerhart, L. Park, D. J. Pickup, D. Torrance, K. Mohler, K. Schooley, and R. G. Goodwin. 1997. Poxvirus genomes encode a secreted, soluble protein that preferentially inhibits beta chemokine activity yet lacks sequence homology to known chemokine receptors. Virology 236:316-27.

634. Smith, E. J., I. Marie, A. Prakash, A. Garcia-Sastre, and D. E. Levy. 2001. IRF3 and IRF7 phosphorylation in virus-infected cells does not require double-stranded RNA-dependent protein kinase R or Ikappa B kinase but is blocked by Vaccinia virus E3L protein. J Biol Chem 276:8951-7.

635. Smith, G. L., and Y. S. Chan. 1991. Two vaccinia virus proteins structurally related to the interleukin-1 receptor and the immunoglobulin superfamily. J Gen Virol 72 ( Pt 3):511-8.

636. Smith, G. L., J. A. Symons, A. Khanna, A. Vanderplasschen, and A. Alcami. 1997. Vaccinia virus immune evasion. Immunol Rev 159:137-54.

637. Smith, G. L., and A. Vanderplasschen. 1998. Extracellular enveloped vaccinia virus. Entry, egress, and evasion. Adv Exp Med Biol 440:395-414.

638. Smith, G. L., A. Vanderplasschen, and M. Law. 2002. The formation and function of extracellular enveloped vaccinia virus. J Gen Virol 83:2915-31.

639. Smith, S. A., and G. J. Kotwal. 2002. Immune response to poxvirus infections in various animals. Crit Rev Microbiol 28:149-85.

640. Smith, S. A., N. P. Mullin, J. Parkinson, S. N. Shchelkunov, A. V. Totmenin, V. N. Loparev, R. Srisatjaluk, D. N. Reynolds, K. L. Keeling, D. E. Justus, P. N. Barlow, and G. J. Kotwal. 2000. Conserved surface-exposed K/R-X-K/R motifs and net positive charge on poxvirus complement control proteins serve as putative heparin binding sites and contribute to inhibition of molecular interactions with human endothelial cells: a novel mechanism for evasion of host defense. J Virol 74:5659-66.

641. Smith, V. P., and A. Alcami. 2000. Expression of secreted cytokine and chemokine inhibitors by ectromelia virus. J Virol 74:8460-71.

642. Smith, V. P., N. A. Bryant, and A. Alcami. 2000. Ectromelia, vaccinia and cowpox viruses encode secreted interleukin-18-binding proteins. J Gen Virol 81:1223-30. 
643. Sodeik, B., R. W. Doms, M. Ericsson, G. Hiller, C. E. Machamer, W. van 't Hof, G. van Meer, B. Moss, and G. Griffiths. 1993. Assembly of vaccinia virus: role of the intermediate compartment between the endoplasmic reticulum and the Golgi stacks. J Cell Biol 121:521-41.

644. Sodeik, B., and J. Krijnse-Locker. 2002. Assembly of vaccinia virus revisited: de novo membrane synthesis or acquisition from the host? Trends Microbiol 10:15-24.

645. Sodek, K. L., A. I. Evangelou, A. Ignatchenko, M. Agochiya, T. J. Brown, M. J. Ringuette, I. Jurisica, and T. Kislinger. 2008. Identification of pathways associated with invasive behavior by ovarian cancer cells using multidimensional protein identification technology (MudPIT). Mol Biosyst 4:762-73.

646. Sohy, D., M. Parmentier, and J. Y. Springael. 2007. Allosteric transinhibition by specific antagonists in CCR2/CXCR4 heterodimers. J Biol Chem 282:30062-9.

647. Song, M., C. M. Breneman, and N. Sukumar. 2004. Three-dimensional quantitative structure-activity relationship analyses of piperidine-based CCR5 receptor antagonists. Bioorg Med Chem 12:489-99.

648. Spillmann, D., D. Witt, and U. Lindahl. 1998. Defining the interleukin-8binding domain of heparan sulfate. J Biol Chem 273:15487-93.

649. Spriggs, M. K. 1996. One step ahead of the game: viral immunomodulatory molecules. Annu Rev Immunol 14:101-30.

650. Spriggs, M. K., D. E. Hruby, C. R. Maliszewski, D. J. Pickup, J. E. Sims, R. M. Buller, and J. VanSlyke. 1992. Vaccinia and cowpox viruses encode a novel secreted interleukin-1-binding protein. Cell 71:145-52.

651. Springael, J. Y., P. N. Le Minh, E. Urizar, S. Costagliola, G. Vassart, and M. Parmentier. 2006. Allosteric modulation of binding properties between units of chemokine receptor homo- and hetero-oligomers. Mol Pharmacol 69:1652-61.

652. Sroller, V., V. Ludvikova, L. Maresova, P. Hainz, and S. Nemeckova. 2001. Effect of IFN-gamma receptor gene deletion on vaccinia virus virulence. Arch Virol 146:239-49.

653. Stein, B. S., S. D. Gowda, J. D. Lifson, R. C. Penhallow, K. G. Bensch, and E. G. Engleman. 1987. pH-independent HIV entry into CD4-positive T cells via virus envelope fusion to the plasma membrane. Cell 49:659-68.

654. Stephens, J. C., D. E. Reich, D. B. Goldstein, H. D. Shin, M. W. Smith, M. Carrington, C. Winkler, G. A. Huttley, R. Allikmets, L. Schriml, B. Gerrard, M. Malasky, M. D. Ramos, S. Morlot, M. Tzetis, C. Oddoux, F. S. di Giovine, G. Nasioulas, D. Chandler, M. Aseev, M. Hanson, L. Kalaydjieva, D. Glavac, P. Gasparini, E. Kanavakis, M. Claustres, M. Kambouris, H. Ostrer, G. Duff, V. Baranov, H. Sibul, A. Metspalu, D. Goldman, N. Martin, D. Duffy, J. Schmidtke, X. Estivill, S. J. O'Brien, and M. Dean. 1998. Dating the origin of the CCR5-Delta32 AIDS-resistance allele by the coalescence of haplotypes. Am J Hum Genet 62:1507-15. 
655. Stephens, L. R., A. Eguinoa, H. Erdjument-Bromage, M. Lui, F. Cooke, J. Coadwell, A. S. Smrcka, M. Thelen, K. Cadwallader, P. Tempst, and P. T. Hawkins. 1997. The G beta gamma sensitivity of a PI3K is dependent upon a tightly associated adaptor, p101. Cell 89:105-14.

656. Stern, W., and S. Dales. 1974. Biogenesis of vaccinia: concerning the origin of the envelope phospholipids. Virology 62:293-306.

657. Stern, W., and S. Dales. 1976. Biogenesis of vaccinia: relationship of the envelope to virus assembly. Virology 75:242-55.

658. Stewart, P. L., and G. R. Nemerow. 1997. Recent structural solutions for antibody neutralization of viruses. Trends Microbiol 5:229-33.

659. Stoyanov, B., S. Volinia, T. Hanck, I. Rubio, M. Loubtchenkov, D. Malek, S. Stoyanova, B. Vanhaesebroeck, R. Dhand, B. Nurnberg, and et al. 1995. Cloning and characterization of a $\mathrm{G}$ protein-activated human phosphoinositide-3 kinase. Science 269:690-3.

660. Superti, F., L. Seganti, F. M. Ruggeri, A. Tinari, G. Donelli, and N. Orsi. 1987. Entry pathway of vesicular stomatitis virus into different host cells. J Gen Virol 68 ( Pt 2):387-99.

661. Suzaki, Y., K. Hamada, T. Nomi, T. Ito, M. Sho, Y. Kai, Y. Nakajima, and H. Kimura. 2008. A small-molecule compound targeting CCR5 and CXCR3 prevents airway hyperresponsiveness and inflammation. Eur Respir J 31:783-9.

662. Suzuki, N., A. Nakajima, S. Yoshino, K. Matsushima, H. Yagita, and K. Okumura. 1999. Selective accumulation of CCR5+ T lymphocytes into inflamed joints of rheumatoid arthritis. Int Immunol 11:553-9.

663. Suzuki, S., L. F. Chuang, P. Yau, R. H. Doi, and R. Y. Chuang. 2002. Interactions of opioid and chemokine receptors: oligomerization of $\mathrm{mu}$, kappa, and delta with CCR5 on immune cells. Exp Cell Res 280:192-200.

664. Symons, J. A., E. Adams, D. C. Tscharke, P. C. Reading, H. Waldmann, and G. L. Smith. 2002. The vaccinia virus C12L protein inhibits mouse IL-18 and promotes virus virulence in the murine intranasal model. J Gen Virol 83:2833-44.

665. Symons, J. A., A. Alcami, and G. L. Smith. 1995. Vaccinia virus encodes a soluble type I interferon receptor of novel structure and broad species specificity. Cell 81:551-60.

666. Symons, J. A., D. C. Tscharke, N. Price, and G. L. Smith. 2002. A study of the vaccinia virus interferon-gamma receptor and its contribution to virus virulence. J Gen Virol 83:1953-64.

667. Symons, M., J. M. Derry, B. Karlak, S. Jiang, V. Lemahieu, F. McCormick, U. Francke, and A. Abo. 1996. Wiskott-Aldrich syndrome protein, a novel effector for the GTPase CDC42Hs, is implicated in actin polymerization. Cell 84:723-34.

668. Szabo, M. C., K. S. Soo, A. Zlotnik, and T. J. Schall. 1995. Chemokine class differences in binding to the Duffy antigen-erythrocyte chemokine receptor. J Biol Chem 270:25348-51. 
669. Szczucinski, A., and J. Losy. 2007. Chemokines and chemokine receptors in multiple sclerosis. Potential targets for new therapies. Acta Neurol Scand 115:137-46.

670. Tagaya, I., T. Kitamura, and Y. Sano. 1961. A new mutant of dermovaccinia virus. Nature 192:381-2.

671. Takahashi-Nishimaki, F., S. Funahashi, K. Miki, S. Hashizume, and M. Sugimoto. 1991. Regulation of plaque size and host range by a vaccinia virus gene related to complement system proteins. Virology 181:158-64.

672. Tanaka, Y., D. H. Adams, S. Hubscher, H. Hirano, U. Siebenlist, and S. Shaw. 1993. T-cell adhesion induced by proteoglycan-immobilized cytokine MIP-1 beta. Nature 361:79-82.

673. Tennyson, H. C., and E. A. Mair. 2004. Smallpox: what every otolaryngologist should know. Otolaryngol Head Neck Surg 130:323-33.

674. Terricabras, E., C. Benjamim, and N. Godessart. 2004. Drug discovery and chemokine receptor antagonists: eppur si muove! Autoimmun Rev 3:550-6.

675. Terrillon, S., T. Durroux, B. Mouillac, A. Breit, M. A. Ayoub, M. Taulan, R. Jockers, C. Barberis, and M. Bouvier. 2003. Oxytocin and vasopressin V1a and $\mathrm{V} 2$ receptors form constitutive homo- and heterodimers during biosynthesis. Mol Endocrinol 17:677-91.

676. Tewari, M., and V. M. Dixit. 1995. Fas- and tumor necrosis factor-induced apoptosis is inhibited by the poxvirus crmA gene product. J Biol Chem 270:3255-60.

677. Tewari, M., L. T. Quan, K. O'Rourke, S. Desnoyers, Z. Zeng, D. R. Beidler, G. G. Poirier, G. S. Salvesen, and V. M. Dixit. 1995. Yama/CPP32 beta, a mammalian homolog of CED-3, is a CrmA-inhibitable protease that cleaves the death substrate poly(ADP-ribose) polymerase. Cell 81:801-9.

678. Thome, M., P. Schneider, K. Hofmann, H. Fickenscher, E. Meinl, F. Neipel, C. Mattmann, K. Burns, J. L. Bodmer, M. Schroter, C. Scaffidi, P. H. Krammer, M. E. Peter, and J. Tschopp. 1997. Viral FLICE-inhibitory proteins (FLIPs) prevent apoptosis induced by death receptors. Nature 386:517-21.

679. Tooze, J., M. Hollinshead, B. Reis, K. Radsak, and H. Kern. 1993. Progeny vaccinia and human cytomegalovirus particles utilize early endosomal cisternae for their envelopes. Eur J Cell Biol 60:163-78.

680. Townsend, A., J. Bastin, K. Gould, G. Brownlee, M. Andrew, B. Coupar, D. Boyle, S. Chan, and G. Smith. 1988. Defective presentation to class Irestricted cytotoxic $\mathrm{T}$ lymphocytes in vaccinia-infected cells is overcome by enhanced degradation of antigen. J Exp Med 168:1211-24.

681. Townsley, A. C., T. G. Senkevich, and B. Moss. 2005. The product of the vaccinia virus L5R gene is a fourth membrane protein encoded by all poxviruses that is required for cell entry and cell-cell fusion. J Virol 79:10988-98.

682. Townsley, A. C., T. G. Senkevich, and B. Moss. 2005. Vaccinia virus A21 virion membrane protein is required for cell entry and fusion. J Virol 79:9458-69. 
683. Townson, J. R., and R. J. Nibbs. 2002. Characterization of mouse CCX-CKR, a receptor for the lymphocyte-attracting chemokines TECK/mCCL25, SLC/mCCL21 and MIP-3beta/mCCL19: comparison to human CCX-CKR. Eur J Immunol 32:1230-41.

684. Tripp, R. A., L. P. Jones, L. M. Haynes, H. Zheng, P. M. Murphy, and L. J. Anderson. 2001. CX3C chemokine mimicry by respiratory syncytial virus $G$ glycoprotein. Nat Immunol 2:732-8.

685. Trkola, A., T. J. Ketas, K. A. Nagashima, L. Zhao, T. Cilliers, L. Morris, J. P. Moore, P. J. Maddon, and W. C. Olson. 2001. Potent, broad-spectrum inhibition of human immunodeficiency virus type 1 by the CCR5 monoclonal antibody PRO 140. J Virol 75:579-88.

686. Tsuji, Y., Y. Shimada, T. Takeshita, N. Kajimura, S. Nomura, N. Sekiyama, J. Otomo, J. Usukura, S. Nakanishi, and H. Jingami. 2000. Cryptic dimer interface and domain organization of the extracellular region of metabotropic glutamate receptor subtype 1. J Biol Chem 275:28144-51.

687. Tsutsui, H., K. Nakanishi, K. Matsui, K. Higashino, H. Okamura, Y. Miyazawa, and K. Kaneda. 1996. IFN-gamma-inducing factor up-regulates Fas ligand-mediated cytotoxic activity of murine natural killer cell clones. J Immunol 157:3967-73.

688. Tsutsui, K. 1983. Release of vaccinia virus from FL cells infected with the IHD-W strain. J Electron Microsc (Tokyo) 32:125-40.

689. Tsutsui, K., F. Uno, K. Akatsuka, and S. Nii. 1983. Electron microscopic study on vaccinia virus release. Arch Virol 75:213-8.

690. Turner, G. S. 1967. Respiratory infection of mice with vaccinia virus. J Gen Virol 1:399-402.

691. Turner, P. C., and R. W. Moyer. 2002. Poxvirus immune modulators: functional insights from animal models. Virus Res 88:35-53.

692. Turner, P. C., M. C. Sancho, S. R. Thoennes, A. Caputo, R. C. Bleackley, and R. W. Moyer. 1999. Myxoma virus Serp2 is a weak inhibitor of granzyme B and interleukin-1beta-converting enzyme in vitro and unlike CrmA cannot block apoptosis in cowpox virus-infected cells. J Virol 73:6394-404.

693. Ubogu, E. E., M. B. Cossoy, and R. M. Ransohoff. 2006. The expression and function of chemokines involved in CNS inflammation. Trends Pharmacol Sci 27:48-55.

694. Ueda, Y., M. Ito, and I. Tagaya. 1969. A specific surface antigen induced by poxvirus. Virology 38:180-2.

695. Ueda, Y., S. Morikawa, and Y. Matsuura. 1990. Identification and nucleotide sequence of the gene encoding a surface antigen induced by vaccinia virus. Virology 177:588-94.

696. Ueda, Y., I. Tagaya, H. Amano, and M. Ito. 1972. Studies on the early antigens induced by vaccinia virus. Virology 49:794-800.

697. Uetz, P., L. Giot, G. Cagney, T. A. Mansfield, R. S. Judson, J. R. Knight, D. Lockshon, V. Narayan, M. Srinivasan, P. Pochart, A. Qureshi-Emili, Y. Li, B. Godwin, D. Conover, T. Kalbfleisch, G. Vijayadamodar, M. Yang, M. 
Johnston, S. Fields, and J. M. Rothberg. 2000. A comprehensive analysis of protein-protein interactions in Saccharomyces cerevisiae. Nature 403:623-7.

698. Upton, C., J. L. Macen, M. Schreiber, and G. McFadden. 1991. Myxoma virus expresses a secreted protein with homology to the tumor necrosis factor receptor gene family that contributes to viral virulence. Virology 184:370-82.

699. Upton, C., K. Mossman, and G. McFadden. 1992. Encoding of a homolog of the IFN-gamma receptor by myxoma virus. Science 258:1369-72.

700. Valderrama, F., J. V. Cordeiro, S. Schleich, F. Frischknecht, and M. Way. 2006. Vaccinia virus-induced cell motility requires F11L-mediated inhibition of RhoA signaling. Science 311:377-81.

701. van Eijl, H., M. Hollinshead, G. Rodger, W. H. Zhang, and G. L. Smith. 2002. The vaccinia virus F12L protein is associated with intracellular enveloped virus particles and is required for their egress to the cell surface. J Gen Virol 83:195-207.

702. van Eijl, H., M. Hollinshead, and G. L. Smith. 2000. The vaccinia virus A36R protein is a type Ib membrane protein present on intracellular but not extracellular enveloped virus particles. Virology 271:26-36.

703. Vancova, I., C. La Bonnardiere, and P. Kontsek. 1998. Vaccinia virus protein B18R inhibits the activity and cellular binding of the novel type interferon-delta. J Gen Virol 79 ( Pt 7):1647-9.

704. Vandekerckhove, L., C. Verhofstede, and D. Vogelaers. 2008. Maraviroc: integration of a new antiretroviral drug class into clinical practice. J Antimicrob Chemother 61:1187-90.

705. Vanderplasschen, A., M. Hollinshead, and G. L. Smith. 1998. Intracellular and extracellular vaccinia virions enter cells by different mechanisms. J Gen Virol 79 ( Pt 4):877-87.

706. Vanderplasschen, A., E. Mathew, M. Hollinshead, R. B. Sim, and G. L. Smith. 1998. Extracellular enveloped vaccinia virus is resistant to complement because of incorporation of host complement control proteins into its envelope. Proc Natl Acad Sci U S A 95:7544-9.

707. Vanderplasschen, A., and G. L. Smith. 1997. A novel virus binding assay using confocal microscopy: demonstration that the intracellular and extracellular vaccinia virions bind to different cellular receptors. J Virol 71:4032-41.

708. VanSlyke, J. K., and D. E. Hruby. 1990. Posttranslational modification of vaccinia virus proteins. Curr Top Microbiol Immunol 163:185-206.

709. Vazquez-Salat, N., N. Yuhki, T. Beck, S. J. O'Brien, and W. J. Murphy. 2007. Gene conversion between mammalian CCR2 and CCR5 chemokine receptor genes: a potential mechanism for receptor dimerization. Genomics 90:21324.

710. Venkatesan, S., A. Petrovic, M. Locati, Y. O. Kim, D. Weissman, and P. M. Murphy. 2001. A membrane-proximal basic domain and cysteine cluster in the C-terminal tail of CCR5 constitute a bipartite motif critical for cell surface expression. J Biol Chem 276:40133-45. 
711. Venkatesan, S., J. J. Rose, R. Lodge, P. M. Murphy, and J. F. Foley. 2003. Distinct mechanisms of agonist-induced endocytosis for human chemokine receptors CCR5 and CXCR4. Mol Biol Cell 14:3305-24.

712. Vicari, A. P., and C. Caux. 2002. Chemokines in cancer. Cytokine Growth Factor Rev 13:143-54.

713. Vidricaire, G., and M. J. Tremblay. 2005. Rab5 and Rab7, but not ARF6, govern the early events of HIV-1 infection in polarized human placental cells. J Immunol 175:6517-30.

714. Vila-Coro, A. J., M. Mellado, A. Martin de Ana, A. C. Martinez, and J. M. Rodriguez-Frade. 1999. Characterization of RANTES- and aminooxypentane-RANTES-triggered desensitization signals reveals differences in recruitment of the G protein-coupled receptor complex. J Immunol 163:3037-44.

715. Vila-Coro, A. J., J. M. Rodriguez-Frade, A. Martin De Ana, M. C. MorenoOrtiz, A. C. Martinez, and M. Mellado. 1999. The chemokine SDF-1alpha triggers CXCR4 receptor dimerization and activates the JAK/STAT pathway. FASEB J 13:1699-710.

716. Volin, M. V., M. R. Shah, M. Tokuhira, G. K. Haines, J. M. Woods, and A. E. Koch. 1998. RANTES expression and contribution to monocyte chemotaxis in arthritis. Clin Immunol Immunopathol 89:44-53.

717. Vonderheit, A., and A. Helenius. 2005. Rab7 associates with early endosomes to mediate sorting and transport of Semliki forest virus to late endosomes. PLoS Biol 3:e233.

718. Vos, J. C., M. Sasker, and H. G. Stunnenberg. 1991. Promoter melting by a stage-specific vaccinia virus transcription factor is independent of the presence of RNA polymerase. Cell 65:105-13.

719. Vos, J. C., M. Sasker, and H. G. Stunnenberg. 1991. Vaccinia virus capping enzyme is a transcription initiation factor. EMBO J 10:2553-8.

720. Wagenaar, T. R., and B. Moss. 2007. Association of vaccinia virus fusion regulatory proteins with the multicomponent entry/fusion complex. J Virol 81:6286-93.

721. Wagner, L., O. O. Yang, E. A. Garcia-Zepeda, Y. Ge, S. A. Kalams, B. D. Walker, M. S. Pasternack, and A. D. Luster. 1998. Beta-chemokines are released from HIV-1-specific cytolytic T-cell granules complexed to proteoglycans. Nature 391:908-11.

722. Wali, A., and D. S. Strayer. 1999. Comparative effects of virulent and avirulent poxviruses on cell cycle progression. Exp Mol Pathol 66:31-8.

723. Wali, A., and D. S. Strayer. 1999. Infection with vaccinia virus alters regulation of cell cycle progression. DNA Cell Biol 18:837-43.

724. Wali, A., and D. S. Strayer. 1996. Regulation of p53 gene expression by a poxviral transcription factor. Virology 224:63-72.

725. Wang, F., Y. Ma, J. W. Barrett, X. Gao, J. Loh, E. Barton, H. W. Virgin, and G. McFadden. 2004. Disruption of Erk-dependent type I interferon induction breaks the myxoma virus species barrier. Nat Immunol 5:1266-74. 
726. Wang, J., X. Chenivesse, B. Henglein, and C. Brechot. 1990. Hepatitis B virus integration in a cyclin A gene in a hepatocellular carcinoma. Nature 343:555-7.

727. Wang, J., F. Zindy, X. Chenivesse, E. Lamas, B. Henglein, and C. Brechot. 1992. Modification of cyclin A expression by hepatitis B virus DNA integration in a hepatocellular carcinoma. Oncogene 7:1653-6.

728. Wang, L. H., T. C. Sudhof, and R. G. Anderson. 1995. The appendage domain of alpha-adaptin is a high affinity binding site for dynamin. J Biol Chem 270:10079-83.

729. Ward, B. M. 2005. Visualization and characterization of the intracellular movement of vaccinia virus intracellular mature virions. J Virol 79:4755-63.

730. Ward, B. M., and B. Moss. 2000. Golgi network targeting and plasma membrane internalization signals in vaccinia virus B5R envelope protein. J Virol 74:3771-80.

731. Ward, B. M., and B. Moss. 2001. Vaccinia virus intracellular movement is associated with microtubules and independent of actin tails. J Virol 75:11651-63.

732. Ward, B. M., and B. Moss. 2001. Visualization of intracellular movement of vaccinia virus virions containing a green fluorescent protein-B5R membrane protein chimera. J Virol 75:4802-13.

733. Ward, S. V., and C. E. Samuel. 2003. The PKR kinase promoter binds both Sp1 and Sp3, but only Sp3 functions as part of the interferon-inducible complex with ISGF-3 proteins. Virology 313:553-66.

734. Wareing, M. D., A. B. Lyon, B. Lu, C. Gerard, and S. R. Sarawar. 2004. Chemokine expression during the development and resolution of a pulmonary leukocyte response to influenza A virus infection in mice. J Leukoc Biol 76:886-95.

735. Watson B, A. S. 1994. The G-Protein Linked Receptor Facts Book. Academic Press, London.

736. Watson, C., S. Jenkinson, W. Kazmierski, and T. Kenakin. 2005. The CCR5 receptor-based mechanism of action of 873140 , a potent allosteric noncompetitive HIV entry inhibitor. Mol Pharmacol 67:1268-82.

737. Webb, L. M., and A. Alcami. 2005. Virally encoded chemokine binding proteins. Mini Rev Med Chem 5:833-48.

738. Webb, L. M., M. U. Ehrengruber, I. Clark-Lewis, M. Baggiolini, and A. Rot. 1993. Binding to heparan sulfate or heparin enhances neutrophil responses to interleukin 8. Proc Natl Acad Sci U S A 90:7158-62.

739. Weisswange, I., T. P. Newsome, S. Schleich, and M. Way. 2009. The rate of N-WASP exchange limits the extent of ARP2/3-complex-dependent actinbased motility. Nature 458:87-91.

740. Welch, M. D., A. H. DePace, S. Verma, A. Iwamatsu, and T. J. Mitchison. 1997. The human Arp2/3 complex is composed of evolutionarily conserved subunits and is localized to cellular regions of dynamic actin filament assembly. J Cell Biol 138:375-84. 
741. Welch, P. J., and J. Y. Wang. 1992. Coordinated synthesis and degradation of cdc2 in the mammalian cell cycle. Proc Natl Acad Sci U S A 89:3093-7.

742. Werden, S. J., M. M. Rahman, and G. McFadden. 2008. Poxvirus host range genes. Adv Virus Res 71:135-71.

743. White, J. H., A. Wise, M. J. Main, A. Green, N. J. Fraser, G. H. Disney, A. A. Barnes, P. Emson, S. M. Foord, and F. H. Marshall. 1998.

Heterodimerization is required for the formation of a functional GABA(B) receptor. Nature 396:679-82.

744. White, M. F. 1998. The IRS-signaling system: a network of docking proteins that mediate insulin and cytokin action. Recent Prog Horm Res. 53:119-38.

745. Wiertz, E. J., T. R. Jones, L. Sun, M. Bogyo, H. J. Geuze, and H. L. Ploegh. 1996. The human cytomegalovirus US11 gene product dislocates MHC class I heavy chains from the endoplasmic reticulum to the cytosol. Cell 84:769-79.

746. Willer, D. O., G. McFadden, and D. H. Evans. 1999. The complete genome sequence of shope (rabbit) fibroma virus. Virology 264:319-43.

747. Williams, G. 1988. WHO--the days of the mass campaigns. World Health Forum 9:7-23.

748. Williamson, J. D., R. W. Reith, L. J. Jeffrey, J. R. Arrand, and M. Mackett. 1990. Biological characterization of recombinant vaccinia viruses in mice infected by the respiratory route. J Gen Virol 71 ( Pt 11):2761-7.

749. Wimmer, E. 1994. Cellular receptors for animal viruses. Cold Spring Harbor Laboratory Press, Cold Spring Harbor, N.Y.

750. Witt, D. P., and A. D. Lander. 1994. Differential binding of chemokines to glycosaminoglycan subpopulations. Curr Biol 4:394-400.

751. Wolffe, E. J., S. N. Isaacs, and B. Moss. 1993. Deletion of the vaccinia virus B5R gene encoding a 42-kilodalton membrane glycoprotein inhibits extracellular virus envelope formation and dissemination. J Virol 67:473241.

752. Wolffe, E. J., S. Vijaya, and B. Moss. 1995. A myristylated membrane protein encoded by the vaccinia virus L1R open reading frame is the target of potent neutralizing monoclonal antibodies. Virology 211:53-63.

753. Wolffe, E. J., A. S. Weisberg, and B. Moss. 1998. Role for the vaccinia virus A36R outer envelope protein in the formation of virus-tipped actincontaining microvilli and cell-to-cell virus spread. Virology 244:20-6.

754. Wolffe, E. J., A. S. Weisberg, and B. Moss. 2001. The vaccinia virus A33R protein provides a chaperone function for viral membrane localization and tyrosine phosphorylation of the A36R protein. J Virol 75:303-10.

755. Wolfs, T. F., J. A. Wagenaar, H. G. Niesters, and A. D. Osterhaus. 2002. Ratto-human transmission of Cowpox infection. Emerg Infect Dis 8:1495-6.

756. Wong, M., and E. N. Fish. 1998. RANTES and MIP-1alpha activate stats in T cells. J Biol Chem 273:309-14.

757. Wong, M., S. Uddin, B. Majchrzak, T. Huynh, A. E. Proudfoot, L. C. Platanias, and E. N. Fish. 2001. Rantes activates Jak2 and Jak3 to regulate engagement of multiple signaling pathways in T cells. J Biol Chem 276:11427-31. 
758. Wong, M. M., and E. N. Fish. 2003. Chemokines: attractive mediators of the immune response. Semin Immunol 15:5-14.

759. Wong, M. M., Fish, EN. 2003. Chemokines: attractive mediators of the immune response. Semin immunol. 15:5-14.

760. Wood, A., and D. Armour. 2005. The discovery of the CCR5 receptor antagonist, UK-427,857, a new agent for the treatment of HIV infection and AIDS. Prog Med Chem 43:239-71.

761. Wucherpfennig, K. W., and J. L. Strominger. 1995. Selective binding of self peptides to disease-associated major histocompatibility complex (MHC) molecules: a mechanism for MHC-linked susceptibility to human autoimmune diseases. J Exp Med 181:1597-601.

762. Wurch, T., A. Matsumoto, and P. J. Pauwels. 2001. Agonist-independent and -dependent oligomerization of dopamine $\mathrm{D}(2)$ receptors by fusion to fluorescent proteins. FEBS Lett 507:109-13.

763. Wyatt, L. S., M. W. Carroll, C. P. Czerny, M. Merchlinsky, J. R. Sisler, and B. Moss. 1998. Marker rescue of the host range restriction defects of modified vaccinia virus Ankara. Virology 251:334-42.

764. Wyatt, L. S., P. L. Earl, L. A. Eller, and B. Moss. 2004. Highly attenuated smallpox vaccine protects mice with and without immune deficiencies against pathogenic vaccinia virus challenge. Proc Natl Acad Sci U S A 101:4590-5.

765. Xiang, Y., R. C. Condit, S. Vijaysri, B. Jacobs, B. R. Williams, and R. H. Silverman. 2002. Blockade of interferon induction and action by the E3L double-stranded RNA binding proteins of vaccinia virus. J Virol 76:5251-9.

766. Xiang, Y., and B. Moss. 1999. IL-18 binding and inhibition of interferon gamma induction by human poxvirus-encoded proteins. Proc Natl Acad Sci U S A 96:11537-42.

767. Xu, R., A. J. Johnson, D. Liggitt, and M. J. Bevan. 2004. Cellular and humoral immunity against vaccinia virus infection of mice. J Immunol 172:6265-71.

768. Xu, X., P. Nash, and G. McFadden. 2000. Myxoma virus expresses a TNF receptor homolog with two distinct functions. Virus Genes 21:97-109.

769. Yang, J., and C. Q. Liu. 2000. Molecular modeling on human CCR5 receptors and complex with CD4 antigens and HIV-1 envelope glycoprotein gp120. Acta Pharmacol Sin 21:29-34.

770. Yang, Y. F., T. Mukai, P. Gao, N. Yamaguchi, S. Ono, H. Iwaki, S. Obika, T. Imanishi, T. Tsujimura, T. Hamaoka, and H. Fujiwara. 2002. A nonpeptide CCR5 antagonist inhibits collagen-induced arthritis by modulating $\mathrm{T}$ cell migration without affecting anti-collagen $\mathrm{T}$ cell responses. Eur J Immunol 32:2124-32.

771. Yip, K. Y., P. Patel, P. M. Kim, D. M. Engelman, D. McDermott, and M. Gerstein. 2008. An integrated system for studying residue coevolution in proteins. Bioinformatics 24:290-2.

772. Yoo, N. K., C. W. Pyo, Y. Kim, B. Y. Ahn, and S. Y. Choi. 2008. Vaccinia virusmediated cell cycle alteration involves inactivation of tumour suppressors associated with Brf1 and TBP. Cell Microbiol 10:583-92. 
773. Yoshimoto, T., K. Takeda, T. Tanaka, K. Ohkusu, S. Kashiwamura, H. Okamura, S. Akira, and K. Nakanishi. 1998. IL-12 up-regulates IL-18 receptor expression on T cells, Th1 cells, and B cells: synergism with IL-18 for IFN-gamma production. J Immunol 161:3400-7.

774. Youssef, S., G. Maor, G. Wildbaum, N. Grabie, A. Gour-Lavie, and N. Karin. 2000. C-C chemokine-encoding DNA vaccines enhance breakdown of tolerance to their gene products and treat ongoing adjuvant arthritis. J Clin Invest 106:361-71.

775. Yu, Q., B. Jones, N. Hu, H. Chang, S. Ahmad, J. Liu, M. Parrington, and M. Ostrowski. 2006. Comparative analysis of tropism between canarypox (ALVAC) and vaccinia viruses reveals a more restricted and preferential tropism of ALVAC for human cells of the monocytic lineage. Vaccine 24:637691.

776. Yu, Y., M. D. Sweeney, O. M. Saad, S. E. Crown, A. R. Hsu, T. M. Handel, and J. A. Leary. 2005. Chemokine-glycosaminoglycan binding: specificity for CCR2 ligand binding to highly sulfated oligosaccharides using FTICR mass spectrometry. J Biol Chem 280:32200-8.

777. Yuwen, H., J. H. Cox, J. W. Yewdell, J. R. Bennink, and B. Moss. 1993. Nuclear localization of a double-stranded RNA-binding protein encoded by the vaccinia virus E3L gene. Virology 195:732-44.

778. Zaitseva, M., K. Peden, and H. Golding. 2003. HIV coreceptors: role of structure, posttranslational modifications, and internalization in viral-cell fusion and as targets for entry inhibitors. Biochim Biophys Acta 1614:51-61.

779. Zeng, F. Y., and J. Wess. 1999. Identification and molecular characterization of m3 muscarinic receptor dimers. J Biol Chem 274:19487-97.

780. Zhang, J., S. S. Ferguson, L. S. Barak, M. J. Aber, B. Giros, R. J. Lefkowitz, and M. G. Caron. 1997. Molecular mechanisms of G protein-coupled receptor signaling: role of $\mathrm{G}$ protein-coupled receptor kinases and arrestins in receptor desensitization and resensitization. Receptors Channels 5:193-9.

781. Zhang, J., E. Rao, M. Dioszegi, R. Kondru, A. DeRosier, E. Chan, S. Schwoerer, N. Cammack, M. Brandt, S. Sankuratri, and C. Ji. 2007. The second extracellular loop of CCR5 contains the dominant epitopes for highly potent anti-human immunodeficiency virus monoclonal antibodies. Antimicrob Agents Chemother 51:1386-97.

782. Zhang, W. H., D. Wilcock, and G. L. Smith. 2000. Vaccinia virus F12L protein is required for actin tail formation, normal plaque size, and virulence. J Virol 74:11654-62.

783. Zhao, J., L. Ma, Y. L. Wu, P. Wang, W. Hu, and G. Pei. 1998. Chemokine receptor CCR5 functionally couples to inhibitory $\mathrm{G}$ proteins and undergoes desensitization. J Cell Biochem 71:36-45.

784. Zhu, J., J. Martinez, X. Huang, and Y. Yang. 2007. Innate immunity against vaccinia virus is mediated by TLR2 and requires TLR-independent production of IFN-beta. Blood 109:619-25. 
785. Zhu, T., H. Mo, N. Wang, D. S. Nam, Y. Cao, R. A. Koup, and D. D. Ho. 1993. Genotypic and phenotypic characterization of HIV-1 patients with primary infection. Science 261:1179-81.

786. Zlotnik, A., and 0. Yoshie. 2000. Chemokines: a new classification system and their role in immunity. Immunity 12:121-7. 